\title{
Synthese und DFT-Studien von Modellkomplexen molybdopterinhaltiger Enzyme
}

\author{
Dissertation
}

zur Erlangung des Doktorgrades

der Mathematisch-Naturwissenschaftlichen Fakultäten

der Georg-August-Universität zu Göttingen

vorgelegt von

Diplom-Chemikerin

Kerstin Starke

aus Lauchhammer

Göttingen 2007 


\section{7}

Referent: Jun.-Prof. Dr. C. Schulzke

Korreferent: Prof. Dr. F. Meyer

Tag der mündlichen Prüfung: 30.10.2007 
Die vorliegende Arbeit wurde unter der Leitung von Jun.-Prof. Dr. Carola Schulzke in der Zeit von Dezember 2003 bis Juni 2007 im Institut für Anorganische Chemie der Georg-August-Universität Göttingen angefertigt.

\section{Frau Jun.-Prof. Dr. Carola Schulzke}

möchte ich an dieser Stelle für die Überlassung des interessanten Themas, ihre hilfreichen Anregungen, ihre Diskussionsbereitschaft und die stete Unterstützung während der Anfertigung dieser Arbeit herzlich danken.

Bei den Damen und Herren der Spektroskopie- und Serviceabteilungen sowie allen weiteren Institutsangehörigen bedanke ich mich für die Hilfsbereitschaft.

Meinen Kolleginnen und Kollegen des Instituts danke ich für das gute Arbeitsklima und die guten Kooperationen. Besonderer Dank gebührt P. M. Gurubasavaraj, U. Nehete, L.W. Pineda, Z. Yang, H. Zhu.

Herzlicher Dank für die gute Arbeitsatmosphäre und hilfreiche Diskussionen gebührt den Mitarbeitern unseres Arbeitskreises A. Döring, M. Heinz, X. Ma und Q. Zhang. 


\section{Abkürzungsverzeichnis}

$\AA$

$\mathrm{AO}$

AOR

Äq.

arom. aromatisch

bdt Benzoldithiolen

ber. berechnet

BO Bindungsordnung

cdt Chromanyldithiolen

delta chemische Verschiebung

DMSO Dimethylsulfoxid

edt Ethylendithiolen

EI Elektronenstoss-Ionisation

FAD Flavinadenindinucleotid

FDH Formiatdehydrogenase

fdt Flavanyldithiolen

FW Faltungswinkel

gef. gefunden 
HMPA Hexamethylphosphoramid

HOMO highest occupied molecular orbital

$\mathrm{Hz} \quad$ Hertz

i.V. im Vakuum

IR Infrarot

J Kopplungskonstante

KLZ Kernladungszahl

LP lone pair, freies Elektronenpaar

LUMO lowest unoccupied molecular orbital

M Molekülion

$\mathrm{m} / \mathrm{e} \quad$ Masse-Ladungsverhältnis

$\mathrm{MHz} \quad$ Megahertz

mnt Maleonitrildithiolen

MO Molekülorbital

mpt Molybdopterin

NAO natürliches Atomorbital

NBO natürliches Bindungsorbital

NMR Kernmagnetresonanz

NPA Natural Population Analysis 


$\begin{array}{ll}\text { NR } & \text { Nitratreduktase } \\ \nu & \text { Frequenz } \\ \text { OS } & \text { Oxidationsstufe } \\ \text { pdt } & \text { Pyranyldithiolen } \\ \text { ppm } & \text { parts per million } \\ \text { prz } & \text { Pyrazindithiolen } \\ \text { RT } & \text { Raumtemperatur } \\ \text { SO } & \text { Sulfitoxidase } \\ \text { TMAOR } & \text { Trimethylamin-N-Oxidoreduktase } \\ \text { TS } & \text { Übergangszustand (transition state) } \\ \text { WBI } & \text { Wiberg Bindungsindex } \\ & \\ & \\ & \end{array}$




\section{Verwendete Liganden}<smiles>SC1=C(S)COCC1</smiles><smiles>SC1=C(S)c2ccccc2OC1</smiles><smiles>SC1=C(S)C(c2ccccc2)Oc2ccccc21</smiles><smiles>SC1=C(S)C2NC=CNC2OC1</smiles><smiles>Nc1nc2c(c(=O)[nH]1)NC1C(S)=C(S)COC1N2</smiles>

$\mathbf{p d t}^{2-} \quad 5,6$-Dihydro-2 $H$-pyran-3,4-dithiolen

$\mathbf{c d t}^{2-} \quad 2 H$-Chromen-3,4-dithiolen

$\mathbf{f d t}^{2-} \quad$ 2-Phenyl-2 $H$-chromen-3,4-dithiolen

prz $^{2-} \quad 4,4 a, 6,8$ a-Tetrahydro- $1 H$-pyrano[2,3- $\left.b\right]$ pyrazin-7,8-dithiolen

mpt $^{2-} \quad$ 2-Amino-3,5,5a,8,9a,10-hexahydro-4H-pyrano[3,2- $g$ ]pteridin-4-on-6,7-dithiolen 


\section{Inhaltsverzeichnis}

Danksagung

Abkürzungsverzeichnis

Verwendete Liganden

$\mathbf{v}$

$1 \quad$ Einleitung

1.1 Der Molvbdopterin-Kofaktor .................. 4

1.2 Wahl und Koordination des Metalls . . . . . . . . . . 8

1.3 Molvbdopterinhaltige Enzvmfamilien . . . . . . . . . . . . . 11

1.3.1 Molvbdän-Hvdroxvlase . . . . . . . . . . . . . . 13

1.3 .2 Sulfit-Oxidase ....................... 14

1.3 .3 DMSO-Reduktase ........................ 15

1.3.4 Aldehvdoxidoreduktase . . . . . . . . . . . . 16

1.4 Stand der Forschung . . . . . . . . . . . . . . . . . 16

1.4.1 Nichtdithiolenverbindungen ................ 18

1.4.2 Svntheserouten zur Dithioleneinheit und deren Übergangsmetallkomplexe 19

1.4 .3 Dithiolenliganden ...................... 21

1.4.4 Svnthese-Ansätze für das Pvranopterin-1,2-En-Dithiolen . . . . 26

1.5 Aufgabenstellung . . . . . . . . . . . . . . . . 28 
2 Ergebnisse und Diskussion $\quad 30$

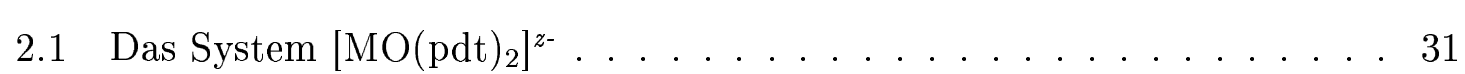

2.1.1 Svnthese von $\left[\mathrm{PPh}_{4}\right]_{2}\left[\mathrm{MoO}(\mathrm{pdt})_{2}\right] \ldots \ldots \ldots . \ldots . \ldots 31$

2.1.2 Geometrische Strukturen . . . . . . . . . . . . . . 34

2.1.3 Elektronische Strukur und Bindung . . . . . . . . . . . . 40

2.2 Die Svsteme $\left[\mathrm{MO}(\mathrm{cdt})_{2}\right]^{z-}$ und $\left[\mathrm{MO}(\mathrm{fdt})_{2}\right]^{z-} \ldots \ldots \ldots \ldots$

2.2.1 Svnthese der Modellkomplexe $\left.\mathrm{PPh}_{4}\left[\mathrm{MO}(\mathrm{cdt})_{2}\right]^{z-}(\mathrm{M}=\mathrm{Mo}, \mathrm{W})\right] \quad 57$

2.2.2 Geometrische Strukturen . . . . . . . . . . . . . 61

2.2.3 Elektronische Struktur und Bindung . . . . . . . . . . 70

2.3 Die Svsteme $\left[\mathrm{MO}(\mathrm{prz})_{2}\right]^{z-}$ und $\left[\mathrm{MO}(\mathrm{mpt})_{2}\right]^{z-} \ldots \ldots . \ldots . \ldots 1$

2.3.1 Ansatz zur Svnthese eines trizvklischen molvbdopterinartigen Liganden 81

2.3.2 Geometrische Strukturen . . . . . . . . . . . . . 83

2.3.3 Elektronische Struktur und Bindung . . . . . . . . . . 89

2.4 Ringöffnung . . . . . . . . . . . . . . . . . . . . 98

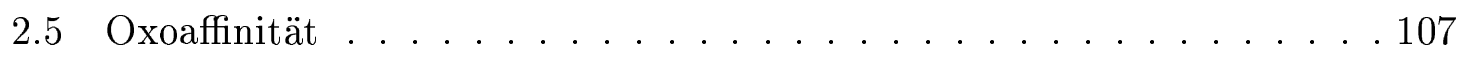

2.6 Diskussion aller Svsteme im Vergleich . . . . . . . . . . . . . . . . 113

2.7 Zusammenfassung und Ausblick . . . . . . . . . . . . . 126

2.8 Summary . . . . . . . . . . . . . . . . . . . . 130

$3 \quad$ Experimenteller Teil $\quad 135$

3.1 DFT-Rechnungen . . . . . . . . . . . . . . . . 135

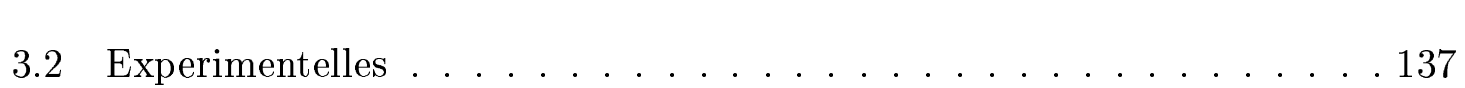

3.3 Darstellung der Ausgansverbindungen . . . . . . . . . . . . . . . 138

3.3.1 Darstellung von 3-Bromo-2.3-dihvdro-4 H-chroman-4-on . . . 138

3.3.2 O-Ethvl-S-(4-oxo-3.4-dihvdro-2 H-chromanon-3-yl)dithiocarbonat 139

3.3.3 $4 H$-[1.3]Dithiolo[4.5-c]chroman-2-on] . . . . . . . . . . . . 140

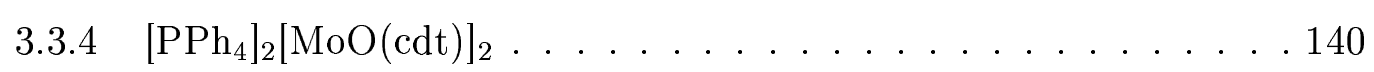




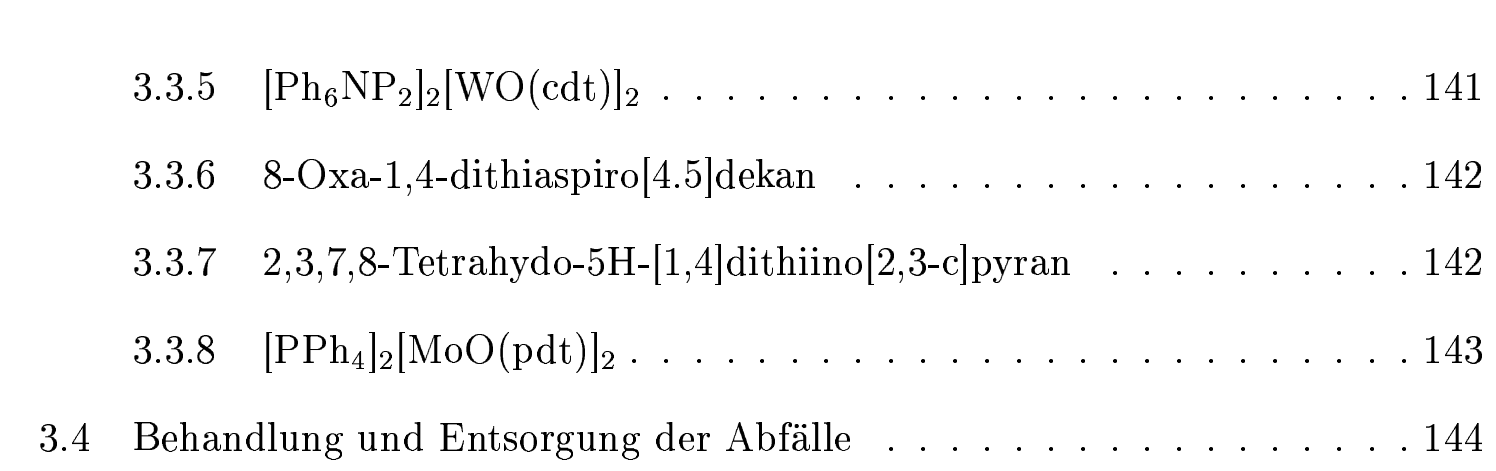

Literaturverzeichnis 


\section{Kapitel 1}

\section{Einleitung}

Molybdän und Wolfram sind die einzigen bekannten Metalle der zweiten (4d) und dritten (5d) Übergangsmetallreihe die als aktive Zentren von Enzymen eine wichtige Rolle für den Metabolismus von Enzymen besitzen. Die Bedeutung von Molybdän und Wolfram ist hinsichtlich ihrer biologischen Funktion seit mehr als 75 Jahren [1] bekannt und seit mehr als 25 Jahren Gegenstand ausführlicher Forschungsarbeiten [2, 3].

Als aktive Zentren in mehr als 50 meist redoxaktiven Metalloenzymen [4] sind sie ubiquitär in Mikroorganismen, Pflanzen und Tieren in der Biosphäre verbreitet. $\mathrm{Zu}$ den enzymatischen Funktionen gehören zahlreiche Schlüsselschritte innerhalb der Stickstoff-, Kohlenstoff-, und Schwefel-Kreisläufe und zwar in Form von SauerstoffÜbertragungs-Prozessen oder der Stickstoff-Fixierung bei Pflanzen.

\subsection{Der Molybdopterin-Kofaktor}

Mit Ausnahme der Nitrogenase [5], welche als mehrkerniges Fe-Mo-S-Zentrum vorliegt, sind die Metalle in allen anderen Enzymen als einkernige Zentren mit einem oder zwei organischen Pterin-Derivaten verknüpft. Die als Oxotransferase klassifizier- 
Abbildung 1.1: Struktur von Molybdopterin<smiles>Nc1nc2c(c(=O)[nH]1)NC1C(S)=C(S)[C@@H](C[18OH])OC1N2</smiles>

ten Enzyme, von denen einige auch als Hydroxylasen eingeordnet werden können, weisen als gemeinsamen Kofaktor den in Abbildung 1.1 gezeigten Pyranopterin-Liganden auf.

Der in Hinblick auf die spätere Entdeckung von Wolfram als Metallzentrum ursprünglich als Molybdopterin (MPT) bezeichnete Kofaktor besteht lediglich aus der organischen Komponente: einem reduzierten Pterin, geknüpft an einen Pyranring, welcher eine Dithioleneinheit beinhaltet, die als zweizähnige Bindungsstelle zum Metall dient. Der Pyranring wird von einer an das Pteringerüst gebundenen phosphorylierten Dihydroxyseitenkette gebildet.

Aufbauend auf den Arbeiten von Patemann et al. [6] und Nason et al. [7, 8], welche als erste das Konzept eines Molybdän-Kofaktors entwickelten, gelang es Rajagopalan und Mitarbeitern, dessen Struktur aufzuklären [9, 10, 11]. Das von ihnen vermutete Gerüst eines Pterins, welches über zwei Schwefelatome an das Metall bindet, konnte durch später folgende Ergebnisse aus Proteinkristallstrukturen bestätigt werden [12, 13, 14, 15, 16].

Dabei stellte sich auch heraus, dass das Pyranopterin sowohl bei molybdän- als auch bei wolframhaltigen Enzymen immer in derselben Form vorliegt und die Bindung des Liganden an das Metall in allen Fällen über die Dithioleneinheit erfolgt. Das Pyranopterin liegt hinsichtlich des Pyran- und Pyrazinrings in einer nichtplanaren Form vor. Die beiden Einheiten bilden einen Winkel von etwa $40^{\circ}$, wobei sich der Pyrazinring an den Atomen C6 exo und C7 endo verdreht. Die chiralen Zentren C6, 
Abbildung 1.2: Öffnung des Pyranrings<smiles></smiles>

C7 und C3' liegen in der $R$-Konformation vor. Der Pyranring nimmt mit dem endo O3' eine Halbsesselkonformation ein.

Diese Konformation liegt nicht starr vor sondern besitzt eine gewisse Flexibilität zwischen den benachbarten Ringen, wie von D. C. Rees et al. [17] anhand einer least-squares-Überlagerung des Pterins beobachtet wurde. Weiterhin ist eine Flexibilität auch aufgrund der Phosphatseitenkette, über die das Molekül mit Nukleotiden verknüpft sein kann, möglich.

Die lange diskutierte Möglichkeit einer Ringöffnung und damit die gegenseitige Umwandlung der typisch trizyklischen in eine bizyklische Form der Pyranopterinstruktur konnte von Bertero et al. [18] durch Strukturdaten unterstützt (Abbildung 1.2).

Das in der kristallisierten NARG-Einheit von E. coli gefundene bicyclische Dihydropterin zeigt, dass sowohl die offene als auch die geschlossene Form, gebunden an das Metall, vorliegen können. Das deutet darauf hin, dass der Kofaktor über die Möglichkeit der Öffnung und Schließung des Pyranrings und des damit verbundenen Einflusses auf den Protonentransfer unmittelbar in den enzymatischen Mechanismus involviert sein könnte. Das vorzugsweise reduziert vorliegende Tetrahydropteridin besitzt in dieser Form kein stark delokalisiertes $\pi$-System. Nach Öffnung des Pyranrings wäre die Möglichkeit gegeben, durch Ausbildung unterschiedlicher mesomerer Formen des Pteridingerüsts ein konjugiertes $\pi$-Elektronensystem aufzubauen und so den 
Elektronentransfer maßgeblich zu steuern.

Bisher ist der genaue Mechanismus mittels dessen das Pyranopterin am katalytischen Prozess beteiligt ist noch nicht geklärt, jedoch scheint zweifelsfrei festzustehen, dass es aufgrund seiner Funktionalität in den Prozess eingebunden ist. Es wechselwirkt nicht nur mit dem direkt gebundenen Metallzentrum sondern auch über umfangreiche Wasserstoffbrückenbindungen mit dem umgebenden Polypeptid [13, 16, 12, 19, 20].

Einen Hinweis auf eine direkte Beteiligung am Elektronentransfer findet man beispielsweise in der Struktur der Aldehyd-Oxidoreduktase von Huber et al.[21], bei der die Aminogruppe des Pterins über eine Wasserstoffbrücke mit einem Schwefel des zum benachbarten 2Fe2S-Cluster gehörenden Cysteins verbunden ist. Damit könnte ein Weg für die nach Ablauf des Elektrontransfers aus dem Metallzentrum entfernten Elektronen vorgegeben sein.

Von Jones und Inscore et al. wurde im Fall der Xanthin-Oxidase [22] sogar ein Mechanismus für den Elektronenaustritt aus dem System vorgeschlagen. Dieser als "Oxogate"-Hypothese [23] formulierte Mechanismus stützt sich auf die räumliche Orientierung des an das Metall gebundenen Sauerstoff-Atoms bezüglich der Dithiolen-Einheit. Xanthin-Oxidase besitzt als Besonderheit ein Sulfid-Atom am Mo-Zentrum, welches für die Katalyse als Protonen- bzw. Hydrid-Akzeptor essentiell ist. Für die Konformation bedeutet das, dass einerseits die Oxo- oder Sulfido-Gruppe des LMoOS-Zentrums cis zur Dithioleneinheit (hier L) stehen kann. Vermutlich findet ein Wechsel der Konformationen während der Substratbindung statt. Hinsichtlich des Elektronentransfers ist der Konformationswechsel von außerordentlicher Bedeutung. Die kovalenten Wechselwirkungen zwischen dem redoxaktiven $\mathrm{d}_{x^{2} y^{2}}$-Orbital des Metalls mit den bindenden p-Orbitalen der Dithioleneinheit werden durch die cis-Ständigkeit der Oxo-Gruppe maximiert. Hohe kovalente Anteile in der Mo-S-Bindung ermöglichen einen leichteren Elektronenfluss aus dem Metallzentrum. Die $\sigma$-Natur der Überlappung ist ebenso von 
enormer Bedeutung, zumal im angrenzenden Pterin keine signifikante $\pi$-Konjugation vorliegt.

Damit kommt dem Pyranopterin eine die elektronische Struktur modulierende Rolle zu. Weitere Studien [24] an Modellsystemen zeigen einen deutlichen "elektronischen Puffer"-Effekt, wobei das Hauptaugenmerk dabei auf den Auswirkungen der Dithioleneinheit auf das Oxidationsverhalten des Metalls liegt.

Die oben genannten Phänomene zeigen, dass der Pyranopterin-Kofaktor sowohl die Struktur als auch die Reaktivität des Metallzentrums beeinflusst, wobei die genauen Abläufe und Vorgänge nach wie vor unbestimmt bleiben.

\subsection{Wahl und Koordination des Metalls}

Innerhalb des Enzyms bestehen verschiedene Möglichkeiten der Koordination des Metallzentrums. Am Metall binden einerseits die Schwefelatome der Dithioleneinheit des Molybdopterins, Proteinseitenketten sowie nicht zum Protein gehörende Gruppen wie Sauerstoff- oder Schwefelatome. Die Schwefel-Metall-Bindungslänge beträgt etwa 2.2 bis $2.4 \AA$, die Metall-Sauerstoff-Bindung ist 1.6 bis $1.8 \AA$ lang. Bei der Koordination von zwei Molybdopterin-Liganden steht das Metall über der von den vier Schwefelatomen gebildeten Ebene und ist quadratisch pyramidal koordiniert. Das Verhältnis von Metall zu Molybdopterin ist beim Molybdän entweder 1:1 oder 1:2, beim Wolfram jedoch immer 1:2. In einigen Proteinen kommen Peptid- oder Aminosäureliganden wie Serinat, Cysteinat oder Selenocysteinat hinzu. Die weiteren an das Metall gebundenen Sauerstoff- oder Schwefel-Atome können als Oxo-, Hydroxo-, Wasser-, Sulfidound Hydrosulfidoliganden vorliegen. In Enzymen aus prokaryotischen Quellen ist das Molybdopterin-System über die Seitenkette gewöhnlich mit Nukleotiden wie Guanin, Cytosin, Adenin oder Hypoxanthin verbunden. Die Anzahl und Art der Liganden 
hängt wiederum von der Oxidationszahl des Metalls ab. Die bisher an Enzymen beobachteten Oxidationszahlen des Metalls bewegen sich zwischen M (VI) und M (IV).

Über die Gründe für die Wahl des Metalls, Molybdän vs. Wolfram, gibt es verschiedene Vermutungen.

Während die Verfügbarkeit von Molybdän und Wolfram für biologisch essentielle Elemente in der Erdkruste sehr gering ist, liegen die Metalle im Meereswasser der Ozeane in recht hohen Konzentrationen vorl. Innerhalb des leicht alkalischen (pH 8.3) und mit Sauerstoff angereicherten Meereswassers liegt Molybdän als dianionisches Molybdat, $\mathrm{MoO}_{4}{ }^{2-}$ vor. In dieser Form ähnelt es strukturell dem Sulfat, welches von Lebewesen assimiliert wird. Obwohl die Verfügbarkeit von Wolfram weitaus knapper ist als die des Molybdäns, ist es an einigen Orten wie z.B. heißen Quelle oder in Salzseen stark angereichert [25].

Ein wesentlicher Unterschied zwischen Molybdän und Wolfram kann an ihrem Verhalten unter anearoben und aeroben Bedingungen festgestellt werden. Unter aeroben Bedingungen liegen die Metalle vorwiegend als Molybdat bzw. Wolframat vor und konkurrieren miteinander um Bindungsstellen und Aufnahmesysteme. Unter anaeroben Bedingungen, wie z. B. in mariner Umgebung, speziell unter hohen Schwefel/SulfidKonzentrationen, wird Molybdän zu Mo(V) oder Mo(VI) reduziert und vorwiegend als $\mathrm{MoS}_{2}$ gefällt. Das Vorherrschen solcher Bedingungen vor allem in der Nähe von heißen Quellen in der Tiefsee führt damit zu einer Verknappung an Molybdän und einer verhältnismäßig hohen Anreicherung von Wolfram.

Wie oben erwähnt, wurde die Bedeutung von Molybdän für biologische Systeme sehr früh erkannt. Molybdoenzyme sind weitverbreitet und wurden bei Bakterien, Archaea und höheren Organismen ausführlich erforscht. Die Bedeutsamkeit von Wolfram innerhalb von biologischen Systemen wurde erst viel später erkannt. Ursprünglich

\footnotetext{
${ }^{1}$ Anteil Erdkruste Molybdän 0.0015\%, Wolfram 0.001\%, Konzentration Meerwasser: Molybdän $10 \mu \mathrm{g} / \mathrm{L}$, Wolfram $0.1 \mu \mathrm{g} / \mathrm{L}$
} 
galt Wolfram als Antagonist des Molybdäns was den Einfluss auf biologische Vorgänge anbelangt. Frühe Versuche, Molybdän in den aktiven Zentren der Molybdoenzyme durch Wolfram zu ersetzen, führten entweder zu inaktiven metallfreien Enzymen oder zu Enzymen mit wenig oder gar keiner Aktivität [26]. Die chemischen Unterschiede zwischen den beiden Metallen sind groß genug, so dass sie innerhalb von Organismen nicht beliebig ausgetauscht werden können. Erst in den 1970ern stieg das Interesse an Wolfram, als ein Einfluss von Wolfram auf das Wachstum von Mikroorganismen beobachtet wurde [27]. Das erste Enzym, welches Wolfram beinhaltete, wurde 1983 aus einem Bakterium isoliert und charakterisiert [28]. Bisher sind etwas mehr als ein Dutzend Wolframenzyme gefunden worden, die ausschließlich aus thermo- und hyperthermophilen Bakterien und Archaea/Mikroorganismen stammen. Die neben heißen und schwefelhaltigen Quellen lebenden Organismen benötigen für ihr Wachstum Temperaturen von etwa $100^{\circ}$. Interessanterweise wurden auch Organismen gefunden, welche beide Metalle verwenden können [29, 30, 31, 32]. Diese bevorzugen allerdings Temperaturen von $50-70^{\circ} \mathrm{C}$. Die hyperthermophilen Organismen $\left(80-130^{\circ} \mathrm{C}\right)$ dagegen bauen ausschließlich Wolfram in ihre aktiven Zentren ein.

Die Betrachtung der geologischen und biologischen Verfügbarkeit der Metalle sowie ihr unterschiedliches chemisches Verhalten vor allem bei unterschiedlichen Temperaturen führt zu verschiedenen Vermutungen hinsichtlich der Wahl des Metalls. Diskutiert werden einerseits ein zielgerichteter und andererseits ein angebotsbestimmter Einbau.

Die erwähnten unterschiedlichen Bedingungen der verschiedenen Lebensräume scheinen einen Einfluss auf die für den katalytischen Zyklus notwendigen Redoxpotentiale zu haben. Somit könnten die unterschiedlichen Redoxpotentiale der Metalle ein Grund dafür sein, dass das schwerer zu reduzierende Wolfram in die bei niedrigerem Redoxpotential arbeitenden Wolfram-Enzyme eingebaut wird. Darüberhinaus besitzen Wolframkomplexe wesentlich stabilere Wolfram-Schwefel-Bindungen, welche vor 
allem bei Bedingungen mit hohen Temperaturen einen Vorteil gegenüber Molybdän darstellen.

Andererseits können die chemischen Unterschiede hinsichtlich der geochemischen Entwicklung dazu beigetragen haben, dass sich durch die unterschiedliche Verfügbarkeit Präferenzen zum Einbau eines bestimmten Metalls entwickelten. Wie oben erwähnt, kommt Molybdän zwar unter Normalbedingungen häufiger vor, liegt aber in den Lebensräumen der Wolframenzyme aufgrund hoher Sulfidkonzentrationen nicht in assimilierbarer Form vor, sondern ist als $\mathrm{MoS}_{2}$ gefällt. Wolfram hingegen tritt unter diesen Bedingungen als $\mathrm{WO}_{2} \mathrm{~S}_{2}{ }^{2-}$ oder als $\mathrm{WOS}_{3}{ }^{2-}$ auf und wird vermutlich auch in dieser Form in die Organismen eingebaut.

Die endgültige Aussage darüber, ob die extremen Bedingungen wie niedrige Potentiale und hohe Temperaturen Wolfram erfordern oder das natürliche Vorkommen beider Metalle die Auswahl von Molybdän oder Wolfram bestimmen ist noch nicht geklärt und bedarf weitergehender Untersuchungen.

\subsection{Molybdopterinhaltige Enzymfamilien}

Enzyme, die Molybdän und Wolfram in ihre aktiven Zentren einbauen sind in allen Lebensformen, von Archaea bis zu Säugetieren allgegenwärtig. Bei den von ihnen katalysierten Reaktionen innerhalb der C-, S-, und N-Kreisläufe wird Sauerstoff in Form von $\mathrm{O}^{2-}$ von Wasser auf das Substrat bzw. vom Substrat auf Wasser übertragen, beschrieben durch die generelle katalytische Reaktion [33]

(1) $\mathrm{X}+\mathrm{H}_{2} \mathrm{O} \rightleftharpoons \mathrm{XO}+2 \mathrm{H}^{+}+2 \mathrm{e}^{-}$

Molybdopterinhaltige Enzyme können anhand ihrer Aminosäuresequenzen, spektroskopischer Eigenschaften, den Strukturen der aktiven Zentren sowie den von ihnen katalysierten Reaktionen klassifiziert werden. 
Abbildung 1.3: Schema der molybdopterinhaltigen Enzymfamilien in OS VI
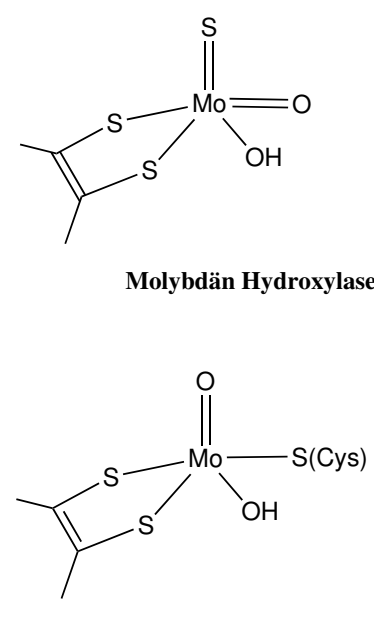

Sulfit Oxidase
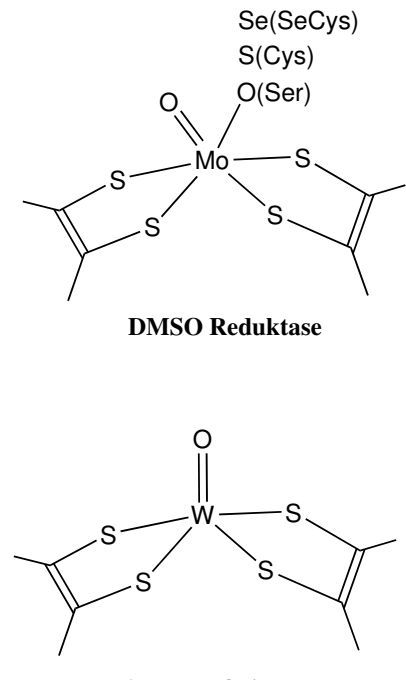

Aldehyd Oxidoreduktase

Bei der Unterteilung hinsichtlich ihrer Funktion unterscheidet man Oxotransferasen und Hydroxylasen. Von Oxotransferasen ist die Rede, wenn das Substrat in einen direkten Sauerstoff-Atom-Transfer involviert ist, Hydroxylasen dagegen katalysieren Hydroxylierungen und bevorzugen Wasser als Sauerstoffquelle [34].

Nach der Klassifizierung von R. Hille werden molybdänhaltige Enzyme in drei verschiedenen Klassen unterteilt, dazu kommt noch eine weitere Klasse der wolframhaltigen Enzyme [35, 36, 37, 4]. Im Einzelnen sind dies die Familien Molybdän Hydroxylase, Sulfit Oxidase und DMSO Reduktase im Fall der molybdänhaltigen Enzyme und die Aldehydoxidoreduktase (AOR) bei den wolframhaltigen Enzymen.

Innerhalb der vergangenen Jahrzehnte wurde eine steigende Zahl von Proteinkristallstrukturen, welche über einkernige Molybdän- bzw. Wolframzentren verfügen, aufgeklärt, so dass in jede Familie und Unterfamilie dieser Enzyme wertvolle Einblicke gewährt werden können. 


\subsubsection{Molybdän-Hydroxylase}

Die Familie der Molybdän-Hydroxylasen zählt mit mehr als 20 charakterisierten Enzymen zur größten Gruppe einkerniger Molybdänenzyme. Diese katalysieren die oxidative Hydroxylierung verschiedenster Aldehyde, aromatischer Heterocyclen und des Kohlenmonoxids [35].

Molybdän Hydroxylasen sind im engeren Sinne Oxidasen, die Wasser als Quelle für den einzubauenden Sauerstoff benutzen. Zu dieser Familie zählen unter anderem die sowohl von höheren Organismen als auch von Bakterien stammenden gut charakterisierten Xanthin- (XO) und Aldehyd-Oxidoreduktasen (AOR) oder auch AldehydOxidase (AO) sowie die Formiat-Dehydrogenase (FDH). Die Aldehyd-Oxidoreduktase von Desulfovibrio gigas war beispielsweise das erste durch Röntgenkristallstrukturanalyse aufgeklärte mononukleare Molybdänenzym [38].

Wie in Abbildung 1.3 gezeigt, besteht das aktive Zentrum dieser Enzymgruppe aus einer LMo(VI)OS(OH)-Einheit an die nur ein Pyranopterin-Kofaktor gebunden ist. Das Metall ist dabei quadratisch-pyramidal koordiniert.

Eine Besonderheit stellt die Koordination am Metallzentrum dar, wobei die Mo-SGruppe eine epikale Stellung einnimmt und die Mo-O-Gruppe in die axiale Position, in trans-Stellung zur Dithiolen-Einheit, verdrängt wird.

Das Molybdän-Zentrum der CO-Dehydrogenase hingegen weist mit einem aktiven Zentrum der Form LMo(VI) $\mathrm{O}_{2}$ einen signifikanten Unterschied hinsichtlich der Struktur auf [39]. Völlig ungewöhnlich ist auch die Anwesenheit eines über ein $\mathrm{Cu}(\mathrm{I})$-Atom mit dem aktiven Zentrum verknüpften Selanylcystein-Rests, dessen Einfluss auf den katalytischen Zyklus bis heute nicht gänzlich geklärt ist.

Allen Enzymen der Hydroxylase-Familie ist gemein, dass sie neben dem Molybdän über weitere redoxaktive Zentren, wie z.B. Fe-S-Cluster, verfügen. Die aus dem Redoxprozess stammenden Elektronen können damit über einen intramolekularen Weg 
zu externen Akzeptoren geleitet werden.

\subsubsection{Sulfit-Oxidase}

Die Sulfit Oxidase Familie umfasst die eukaryotischen Molybdoproteine Sulfit Oxidase (SO), welche ubiquitär in Tieren verbreitet ist und meist aus Hühner- oder Rattenleber isoliert wurde, sowie die Nitrat Reduktase (NR) welche in Algen, Pilzen und höheren Pflanzen vorkommt.

Dabei katalysiert die Sulfit-Oxidase die physiologisch grundlegende Oxidation von Sulfit zu Sulfat, die Nitrat-Reduktase die Reduktion von Nitrat zu Nitrit.

Das aktive Zentrum besteht aus einem fünffach koordiniertem Molybdän-Atom von annähernd quadratisch-pyramidaler Koordination. Die äquatoriale Ebene ist dabei von drei S-Atomen und einem Wasser/Hydroxido-Liganden besetzt; in axialer Stellung befindet sich ein Oxo-Ligand. Zwei der äquatorialen Schwefel-Atome stammen aus der Dithiolen-Einheit des Pyranopterin-Liganden während das dritte Schwefel-Atom aus einem anknüpfendem Cystein stammt. Das Cystein kommt in allen SO und NR vor und ist für diese Gruppe von Enzymen charakteristisch. Die Bedeutung des ThiolatLiganden konnte durch den Austausch mit Serinat, welcher zu inaktiven Enzymen führte, nachgewisen werden [40].

Wie auch bei den Hydroxylasen befinden sich in der SO und in der NR zusätzliche redoxaktive Zentren neben dem Molybdän, z.B. ein B-typ Cytochrom bei der SR [41] und der NR, neben welchem sich auch noch eine FAD-Einheit befindet [42]. XAS-Messungen der oxidierten und reduzierten Form einer der NR-Vertreter wiesen Änderungen der Mo-S-Bindungen im Verlauf des Redoxprozesses nach und zeigen somit einen deutlichen Einfluss des katalytischen Zyklus auf die Struktur des aktiven Zentrums [43]. 


\subsubsection{DMSO-Reduktase}

Enzyme der DMSO Reduktase Familie katalysieren einen eigentlichen Sauerstofftransfer zu oder von einem erreichbaren Elektronenpaar am Substrat. Typische Vertreter dieser Familie sind Enzyme aus Bakterien und Archaea wie die DMSO Reduktase (DMSOR), Biotin-S-Oxid-Reduktase, Trimethylamin Oxidoreduktase (TMAOR), Formiatdehydrogenase (FDH), Arsenit-Oxidase (AO) und viele mehr. Die strukturell am vielfältigsten aufgestellte Familie der Molydoenzyme besitzt jeweils zwei Pyranopterin-Kofaktoren pro Metall-Atom, an das noch zwei weitere Liganden gebunden sind und welches in trigonal-prismatischer Koordination vorliegt. Als weitere Liganden wurden neben einer Oxo-Gruppe Cystein, Aspartat, Serin (NR von Desulfovibrio desulfuricans [44]), Selenocystein (Escherichia Coli FDH [45]) oder aber eine Hydroxygruppe (AOR Alcaligenes faecalis[46]) beobachtet. Bei den Fällen, in denen das Metall an eine Aminosäure bindet, ist die Mo-Oxo-Bindung länger als gewöhnlich und erleichtert damit eine Protonierung zu dem Fragment Mo-OH. Die Mo-O Bindung des aminosäurefreien aktiven Zentrums dagegen entspricht hinsichtlich der Bindungslänge eher einer Mo-O-Doppelbindung. Innerhalb der DMSO-R-Familie liegen nicht nur vielfältige Strukturen vor, sondern auch Anzahl und Organisation der weiteren redoxaktiven Zentren ist recht vielfältig. Enzyme wie die in Rhodobacter vorkommende DMSO-Reduktase, TMAO-Reduktase oder die Biotin-S-Oxid-Reduktase besitzen neben dem Molybdän keine weiteren redoxaktiven Zentren. Die Nitratreduktase, Formiatdehydrogenase und die Arsenit Oxidoreduktase hingegen verfügen über weitere Fe-S-Zentren innerhalb desselben Polypeptids. Die Arsenit Oxidoreduktase verfügt sogar über eine weitere Untereinheit, die ein Rieske-Typ 2Fe/2S Zentrum beinhaltet. 


\subsubsection{Aldehydoxidoreduktase}

Die Wolfram beinhaltenden Enzyme sind der Aldehydoxidoreduktase Familie zugeordnet. Derzeit sind 14 Enzyme von anaereoben, meist thermophilen Organismen charakterisiert worden. Diese Enzyme sind wiederum in drei Klassen unterteilt worden: die Aldehydreduktase (AOR), die Formiatdehydrogenase (FDH) und die Acetylen Hydratase [47]. Während für Vertreter aus der AOR sowie der Acetylen Hydratase [48] Kristallstrukturen bestimmt worden sind, gibt es für Vertreter der Formiatdehydrogenase bisher noch keine aufgeklärten Strukturen.

Die meisten Wolfram-Enzyme gehören der AOR Klasse an und katalysieren die Oxidation von Aldehyden zu den entsprechenden Carbonsäuren wobei Ferredoxin als physiologischer Elektronen-Akzeptor dient. Neben dem Wolfram als redoxaktivem aktivem Zentrum liegen auch hier verschiedene weitere redoxaktive Zentren vor wie z.B. $4 \mathrm{Fe}-4 \mathrm{~S}-\mathrm{Cluster}$.

Die strukturellen Daten der AOR von P. furiosus [49] zeigten, dass das Wolfram von zwei Pyranopterinliganden, also von vier Schwefelatomen verzerrt quadratisch pyramidal umgeben ist. An das Metall koordinierende Proteinreste, wie bei anderen Familien gefunden, wurden nicht beobachtet. Eine verbliebene Restelektronendichte deutet auf eine Oxo-Gruppe. Die Einbindung der beiden Pyranopterinliganden innerhalb des Enzyms erfolgt hier nur über die Phosphatgruppen der Seitenkette des Pyranrings.

\subsection{Stand der Forschung}

Vereinfachte Modelle von komplexen Strukturen sollen den Zweck erfüllen, komplexe Mechanismen und Funktionen leichter aufklären und verstehen zu können. Nicht nur die vielfältigen Strukturen der aktiven Zentren innerhalb der Molybdän- und Wolfram- 
komplexe, sowohl die Koordination der Metalle als auch der Aufbau und die Funktion des Pyranopterinliganden, sondern auch ihre lebensnotwendige Bedeutung und ihre Vielseitigkeit gibt Anlass zu immer weitergehenden Forschungen.

Mit der Absicht aus synthetisierten vereinfachten Modellverbindungen Informationen über chemische und physikalische Eigenschaften sowie den Ablauf der katalytischen Zyklen zu gewinnen, steigerte sich die Anzahl der Syntheserouten drastisch. Hinsichtlich der Natur der aktiven Zentren und des katalytischen Zyklus' wurden zahlreiche Forschungsarbeiten bezüglich des molybdänbezogenenen Sauerstofftransfers durchgeführt [50]. Hierbei lag das Hauptaugenmerk primär auf der Sauerstofftransferreaktion (2) bei der das Sauerstoffatom durch direkten Angriff des Substrats auf das Metallzentrum übertragen wird.

(2) $\mathrm{Mo}^{\mathrm{IV}} \mathrm{O}+\mathrm{XO} \rightleftharpoons \mathrm{Mo}^{\mathrm{VI}} \mathrm{O}_{2}+\mathrm{X}$

(3) $\mathrm{Mo}^{\mathrm{IV}} \mathrm{O}+\mathrm{Mo}^{\mathrm{VI}} \mathrm{O}_{2} \rightleftharpoons \mathrm{Mo}_{2} \mathrm{O}_{3}$

Nach Aufklärung verschiedenster Strukturen der aktiven Zentren ergaben sich neue Herausforderungen in der Synthesechemie. Während $\mathrm{Mo}^{\mathrm{IV}} \mathrm{O} / \mathrm{Mo}^{\mathrm{VI}} \mathrm{O}_{2}$ häufig in der konventionellen Molybdänchemie anzutreffen sind, musste nun auch das anspruchsvollere Paar $\mathrm{Mo}^{\mathrm{IV}} / \mathrm{M}^{\mathrm{VI}} \mathrm{O}$ für Untersuchungen herangezogen werden. Weitere koordinative Fragestellungen beinhalteten die Formation der ungewöhnlichen $\mathrm{Mo}^{\mathrm{VI}} \mathrm{OS}-\mathrm{Gruppe}$, die Stabilisierung des fünffach-koordinierten $\mathrm{Mo}^{\mathrm{VI}} \mathrm{O}_{2}$-Zentrums unter Einfluss der stark reduzierend wirkenden Thiolat-Liganden sowie die Synthese von Mono- und Desoxozentren mit adäquaten Ligandensystemen. Aufgrund der Neigung zur Dimerisierung (3) des Molybdäns galt es sowohl, Ligandensysteme mit sterischem Anspruch zu entwickeln, die diese unerwünschte Nebenreaktion ausschlossen, als auch das Pyranopterin adäquat zu modellieren.

Hinsichtlich dieser Problematik entwickelte sich die analoge Wolframchemie wesentlich später und nicht in dem Maße. Erst mit der Entdeckung der wolframhaltigen 
Enzyme erreichte dieses Gebiet eine steigende Aufmerksamkeit. So erlangte die relevante Dithiolen- sowie Oxotransferchemie von Wolfram erst in den 90ern [50, 51] allgemeines Interesse. Die isostrukturellen und isoelektronischen Wolframkomplexe wurden hinsichtlich ihres Reaktionsverhaltens, kinetisch und elektrochemisch analog zu ihren Molybdänverbindungen untersucht [52, 53, 54, 55, 56]. Der Sauerstoff-TransferReaktion mit Wolfram jedoch kommt zu Gute, dass die unerwünschte Dimerisierung hier ausbleibt.

Abgesehen von der Koordination des Metalls ist es nach wie vor eine große Herausforderung dem synthetisch anspruchsvollen Pyranopterin gerecht zu werden. Der Ligand beinhaltet mit seinem an einen dithiolentragenden Pyranring geknüpften Pteridin eine sehr ungewöhnliche Kombination von insgesamt drei redoxaktiven Zentren. Hinzu kommt eine extreme Instabilität des Kofaktors, was sich auch daran zeigt, dass bisher keiner in völlig intaktem Zustand isoliert und strukturell charakterisiert werden konnte [57, 58]. Neben der leichten Dissoziation des Metalls ist das reduzierte Pterin gegenüber Sauerstoff sehr empfindlich, was selbst bei der Strukturaufklärung im Protein ein großes Problem darstellt.

\subsubsection{Nichtdithiolenverbindungen}

Das Metallzentrum der SO beispielsweise ist fünffach koordiniert und mit drei ThiolatDonoren verknüpft, eines vom Cystein und zwei vom Dithiolenliganden stammend. Dazu kommen zwei Oxo-Gruppen bei Erreichen der vollständigen Oxidation [59, 60]. Eine derartige Koordination ist für das Metall sehr ungewöhnlich. Die Synthese solcher Modelle ist weiterhin durch die Komproportionierungsneigung (3) der Molybdänverbindungen in Hinblick auf den Sauerstofftransfer beeinträchtigt. Hauptaugenmerk liegt daher auf der Entwicklung sterisch anspruchsvoller Liganden die das Metallzentrum derart abschirmen, dass das Gleichgewicht von (3) weit auf der linken Seite liegt. 
Abbildung 1.4: Systeme mit sterisch hindernden Liganden
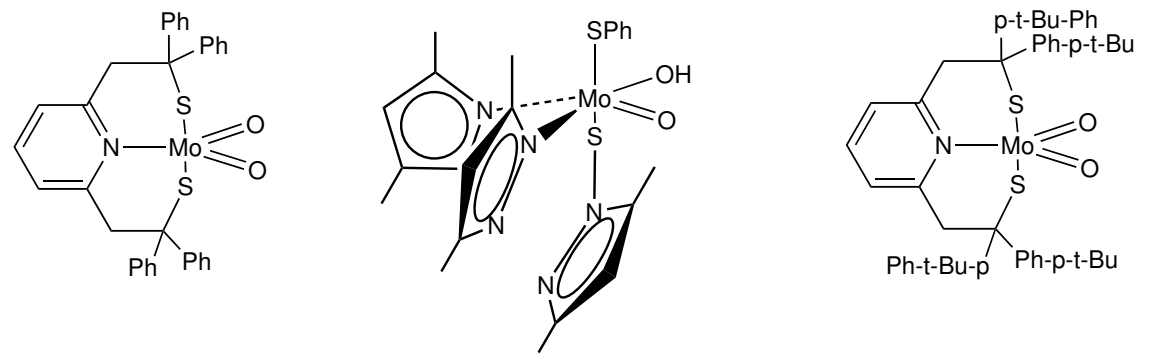

Eine der ersten Verbindungen, die diese Voraussetzungen erfüllten, wurde von Berg und Holm [61, 62] entwickelt.

Diese Systeme der zweiten Generation beinhalten sterisch anspruchsvolle Liganden (Abbildung 1.4), die über S und N koordinieren [63, 64].

Anhand dieser Systeme konnte der Oxotransfer für zahlreiche Substrate X/XO untersucht werden, darunter $\mathrm{Et}_{3} \mathrm{P}$, S-oxide, N-Oxide und $\mathrm{Ph}_{2} \mathrm{SeO}$. Aufgrund der erhaltenen kinetischen Daten konnte gezeigt werden, dass die Sauerstoff-Übertragung einem Geschwindigkeitsgesetz zweiter Ordnung mit einem assoziativen Übergangszustand folgt. Die Fortschritte dieser Untersuchungen gaben auch Anlass zu theoretischen Analysen [65, 66] welche die angenommene Kinetik untermauerten. Eine weitere Entwicklung ergab sich durch den Einsatz des 3,5-disubstituierten Trispyrazolyborats $\left(\mathrm{Tp}^{*}\right)$ als Liganden mit Verbindungen des Typs $\left[(\mathrm{Tp}) * \mathrm{Mo}^{\mathrm{VI}} \mathrm{O}_{2} \mathrm{X}\right](\mathrm{X}=\mathrm{Cl}, \mathrm{Br}, \mathrm{OPh})$.

Das Strukturelement $\left[(\mathrm{Tp})^{*} \mathrm{MoO}(\mathrm{X})_{2}\right]$ bietet eine große Anzahl an stabilen $\mathrm{Mo}^{\mathrm{V}} \mathrm{O}$ Komplexen [67, 68] die mit Hilfe von EPR-Techniken untersucht werden konnte.

\subsubsection{Syntheserouten zur Dithioleneinheit und deren Über- gangsmetallkomplexe}

Die Koordination des Pyranopterinliganden an das Metall in der Natur erfolgt, wie in kristallographischen und spektroskopischen Studien gezeigt [69, 70], über Schwefel- 
Abbildung 1.5: Klassifizierung der Dithiolene nach Holm

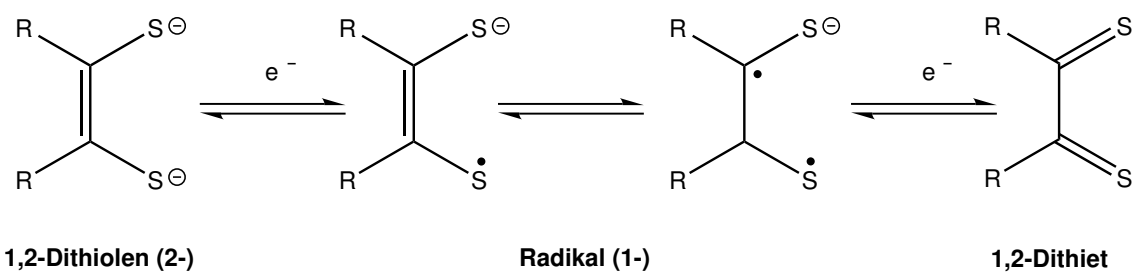

Atome welche einer Dithioleneinheit angehören.

Dithiolene mit der allgemeinen Form $\mathrm{R}_{2} \mathrm{C}_{2} \mathrm{~S}_{2}{ }^{2-}$ sind sogenannte non-innocentLiganden. Sie können in Abhängigkeit von ihrer Oxidationsstufe verschiedene Formen zwischen den Extremen des neutralen Dithiets und des dianionischen Dithiolats annehmen (Abbildung 1.5). Diese Fähigkeit bedingt ihr ungewöhnliches vielfältiges chemisches Verhalten.

Die ersten eingehenderen Forschungsarbeiten an Dithiolenen begannen in den frühen 1960ern aufgrund eines Interesses an dem ungewöhnlichen Redoxverhalten und den strukturellen Besonderheiten dieser Verbindungen [71, 72, 73, 74]. Neben der Grundlagenforschung entwickelte sich kurz darauf auch ein materialwissenschaftliches Interesse an diesen Verbindungen. Die besondere Eignung solcher redoxaktiven Verbindungen prädestiniert sie für Anwendungen in elektronischen Bereichen, z.B. als elektronische Leiter oder Schalter [75]. Die präparative Chemie der Dithiolene partizipierte darüber hinaus auch an Fortschritten und Erkenntnissen der Tetrathiofulvalenchemie [76]. Letztendlich kam in den 1990ern aufgrund der biologischen Entdeckung der Molybdän- und Wolframenzyme ein erneutes Interesse zur Erweiterung und Verbesserung von Syntheserouten hinzu. 
Abbildung 1.6: Strukturen der anorganischen Dithiolene<smiles>S=c1sc([Se])c([Se])s1</smiles>

dmit<smiles>N#CC([SeH3])=C([SeH3])[SeH3]</smiles>

mnt<smiles>C=C[Os]</smiles>

edt<smiles>O=S(=O)([O-])c1ccccc1S</smiles>

bdt

\subsubsection{Dithiolenliganden}

Einfachste Vertreter, die genügen, um eine Koordination der Dithioleneinheit zu erreichen sind neben dem Benzoldithiol die anorganischen Dithiolene 1,3-Dithiol-2-thion4,5-dithiolat (dmit $\left.{ }^{2-}\right)$, 1,2-Maleonitril-1,2-dithiolat (mnt ${ }^{2-}$ ) oder das 1,2-Ethendithiolat $\left(\right.$ edt $\left.^{2-}\right)$ (Abbildung 1.6), die einen einfachen synthetischen Zugang haben.

Die ersten veröffentlichten Strukturen von homoleptischen Bis(dithiolen)komplexen sind $\left[\mathrm{Ni}(\mathrm{mnt})_{2}{ }^{2-}\right]$ von Eisenberg et al. [77, 78] und das $\mathrm{Ni}\left[\mathrm{S}_{2} \mathrm{C}_{2}(\mathrm{Ph})_{2}\right]_{2}$ von Schrauzer und Mayweg [74]. Bis heute sind über 400 Strukturen von dithiolentragenden Übergangsmetallverbindungen bekannt, wobei aufgrund ihrer Anwendungen in den Materialwissenschaften ein großes Interesse an Nickelverbindungen besteht [79].

Aromatische Dithiole wie das Benzoldithiol sowie verschieden substituierte Derivate sind recht stabil und gehören mit einer breiten Verwendung zu den wertvollen Synthesebausteinen. Ihre Darstellung erfolgt in Anlehnung an die klassische Etherspaltung [80] durch reduktive Dealkylierung von Verbindungen des Typs $\mathrm{C}_{6} \mathrm{R}_{4}\left(\mathrm{SR}^{\prime}\right)_{2}$. Diese wiederum sind durch Substitution dihalogenierter Aromaten mittels Alkalimetallen zugänglich. In einer neueren Variante werden Benzolthiole mit BuLi zu 2- $\mathrm{LiC}_{6} \mathrm{H}_{4}(\mathrm{SLi})$ umgesetzt, welches dann mit elementarem Schwefel das gewünschte Dithiolen ergibt (Abbildung 1.7) [81, 82].

Die Umsetzung zum Übergangsmetallkomplex erfolgt typischerweise über Eliminierungen von Metallhalogeniden, Thiolaustausch oder Kondensation des freien Thiols 
Abbildung 1.7: Darstellung von Benzoldithiolen

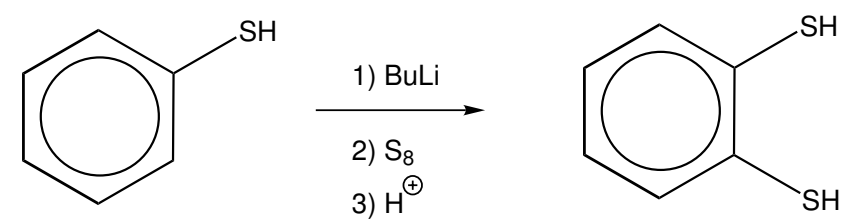

mit Oxo, Alkoxy- oder Amido-Vorstufen und anschließender Oxidation. Im Fall der Reaktionen von $\mathrm{MoCl}_{5}$ und $\mathrm{WCl}_{6}$ besteht jedoch das Problem der Bildung von TrisDithiolenkomplexen $\left[\mathrm{M}\left(\mathrm{S}_{2} \mathrm{C}_{6} \mathrm{R}_{4}\right)_{3}\right]^{\mathrm{z}}(\mathrm{M}=\mathrm{Mo}, \mathrm{W} ; \mathrm{z}=0,-1,-2)$ [83, 84] welche in Hinblick auf die Modellierung von Modellkomplexen für molybdopterinhaltige Enzyme unerwünscht sind. Um Oxo-Bis-Dithiolene $\left[\mathrm{MO}\left(\mathrm{S}_{2} \mathrm{C}_{6} \mathrm{R}_{4}\right)_{2} 2\right]^{\mathrm{z}}(\mathrm{M}=\mathrm{Mo}, \mathrm{W} ; \mathrm{z}=0$, -1, -2] der Metalle zu erhalten sind daher besondere Reaktionsführungen bei der Metallumsetzung erforderlich, um die Bildung von Trisdithiolenkomplexen zu vermeiden.

Der Zugang zu 1,2-Alkendithiolenen ist im Vergleich zu den aromatischen Quasien-dithiolenen durch ihre leichte Zersetzung und den größeren Einfluss ihrer Substituenten auf den Reaktionsablauf wesentlich erschwert. Die zu der oben besprochenen analoge reduktive Dealkylierung führt bis auf wenige Ausnahmen zu instabilen, stark reduzierenden und damit nicht isolierbaren Alkendithiolaten. Ihre Isolierung und Charakterisierung erfolgt gewöhnlich nach einer Transformierung mittels der Umsetzung mit einem Nukleophil, wie z.B. Methyliodid oder einer unmittelbar folgenden Umsetzung mit dem entsprechenden Metall. Die Thioetherspaltung selbst verläuft im Gegensatz zur Etherspaltung wesentlich leichter und ist ihr auch im Hinblick auf die Regioselektivität überlegen [85]. Die stark reduzierenden Bedingungen der dabei verwendeten Reagenzien wie $\mathrm{Na}$ in HMPA erlauben jedoch keine große Auswahl an zusätzlichen funktionellen Gruppen.

Von Garner und Joule [86] wurde mit der basischen Hydrolyse von Dithiocarbonaten ein wertvoller Zugang zu Dithiolenen entwickelt, der aufgrund milderer Bedingun- 
Abbildung 1.8: Syntheseroute nach Garner<smiles>[R]C(=O)C([R9])C(C)=O</smiles>

Abbildung 1.9: Radikalische Spaltung von Diisopropylxanthogendisulfid

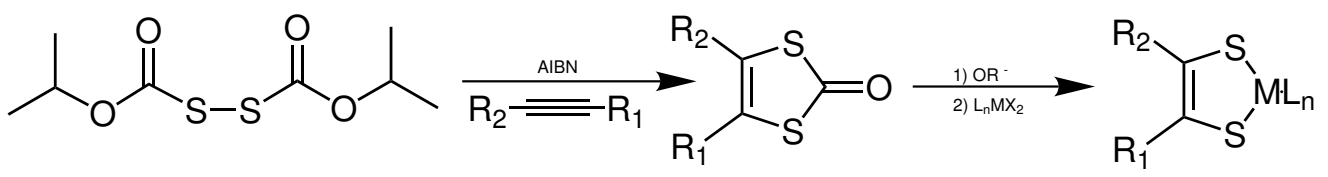

gen eine vielfältigere Auswahl an Substituenten am Rückgrat des Dithiolens zuläßst (Abbildung 1.8). Die Dithiocarbonate können auf verschiedenen Wegen synthetisiert werden. Die durch Substitution von $\alpha$-Bromoketonen erhaltenen $\alpha$-Keto-xanthogenate lagern sich säurekatalysiert unter Wasserabspaltung zu den gewünschten Dithiocarbonaten um.

Diese Umsetzung gelingt ebenso mit den entsprechenden Carbamaten welche zu den Iminium-Analoga führen. Beide Spezies lassen sich unter basischen Bedingungen zum freien Dithiolen spalten, welches in situ mit dem Metall-Precursor zum gewünschten Komplex weiterreagieren kann. Um die Bildung von Tris-Dithiolenen zu vermeiden, muss auch hier auf die Auswahl geeigneter Metallvorstufen geachtet werden.

Eine weitere Möglichkeit Dithiocarbonate zu erhalten, ist die formale Addition der " $\mathrm{CS}_{2} \mathrm{O}$ "-Einheit an eine Dreifachbindung. Unter radikalischen Bedingungen erfolgt die Spaltung von Diisopropylxanthogendisulfid, welches nach Angriff der Dreifachbindung und intramolekularer Umlagerung das Dithiocarbonat ergibt [87].

Auch hier ist der Nachteil, dass die Art der eingeführten funktionellen Gruppe keine große Auswahl zulässt. Um die Dreifachbindung zu aktivieren, ist es notwendig, stark elektronenziehende Substituenten zu verwenden. 
Die Addition an eine C-C-Dreifachbindung führt auch bei der von Stiefel et al. verwendeten Route [88] zum Dithiolenkomplex. Hierbei reagiert die aktivierte C-CDreifachbindung in der Seitenkette eines Quinoxalins mit einem MolybdänpersulfidoKomplex zur gewünschten Metallverbindung. Mittels dieser Reaktion (Abbildung 1.10) konnten Quinoxaline, die eine Dreifachbindung in der Seitenkette tragen, umgesetzt werden und ergaben Modellkomplexe, die den Pteridinteil des Kofaktors imitieren 89$]$.

Abbildung 1.10: Addition an eine Dreifachbindung
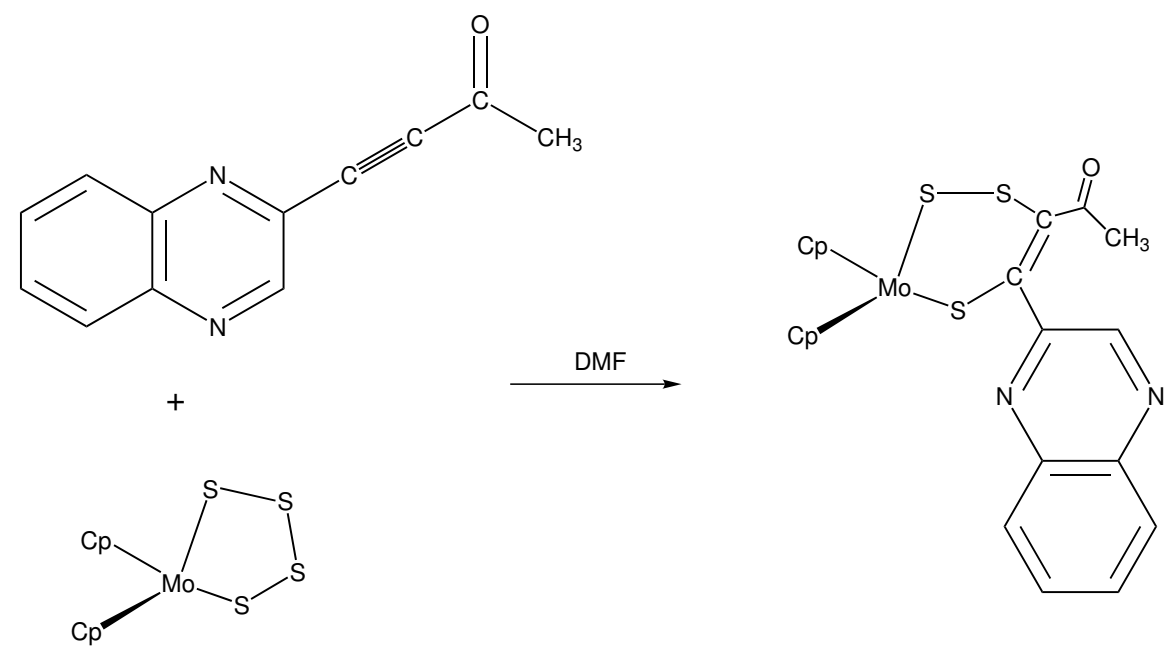

\begin{tabular}{l|l}
$i$ & + \\
$\frac{0}{0}$ \\
$\frac{0}{\bar{v}}$ \\
$\frac{0}{0}$ \\
$\bar{w}$
\end{tabular}<smiles>CC(=O)C1=C(c2cnc3ccccc3n2)SN(C2CCCCC2)S1</smiles>

Die erste auch für Alkyl- und Arylsubstituenten allgemein bewährte Synthese- 
Abbildung 1.11: Syntheseroute nach Schrauzer und Mayweg

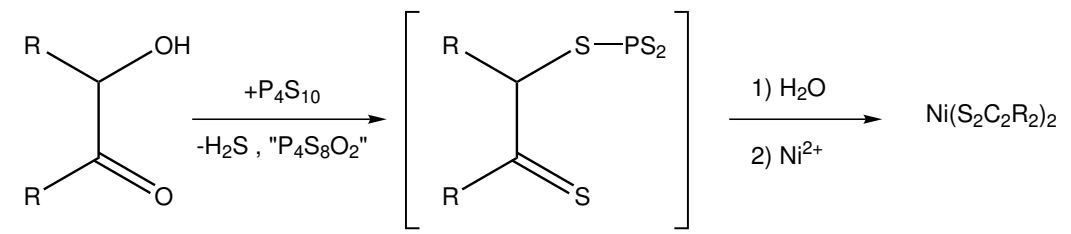

route von Schrauzer und Mayweg [74] führt über einen Thiophosphatester, der aus einem Acyloin ( $\alpha$-Hydroxyketon) durch Umsetzung mit $\mathrm{P}_{4} \mathrm{~S}_{10}$ erhalten wird (Abbildung 1.11).

Das nicht näher spezifizierte $\alpha$-Mercaptothion ergibt durch die direkte Umsetzung mit Metallsalzen die Dithiolenkomplexe. Auf diesem Weg wurden vor allem zahlreiche Nickelkomplexe der Form $\mathrm{Ni}\left(\mathrm{S}_{2} \mathrm{C}_{2} \mathrm{R}_{2}\right)_{2}(\mathrm{R}=\mathrm{Me}$ und $\mathrm{Ph})$ erhalten. Diese sind als Dithiolen-Transfer-Agenzien vor allem für die Synthese von Molybdän- und Wolframkomplexen interessant geworden.

Eine dieser Route ähnelnde Methode ist die Umsetzung von $\alpha$-halogenierten Ketonen mit Metallsulfiden des Typs $\mathrm{L}_{\mathrm{n}}-\mathrm{M}(\mathrm{SH})_{2}$. Bedingt durch die hohe Nukleophilie der Thiol-Gruppen können diese leicht durch die positivierten Kohlenstoff-Atome der Halogenketone alkyliert werden [90, 91, 92]. Die unter milden Bedingungen ablaufende Synthese (Abbildung 1.12) verspricht eine hohe Toleranz gegenüber $\mathrm{pH}$-empfindlichen funktionellen Gruppen so dass vor allem pyridinsubstituierte Dithiolenkomplexe erhalten werden konnten [93].

Ein wesentlicher Beitrag zu neuen Syntheserouten stammt von der Gruppe um Holm. Hierbei ist von Bedeutung, dass die Bildung inerter Trisdithiolenkomplexe verhindert wird. Dabei konnte zahlreiche neue Mo- und W-Dithiolenkomplexe charakterisiert und hinsichtlich ihrer Reaktivität untersucht werden [94, 95, 96, 97, 98, 99. 100, 101, 102, 103, 104, 105, 106].

So öffnet die Verwendung von $\left[\mathrm{Mo}^{\mathrm{IV}} \mathrm{OCl}(\mathrm{MeCN})_{4}\right]^{+}$und $\left[\mathrm{Mo}^{\mathrm{IV}} \mathrm{Cl}_{4}(\mathrm{MeCN})_{2}\right]$ als 
Abbildung 1.12: Umsetzung von Metallsulfiden
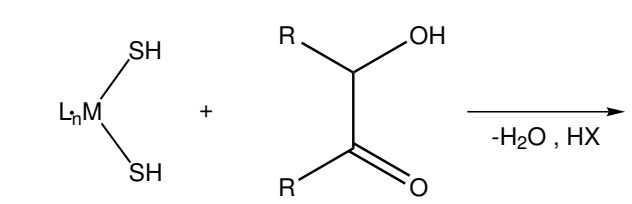<smiles>[R]C1=C(Br)SN=[As]1</smiles>

$\operatorname{LnM}=$

$\mathrm{M}\left(\mathrm{Ph}_{2} \mathrm{PC}_{2} \mathrm{H}_{4} \mathrm{PPh}_{2}\right)$

$\mathrm{X}=\mathrm{Br}$

$\mathrm{Cp}_{2} \mathrm{Mo}$

tosyl, OPO(OEt)

$\mathrm{R}_{1}=\mathrm{H}, \mathrm{Me}, \mathrm{Ph}$<smiles>[R]=[Fe]</smiles><smiles>Cc1cccnc1</smiles><smiles>Cc1ccccn1</smiles><smiles>Cc1cnc2ccccc2n1</smiles>

Metall-Vorstufen den Zugang zu quadratisch-pyramidalen $\left[\mathrm{Mo}^{\mathrm{IV}} \mathrm{O}\left(\mathrm{S}_{2} \mathrm{C}_{2} \mathrm{R}_{2}\right)_{2}\right]^{2-}$-Komplexen [96].

Eine vielversprechende Route zu Mo- und W-(bis-1,2)-Dithiolenkomplexen ist auch der Ligandenaustausch von $\mathrm{Mo}(\mathrm{MeCN})_{3}(\mathrm{CO})_{3}$ bzw. $\mathrm{W}(\mathrm{MeCN})_{3}(\mathrm{CO})_{3}$ mit $\mathrm{Ni}\left(\mathrm{S}_{2} \mathrm{C}_{2} \mathrm{R}_{2}\right)_{2}$ [99, 100, 102, 104, 105] welche zu den Bis-1,2-endithiolendicarbonyl-Komplexen $\mathrm{Mo}(\mathrm{CO})_{2}$ $\left(\mathrm{S}_{2} \mathrm{C}_{2} \mathrm{R}_{2}\right)_{2}$ bzw. W(CO) $)_{2}\left(\mathrm{~S}_{2} \mathrm{C}_{2} \mathrm{R}_{2}\right)_{2}$ führen. Diese Verbindungen stellen wertvolle Vorstufen für eine große Anzahl an Modellen für Vertreter der DMSOR-, Aldehyd Oxidoreduktasesowie der Formiatdehydrogenase-Familie dar.

\subsubsection{Synthese-Ansätze für das Pyranopterin-1,2-En-Dithiolen}

Bisher gibt es keinen kompletten Modellkomplex welcher über alle drei redoxaktiven Zentren - das Metall-Ion, die Dithioleneinheit und das hydrogenierte Pyranopterin verfügt.

Pionierarbeit auf dem Gebiet der Pyranopteridine, ihrer Synthese und Charakterisierung leisteten Pfleiderer et al. in den 90ern [107, 108].

Aufbauend auf diese Vorarbeiten entwickelten Burgmayer et al. [109] einen Ansatz, 
der von der in Abbildung 1.13 gezeigten Syntheseroute ausgeht. So gelang es, durch Addition einer an das Pteridin geknüpften Dreifachbindung an einen Persulfidokomplex einen Komplex, der ein geöffnetes Pterin enthält, zu erhalten.

Abbildung 1.13: Addition eines Pteridins an eine Dreifachbindung

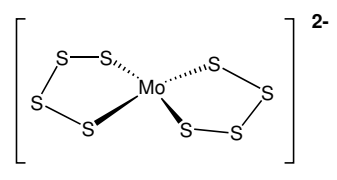<smiles>CC(=O)C#Cc1cnc2nc(NC(=O)C(C)(C)C)[nH]c(=O)c2n1</smiles>

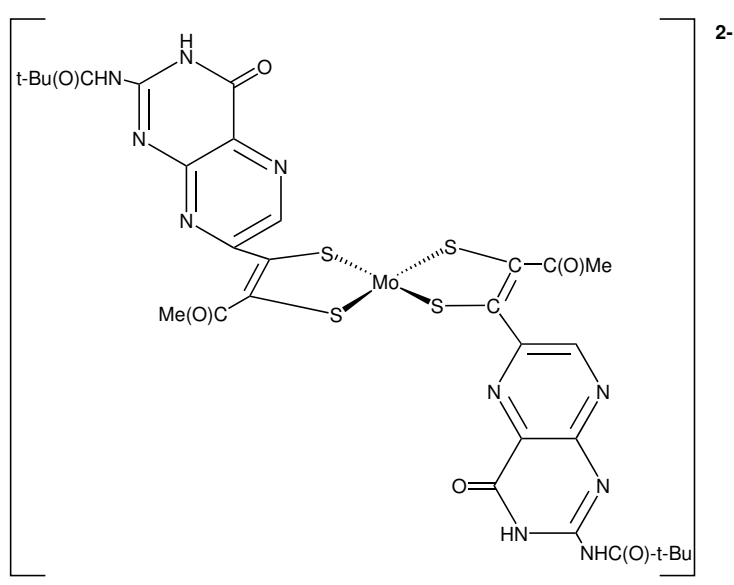

Abbildung 1.14: Offener Pterindithiolenkomplex von Garner et al.<smiles></smiles>

Mit dem Einsatz eines dithiocarbonatgeschützten Pterins entwickelten Garner und Joule einen anderen Weg und konnten so einen Pterindithiolenkomplex [51] erhalten. Die Schwierigkeiten, dieses anspruchsvolle Ligandensystem aufzubauen, zeigen sich deutlich daran, dass es bisher nur in der offenen Form synthetisiert werden konnte.

Später gelang es den Arbeitsgruppen von Garner und Joule mit der Synthese eines Pyrano-2,3-quinoxalindithiolens, welches als Cobalt-Komplex erhalten werden 
Abbildung 1.15: Cobalt-pyrano-2,3-quinoxalindithiolenkomplex von Garner und Joule

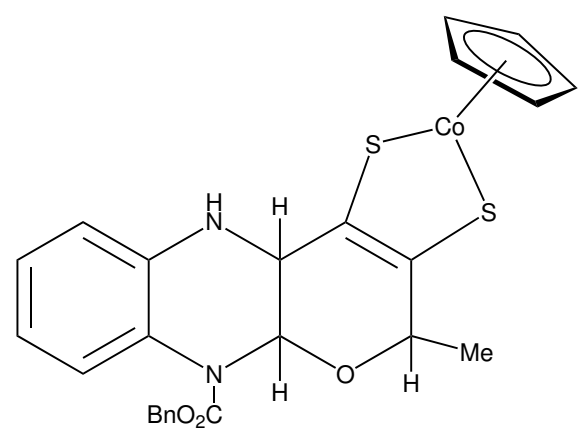

konnte, einen weiteren wesentlichen Beitrag für den Aufbau von Modellkomplexen zu liefern [110]. Dennoch gibt es bisher keinen Mo- bzw. Wolframkomplex, mit diesem anspruchsvollen Liganden.

\subsection{Aufgabenstellung}

In Anlehnung an den von Garner et al. veröffentlichten Weg [86] sollten Dithiolenkomplexe von Molybdän und Wolfram synthetisiert und isoliert werden, welche über einen sechsgliedrigen Pyranring neben der Dithioleneinheit verfügen. Weiterhin sollte das Metall dabei von zwei Liganden und einer Oxo-Gruppe umgeben sein.

Abbildung 1.16: Übersicht aller Ligandensysteme<smiles>SC1=C(S)CO[CH]1</smiles><smiles>[S-]C1=C(S)c2ccccc2OC1</smiles><smiles>[S-]C1=C([S-])C(c2ccccc2)Oc2ccccc21</smiles><smiles>[S-]C1=C([S-])C2NC=CNC2OC1</smiles><smiles>Nc1nc2c(c(=O)[nH]1)NC1C(S)=C(S)COC1N2</smiles>

Um einen tieferen Einblick in die beim Redoxvorgang ablaufenden Prozesse dieser Komplexe zu erhalten, sollten die Elektronentransferserien M(IV)-M(VI) ( $\mathrm{M}=$ 
Mo und W) mit verschiedenen Liganden mittels Dichtefunktionaltheorie untersucht sowie die elektronische Struktur aufgeklärt werden. Hierbei wurden Modellsysteme gewählt, die analog der DMSO Reduktase-Familie über eine quadratisch-pyramidale Koordination, ein axiales Sauerstoff-Atom sowie je zwei Dithiolen-Liganden verfügen.

Dabei reicht die Palette der Liganden vom kleinsten Vertreter, einem an die Dithioleneinheit folgenden Pyranring, über zwei mit aromatischem Rückgrat substituerte Dithiolenpyrane, einem Zweiringsystem, bei dem ein Pyrazinring an den Pyranring geknüpft ist, bis zu dem kompletten Kofaktor. 


\section{Kapitel 2}

\section{Ergebnisse und Diskussion}

Um ein tieferes Verständnis hinsichtlich des Redoxprozesses zu erlangen, wurden Komplexe in Anlehnung an die Struktur des aktiven Zentrums der DMSO ReduktaseFamilie mit theoretischen Methoden (Dichtefunktionaltheorie) untersucht. Dabei wurden jeweils Verbindungen von Molybdän und Wolfram mit den entsprechenden Liganden, in den auch im biologischen Prozess vorkommenden Oxidationsstufen IV, V und VI behandelt. Die jeweiligen Elektronentransferserien werden in den folgenden Kapiteln vorgestellt.

Die Behandlung des ersten Systems erfolgt hierbei besonders ausführlich, da es auch der Entwicklung einer gemeinsamen Behandlung dient. Die allen Systemen gemeinsamen Begrifflichkeiten und Charakteristika werden dann im folgenden nur noch durch Verweise gekennzeichnet.

Bei der Synthese der Modellkomplexe lag das Hauptaugenmerk auf der Darstellung von Liganden, die sowohl die Dithioleneinheit als auch den Pyranring beinhalten. Die Synthese der Liganden selbst sowie der Modellkomplexe ist der Diskussion der entsprechenden Systeme vorangestellt. 


\subsection{Das System $\left[\mathrm{MO}(\mathrm{pdt})_{2}\right]^{z-}$}

Bis auf wenige Ausnahmen, so dass von Sugimoto et al. [111] synthetisierte $\left[\mathrm{MoO}(\mathrm{pdt})_{2}\right]^{2-}$ sowie das von unserem Arbeitskreis publizierte Paar $\left[\mathrm{MO}(\mathrm{fdt})_{2}\right]^{2-}(\mathrm{M}=\mathrm{Mo}, \mathrm{W})[112]$ gibt es bisher keine Modellkomplexe, die eine an einen Pyranring verknüpfte Dithioleneinheit besitzen.

Die synthetische Herausforderung dieser Spezies besteht zum einen an der generell nicht leicht zugänglichen Dithiolen-Einheit, zum anderen kommt mit dem angeknüpften Pyran noch ein recht empfindliches aliphatisches Gerüst hinzu. Es erweist sich als angebracht, bereits einen geschlossenen Pyranring enthaltende Systeme als Vorstufen zu verwenden, da ein nachträglicher Ringschluss an einem derartigen System problematisch ist.

Die Auswahl an Vorstufen beschränkt sich somit auf Pyranoketone, welche aufgrund ihrer Ketofunktionalität als Edukte für die von Garner und Joule entwickelte Syntheseroute geeignet sind.

Eine Schwierigkeit dabei stellt die Umlagerung vom Xanthogenat bzw. Carbamat mittels einer starken Säure dar, die auch Auswirkungen auf die empfindliche Etherfunktionalität hat. Wegen dieser Problematik wurde eine neue Syntheseroute angewendet, bei der die Dithioleneinheit unter milden Bedingungen aufgebaut wird. Jedoch sind auch in dieser Route bei der Freisetzung des Dithiolens aus dem Dithiin drastischere Bedingungen erforderlich, die wiederum Auswirkungen auf das empfindliche Tetrahydropyran haben.

\subsubsection{Synthese von $\left[\mathrm{PPh}_{4}\right]_{2}\left[\mathrm{MoO}(\mathrm{pdt})_{2}\right]$}

Der einfachste Vertreter der Pyrano-2,3-Dithiolene, das 5,6-Dihydro-2H-pyran-3,4dithiolen, ist als kleinstes aliphatisches Modell bereits 2005 von Sugimoto et al. [111] 
synthetisiert und charakterisiert worden. Es gelang, der Syntheseroute von Holm [113] folgend, über eine Metallübertragung ausgehend von Nickeldithiolenkomplexen die entsprechenden Molybdänverbindungen mit verschiedenen aliphatischen Liganden zu synthetisieren.

Die in dieser Arbeit angewandte Synthesestrategie nach Garner et al. [86] führte durch die direkte Umsetzung des Dithiocarbonats mit den entsprechenden Metallvorstufen $\mathrm{K}_{3} \mathrm{Na}\left[\mathrm{MO}_{2}(\mathrm{CN})_{4}\right](\mathrm{M}=\mathrm{Mo}, \mathrm{W})$ nicht zum gewünschten Produkt, wenngleich es möglich war, das Dithiocarbonat auf diesem Weg zu erhalten.

Um der sehr empfindlichen Natur dieses Liganden gerecht zu werden, wurde eine andere Synthesestrategie entwickelt. Hierbei wurde, wie in Abbildung 2.1 gezeigt, in Anlehnung an eine Syntheseroute von Jekö et al. [114] das Pyranon mit 1,2Ethandithiol und katalytisch eingesetztem $\mathrm{BF}_{3}$-Etherat in das recht stabile Pyranthioketal 12 überführt. Das erhaltene NMR-Spektrum zeigt die Signale der vier Protonen der Thioketalbrücke als Singulett bei 3.23 ppm. Die Signale der sich am Pyranring befindenden Protonen sind alle zu einem Triplett aufgespalten und liegen bei den dem Sauerstoff benachbarten Protonen etwas tieffeldverschoben bei 3.68 ppm während die restlichen bei 2.03 ppm liegen. Das Ergebnis der Massenspektrometrie bestätigt das Ergebnis.

Das Thioketal 12 lässt sich durch Einsetzen einer Brom-Quelle und die dadurch ausgelöste Umlagerung in das Pyranodithiin 13 überführen. Hierbei wurde das Bromierungsmittel NBS verwendet.

Die von Jekö et al. synthetisierten Dithiine zeigten keine große Stabilität, auch das Pyranodithiin 13 zersetzte sich nach wenigen Minuten und wurde daher sofort weiterverarbeit. Daher konnte bis auf ein NMR-Spektrum keine weitere Analytik durchgeführt werden. Das NMR-Spektrum zeigt eine leichte Tieffeldverschiebung aller Protonen im Vergleich zum Thioketal. Die Signale der sich am Dithiin-Ring befindenden 
Abbildung 2.1: Syntheseroute über das Dithiin<smiles>O=C1CCOCC1</smiles>

11

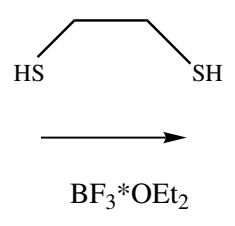

$\mathrm{BF}_{3} * \mathrm{OEt}_{2}$

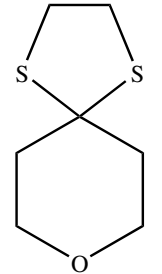

12

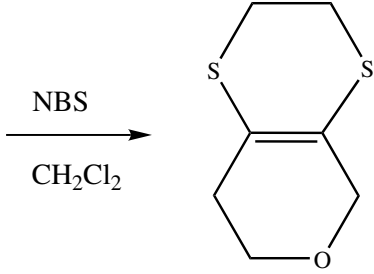

13
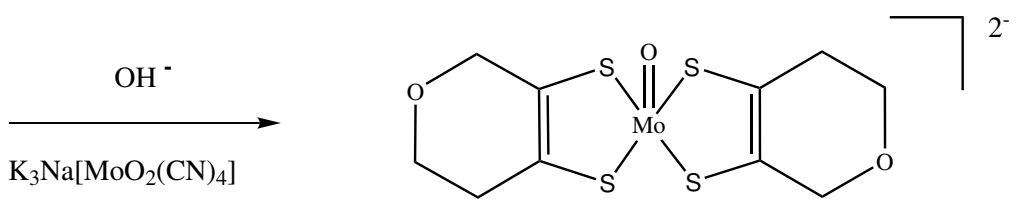

$1 \mathbf{a}$

Protonen liegen bei 3.29 ppm, die beiden neben dem Dithiinring liegenden Protonen erscheinen nun als Singulett bei 3.95 ppm während die auf der anderen Seite des Pyranrings liegenden Protonen wiederum zu Tripletts aufspalten und bei 2.08 und 3.73 ppm erscheinen.

Bei der basischen Spaltung des Dithiins zum Dithiolen ergibt sich auch hier das Problem, die Bedingungen für die Thioetherspaltung neben der Etherfunktionalität des Pyranrings zu optimieren. Thioether werden leichter gespalten als Oxoether, jedoch können die Bedingungen für die Etherspaltung im allgemeinen nicht so fein abgestimmt werden um beiden gerecht zu werden. Die basischen Bedingungen sind bei der Metallumsetzung im Fall des Pyrans gerade noch ausreichend um die EthanEinheit des Dithiins abzuspalten während die aromatischen Analoga, Chromanyl- und Flavanyldithiin, nicht gespalten werden können. Der Einsatz von Na in HMPA greift jedoch auch die Etherfunktion an und führt so zur teilweisen Zersetzung des gesamten Rings, was die Ausbeute negativ beeinflusst.

Aufgrund der leicht öligen Konsistenz des erhaltenen Metallkomplexes 1a konnten keine für die Röntenstrukturanalyse geeigneten Kristalle gewonnen werden. Die 
Abbildung 2.2: Struktur von 1a

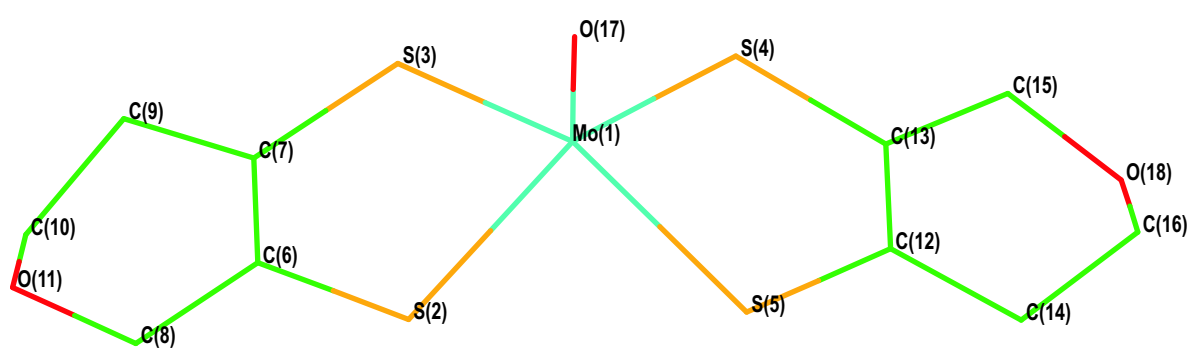

erhaltene Elementaranalyse bestätigt jedoch seine Zusammensetzung.

Das IR-Spektrum zeigt die Mo-O-Streckschwingung mit hoher Intensität bei 1071 $\mathrm{cm}^{-1}$. Im Vergleich mit der für diesen Komplex berechneten Schwingung von $947 \mathrm{~cm}^{-1}$ ergibt sich recht großer Unterschied, der darauf zurückzuführen ist, dass hierbei nur die harmonischen Schwingungsfrequenzen berechnet wurden. Die Berücksichtigung anharmonischer Effekte ist für Systeme dieser Größenordnung nicht möglich. Der aus diesem Grund eingeführte Skalierungsfaktor $f=\frac{\nu_{\text {theo }}}{\nu_{\text {exp }}}$ ergibt sich hier zu 0.88, was in guter Übereinstimmung mit den Ergebnissen der Chromanyl- und Flavanylkomplexe des folgenden Kapitels ist. Auch folgen die übrigen theoretischen und berechneten Banden diesem Verhältnis.

\subsubsection{Geometrische Strukturen}

Die Struktur der untersuchten Komplexe $\left[\mathrm{MO}(\mathrm{pdt})_{2}\right]^{\mathrm{z}-}(\mathrm{M}=\mathrm{Mo}, \mathrm{W}$; pdt $=5,6$ Dithydro-2 $H$-pyran-3,4-dithiolen, $\mathrm{z}=-2,-1,0)$ ist exemplarisch anhand der Verbindung 1a in Abbildung 2.2 dargestellt. Hierbei steht 1a-c für die Verbindungen $\left[\mathrm{MoO}(\mathrm{pdt})_{2}\right]^{z}$ mit $\mathrm{z}=-2,-1,0$ sowie $2 \mathrm{a}-\mathrm{c}$ für die Verbindungen $\left[\mathrm{WO}(\mathrm{pdt})_{2}\right]^{z}$ mit $\mathrm{z}=$ $-2,-1,0$.

Im Gegensatz zu den quadratisch-pyramidalen Bis-Dithiolenkomplexen [99] ändert sich die Geometrie bei den Oxo-Komplexen in Bezug auf das Metallzentrum drastisch (Abbildung(2.3). Das Sauerstoff-Atom steht an der Spitze des Metallzentrums während 
Abbildung 2.3: Strukturen von $\left[\mathrm{MoO}(\mathrm{pdt})_{2}\right]^{\mathrm{z}-}(\mathrm{z}=-2,-1,0)$
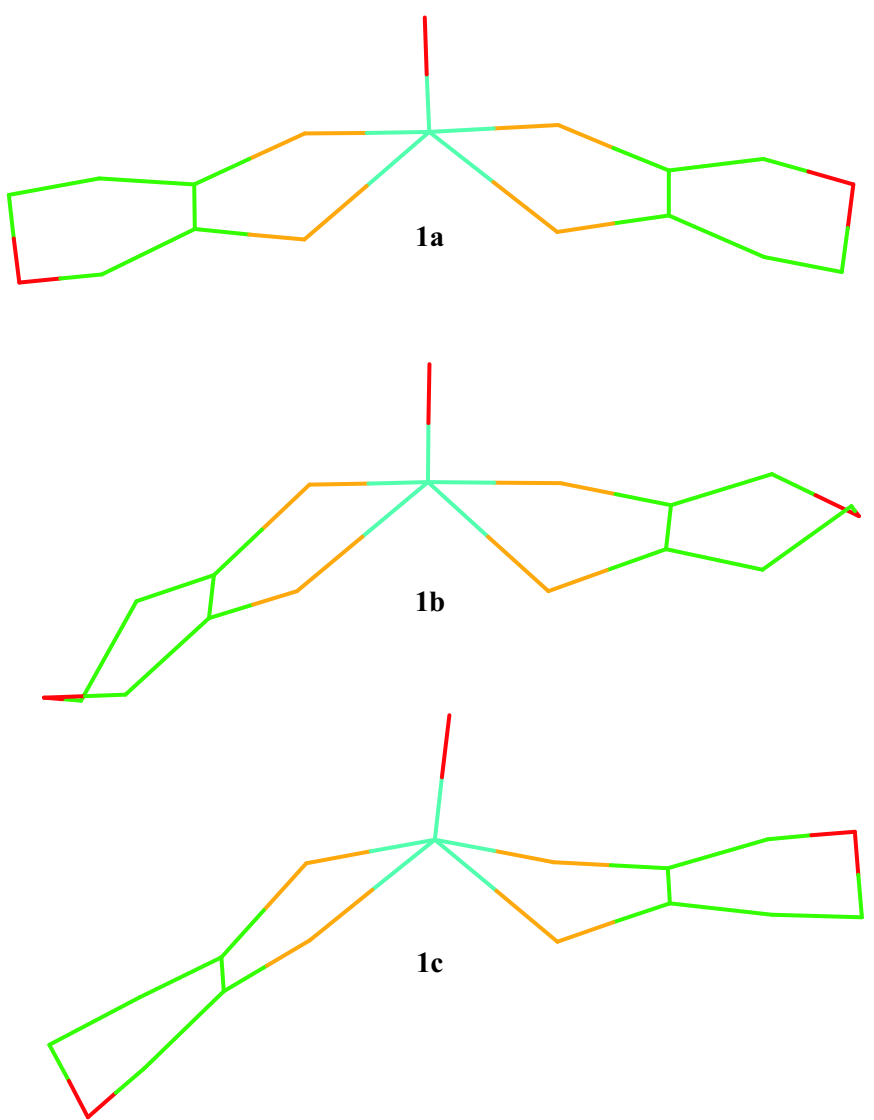

das Metall über die von den vier Schwefelatomen gebildete Ebene angehoben wird.

Der berechnete Wert für den Abstand des Molybdäns von der Ebene für 1a ist mit $0.756 \AA$ in sehr guter Übereinstimmung mit dem experimentell gefundenen Wert von $0.76 \AA$ [11]. Selbst der Vergleich mit dem bei der Struktur der Arsenitoxidase bestimmten Wert von $0.8 \AA$ zeigt, dass diese Modellkomplexe die Situation am Metallzentrum gut widerspiegeln.

In Tabelle 2.1] sind die wesentlichen das Metallzentrum betreffenden Geometrieparameter aufgeführt, Abbildung 2.2 zeigt die dazu gehörige nummerierte Struktur.

Vergleicht man beide Metalle miteinander, zeigt sich bei Wolfram eine weniger große Entfernung des Metalls von der Ebene. Dies ist auch beim Durchlaufen der Oxidation, bei der dieser Wert beim Molybdän stärker zunimmt, zu sehen. So vergrö- 
Tabelle 2.1: Ausgewählte Geometrieparameter der Verbindungen $\left[\mathrm{MO}(\mathrm{pdt})_{2}\right]^{z-}, \mathrm{M}=$ Mo, W; $\mathrm{z}=-2,-1,0$

\begin{tabular}{lcccccc}
\hline & $\mathbf{1 a}$ & $\mathbf{1 b}$ & $\mathbf{1 c}$ & $\mathbf{2 a}$ & $\mathbf{2 b}$ & $\mathbf{2 c}$ \\
\hline $\mathrm{M}(1)-\mathrm{S}_{4}{ }^{a}$ & $0.756(4)$ & $0.808(4)$ & $0.847(8)$ & $0.727(8)$ & $0.766(5)$ & $0.787(3)$ \\
$\mathrm{M}(1)-\mathrm{O}(17)$ & 1.7127 & 1.7075 & 1.6981 & 1.7237 & 1.7153 & 1.7057 \\
$\mathrm{M}(1)-\mathrm{S}(2)$ & 2.4735 & 2.4489 & 2.4025 & 2.4528 & 2.4398 & 2.3918 \\
$\mathrm{M}(1)-\mathrm{S}(3)$ & 2.4687 & 2.4466 & 2.4023 & 2.4483 & 2.4375 & 2.3926 \\
$\mathrm{M}(1)-\mathrm{S}(4)$ & 2.4727 & 2.4351 & 2.4297 & 2.4516 & 2.4320 & 2.4179 \\
$\mathrm{M}(1)-\mathrm{S}(5)$ & 2.4710 & 2.4320 & 2.4266 & 2.4506 & 2.4298 & 2.4161 \\
$\mathrm{~S}(2)-\mathrm{C}(6)$ & 1.7829 & 1.7601 & 1.7448 & 1.7904 & 1.7695 & 1.7570 \\
$\mathrm{~S}(3)-\mathrm{C}(7)$ & 1.7831 & 1.7627 & 1.7451 & 1.7904 & 1.7717 & 1.7566 \\
$\mathrm{~S}(4)-\mathrm{C}(13)$ & 1.7825 & 1.7707 & 1.7257 & 1.7900 & 1.7754 & 1.7397 \\
$\mathrm{~S}(5)-\mathrm{C}(12)$ & 1.7834 & 1.7735 & 1.7293 & 1.7906 & 1.7785 & 1.7433 \\
$\mathrm{C}(6)-\mathrm{C}(7)$ & 1.3426 & 1.3481 & 1.3754 & 1.3401 & 1.3425 & 1.3684 \\
$\mathrm{C}(12)-\mathrm{C}(13)$ & 1.3426 & 1.3489 & 1.3716 & 1.3401 & 1.3467 & 1.3614 \\
$\mathrm{O}(17)-\mathrm{M}(1)-[\mathrm{S}(2)-\mathrm{S}(3)]$ & 113.92 & 117.88 & 112.55 & 113.21 & 116.46 & 111.13 \\
$\mathrm{O}(17)-\mathrm{M}(1)-[\mathrm{S}(4)-\mathrm{S}(5)]$ & 113.94 & 114.13 & 123.19 & 113.19 & 113.11 & 121.24 \\
$\mathrm{FW}{ }^{b} \mathrm{M}(1)-\mathrm{S}(2)-\mathrm{S}(3)$ & 13.81 & 8.96 & 14.80 & 12.64 & 2.88 & 18.82 \\
$\mathrm{FW}{ }^{b} \mathrm{M}(1)-\mathrm{S}(4)-\mathrm{S}(5)$ & 14.38 & 31.72 & 37.17 & 13.12 & 32.21 & 41.14 \\
$\mathrm{C}(8)-\mathrm{O}(11)$ & 1.4199 & 1.4205 & 1.4208 & 1.4202 & 1.4208 & 1.4210 \\
$\mathrm{C}(10)-\mathrm{O}(8)$ & 1.4373 & 1.4246 & 1.4138 & 1.4381 & 1.4249 & 1.4140 \\
$\mathrm{C}(15)-\mathrm{O}(18)$ & 1.4372 & 1.4251 & 1.4134 & 1.4379 & 1.4248 & 1.4137 \\
$\mathrm{C}(16)-\mathrm{O}(18)$ & 1.4197 & 1.4207 & 1.4212 & 1.4201 & 1.4210 & 1.4216 \\
$\mathrm{C}(7)-\mathrm{C}(6)-\mathrm{C}(8)-\mathrm{O}(11)$ & -9.64 & -13.08 & -12.00 & -9.35 & -12.71 & -10.97 \\
$\mathrm{C}(12)-\mathrm{C}(13)-\mathrm{C}(15)-\mathrm{O}(18)$ & 10.91 & 13.59 & 14.15 & 10.74 & 13.43 & 13.22 \\
& & & & & & \\
${ }^{a} \mathrm{~S}_{4}$ beschreibt die von den vier S-Atomen aufgespannte Ebene & & \\
${ }^{b} \mathrm{FW}=\mathrm{Faltungswinkel}$ zwischen dem Metall & und dem & Vektor beider Schwefel-Atome
\end{tabular}

ßert sich der Abstand bei den Molybdänkomplexen auf $0.847 \AA$ während sich bei den Wolframkomplexen eine Zunahme von $0.727 \AA$ auf $0.787 \AA$ ergibt.

Ein ganz wesentlicher Trend beim Durchlaufen der Einelektronenschritte zeigt sich beim Verhalten der Liganden. Das nahezu symmetrische Erscheinungsbild bei den Komplexen (1a und 2a, M = Mo und W) ändert sich drastisch beim Wechsel der Oxidationsstufe zu M(V) und $\mathrm{M}(\mathrm{VI})$.

Während ein Ligand bezogen auf den S-S-Vektor in seiner Ursprungslage nur ge- 
Abbildung 2.4: Definition des Faltungswinkels

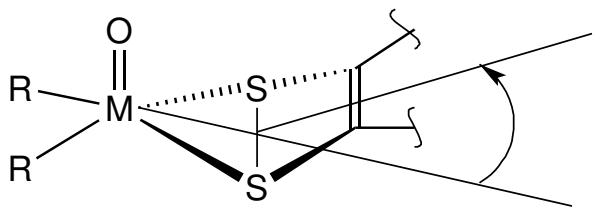

ringfügig schwankt 1 , wird der andere beim Wechsel in OS V stark (Mo: $14.38^{\circ}$ auf $31.72^{\circ}, \mathrm{W}: 13.12^{\circ}$ auf $32.21^{\circ}$ ) und in OS VI nochmals um einen geringeren Betrag (Mo: $31.72^{\circ}$ auf $37.17^{\circ}$, W: $32.21^{\circ}$ auf $41.14^{\circ}$ ) angehoben. Das Auftreten dieses sogenannten Faltungswinkels des fünfgliedrigen Dithiolen-Chelats wurde bei verschiedenen Komplexen [115] beobachtet und wird durch die unterschiedliche Elektronenkonfiguration am Metall verursacht.

Betrachtet man dabei den apikalen Sauerstoff O(17) im Verhältnis zur S(4)-S(5)Linie, zeigt sich, dass der eingeschlossene Winkel von ursprünglich $113.92^{\circ}$ (Mo, W $113.94^{\circ}$ ) auf $117.88^{\circ}\left(\mathrm{Mo}, \mathrm{W} 116.46^{\circ}\right)$ und schließlich auf $123.19^{\circ}$ (Mo, W $121.24^{\circ}$ ) zunimmt. Der Winkel bezüglich des anderen Liganden bleibt jedoch weitgehend konstant.

Der Metall-Sauerstoff-Bindungsabstand verkürzt sich dabei bei beiden Metallen um $0.015 \AA$ (Mo) bzw. $0.018 \AA(\mathrm{W})$ von ursprünglichen $1.7127 \AA$ (Mo) bzw. 1.7237 $\AA$ (W). Der Wert für die Mo(IV)-Verbindung 1a liegt dabei etwas unter dem experimentellen Vergleichswert von 1.745(6) $\AA$.

Die durchschnittlichen Abstände M-S liegen mit Werten zwischen $2.39 \AA$ (2c) bis $2.47 \AA$ (1a) über den experimentell bestimmten Bindungslängen von durchschnittlich $2.37 \AA$ im von Sugimoto et al. 111 kristallographisch untersuchten analogen Molybdänkomplex sowie auch über dem zu $2.37 \AA$ bestimmten Wert in der reduzierten Form der Arsenitoxidase [116].

\footnotetext{
${ }^{1}$ Absinken in OS V und erneutes Ansteigen auf den Ursprungswert in OS VI
} 
Generell werden Metall-Schwefel-Bindungen von der Dichtefunktionalmethode überschätzt und sind aufgrund systematischer Natur zu lang [117]. Der dennoch erkennbare Trend zeigt, dass sich die vier Metall-Schwefel-Bindungen bei Erreichen der OS (V) und OS(VI) unterschiedlich verhalten. Bei den Komplexen 1a und 2a sind die vier Bindungen fast gleich lang und es gibt nur unmerkliche Unterschiede die zwischen 0.001 bis $0.005 \AA$ liegen. In den OS V und VI sind jeweils die beiden Metall-SchwefelBindungen die zu einem Liganden gehören nahezu gleich lang. In der OS V unterscheiden sie sich um etwa $0.1 \AA$ und in der OS VI um etwa $0.2 \AA$. Dabei sind die Bindungen auf der Seite des gefalteten Liganden kürzer (M(1)-S(4) und M(1)-S(5)). Der Vergleich beider Metalle zeigt, dass die Wolfram-Schwefel-Bindungen generell kürzer (in der Größenordung von 0.1 bis $0.2 \AA$ ) als die Molybdän-Schwefel-Bindungen ausfallen. Die Erhöhung der OS führt zu einer gleichmäßigen Verkürzung um jeweils $0.2 \AA$ bei beiden Oxidationsschritten.

Der Abstand der Schwefel-Atome zu den benachbarten Doppelbindungen nimmt beim Oxidationsprozess stark ab; in OS IV beträgt er durchschnittlich $1.783 \AA$ beim Mo (1.790 $\AA$ bei W), in OS VI dagegen nur noch $1.73 \AA$ (W $1.74 \AA)$. Dagegen beobachtet man bei der C-C-Doppelbindung eine Zunahme der Bindungslänge von 1.343 A auf $1.374 \AA$ beim Mo (W 1.340 auf $1.365 \AA$ ). Diese Werte liegen oberhalb des Bereiches für die C-C-Doppelbindung im Ethen, die $1.33 \AA$ beträgt [118]. In Hinblick auf die Elektronendichte an der C-C-Doppelbindung entspricht die Zunahme der Bindungslänge auch einer Zunahme an Delokalisierung. Im Vergleich zu den Bindungslängen im unsubstituierten Dithiolenliganden edt $^{z-}$ (edt $=1,2$-Ethylendithiolen $)$ zeigen sich ähnliche Trends.

Die Schwefel-Kohlenstoff-Bindung nimmt hier allerdings von $1.789 \AA$ im DithiolenDianion über 1.770 $\AA$ im Monoanion bis auf $1.629 \AA$ im ungeladenen Dithiolen ab. Die Schwefel-Kohlenstoff-Bindungen in den Komplexen der OS IV und V stimmen sehr gut 
Tabelle 2.2: Bindungslängen im unsubstituierten Dithiolenliganden (edt) ${ }^{z-}$

\begin{tabular}{cccc}
\hline & $\mathrm{C}_{2} \mathrm{~S}_{2}{ }^{2-}$ & $\mathrm{C}_{2} \mathrm{~S}_{2}{ }^{1-}$ & $\mathrm{C}_{2} \mathrm{~S}_{2}$ \\
\hline $\mathrm{C}-\mathrm{S}$ & 1.7888 & 1.7690 & 1.6292 \\
$\mathrm{C}=\mathrm{C}$ & 1.3591 & 1.3380 & 1.4704
\end{tabular}

mit den Bindungslängen im freien Dithiolen überein. Der non-innocent Charakter der Dithioleneinheit kommt also auch in den Komplexen zum Tragen. Dies gilt auch für die C-C-Doppelbindung, die sowohl im Ethylendithiolen als auch im Komplex nahezu gleiche Werte für den Fall der niedrigsten und mittleren OS aufweist. Lediglich bei der höchsten OS ergeben sich recht hohe Abweichungen von etwa $0.1 \AA$, wobei die SC-Bindung im Komplex um diesen Betrag länger und die C-C-Doppelbindung kürzer ist. Das deutet darauf hin, dass der Ligand in dieser Oxidationsstufe nicht als DithietForm vorliegt, sondern eher der Monoanion-Form entspricht und die Elektronenabgabe nicht ausschließlich vom Dithiolen getragen wird.

Der Einfluss des Redoxprozesses reicht jedoch bis in den Pyranring, wie an der Änderung der C-O-Bindungslänge zu sehen ist. Die Änderungen sind zwar sehr gering, aber zeigen, dass das gesamte Molekül, wenn auch mit abnehmender Wirkung je weiter die betreffenden Atome vom Metall entfernt sind, in den Redoxprozess involviert ist.

Die näher an der Dithioleneinheit liegende Bindung C(12)-O(17)2 2 verlängert sich marginal von $1.4199 \AA$ auf $1.4212 \AA$ (Mo ) bzw. von $1.4202 \AA$ auf $1.4216 \AA(\mathrm{W})$. Die zur anderen Seite liegende Bindung C(8)-O(17) dagegen wird mit zunehmender Oxidation verküzt und zwar in stärkerem Maße. So nimmt die Bindung von $1.4373 \AA$ auf $1.4134 \AA$ (Mo) bzw. von $1.4381 \AA$ auf $1.4137 \AA(\mathrm{W})$ ab.

Weitere Veränderungen sind auch an der Stellung des Pyran-Sauerstoffs zu erkennen. Die eingenommene Position (endo vs. exo) scheint keinem Trend zu unterliegen, da das System ohne weiteren angebundenen Ring keine Einschränkungen der Konfor-

\footnotetext{
${ }^{2}$ Änderung bei beiden Liganden identisch
} 
Tabelle 2.3: NPA Ladungen

\begin{tabular}{ccccccc}
\hline & $1 \mathrm{a}$ & $1 \mathrm{~b}$ & $1 \mathrm{c}$ & $2 \mathrm{a}$ & $2 \mathrm{~b}$ & $2 \mathrm{c}$ \\
\hline $\mathrm{M}(1)$ & 0.55 & 0.71 & 0.60 & 0.68 & 0.96 & 0.88 \\
$\mathrm{O}(17)$ & -0.58 & -0.54 & -0.47 & -0.68 & -0.64 & -0.57 \\
$\mathrm{~S}(2)$ & -0.23 & -0.09 & 0.11 & -0.24 & -0.13 & 0.06 \\
$\mathrm{~S}(3)$ & -0.22 & -0.08 & 0.11 & -0.23 & -0.13 & 0.06 \\
$\mathrm{~S}(4)$ & -0.23 & -0.10 & 0.08 & -0.24 & -0.13 & 0.04 \\
$\mathrm{~S}(5)$ & -0.22 & -0.09 & 0.08 & -0.23 & -0.13 & 0.05 \\
$\mathrm{C}(6)$ & -0.24 & -0.23 & -0.20 & -0.24 & -0.22 & -0.20 \\
$\mathrm{C}(7)$ & -0.21 & -0.20 & -0.17 & -0.20 & -0.19 & -0.17 \\
$\mathrm{C}(12)$ & -0.21 & -0.19 & -0.16 & -0.20 & -0.19 & -0.16 \\
$\mathrm{C}(13)$ & -0.24 & -0.22 & -0.20 & -0.24 & -0.22 & -0.19
\end{tabular}

mation bedingt. So ist die Position des Pyransauerstoffs bei beiden Metallen und bei den beiden Liganden gleichermaßen in den OS IV und V endo, in OS VI dagegen exo. Dargestellt durch den Diederwinkel C(7)-C(6)-C(8)-O(17), welcher die Anhebung des Sauerstoffs gegenüber der von der C-C-Doppelbindung gebildeten Ebene beschreibt, ist ein deutlicher Anstieg des Sauerstoffs aus der Ringebene mit zunehmender Oxidation zu beobachten.

So steigt der Diederwinke $3^{3}$ von $9.64^{\circ}$ in OS IV über $13.08^{\circ}$ in OS V auf $14.15^{\circ}$ in OS VI. Die analogen Werte für die Wolframverbindungen liegen etwas darunter, folgen aber dem gleichen Trend.

\subsubsection{Elektronische Strukur und Bindung}

Die anhand einer NPA-Analyset bestimmten Werte für die Ladungen der einzelnen Atome sind in Tabelle 2.14 dargestellt. Die Änderung der Ladungen ist besonders am jeweiligen Metall sowie den direkt angrenzenden Atomen stark ausgeprägt während die an die C-C-Doppelbindung angrenzenden Atome weniger in den Ladungstransfer involviert sind.

\footnotetext{
${ }^{3}$ Betrachtet werden die Beträge der Winkel.

${ }^{4}$ NPA natural population analysis, "natürliche Populationsanalyse"
} 
Die Metall-Atome, welche als Elektronenakzeptoren fungieren, weisen positive Ladungen im Bereich von 0.5 bis 1 auf, d.h. dass Ladung von den Liganden auf die Metalle übertragen wird. Formal betrachtet werden von den Liganden etwa 3.5 Elektronen in den formalen OS des Metalls OS IV , 4.5 in der OS V und 5.5 Elektronen in der OS VI auf das Metall übertragen. Dieser Effekt ist beim Wolfram etwas stärker ausgeprägt als beim Molybdän. Beide Metalle unterscheiden sich in der Ladung um 0.2 Einheiten 5 zugunsten einer positiveren Ladung beim Wolfram. Beim Vergleich der verschiedenen OS ist festzustellen, dass sich die Ladung beim Wechsel von OS IV zu OS V erhöht und in OS VI wieder leicht absinkt. Bei der Betrachtung der Nachbaratome fällt auf, dass sowohl beim Oxo-Liganden als auch beim Schwefel der Dithiolenfunktion der negative Ladungsanteil abnimmt. Dieser Trend ist bei den Schwefel-Atomen deutlicher ausgeprägt als beim Sauerstoff-Atom. So steigt die Ladung am Schwefel mit Werten von -0.22 auf 0.11 bei den Mo-Komplexen (-0.23 auf 0.05 bei den WKomplexen) sogar bis in den positiven Bereich. Die Ladung am Sauerstoff verändert sich dagegen nur um Ladungseinheiten im Bereich von 0.1. Wird dabei berücksichtigt, dass pro Molekül vier Schwefel-Atome jedoch nur ein Sauerstoff-Atom beteiligt sind ergibt sich für die Dithiolen-Schwefel ein recht hoher Ladungstransfer, der wiederum dem non-innocent-Charakter geschuldet ist.

In Analogie zur Geometrie zeigt sich auch hier ein unsymmetrisches Verhalten obwohl die Unterschiede nur marginal ausfallen. In OS IV unterscheidet sich die Ladung unter den Schwefel-Atomen insofern, als dass die jeweils zueinander trans-ständigen Atome einen gleich hohen Wert haben. In den OS IV und VI sind jeweils die beiden zu einer Dithiolen-Einheit gehörenden Schwefel-Atome gleich stark geladen.

Um einen näheren Einblick in die Charakteristik der Bindungen am Metall zu gelangen, wurde eine Orbitalanalyse6 [119] der natürlich lokalisierten Molekülorbitale

\footnotetext{
${ }^{5}$ atomare Einheiten [a.u.]

${ }^{6}$ Analyse der natürlich lokalisierten Orbitale der Bindungen, NBO
} 
Abbildung 2.5: Anteile der M-O-Dreifachbindung
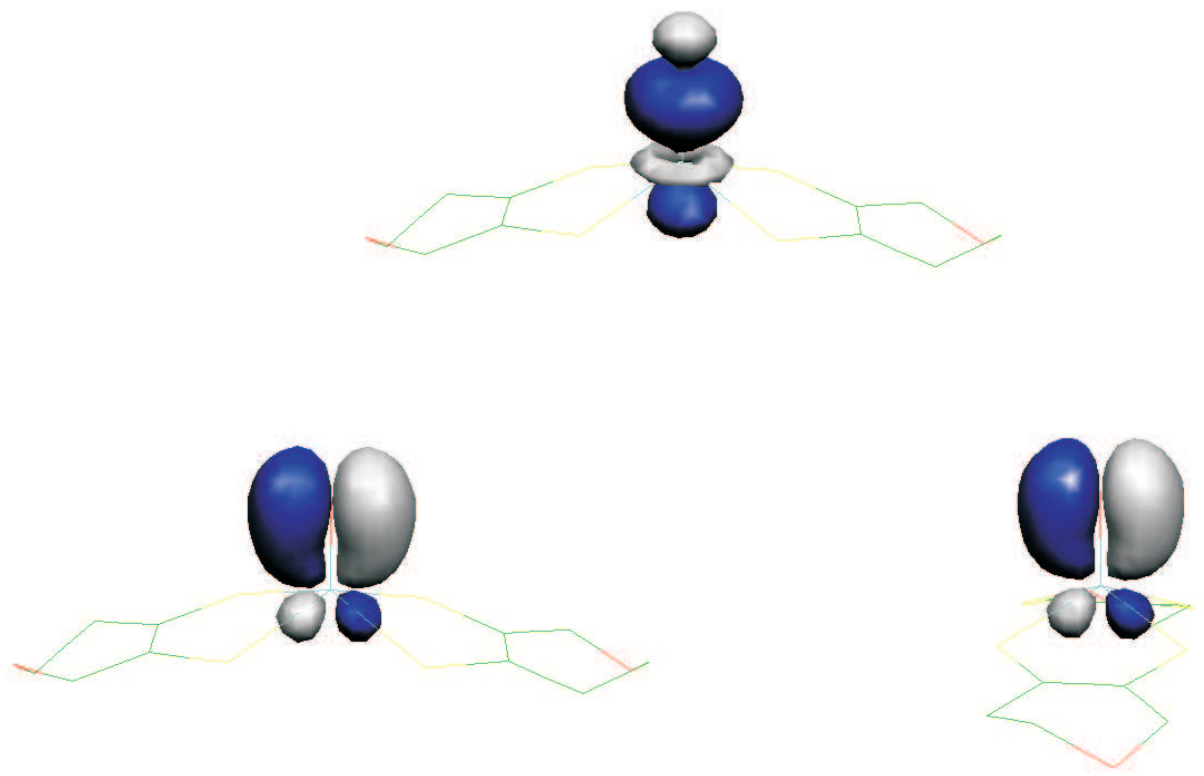

durchgeführt. Hierbei wurden die die Bindung bildenden Molekülorbitale in Hinsicht auf ihre Zusammensetzung analysiert. In den Tabellen 2.4 und 2.5] sind die das Metallzentrum betreffenden Bindungen der Komplexe in den OS IV, V und VI dargestellt. Tabelle 2.4 beinhaltet die Daten der Molybdänverbindungen, Tabelle 2.5 die der Wolframverbindungen. Dargestellt sind die an den Bindungen beteiligten Partner mit ihren Anteilen an der Bindung (Polarisation) sowie die am Bindungsatom lokalisierten Orbitale. Des weiteren wurde der Bindungsindex nach Wiberg (WBI) [120] bestimmt und ist bei der jeweiligen Bindung mit angegeben.

Die auf diese Weise erhaltene Analyse der Ladungsverteilung zeigt bei allen Verbindungen eine Metall-Sauerstoff-Dreifachbindung als optimale Lewis-Struktur. Dabei wird eines der beiden freien Elektronenpaare des Sauerstoffatoms in die Bindung zum Metall involviert. Die resultierende Dreifachbindung besteht jeweils aus einer $\sigma$-Bindung und zwei nahezu entarteten $\pi$-Bindungen, wie in Abbildung 2.5 gezeigt.

Die Metall-Sauerstoff- $\sigma$-Bindung resultiert aus einer Überlappung eines fast rei- 
Tabelle 2.4: Ausgewählte Bindungsparameter für $\left[\mathrm{MoO}(\mathrm{pdt})_{2}\right]^{z-}, \mathrm{z}=-2,-1,0$

\begin{tabular}{lllllllllll}
\hline A-B & Typ & $\% \mathrm{~A}^{a, b}$ & \multicolumn{3}{c}{ Hybridisierung } & $\%$ B & \multicolumn{3}{c}{ Hybridisierung } & $\mathrm{WBI}^{c}$ \\
& & & $\mathrm{~s}$ & $\mathrm{p}$ & $\mathrm{d}$ & & $\mathrm{s}$ & $\mathrm{p}$ & $\mathrm{d}$ & \\
\hline
\end{tabular}

$$
\left[\mathrm{Mo}^{I V} \mathrm{O}(\mathrm{pdt})_{2}\right]^{2-}
$$

$\begin{array}{lcccccccccc}\text { Mo-S(2) } & \sigma & 20.58 & 13.47 & 0.43 & 86.10 & 76.53 & 19.90 & 80.01 & 0.10 & 0.65 \\ \text { Mo-S(3) } & \sigma & 20.08 & 12.78 & 0.46 & 86.76 & 76.79 & 19.88 & 80.03 & 0.09 & 0.66 \\ \text { Mo-S(4) } & \sigma & 19.81 & 12.77 & 0.47 & 86.76 & 76.91 & 19.92 & 79.98 & 0.09 & 0.65 \\ \text { Mo-S(5) } & \sigma & 20.86 & 13.59 & 0.42 & 85.99 & 76.44 & 19.85 & 80.05 & 0.10 & 0.66 \\ \text { Mo-O(17) } & \sigma & 26.41 & 2.26 & 0.95 & 96.79 & 73.20 & 23.49 & 76.44 & 0.07 & \\ \text { Mo-O(17) } & \pi & 24.03 & 0.00 & 0.32 & 99.68 & 75.37 & 0.00 & 99.95 & 0.05 & 1.78 \\ \text { Mo-O(17) } & \pi & 24.66 & 0.00 & 0.31 & 99.69 & 74.71 & 0.00 & 99.95 & 0.05 & \end{array}$

$$
\left[\mathrm{Mo}^{V} \mathrm{O}(\mathrm{pdt})_{2}\right]^{1-}
$$

$\begin{array}{lcccccccccc}\text { Mo-S(2) } & \sigma & 22.661 & 11.18 & 0.76 & 88.06 & 73.51 & 16.17 & 83.71 & 0.12 & 0.70 \\ \text { Mo-S(3) } & \sigma & 23.53 & 10.78 & 0.83 & 88.39 & 72.94 & 16.13 & 83.74 & 0.13 & 0.71 \\ \text { Mo-S(4) } & \sigma & 25.61 & 12.96 & 0.58 & 86.45 & 71.48 & 16.15 & 83.70 & 0.15 & 0.73 \\ \text { Mo-S(5) } & \sigma & 26.28 & 12.78 & 0.66 & 86.56 & 71.06 & 16.21 & 83.63 & 0.16 & 0.74 \\ \text { Mo-O(17) } & \sigma & 28.21 & 2.01 & 0.79 & 97.21 & 71.43 & 21.51 & 78.41 & 0.07 & \\ \text { Mo-O(17) } & \pi & 23.74 & 0.02 & 0.21 & 99.77 & 74.82 & 0.19 & 99.76 & 0.05 & 1.75 \\ \text { Mo-O(17) } & \pi & 25.60 & 0.00 & 0.20 & 99.80 & 72.95 & 0.00 & 99.94 & 0.06 & \end{array}$

$$
\left[\mathrm{Mo}^{V I} \mathrm{O}(\mathrm{pdt})_{2}\right]
$$

$\begin{array}{llccccccccc}\text { Mo-S(2) } & \sigma & 24.39 & 12.06 & 0.66 & 87.28 & 72.54 & 16.04 & 83.82 & 0.14 & 0.79 \\ \text { Mo-S(2) } & \pi & 41.25 & 0.04 & 0.12 & 99.84 & 53.16 & 0.22 & 99.69 & 0.10 & \\ \text { Mo-C(6) } & \pi & & & & & <2 & & & & \\ \text { Mo-C(7) } & \pi & & & & & <2 & & & & \\ \text { Mo-S(3) } & \sigma & 24.54 & 11.78 & 0.79 & 87.43 & 72.35 & 16.16 & 83.69 & 0.15 & 0.80 \\ \text { Mo-S(4) } & \sigma & 25.25 & 11.71 & 0.83 & 87.46 & 71.57 & 15.19 & 84.64 & 0.17 & 0.81 \\ \text { Mo-S(5) } & \sigma & 26.06 & 12.00 & 0.91 & 87.09 & 70.99 & 15.20 & 84.61 & 0.19 & 0.82 \\ \text { Mo-O(17) } & \sigma & 24.86 & 0.01 & 0.11 & 99.88 & 73.33 & 0.00 & 99.94 & 0.06 & \\ \text { Mo-O(17) } & \pi & 25.18 & 0.01 & 0.13 & 99.86 & 73.08 & 0.01 & 99.93 & 0.06 & 1.80 \\ \text { Mo-O(17) } & \pi & 29.16 & 1.91 & 0.72 & 97.37 & 70.49 & 21.52 & 78.40 & 0.08 & \end{array}$

${ }^{a}$ Polarisation beider Bindungspartner bzw. Anteile an der Bindung

${ }^{b}$ Hierbei wurden nur Anteile über $1 \%$ berücksichtigt.

${ }^{c}$ Bindungsindex nach Wiberg 
Tabelle 2.5: Ausgewählte Bindungsparameter für $\left[\mathrm{WO}(\mathrm{pdt})_{2}\right]^{z-}, \mathrm{z}=-2,-1,0$

\begin{tabular}{|c|c|c|c|c|c|c|c|c|c|c|}
\hline \multirow[t]{2}{*}{$A-B$} & \multirow[t]{2}{*}{ Typ } & \multirow[t]{2}{*}{$\% \mathrm{~A}$} & \multicolumn{3}{|c|}{ Hybridisierung } & \multirow[t]{2}{*}{$\% \mathrm{~B}$} & \multicolumn{3}{|c|}{ Hybridisierung } & \multirow[t]{2}{*}{ WBI } \\
\hline & & & s & $\mathrm{p}$ & d & & $\mathrm{s}$ & $\mathrm{p}$ & d & \\
\hline \multicolumn{11}{|c|}{$\left[\mathrm{W}^{I V} \mathrm{O}(\mathrm{pdt})_{2}\right]^{2-}$} \\
\hline $\mathrm{W}-\mathrm{S}(2)$ & $\sigma$ & 20.26 & 18.92 & 0.60 & 80.47 & 77.16 & 23.81 & 76.08 & 0.11 & 0.70 \\
\hline $\mathrm{W}-\mathrm{S}(3)$ & $\sigma$ & 19.99 & 18.11 & 0.64 & 81.24 & 77.18 & 23.73 & 76.15 & 0.11 & 0.71 \\
\hline $\mathrm{W}-\mathrm{S}(4)$ & $\sigma$ & 19.53 & 18.37 & 0.65 & 80.98 & 77.50 & 23.86 & 76.03 & 0.11 & 0.70 \\
\hline $\mathrm{W}-\mathrm{S}(5)$ & $\sigma$ & 20.750 & 18.84 & 0.60 & 80.57 & 76.81 & 23.65 & 76.24 & 0.11 & 0.71 \\
\hline $\mathrm{W}-\mathrm{O}(17)$ & $\sigma$ & 23.25 & 5.36 & 1.04 & 93.60 & 76.46 & 28.88 & 71.05 & 0.07 & \\
\hline $\mathrm{W}-\mathrm{O}(17)$ & $\pi$ & 22.75 & 0.00 & 0.40 & 99.60 & 76.71 & 0.00 & 99.95 & 0.05 & 1.72 \\
\hline $\mathrm{W}-\mathrm{O}(17)$ & $\pi$ & 23.32 & 0.00 & 0.39 & 99.61 & 76.08 & 0.00 & 99.95 & 0.05 & \\
\hline \multicolumn{11}{|c|}{$\left[\mathrm{W}^{V} \mathrm{O}(\mathrm{pdt})_{2}\right]^{2-}$} \\
\hline $\mathrm{W}-\mathrm{S}(2)$ & $\sigma$ & 22.28 & 16.34 & 1.03 & 82.63 & 74.55 & 20.13 & 79.73 & 0.13 & 0.75 \\
\hline $\mathrm{W}-\mathrm{S}(3)$ & $\sigma$ & 21.98 & 17.44 & 1.15 & 81.41 & 74.72 & 20.14 & 79.72 & 0.14 & 0.76 \\
\hline $\mathrm{W}-\mathrm{S}(4)$ & $\sigma$ & 24.08 & 18.94 & 0.87 & 80.20 & 73.14 & 19.12 & 80.71 & 0.17 & 0.75 \\
\hline $\mathrm{W}-\mathrm{S}(5)$ & $\sigma$ & 24.10 & 19.93 & 0.96 & 79.12 & 73.08 & 19.06 & 80.77 & 0.17 & 0.76 \\
\hline $\mathrm{W}-\mathrm{O}(17)$ & $\sigma$ & 24.18 & 5.17 & 0.93 & 93.90 & 75.51 & 26.75 & 73.17 & 0.08 & \\
\hline $\mathrm{W}-\mathrm{O}(17)$ & $\pi$ & 24.08 & 0.00 & 0.25 & 99.75 & 75.23 & 0.02 & 99.92 & 0.06 & 1.71 \\
\hline $\mathrm{W}-\mathrm{O}(17)$ & $\pi$ & 25.37 & 0.00 & 0.25 & 99.75 & 73.87 & 0.00 & 99.94 & 0.06 & \\
\hline \multicolumn{11}{|c|}{$\left[\mathrm{W}^{V I} \mathrm{O}(\mathrm{pdt})_{2}\right]^{2-}$} \\
\hline \multirow[t]{2}{*}{$\mathrm{W}-\mathrm{S}(2)$} & $\sigma$ & 22.93 & 16.21 & 1.06 & 82.74 & 73.80 & 18.40 & 81.43 & 0.17 & 0.87 \\
\hline & $\pi$ & 24.63 & 0.17 & 0.37 & 99.45 & 68.23 & 0.31 & 99.61 & 0.08 & \\
\hline $\mathrm{W}-\mathrm{S}(3)$ & $\sigma$ & 23.23 & 18.57 & 1.12 & 80.31 & 73.54 & 18.46 & 81.37 & 0.17 & 0.87 \\
\hline $\mathrm{W}-\mathrm{S}(4)$ & $\sigma$ & 25.77 & 18.33 & 0.79 & 80.88 & 70.36 & 16.17 & 83.62 & 0.21 & 0.87 \\
\hline \multirow[t]{2}{*}{$\mathrm{W}-\mathrm{S}(5)$} & $\sigma$ & 25.30 & 17.34 & 1.27 & 81.39 & 71.79 & 17.45 & 82.33 & 0.21 & 0.88 \\
\hline & $\pi$ & 15.94 & 1.60 & 0.77 & 97.63 & 75.64 & 0.16 & 99.76 & 0.08 & \\
\hline $\mathrm{W}-\mathrm{O}(17)$ & $\sigma$ & 23.83 & 5.36 & 0.79 & 93.85 & 75.63 & 26.42 & 73.49 & 0.08 & \\
\hline $\mathrm{W}-\mathrm{O}(17)$ & $\pi$ & 25.93 & 0.01 & 0.14 & 99.85 & 73.24 & 0.00 & 99.93 & 0.07 & 1.76 \\
\hline $\mathrm{W}-\mathrm{O}(17)$ & $\pi$ & 26.03 & 0.01 & 0.16 & 99.83 & 73.14 & 0.00 & 99.93 & 0.07 & \\
\hline
\end{tabular}




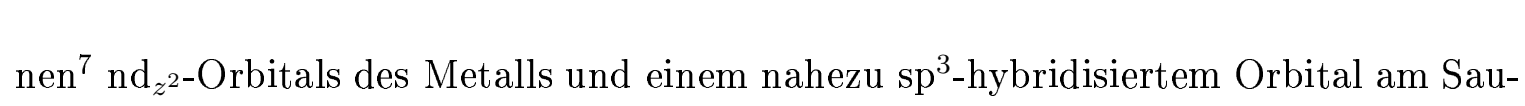
erstoff. Die beiden $\pi$-Bindungen resultieren dagegen aus einer Überlappung reiner d-Orbitale des Metalls mit reinen p-Orbitalen des Sauerstoffs. Die Bindung ist stark polarisiert, was sich auch an den Werten von 1.7 bis 1.8 des WBI widerspiegelt. Die Polarisierung in Richtung des Sauerstoff ist bei den Wolframverbindungen stärker ausgeprägt als bei den Molybdänverbindungen. Bei beiden erfolgt eine Zunahme beim Wechsel von Oxidationsstufe VI zu V und eine marginale Zunahme beim Wechsel von V zu IV. Der sich dabei verändernde Anteil wird im Fall der Molybdänverbindungen durch die $\sigma$-Bindung verursacht, deren Anteil an der Bindung von 29.2\% (OS VI) auf $26.4 \%$ absinkt; im Fall der Wolframverbindungenjedoch von den $\pi$-Bindungen, deren Anteile von $26.0 \%$ auf $22.8 / 23.3 \%$ sinken.

Die vier das Metall umgebenden Schwefel-Atome sind kovalent über $\sigma$-Bindungen gebunden. Die Anteile an der Bindung (ca. 25\% Metall und 75\% Schwefel) sowie die Polarisierung ähneln der Metall-Sauerstoff-Bindung. Im Gegensatz dazu findet allerdings beim Wechsel der Oxidationsstufe eine Änderung im Bindungsverhalten statt. Abbildung 2.6 zeigt als Lewisstrukturen dargestellt das unterschiedliche Verhalten der Bindungen.

Sowohl beim Molybdän als auch beim Wolfram in den Oxidationsstufen IV und V ist jeder Schwefel über eine Einfachbindung, bestehend aus der Überlappung eines $\mathrm{sp}^{3}$-hybridisierten Schwefel-Atoms und einem sd-Hybrid des Metalls an das Metall gebunden und verfügt über zwei freie Elektronenpaare. Diese bestehen jeweils aus einem sp-Hybrid und einem reinen p-Orbital. Beim Wechsel in die Oxidationsstufe VI des Metalls wird formal eines der p-Orbitale zur Bindung an das Metall verwendet, bei der Molybdänverbindung einmal und bei der Wolframverbindung sogar zweimal. Dies geschieht offensichtlich zur Absättigung der elektronischen Dichte am Metall,

\footnotetext{
${ }^{7}$ s-Anteile ca. $2 \%$
} 
Abbildung 2.6: Lewisstrukturen der Komplexe in den verschiedenen OS

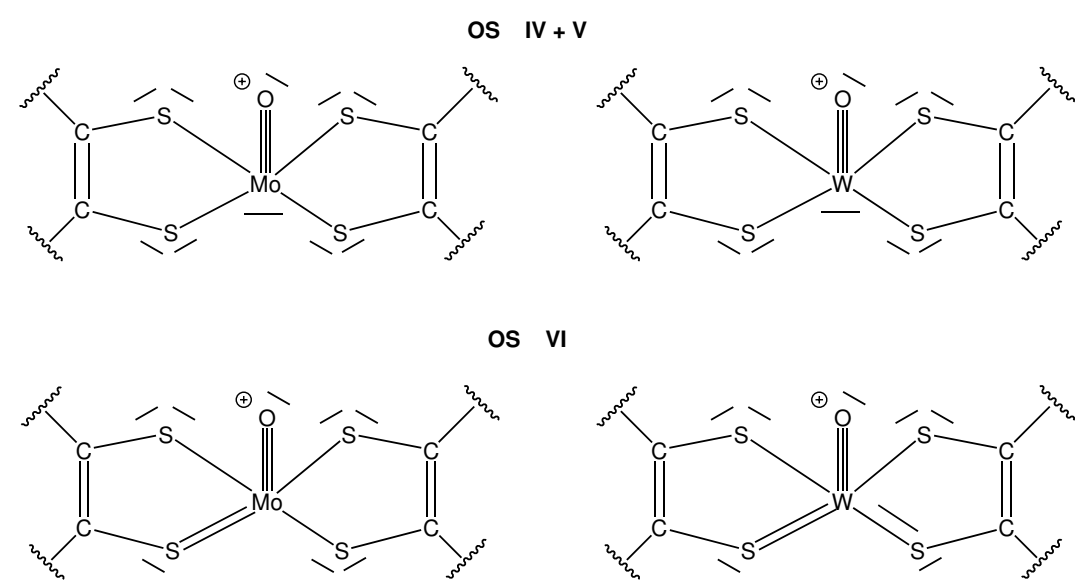

die beim Entfernen der zwei Elektronen durch den Oxidationsprozess dem Metall entzogen werden welches auch durch das Verschwinden des freien Elektronenpaars am Metall selbst sichtbar wird. Hierdurch wird eine wesentlich stärkere Redoxaktivität von Wolfram gegenüber Molybdän deutlich, die von zwei Schwefel-Atomen "gepuffert" werden muss.

Eine Neigung zum Beteiligen der freien Elektronenpaare des Schwefels an der Metall-Schwefel-Bindung zeigt sich auch deutlich an den Werten der Zusammensetzung ihrer Anteile. Tabelle 2.6] zeigt als Beispiel die Zusammensetzung der freien Elektronenpaare am $\mathrm{S}(2)$ der Verbindung 1a. Die jeweiligen reinen p-Orbitale mischen in allen Fällen mit nahezu reinen d-Orbitalen des Metalls. Die Anteile reichen dabei von etwa 5\% Metallanteil in den Oxidationsstufen IV und V bis hin zu ca. $13 \%$ in Oxidationsstufe VI. Hierbei handelt es sich um die verbleibenden freien Elektronenpaare. Auch hier zeigt sich ein marginaler Trend zu Gunsten des Wolframs, dessen Beimischung etwas stärker als bei den Molybdänverbindungen ausfällt.

Unterschiede der Bindungen werden durch die Werte des WBI verdeutlicht: beim Wechsel der Oxidationsstufen steigt die Bindungsstärke um 0.05 Einheiten von IV zu V sowie um 0.1 Einheiten beim Wechsel von V zu VI. Dabei ist der Bindungsindex 
Tabelle 2.6: Bindungsparameter der freien Elektronenpaare von Mo(1) und S(2) der Verbindung $\left[\mathrm{Mo}^{I V} \mathrm{O}(\mathrm{pdt})_{2}\right]^{2-}$

\begin{tabular}{lcccclccccc}
\hline $\mathrm{A}^{a}$ & \% A & \multicolumn{3}{c}{ Hybridisierung } & $\mathrm{B}^{a}$ & \multicolumn{3}{c}{ \% B } & \multicolumn{3}{c}{ Hybridisierung } \\
& & $\mathrm{s}$ & $\mathrm{p}$ & $\mathrm{d}$ & & & $\mathrm{s}$ & $\mathrm{p}$ & $\mathrm{d}$ \\
\hline $\mathrm{LP}{ }^{b} \mathrm{Mo}(1)$ & 95.49 & 0.04 & 0.03 & 99.92 & & & & & \\
$\mathrm{LP}(1) \mathrm{S}(2)$ & 98.76 & 62.77 & 37.22 & 0.02 & & & & & \\
$\mathrm{LP}(2) \mathrm{S}(2)$ & 87.68 & 0.02 & 99.95 & 0.03 & $\mathrm{Mo}(1)$ & 4.38 & 1.07 & 0.88 & 98.05 \\
& & & & & $\mathrm{C}(6)$ & 2.91 & 0.01 & 98.39 & 1.60 \\
& & & & & $\mathrm{C}(7)$ & 2.48 & 0.33 & 99.56 & 0.10 \\
& & & & & $\mathrm{O}(17)$ & $<2$ & & &
\end{tabular}

${ }^{a}$ A, B sind an der Wechselwirkung beteiligte Atome

${ }^{b}$ LP steht für "lone pair"

der Wolframverbindungen jeweils um ca 0.5 Einheiten größer als bei den Molybdänverbindungen; bei der OS VI ist der Unterschied mit ca. 0.7 noch etwas größer. Diese Werte verdeutlichen eine zunehmende Stärkung der Metall-Schwefel-Bindungen bei höheren Oxidationszahlen beim Wolfram gegenüber dem Molybdän, was sich auch in der Verkürzung der Bindungslängen widerspiegelt.

Die Schwefel-Kohlenstoffbindungen der Dithioleneinheit sind reine $\sigma$-Einfachbindungen und resultieren aus der Überlappung von $\mathrm{sp}^{3}$-Hybridorbitalen des Kohlenstoffs und Schwefels. Die Bindungsanteile werden von beiden Atomen zu fast gleichen Anteilen gestellt. Im Verlauf der Oxidation findet hierbei keine Änderung statt. Lediglich der Bindungsindex steigt mit zunehmender Oxidationsstufe, bei Molybdän von 1.06 (IV) über $1.10(\mathrm{~V})$ bis hin zu 1.2; die Werte für die Wolframverbindungen sind von ähnlicher Größe jedoch liegen sie bei OS VI mit 1.15 etwas unter den Werten für Molybdän (gemittelte Werte). Interessanterweise fallen die Werte für die Bindungen auf der Seite des Faltungswinkels niedriger aus. Betrachtet man die Werte der C-C-Doppelbindungen, welche in allen Fällen aus je einer $\sigma$ - und einer $\pi$-Bindung besteht, so zeigt sich eine Abnahme des BDIs von 1.67 über 1.65 bis auf 1.51 (Molybdän) bzw. von 1.69 über 1.67 bis hin zu 1.56 für die Wolframverbindungen. D.h. die 
C-S-Bindungen werden mit zunehmender OS stärker und zwar bei den Wolframverbindungen in höherem Maße; die C-C-Doppelbindungen dagegen werden schwächer und zwar bei den Molybdänverbindungen in höherem Maße.

Zusammenfassend lässt sich aussagen, dass bei Abzug von Elektronen aus dem Molekül die direkt das Metall betreffenden Bindungen kürzer werden um die verbleibende Elektronendichte besser nutzen zu können, wobei Elektronen aus dem $\pi$-Rückgrat der C-C-Doppelbindung mit einbezogen werden. Verdeutlicht wird dies am Verhalten der Ausbildung der formalen p- $\pi$-d- $\pi$-Doppelbindungen, bei denen auf die Elektronendichte der freien Elektronenpaare des Schwefels zurückgegriffen wird um das Metall abzusättigen.

Somit fungiert der Dithiolenligand als variabler $\pi$-Donator. Verstärkt wird diese Eigenschaft durch die leicht verfügbaren $\pi$-Elektronen der C-C-Doppelbindung und die Möglichkeit, Elektronendichte innerhalb der freien Elektronenpaare je nach Bedarf abzugeben oder aufzunehmen. Weiterhin spielt auch der Einfluss des Sauerstoff-Atoms eine große Rolle, wobei hier der erste Schritt der Oxidation (IV zu V) stärkere Auswirkungen auf Änderungen im Bindungsverhalten hat als der zweite Schritt, welcher eher Auswirkungen auf die Dithiolenliganden hat.

Der Einfluss des axialen Sauerstoff-Atoms zeigt sich vor allem in der Aufspaltung der Orbitale. Die gezeigte Aufspaltung resultiert einerseits aus dem Einfluss des Sauerstoffs, andererseits aus dem schwachen Ligandenfeld der restlichen Liganden. Eine starke Destabilisierung findet bei den redoxaktiven $\mathrm{d}_{x^{2} y^{2}}$-Orbitalen durch antibindende Wechselwirkungen mit dem Sauerstoff statt [121], so dass diese energetisch so weit angehoben sind, dass sie unbesetzt bleiben. Tabelle 2.7 zeigt die Orbitalenergien der Orbitale 86 bis 91, darunter das HOMO (88) und das LUMO (89) der entsprechenden Pyrankomplexe sowie den Wert für die HOMO-LUMO-Lücke.

Auffallend ist, dass die Orbitalenergien der Molybdän- und Wolfram-Komplexe 
Tabelle 2.7: Orbitalenergien $\varepsilon$ der Orbitale 86 - 90 [Hartree]

\begin{tabular}{|c|c|c|c|c|c|c|c|}
\hline & Orbital & $\mathbf{1 a}$ & $2 a$ & $3 \mathbf{a}$ & $4 a$ & $5 \mathbf{a}$ & $6 \mathbf{6 a}$ \\
\hline & 86 & 0.05 & -0.12 & -0.25 & 0.04 & -0.11 & -0.25 \\
\hline & 87 & 0.07 & -0.09 & -0.23 & 0.07 & -0.1 & -0.23 \\
\hline $\mathrm{HOMO}$ & 88 & 0.07 & -0.07 & -0.16 & 0.08 & -0.07 & -0.15 \\
\hline \multirow[t]{3}{*}{ LUMO } & 89 & 0.22 & 0.06 & -0.08 & 0.22 & 0.08 & -0.07 \\
\hline & 90 & 0.22 & 0.06 & -0.08 & 0.23 & 0.09 & -0.06 \\
\hline & 91 & 0.23 & 0.08 & -0.06 & 0.24 & 0.1 & -0.05 \\
\hline$\Delta \varepsilon(89-88)$ & & 0.15 & 0.13 & 0.08 & 0.14 & 0.15 & 0.08 \\
\hline
\end{tabular}

wesentlich größer sind als die der vergleichbaren Nickelkomplexe [122]. So betragen die Energien für das $\mathrm{HOMO}$ von $\mathrm{Ni}\left(\mathrm{S}_{2} \mathrm{C}_{2} \mathrm{Me}_{2}\right)_{2}$ sowie $\mathrm{Ni}(\mathrm{dmit})_{2}$ je -0.22 Hartree. Der enorme Unterschied zwischen den Nickel- und Molybdän-/Wolfram-Komplexen ist dabei auf die oben erwähnte destabilisierende Wirkung des axialen SauerstoffLiganden zurückzuführen.

Beim Wechsel des Metalls von Molybdän zu Wolfram findet sich wiederum kein großer Unterschied, lediglich ist bei den Molybdän-Komplexen ein Absinken der HOMOLUMO-Lücke von 0.15 zu 0.13 atomaren Einheiten bei dem Schritt von OS IV zu V zu beobachten, während sich bei den Wolfram-Komplexen ein leichter Anstieg von 0.14 auf 0.15 atomaren Einheiten zeigt. Die HOMO-LUMO-Lücke in der OS VI wird durch das Entfernen der beiden Elektronen zur LUMO+1-LUMO-Lücke und ist wiederum nur noch halb so groß wie in den niedrigeren OS. Das in den OS IV und V am höchsten besetzte $\mathrm{d}_{x^{2} y^{2}}$-Orbital (OS V SOMO) verbleibt in OS VI nach Entfernen der beiden Elektronen unbesetzt und wird dadurch energetisch abgesenkt. Das in OS VI höchste besetzte Orbital ist nun ein an den Schwefel-Atomen konzentriertes outof-plane- $\pi$-Orbital, welches keine Wechselwirkung mit dem axialen Sauerstoff-Atom aufweist. Die Gestalt und Zusammensetzung der betreffenden Orbitale ist in Tabelle 2.8 gezeigt, Abbildung 2.7 enthält die beiden Grenzorbitale von 1a und $\mathbf{2 a}$ im 
Tabelle 2.8: Zusammensetzung der redoxaktiven Orbitale (88)

\begin{tabular}{cccccc}
\hline & $(\mathrm{Mo}, \mathrm{W})\left(\mathrm{d}_{x^{2} y^{2}}\right)$ & $\mathrm{S}(2)\left(\mathrm{p}_{z}\right)$ & $\mathrm{S}(3)\left(\mathrm{p}_{z}\right)$ & $\mathrm{S}(4)\left(\mathrm{p}_{z}\right)$ & $\mathrm{S}(5)\left(\mathrm{p}_{z}\right)$ \\
\hline $\mathbf{1 a}$ & 0.39793 & -0.10014 & -0.10005 & 0.09122 & 0.09109 \\
$\mathbf{1 b}$ & 0.31946 & -0.10622 & -0.10574 & 0.09529 & 0.09406 \\
$\mathbf{1 c}$ & 0.50632 & -0.09187 & -0.09512 & 0.07790 & 0.08150 \\
$\mathbf{2 a}$ & 0.74354 & $-0.02250\left(\mathrm{p}_{y}\right)$ & 0.02358 & 0.02317 & -0.02283 \\
$\mathbf{2 b}$ & 0.39038 & -0.10385 & -0.10291 & 0.09136 & 0.09037 \\
$\mathbf{2 c}$ & 0.57826 & -0.08305 & -0.08744 & 0.06969 & 0.07430 \\
& & & & & \\
\hline & $\mathrm{C}(6)\left(\mathrm{p}_{z}\right)$ & $\mathrm{C}(7)\left(\mathrm{p}_{z}\right)$ & $\mathrm{C}(12)\left(\mathrm{p}_{z}\right)$ & $\mathrm{C}(13)\left(\mathrm{p}_{z}\right)$ & $\mathrm{O}(17)\left(\mathrm{p}_{x}\right)$ \\
\hline & & & & & \\
$\mathbf{1 a}$ & 0.06471 & 0.06249 & -0.07169 & -0.07375 & -0.05473 \\
$\mathbf{1 b}$ & 0.07180 & 0.07056 & -0.08011 & -0.08117 & -0.06208 \\
$\mathbf{1 c}$ & 0.04202 & 0.04207 & -0.07286 & -0.07261 & -0.04443 \\
$\mathbf{2 a}$ & $0.03157\left(\mathrm{p}_{y}\right)$ & -0.03026 & -0.03044 & 0.03115 & $<0.001$ \\
$\mathbf{2 b}$ & 0.07018 & 0.06838 & -0.07805 & -0.07626 & -0.05944 \\
$\mathbf{2 c}$ & 0.04298 & 0.04570 & -0.06658 & -0.06776 & -0.04116
\end{tabular}

Abbildung 2.7: Grenzorbitale von $\left[\mathrm{M}^{I V} \mathrm{O}(\mathrm{pdt})_{2}\right]^{2-}, \mathrm{M}=\mathrm{Mo}, \mathrm{W}$ im Vergleich

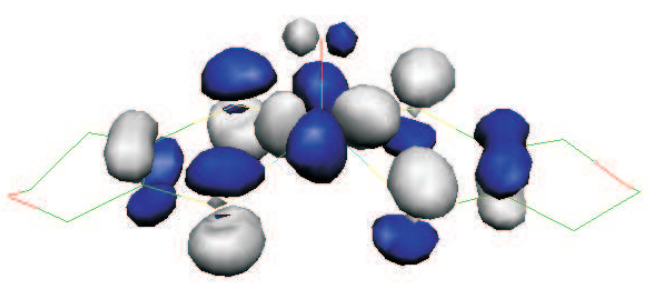

HOMO 1a

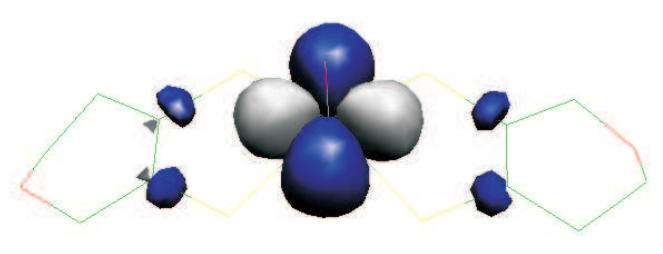

HOMO 2a

Vergleich. Die Zusammensetzung ist anhand der Orbitalkoeffizienten gegeben, die näherungsweise als prozentuale Anteile verwendet werden.

Das HOMO von 1a (Mo, OS IV) besteht aus dem $\mathrm{d}_{x^{2} y^{2}}$-Orbital des Metalls sowie $\mathrm{p}_{z}$-Orbitalen des Schwefels und der zur Dithioleneinheit gehörenden KohlenstoffAtome der C-C-Doppelbindung sowie einem $\mathrm{p}_{y}$-Orbital des axialen Sauerstoff-Liganden. Diese charakteristische Zusammensetzung bleibt bei allen OS erhalten wobei sich jedoch die räumliche Ausrichtung und die Zusammensetzung der einzelnen Hybridorbitale verändern. 
Die Grenzorbitale der analogen Wolfram-Verbindungen unterscheiden sich lediglich in OS IV sowie in den einzelnen Beiträgen der Hybridorbitale. Der Wolframkomplex in OS IV ist mit einem hohen metallischen Anteil von $74 \%$ nur wenig von den Liganden kontrolliert. Hierbei mischen die Schwefel-Atome im Gegensatz zu den anderen Verbindungen über ihre y-Komponente ("in-plane") und betragen anteilsmäßig nur $2 \%$. Der axiale Sauerstoff trägt mit nur $1 \%$ zum Orbital bei und zwar mit dem $\mathrm{p}_{z^{-}}$ Orbital. Der entsprechende Molybdän-Komplex verfügt nur über einen metallischen Anteil von 40\%, die Anteile der Schwefel- $\mathrm{p}_{z}$-Orbitale betragen dagegen durchschnittlich $10 \%$ pro Atom. Beim Wechsel in die OS V wird eine Abnahme des metallischen Charakters beobachtet, beim Mo von 40 auf $32 \%$ und beim W von 74 zu 39\%. Die Anteile der Schwefel-Beiträge steigen dagegen nur marginal um 1-2\% (Mo), wobei der Wolframkomplex nun ähnlich dem Mo-Komplex $\mathrm{p}_{z}$-Orbital-Anteile der SchwefelAtome aufweist. Beim Wechsel in die OS VI ist bei beiden Metallen ein Anstieg des metallischen Charakters (Mo 51\%, W 58\%) zu verzeichnen, der Anteil der SchwefelOrbitale sinkt dagegen marginal. Die Zusammensetzung des HOMOs in OS VI ist im wesentlichen von p-Beiträgen der Schwefel-Atome geprägt. Zwischen den Metallen Mo und $\mathrm{W}$ zeigt sich wiederum ein sehr geringer Unterschied zugunsten des Wolframs. Dagegen beobachteten Holm et al. eine gleichmäßige Abnahme des Metall- und eine Zunahme des Schwefel-Charakters mit zunehmender Oxidation [122]. Daraus wurde geschlussfolgert, dass die Oxidation essentiell ligandenbasiert abläuft und vor allem bei Wechsel von OS IV zu V die Dithiolenliganden in der Lage sind, gewissermaßen Elektronen(-dichte) zu "absorbieren". Dieser Trend während des Oxidationsprozesses ist dagegen bei den Oxokomplexen kaum zu erkennen. Der Metallcharakter beim Wechsel von OS IV zu V nimmt zwar deutlich ab, jedoch erfolgt in OS VI wiederum eine enorme Zunahme des Metallcharakters.

Dieser Unterschied verdeutlicht einerseits den Einfluss des axialen Sauerstoffs, der 
sich auch im Auftreten des sogenannten Faltungswinkels äußert8, der mit für dieses Phänomen verantwortlich ist, andererseits auch den Einfluss durch das Vorhandensein weiterer Atome im Rückgrat der Dithiolengruppe. Die von Holm et al. behandelten Systeme verfügen lediglich über je zwei an die Dithiolengruppe geknüpfte Methylgruppen, so dass sämtliche Elektronendichte der Orbitale zwischen dem Metall und der Dithioleneinheit aufgeteilt wird. Im Fall der Pyran-Liganden wird ein Teil der Elektronendichte in das Rückgrat weitergeleitet, wenn auch in kleinen Anteilen pro Atom, wie sich an den geringen Schwankungen der absoluten Werte der Orbitalkoeffizienten zeigt.

Das Phänomen des Faltungswinkels und sein Auftreten in Zusammenhang mit der Besetzung von Metallorbitalen in Dithiolenkomplexen wurde von Fourmigue et al. ausführlich an Verbindungen der Art $\mathrm{Cp}_{2} \mathrm{M}$ (dithiolen) ( $\mathrm{M}=\mathrm{Ti}, \mathrm{Zr}, \mathrm{Hf}, \mathrm{V}, \mathrm{Nb}, \mathrm{Mo}$, W) untersucht [123].

Der von dem Metallocyclus entlang des S-S-Vektors liegende Winkel ändert sich in Abhängigkeit der Besetzung der d-Orbitale des Metalls. So haben Verbindungen mit der formalen $\mathrm{d}^{0}$-Besetzung die größten Faltungswinkel während er bei $\mathrm{d}^{2}$ Verbindungen gegen $0^{\circ}$ tendiert. Das "Abknicken" eines Liganden erfolgt zwecks einer Stabilisierung durch die so vermehrte Wechselwirkung und Überlappungsmöglichkeit der besetzten out-of-plane-p-Orbitale des Schwefels mit dem in-plane-d-Orbital des Metalls. Die vom axialen Sauerstoff hervorgerufene Geometrieänderung von quadratischplanar zu quadratisch-pyramidal vermindert durch das Anheben des Metalls über die Schwefel-Ebene 9 die Überlappung der Schwefel-Atome mit dem Metall, so dass durch das "Falten" eines Liganden eine zusätzliche Überlappung und damit verbunden eine Stabilisierung erreicht wird.

Die Oxidationsstufe des Metalls bzw. die Zahl der im $\mathrm{d}_{x^{2} y^{2}}$-Orbital vorhandenen

\footnotetext{
${ }^{8}$ im Falle der Nickelserie nicht beobachtet

${ }^{9}$ Gemeint ist die von den vier Schwefelatomen aufgespannte Ebenen $\mathrm{S}(2)-\mathrm{S}(3)-\mathrm{S}(4)-\mathrm{S}(5)$
} 
Elektronen haben folgenden Einfluss auf die Geometrie. In der Oxidationsstufe IV (formal $\mathrm{d}^{2}$ ) liegen die Verbindungen ähnlich den quadratisch-planaren Komplexen in $\mathrm{c}_{2 v}$-Symmetrie vo1 10 . Durch die schon im d-Orbital des Metalls vorhandenen Elektronen ist eine $\pi$-Donation nicht von Nöten und würde eher zu einer Abstoßung der Orbitale führen, somit verbleibt die Konfiguration in $\mathrm{c}_{2} \mathrm{v}-\mathrm{Symmetrie}$. Im Fall der OS V und VI $\left(\mathrm{d}^{1}\right.$ und $\left.\mathrm{d}^{0}\right)$ wird eine graduelle Stabilisierung durch das Falten eines Liganden und die entsprechende $\pi$-Donation ermöglicht. Das Abknicken beider Liganden jedoch würde zu einer Situation führen, in der es zu einer Wechselwirkung kommt, welche daraus resultiert, dass in der sich ergebenden twist-Konfiguration die Stabilisierung einer Seite zwangsläufig einhergeht mit einer gleichzeitigen Destabilisierung der anderen Seite. Abbildung 2.8 zeigt die jeweiligen Grenzorbitale (LUMO in OS VI) mit Schnitten zur besseren Verdeutlichung der betreffenden Überlappungen.

Deutlich erkennbar ist die nachlassende Überlappung der Schwefel-Atome mit dem Metall auf der Seite des nicht abknickenden Liganden. Dies erklärt die graduelle Abstufung der Veränderung des Faltungswinkels, welcher beim Wechsel von OS IV zu V wesentlich und beim Wechsel von V zu VI geringer ausfällt. Die in OS V erreichte Überlappung gewährleistet auch unter geringerer Zunahme des Faltungswinkel in OS VI eine erfolgreiche $\pi$-Donation unter Berücksichtigung der destabilisierenden Wechselwirkung.

Von wesentlichem Interesse ist weiterhin der ersichtliche Unterschied beider Metalle, Mo und W. Wie bereits oben diskutiert, ist der metallische Anteil an den Grenzorbitalen in den Wolfram-Spezies in allen Fällen höher, in OS IV sogar fast doppelt so hoch wie beim Molybdän. Auch die Gestalt des sp-Orbitals ändert sich beim Wechsel des Metalls. So beteiligt sich beim Wolfram das in-plane- $\mathrm{p}_{y}$-Orbital des Schwefels statt des $\mathrm{p}_{z}$-Orbitals. Die relative energetische Lage sowohl der 3p Orbitale des Schwe-

\footnotetext{
${ }^{10} c_{\mathrm{s}}$-Symmetrie wegen transständiger Pyransauerstoffe
} 
Abbildung 2.8: Schnitte durch die redoxaktiven Orbitale 1a, 1b, 1c

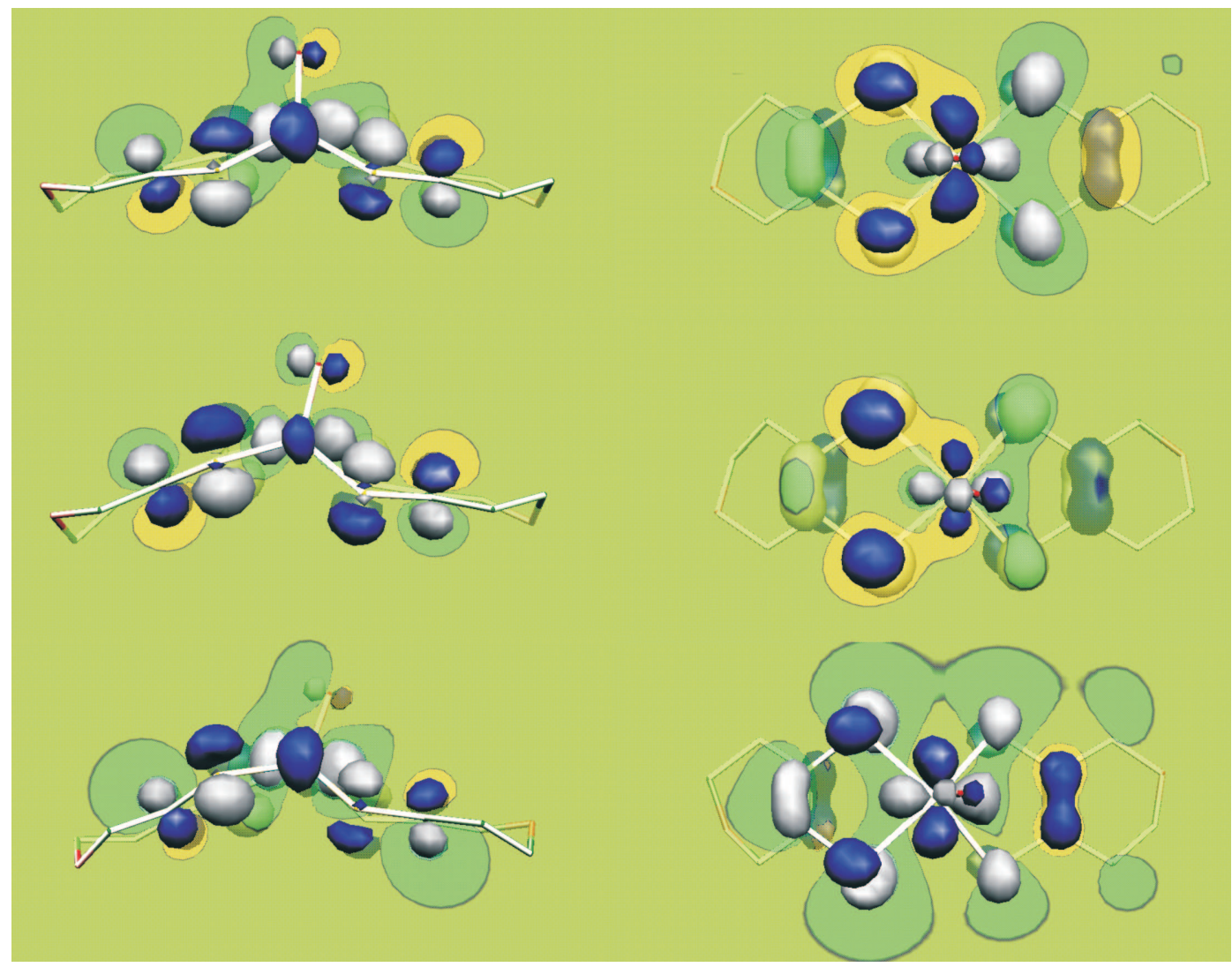

fels als auch der 4d/5d-Orbitale des Metalls resultiert aus der effektiven Kernladung $\left(\mathrm{Z}_{e f f}\right)$ und skalaren relativistischen Effekten des Metalls [124]. Die sich nicht abzeichnende Orbitalinversion des Schwefels in den OS V und VI zeigt somit deutlich die Bevorzugung hoher OS des Wolframs. Während es in diesen OS möglich ist, die Wolframverbindungen über die $\pi$-Donation mit Hilfe des Faltungswinkels zu stabilisieren ist dies in OS IV nicht möglich. Der hohe metallische Anteil von $74 \%$ in OS IV zeigt das Ausmaß der zunehmenden effektiven Kernladung und der relativistischen Effekte. Die im Falle der Molybdänverbindung noch ausreichende schwache Überlappung der out-of-plane- $\mathrm{p}_{z}$-Orbitale reicht beim Wolfram nicht aus, um das Maß an erforderlicher Elektronendichte zu decken, so dass hier ein in-plane- $\mathrm{p}_{y}$-Orbital herangezogen wird, um über eine bessere Überlappung die elektronische Dichte am Metall zu ge- 
währleisten. In den OS V und VI wiederum ist über die Faltung gewährleistet, dem Metall ausreichend Elektronendichte zukommen zu lassen, so dass in diesen Fällen die $\mathrm{p}_{z}$-Orbitale genügen.

Ein weiterer Aspekt der Dithiolenbindung ist die von Inscore et al. aufgestellte oxo-gate-Hypothese. Spektroskopische Studien zeigen eine hohe kovalente Wechselwirkung zwischen dem redoxaktiven Orbital am Metall und den Orbitalen am Schwefel [125, 126]. Dabei überlappt das $\mathrm{d}_{x^{2} y^{2}}$-Orbital des Metalls direkt mit den in-plane- $\mathrm{p}_{x^{-}}$ Orbitalen des Schwefels sowie diese mit sich selbst und es resultiert eine DreizentrenPseudo- $\sigma$-Bindung zwischen dem Metall und jeweils zwei Schwefel-Atomen eines Dithiolenliganden. Diese Überlappung ist nur durch eine orthogonale Stellung des axialen Sauerstoff-Atoms bezüglich der Metall-Schwefel-Bindung möglich. Eine nahezu orthogonale Stellung wird in allen drei OS bezüglich einer Seite des Liganden beobachtet. Die $\sigma$-Bindung ermöglicht somit einen elektronischen Weg in das Rückgrat des Liganden, welcher im Falle des Pyranopterins über keine ausgedehnten $\pi$-Elektronensysteme verfügt.

Um die Wechselwirkungen zwischen jeweils zwei benachbarten Schwefel-Atomen in unseren Verbindungen zu untersuchen, wurden diesbezüglich NBO-Analysen der betreffenden Atome durchgeführt. Tabelle 2.9 zeigt die jeweiligen Anteile, Besetzungen und Orbitalzusammensetzungen der entsprechenden Bindungspartner.

Wechselwirkungen die zu einer Bindung führen sind nur in OS IV vorhanden. In OS V gibt es keinerlei Wechselwirkungen zwischen den Schwefel-Atomen und in OS VI überwiegt der antibindende Anteil.

Beim Mo IV-Komplex sind bei beiden Dithiolenliganden ähnliche Verhältnisse zu finden; zwei $\mathrm{sp}^{3}$-Hybridorbitale überlappen mit bindenden und antibindenden Anteilen (Abbildung 2.9), wobei der bindende Anteil dominiert und eine Wechselwirkung vorhanden ist. Beide Schwefel-Atome haben jeweils den gleichen Anteil an der 
Tabelle 2.9: Bindunsparameter der benachbarten S-Atome

\begin{tabular}{|c|c|c|c|c|c|c|c|c|c|c|}
\hline \multirow[t]{2}{*}{$\overline{A-B}$} & \multirow[t]{2}{*}{ Besetzung } & \multirow[t]{2}{*}{$\% \mathrm{~A}$} & \multicolumn{3}{|c|}{ Hybridisierung } & \multirow[t]{2}{*}{$\% \mathrm{~B}$} & \multicolumn{3}{|c|}{ Hybridisierung } & \multirow[t]{2}{*}{$\mathrm{BO}^{a}$} \\
\hline & & & $\mathrm{s}$ & $\mathrm{p}$ & d & & $\mathrm{s}$ & $\mathrm{p}$ & d & \\
\hline \multicolumn{11}{|c|}{$\left[\mathrm{Mo}^{I V} \mathrm{O}(\mathrm{pdt})_{2}\right]^{2-}$} \\
\hline $\mathrm{S}(2)-\mathrm{S}(3)$ & 1.71 & 50.59 & 21.98 & 77.92 & 0.10 & 49.41 & 22.59 & 77.30 & 0.10 & 0.14 \\
\hline $\mathrm{S}(2)-\mathrm{S}(3) * b$ & 1.43 & 49.41 & 21.98 & 77.92 & 0.10 & 50.59 & 22.59 & 77.30 & 0.10 & \\
\hline $\mathrm{S}(4)-\mathrm{S}(5)$ & 1.71 & 50.40 & 22.06 & 77.84 & 0.10 & 49.60 & 22.47 & 77.42 & 0.10 & 0.14 \\
\hline $\mathrm{S}(4)-\mathrm{S}(5) *$ & 1.43 & 49.60 & 22.06 & 77.84 & 0.10 & 50.40 & 22.47 & 77.42 & 0.10 & \\
\hline \multicolumn{11}{|c|}{$\left[\mathrm{Mo}^{V I} \mathrm{O}(\mathrm{pdt})_{2}\right]$} \\
\hline $\mathrm{S}(2)-\mathrm{S}(3)$ & 1.27 & 49.91 & 0.53 & 99.38 & 0.09 & 50.09 & 0.07 & 99.84 & 0.09 & $(-0.21)^{c}$ \\
\hline $\mathrm{S}(2)-\mathrm{S}(3) *$ & 1.69 & 50.09 & 0.53 & 99.38 & 0.09 & 49.91 & 0.07 & 99.84 & 0.09 & \\
\hline $\mathrm{S}(4)-\mathrm{S}(5)$ & 1.41 & 46.33 & 0.39 & 99.52 & 0.09 & 53.67 & 0.02 & 99.88 & 0.09 & $(-0.14)^{c}$ \\
\hline $\mathrm{S}(4)-\mathrm{S}(5) *$ & 1.6 & 53.67 & 0.39 & 99.52 & 0.09 & 46.33 & 0.02 & 99.88 & 0.09 & \\
\hline \multicolumn{11}{|c|}{$\left[\mathrm{WO}^{I V}(\mathrm{pdt})_{2}\right]^{2-}$} \\
\hline $\mathrm{S}(2)-\mathrm{S}(3)$ & 1.68 & 50.87 & 26.96 & 72.92 & 0.12 & 49.13 & 27.64 & 72.23 & 0.12 & 0.11 \\
\hline $\mathrm{S}(2)-\mathrm{S}(3)^{*}$ & 1.47 & 49.13 & 26.96 & 72.92 & 0.12 & 50.87 & 27.64 & 72.23 & 0.12 & \\
\hline $\mathrm{S}(4)-\mathrm{S}(5)$ & 1.75 & 0.16 & 10.71 & 48.15 & 41.14 & 99.84 & 0.01 & 99.96 & 0.03 & 0.88 \\
\hline $\mathrm{S}(4)-\mathrm{S}(5) *$ & - & - & - & - & - & - & - & - & - & \\
\hline \multicolumn{11}{|c|}{$\left[\mathrm{WO}^{V I}(\mathrm{pdt})_{2}\right]$} \\
\hline $\mathrm{S}(2)-\mathrm{S}(3)$ & 1.33 & 50.28 & 0.32 & 99.60 & 0.08 & 49.72 & 0.02 & 99.90 & 0.08 & $(-0.19)^{c}$ \\
\hline $\mathrm{S}(2)-\mathrm{S}(3) *$ & 1.70 & 49.72 & 0.32 & 99.60 & 0.08 & 50.28 & 0.02 & 99.90 & 0.08 & \\
\hline $\mathrm{S}(4)-\mathrm{S}(5)$ & 1.44 & 46.16 & 0.29 & 99.63 & 0.08 & 53.84 & 0.07 & 99.84 & 0.09 & $(-0.13)^{c}$ \\
\hline $\mathrm{S}(4)-\mathrm{S}(5) *$ & 1.71 & 53.84 & 0.29 & 99.63 & 0.08 & 46.16 & 0.07 & 99.84 & 0.09 & \\
\hline
\end{tabular}


Abbildung 2.9: Darstellung der bindenden und antibindenden Wechselwirkungen der benachbarten S-Atome
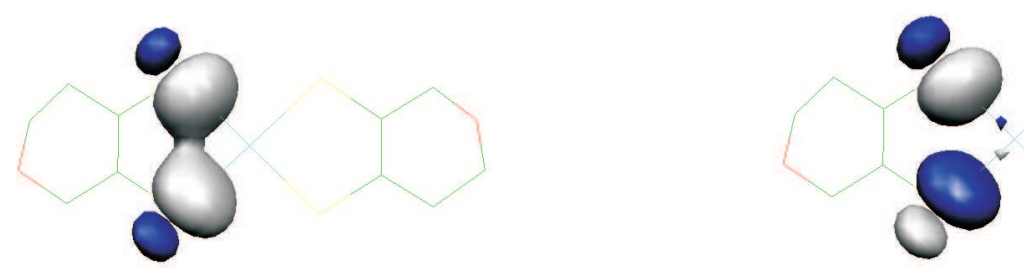

Bindung. Bei der W(IV)-Verbindung besteht eine S-S-Wechselwirkung ähnlich der Mo-Verbindung aus zwei $\mathrm{sp}^{3}$-Hybriden ähnlicher Größenordnung, Die andere S-SWechselwirkung jedoch besitzt in der Hybridisierung d-Anteile und besteht aus nur bindenden Anteilen. Die Werte der Bindungsanalyse zeigen, dass diese Art der Wechselwirkung ein nicht zu vernachlässigendes Strukturelement innerhalb von Oxo-Molybdän und -Wolfram-Dithiolenzentren ist, da damit ein weiterer Weg aufgewiesen wird, den Elektronentransfer zwischen Metall und Ligand zu unterstützen.

\subsection{Die Systeme $\left[\mathrm{MO}(\mathrm{cdt})_{2}\right]^{z-}$ und $\left[\mathrm{MO}(\mathrm{fdt})_{2}\right]^{z-}$}

\subsubsection{Synthese der Modellkomplexe $\mathrm{PPh}_{4}\left[\mathrm{MO}(\mathrm{cdt})_{2}\right]^{z-}(\mathrm{M}=\mathrm{Mo}$, W)}

Aufbauend auf die von Garner et al. [86] entwickelte Syntheseroute zur Darstellung der Modellkomplexe durch basische Umsetzung dithiocarbonatgeschützter Dithiolene analog dem in Abbildung 2.10 gezeigten Schema wurde der Ligand Chromanyldithiocarbonat 17 synthetisiert.

Das Ausgangsprodukt, Chromanon ist ein an Benzol annelliertes Pyranon. Die Reaktion zum 3-Bromo-chromanon 15 erfolgte durch direkte Umsetzung mit $\mathrm{Br}_{2}$ in $\mathrm{CCl}_{4}[127]$. Der Nachteil bei derartigen Bromierungen, die Zweitsubstitution, ist hier 
Abbildung 2.10: Syntheseroute nach Garner<smiles>O=C1CCOc2ccccc21</smiles>

14<smiles>CCOC(=S)SC1COc2ccccc2C1=O</smiles>

16

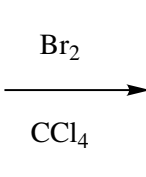

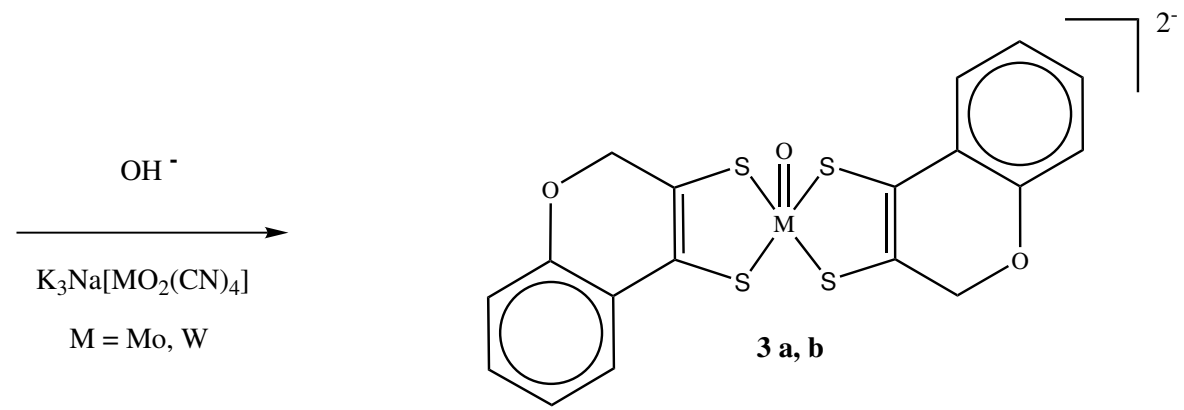<smiles>CCOC(=O)COc1ccccc1C</smiles>

15<smiles>O=c1sc2c(s1)-c1ccccc1OC2</smiles>

17

durch die Struktur des Ausgangsprodukts, in dem die zweite Position durch den aromatischen Ring blockiert ist, ausgeschlossen. Der Vorteil gegenüber herkömmlichen Bromierungsmitteln wie PHT oder NBS [128] ist die wesentlich schnellere Reaktionszeit. Das Produkt konnte in guter Ausbeute erhalten werden. Das NMR-Spektrum zeigt die zu erwartenden Signale der Protonen am Pyranring als Multiplett bei 4.55 bis $4.72 \mathrm{ppm}$ und der aromatischen Protonen im Bereich von 7.00 bis $7.94 \mathrm{ppm}$. Die Elementaranalyse ist in sehr guter Übereinstimmung mit den berechneten Werten.

Die Umsetzung des 3-Bromo-Chromanons zum Xanthogenat 16 erfolgt via $\mathrm{S}_{N} 2$ Reaktion. Die hierfür erforderlichen Bedingungen schränken die Auswahl an Ligandensystemen bezüglich der $\beta$-Keto-Position stark ein. Benachbarte Alkylsubstituen- 
ten, vor allem stark verzweigte, behindern die $\mathrm{S}_{N}$ 2-Reaktivität merklich. Die bereits durch das cyclische Alkylierungsmittel langsam verlaufende Reaktion 11, würde hierbei nochmal beeinträchtigt. So scheiden für diesen Reaktionsweg Ligandvorstufen, die an dieser Position über stark verzweigte Substituenten verfügen, wie z.B. das synthetisch leicht zugängliche Dimethylchromanon, aus. Die versuchten Umsetzungen mit dieser Verbindung führten aufgrund $\mathrm{zu}$ geringer Aubeuten nicht zum gewünschten Dithiocarbonat. In Bezug auf die Modellierung des Kofaktors Molybdopterin liegt in dieser Position die Seitenkette, verbunden mit einem Phosphat. Bei der Auswahl von zukünftigen Liganden, die diese enthalten, könnte das die Reaktion beeinträchtigen. Im Fall des Chromanons verläuft die Reaktion gemäßigt mit mittlerer Ausbeute zum Xanthogenat. Das NMR-Spektrum zeigt die typischen Signale der Ethylgruppe mit einem Triplett bei $1.19 \mathrm{ppm}$ und einem Quartett bei $2.80 \mathrm{ppm}$, das Multiplettmuster der am Pyranring verbliebenen Protonen erscheint etwas weiter aufgespalten als am Bromochromanon als drei Multipletts bei $4.48,4.58$ bis 4.75 und 4.90 bis 5.08 ppm. Die Signale der aromatischen Protonen liegen auch etwas weiter aufgespalten im Bereich von 6.90 bis 8.07 ppm. Die Reinheit ist wiederum durch die Elementaranalyse mit sehr guter Übereinstimmung bestätigt.

Die säurekatalysierte Umlagerung zum Dithiocarbonat 17 ist, besonders bei empfindlichen Systemen wie z.B. dem Pyranylliganden, der problematischste Schritt. Im Gegensatz zu der von Garner verwendeten Schwefelsäure, welche bei diesem System zur Zersetzung führt, gelingt es hier mit $\mathrm{HBr}$, das Produkt in mäßiger Ausbeute zu erhalten. Die viel stärkere Säure HBr scheint nötig zu sein, um die Dithiocarbonateinheit aufzuspalten, während die Umsetzung mit der schwächeren $\mathrm{H}_{2} \mathrm{SO}_{4}$ eine zusätzliche Erwärmung benötigt und somit die Reaktionsbedingungen schwerer zu regulieren sind. Das nach Versetzen mit Eiswasser ausgefallene Produkt ließ sich nach

\footnotetext{
${ }^{11}$ Aufbau von Ringspannung im Übergangszustand im Fall von Sechsringen
} 
Umkristallisation in Ethanol in guter Reinheit erhalten, wie die EA bestätigt.

Im NMR Spektrum ist jetzt neben den aromatischen Protonen nur noch das Singulett der verbliebenen $\mathrm{CH}_{2}$-Protonen des Pyranrings bei $5.02 \mathrm{ppm}$ zu erkennen. Im IR Spektrum sind deutlich die C-O-Streck- bei $1667 \mathrm{~cm}^{-1}$ und Biegeschwingung bei $746 \mathrm{~cm}^{-1} \mathrm{zu}$ erkennen.

Die Umsetzung dieses Liganden mit den Metallkomplexen $\left[\mathrm{MoO}_{2}(\mathrm{CN})_{4}\right]^{4-}$ und $\left[\mathrm{WO}_{2}(\mathrm{CN})_{4}\right]^{4-}$ erfolgt im stark basischen Milieu. Das Dithiocarbonat wird dazu separat mit $\mathrm{KOH}$ zum freien Dithiolen gespalten und zu einer Lösung aus $\left[\mathrm{MoO}_{2}(\mathrm{CN})_{4}\right]^{4-}$ bzw. $\left[\mathrm{WO}_{2}(\mathrm{CN})_{4}\right]^{4-}$ in basisch-wässriger Lösung mit vier Äquivalenten $\mathrm{KOH}$ gegeben. Nach Erhitzen und Zugabe des Gegenions fällt das Produkt in Form eines rotbraunen (Mo) bzw. braunen (W) Feststoffs aus.

Für den Mo-Komplex konnten Einkristalle erhalten werden, die jedoch aufgrund des Vorliegens einer fehlgeordneten Struktur nicht in nötiger Güte gelöst werden konnte. Zwar ist das Strukturelement des gebundenen Dithiolenliganden erkennbar, jedoch lässt die unsichere Datenlage eine weitere Interpretation der Strukturdaten nicht zu. Die Elementaranalyse liegt etwas unter den berechneten Werten und deutet auf eventuell eingelagerte Lösungsmittelmoleküle hin. Aufgrund ihrer schlechten Löslichkeit zeigten weder ESI noch NMR interpretierbare Ergebnisse für diese Verbindungen.

Im IR-Spektrum erscheint die M-O-Streckschwingung bei $1107 \mathrm{~cm}^{-1}$, weiterhin sind die typischen Banden des Aromaten und der C-Atome zu erkennen. Die beim Wolframkomplex analoge W-O Streckschwingung folgt in sehr dichtem Abstand bei $1108 \mathrm{~cm}^{-1}$. Diese Werte sind nahezu identisch mit den M-O-Streckschwingungen der Verbindungen $\left[\mathrm{MoO}(\mathrm{fdt})_{2}\right]^{2-}$ und $\left[\mathrm{WO}(\mathrm{fdt})_{2}\right]^{2-}$ mit je $1107 \mathrm{~cm}^{-1}[112]$. Beim Vergleich mit den aus den DFT-Rechnungen erhaltenen M-O-Streckschwingungen zeigen sich wieder Unterschiede, die auf der Vernachlässigung der anharmonischen Beiträge zurückzuführen sind. Die experimentell bestimmten Schwingungen liegen um etwa 50 
Abbildung 2.11: Kristallstruktur der Verbindung 3a

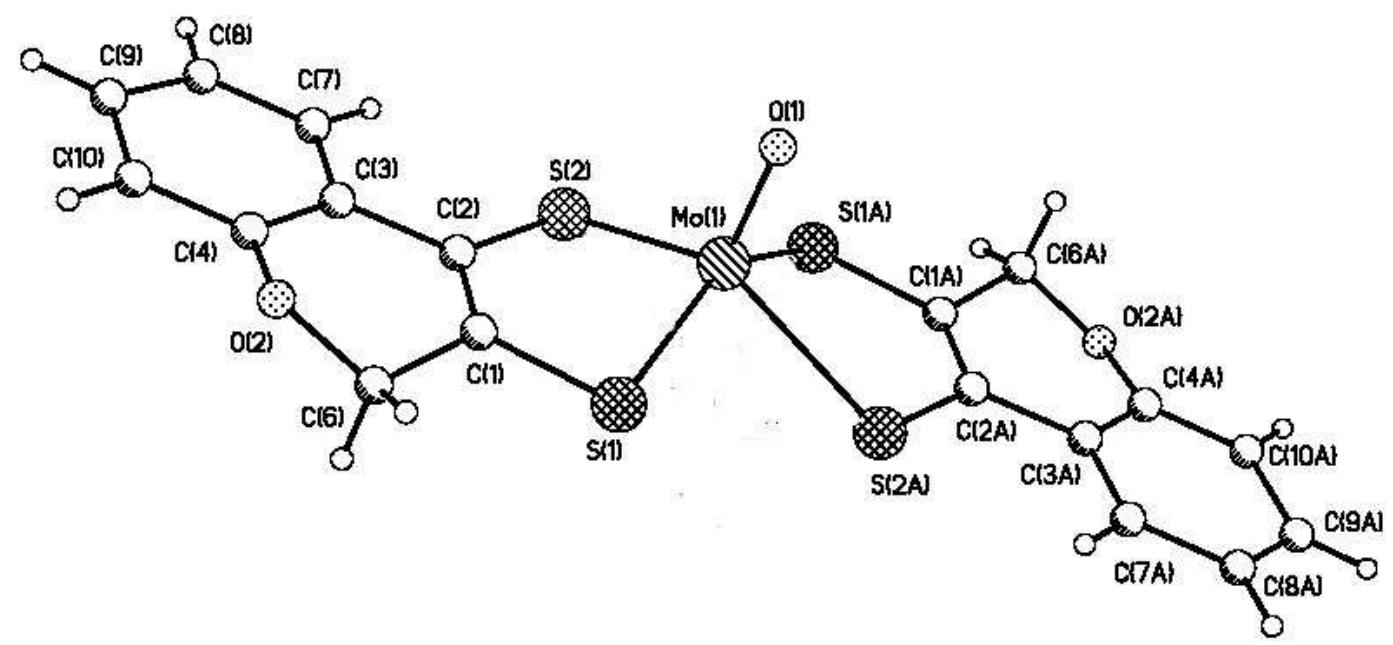

Tabelle 2.10: M-O-Streckschwingungen von $\left[\mathrm{MO}(\mathrm{cdt})_{2}\right]^{2-}$ und $\left[\mathrm{MO}(\mathrm{fdt})_{2}\right]^{2-}(\mathrm{M}=\mathrm{Mo}$, W)

\begin{tabular}{lccl}
\hline & $\nu_{\exp }\left[\mathrm{cm}^{-1}\right]$ & $\nu_{\text {theo }}\left[\mathrm{cm}^{-1}\right]$ & $f^{a}$ \\
\hline$\left[\mathrm{MoO}(\mathrm{cdt})_{2}\right]^{2-}$ & 1108 & 954 & 0.86 \\
{$\left[\mathrm{WO}(\mathrm{cdt})_{2}\right]^{2-}$} & 1107 & 951 & 0.86 \\
{$\left[\mathrm{MoO}(\mathrm{fdt})_{2}\right]^{2-}$} & 1107 & 954 & 0.86 \\
{$\left[\mathrm{WO}(\mathrm{fdt})_{2}\right]^{2-}$} & 1107 & 952 & 0.86
\end{tabular}

${ }^{a}$ Skalierungsfaktor $\frac{\nu_{\text {thee }}}{\nu_{\text {exp }}}$

$\mathrm{cm}^{-1}$ über den berechneten. Der Skalierungsfaktor, der bei allen Komplexen einen gleichen Wert ergibt, untermauert die Zuordung und die Tendenz der Schwingungen, die bei den Wolframkomplexen marginal niedriger ist. Tabelle 2.10 zeigt die M-OStreckschwingungen der Chromanyl- und Flavanylkomplexe im Überblick.

\subsubsection{Geometrische Strukturen}

In Abbildung 2.18 sind exemplarisch die Strukturen der Verbindungen in OS IV, 3a und 4a 12 als Vertreter der Chromanyl- und Flavanylverbindungen gezeigt.

\footnotetext{
${ }^{12}$ Hierbei bezeichnen 3a-c die Verbindungen $\left[\mathrm{MoO}(\mathrm{cdt})_{2}\right]^{z-}$ mit $\mathrm{z}=-2,-1,0$; 4a-c die Verbindungen $\left[\mathrm{WO}(\mathrm{cdt})_{2}\right]^{z-}$ mit $\mathrm{z}=-2,-1,0$; analog dazu 5a-c $\left[\mathrm{MoO}(\mathrm{fdt})_{2}\right]^{z-}$ mit $\mathrm{z}=-2,-1,0$ und 6a-c $\left[\mathrm{WO}(\mathrm{cdt})_{2}\right]^{z-}$ mit $\mathrm{z}=-2,-1,0$.
} 
Abbildung 2.12: Strukturen der Verbindungen $\left[\mathrm{MO}(\mathrm{cdt})_{2}\right]^{2-}$ und $\left[\mathrm{MO}(\mathrm{fdt})_{2}\right]^{2-}$
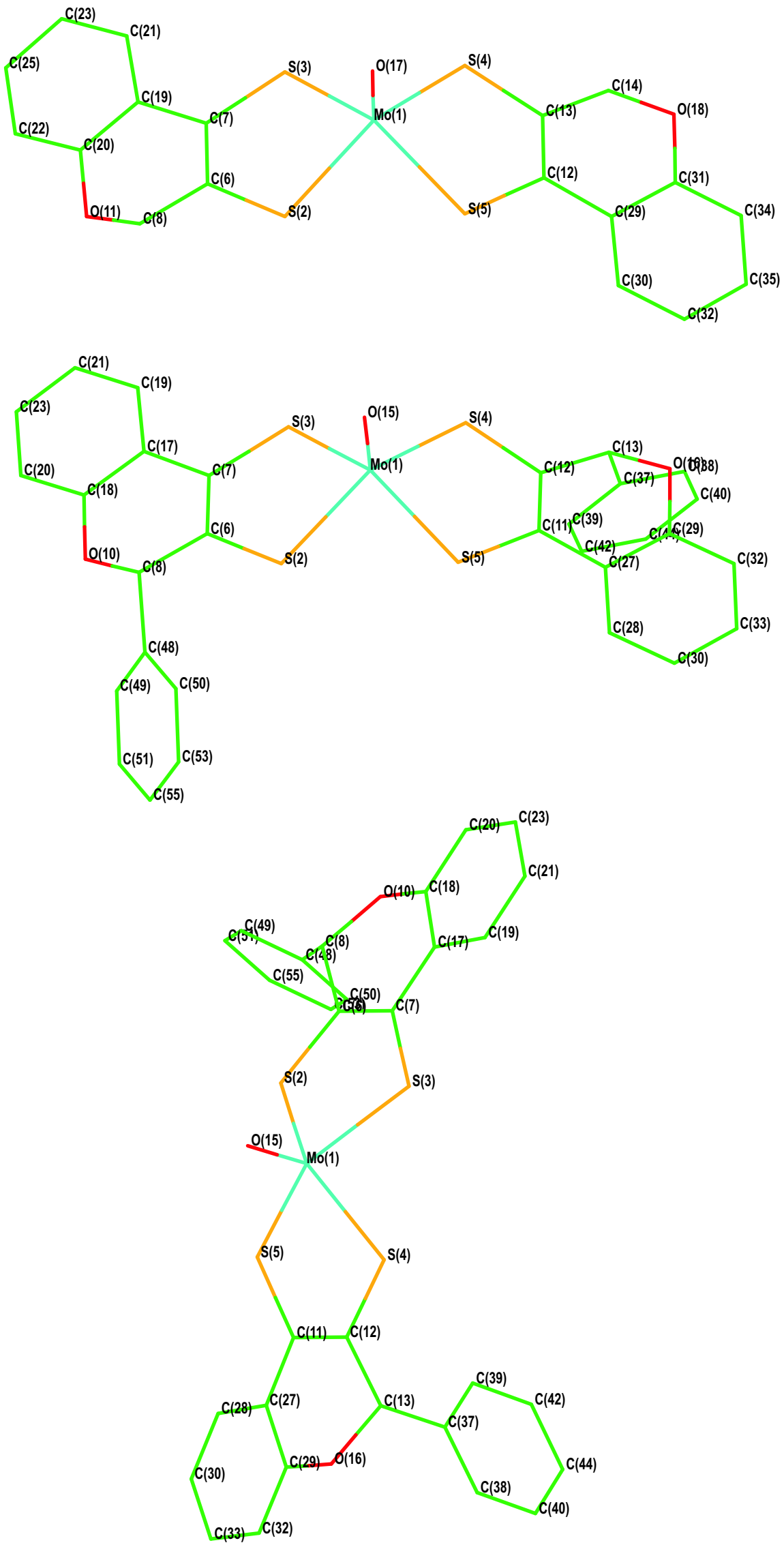
Tabelle 2.11zeigt die wichtigsten das Metallzentrum betreffenden Geometrieparameter der optimierten Strukturen der Chromanyl-Komplexe.

Im Unterschied zu dem im vorangegangenen Kapitel besprochenen Modell verfügt dieser Ligand zusätzlich zum Tetrahydropyranyldithiolen über ein anneliertes Benzol, welches das Pteridingerüst modelliert.

Die für die quadratisch-pyramidal vorliegenden Komplexe typische Anhebung des Metalls über die $\mathrm{S}_{4}$-Ebene ist bei diesem Liganden etwas stärker als bei den Pyranylkomplexen. Die Werte steigen mit zunehmender OS von $0.791 \AA(\mathrm{Mo}) / 0.731 \AA(\mathrm{W})$ auf $0.845 \AA(\mathrm{Mo}) / 0.809 \AA(\mathrm{W})$ an und fallen somit beim Wolfram wiederum geringer aus, obwohl der Anstieg insgesamt beim Wolfram höher als beim Molybdän ist.

Der beobachtete Faltungswinkel beinhaltet wieder einen deutlichen Anstieg beim Wechsel von OS IV zu V von $12.91^{\circ}$ auf $31.22^{\circ}$ (Mo) bzw. $11.99^{\circ}$ auf $30.87^{\circ}$ (W) und einen nochmaligen etwas geringeren Anstieg auf $36.34^{\circ}$ (Mo) und $42.03^{\circ}$ (W) beim Wechsel zur OS VI. Der nicht von der Faltung betroffene Ligand, dessen Abknickung bei maximal $15^{\circ}$ (4c) liegt durchläuft dabei ebenso eine von der OS abhängige Schwankung. So liegen die Werte für den nahezu symmetrischen Zustand in OS IV bei $13.54^{\circ} / 12.01^{\circ}(\mathrm{Mo} / \mathrm{W})$ und verringern sich in der OS auf $9.15^{\circ}$ (Mo) bzw. $2.32^{\circ}$ (W) während sie in OS VI wieder nah bei ihren ursprünglichen Werten von $11.01^{\circ} \mathrm{bzw}$ $15.00^{\circ}$ liegen. In Bezug auf den Faltungsvorgang beobachtet man wiederum beim axialen Sauerstoff eine Neigung zu Gunsten des nicht abknickenden Liganden. Der Winkel bezüglich des S-S-Vektors bleibt mit Werten um die $114^{\circ}$ beim Wechsel von OS IV zu OS V nahezu konstant (minimale Verringerung auf $113.09^{\circ} / 111.37^{\circ}(\mathrm{Mo} / \mathrm{W})$ beim Wechsel in OS VI), während sich der Winkel in Richtung des gefalteten Liganden auf $122.98^{\circ} / 120.96^{\circ}(\mathrm{Mo} / \mathrm{W})$ in OS VI vergrößert.

Auch hier erfolgt eine Verkürzung der Bindungslängen M(1)-O(17), M(1)-S(2-5) und S-C mit zunehmender Oxidationsstufe. Der Abstand $\mathrm{M}(1)-\mathrm{O}(17)$ verringert sich 
Tabelle 2.11: Ausgewählte Geometrieparameter $\left[\mathrm{MO}(\mathrm{cdt})_{2}\right]^{z-}(\mathrm{M}=\mathrm{Mo}, \mathrm{W}, \mathrm{z}=-2,-1$, $0)$

\begin{tabular}{lcccccc}
\hline & $\mathbf{3 a}$ & $\mathbf{3 b}$ & $\mathbf{3 c}$ & $\mathbf{4 a}$ & $\mathbf{4 b}$ & $\mathbf{4 c}$ \\
\hline $\mathrm{M}(1)-\mathrm{S}$ \\
$\mathrm{M}(1)-\mathrm{O}(17)$ & $0.791(6)$ & $0.810(8)$ & $0.845(4)$ & $0.730(7) 3$ & $0.774(8)$ & $0.809(1)$ \\
$\mathrm{M}(1)-\mathrm{S}(2)$ & 1.7093 & 1.7047 & 1.6971 & 1.7206 & 1.7127 & 1.7055 \\
$\mathrm{M}(1)-\mathrm{S}(3)$ & 2.4726 & 2.4529 & 2.4304 & 2.45143 & 2.4415 & 2.4161 \\
$\mathrm{M}(1)-\mathrm{S}(4)$ & 2.4611 & 2.4406 & 2.4303 & 2.4428 & 2.4317 & 2.4178 \\
$\mathrm{M}(1)-\mathrm{S}(5)$ & 2.4725 & 2.4392 & 2.4174 & 2.4524 & 2.4340 & 2.4028 \\
$\mathrm{~S}(2)-\mathrm{C}(6)$ & 2.4566 & 2.4295 & 2.4084 & 2.4386 & 2.4233 & 2.3954 \\
$\mathrm{~S}(3)-\mathrm{C}(7)$ & 1.7590 & 1.7449 & 1.7145 & 1.7662 & 1.7550 & 1.7289 \\
$\mathrm{~S}(4)-\mathrm{C}(13)$ & 1.7877 & 1.7642 & 1.7360 & 1.7949 & 1.77434 & 1.7483 \\
$\mathrm{~S}(5)-\mathrm{C}(12)$ & 1.7590 & 1.7553 & 1.7268 & 1.7663 & 1.7615 & 1.7386 \\
$\mathrm{C}(6)-\mathrm{C}(7)$ & 1.7877 & 1.7749 & 1.7488 & 1.79512 & 1.7806 & 1.7583 \\
$\mathrm{C}(12)-\mathrm{C}(13)$ & 1.3546 & 1.3574 & 1.3811 & 1.3519 & 1.3513 & 1.3693 \\
$\mathrm{O}(17)-\mathrm{M}(1)-[\mathrm{S}(2)-\mathrm{S}(3)]$ & 114.48 & 117.74 & 122.98 & 113.67 & 116.42 & 120.96 \\
$\mathrm{O}(17)-\mathrm{M}(1)-[\mathrm{S}(4) \mathrm{S}(5)]$ & 114.07 & 114.36 & 113.09 & 113.27 & 113.28 & 111.37 \\
$\mathrm{FW} \mathrm{M}(1)-\mathrm{S}(2)-\mathrm{S}(3)$ & 13.54 & 9.15 & 11.01 & 12.01 & 2.32 & 15.00 \\
$\mathrm{FW} \mathrm{M}(1)-\mathrm{S}(4)-\mathrm{S}(5)$ & 12.91 & 31.22 & 36.34 & 11.99 & 30.87 & 42.03 \\
$\mathrm{C}(8)-\mathrm{O}(11)$ & 1.4453 & 1.4369 & 1.4301 & 1.4465 & 1.4363 & 1.4299 \\
$\mathrm{O}(11)-\mathrm{C}(20)$ & 1.3651 & 1.3659 & 1.3624 & 1.3651 & 1.3660 & 1.3645 \\
$\mathrm{C}(14)-\mathrm{O}(18)$ & 1.4440 & 1.4383 & 1.4316 & 1.4452 & 1.4356 & 1.4315 \\
$\mathrm{O}(18)-\mathrm{C}(31)$ & 1.3653 & 1.3654 & 1.3609 & 1.3653 & 1.3652 & 1.3628 \\
$\mathrm{C}(7)-\mathrm{C}(6)-\mathrm{C}(8)-\mathrm{O}(11)$ & 28.28 & -31.47 & 34.73 & 28.02 & 30.81 & 34.45 \\
$\mathrm{C}(12)-\mathrm{C}(13)-\mathrm{C}(14)-\mathrm{O}(18)$ & -28.41 & 30.86 & 32.56 & -27.83 & -29.96 & 32.95 \\
& & & & & &
\end{tabular}

bei den Molybdänverbindungen von $1.7093 \AA$ auf $1.6971 \AA$ um $0.012 \AA$ und ist bei den Wolframverbindungen mit einer Änderung um $0.015 \AA$ von $1.7206 \AA$ auf 1.7055 A marginal größer.

Die Metall-Schwefel-Bindungen liegen im Bereich von 2.395 (4c) bis 2.473 (3a) und nehmen mit steigender Oxidation in unterschiedlicher Weise ab. Wiederum sind die W-S-Bindungen in allen OS um durchschnittlich $0.02 \AA$ kürzer als die Mo-SBindungen. Das unterschiedliche Verhalten der vier Metall-Schwefel-Bindungen ist in allen Oxidationsstufen zu beobachten. In OS IV sind die zu einem Liganden gehörigen S-Atome unterschiedlich lang während sich die gegenüberstehenden S-Atome in ihrer Länge ähneln. So betragen die Bindungslängen von M-S(2) und M-S(4) $2.473 \AA$ (Mo) 
und $2.452 \AA(\mathrm{W})$ und die jeweils benachbarten Bindungslängen M-S(3) und M-S(5) $2.461 / 2.457 \AA$ (Mo) und 2.443/2.439 $\AA$ (W). In OS V erfolgt eine Verkürzung aller Metall-Schwefel-Bindungen, wobei sich die Bindungslängen der direkt gegenüberliegenden S-Atome bei M-S(3) und M-S(4) stärker ähneln. In OS VI wiederum sind die jeweils zu einem Liganden gehörigen S-Atome mit ähnlichen Abständen an das Metall gebunden, so mit $2.430 \AA$ bei Mo-S(2) und Mo-S(3) und $2.417 / 2.408 \AA$ bei Mo-S(4) und Mo-S(5). Dieses Verhalten ist bei den Wolframverbindungen analog, nur sind die Bindungen insgesamt kürzer.

Die stärkste Verkürzung erfolgt wiederum bei den S-C-Bindungen von ca. $1.79 \AA$ (3a) auf $1.73 \AA(\mathbf{3 c})$. Dabei fällt ein deutlicher Unterschied zwischen den zu einem Liganden gehörenden S-C-Bindungen auf, der in allen Oxidationsstufen beobachtet wird. Es sind jeweils die zueinander trans-ständigen Bindungen $\mathrm{S}(2)-\mathrm{C}(6)$ und $\mathrm{S}(4)$ $\mathrm{C}(13)$ mit $1.759 / 1.766 \AA(\mathbf{3 a} / \mathbf{4 a})$ sowie $\mathrm{S}(3)-\mathrm{C}(7)$ und $\mathrm{S}(5)-\mathrm{C}(12)$ mit $1.788 / 1.795 \AA$ (3a/4a) gleich lang. Das Paar $\mathrm{S}(2)-\mathrm{C}(6) / \mathrm{S}(4)-\mathrm{C}(13)$ ist in jeder OS und bei beiden Metallen um etwa 0.02 bis $0.03 \AA$ kürzer.

Die C-C-Doppelbindung liegt in Bereichen von 1.355/1.352 $\AA$ (OS IV, Mo/W) bis 1.387/1.380 $\AA$ (OS VI, Mo/W) und ist damit länger als eine C-C-Doppelbindung im Ethen $(1.34 \AA$ ). Dabei sind die Werte für beide Liganden in den OS IV und V jeweils gleich lang, in OS VI ist die Bindung $\mathrm{C}(6)-\mathrm{C}(7)$ bei der Molybdänverbindung um $0.005 \AA$ kürzer und bei der Wolframverbindung um $0.01 \AA$ als die gegenüberliegende Bindung C(12)-C(13), wobei sich die längeren Bindungen am gefalteten Liganden befinden.

Bei der Betrachtung der C-O-Bindungen innerhalb des Pyranrings zeigen sich deutliche Unterschiede zu den nur das Pyran enthaltenen Komplexen. Während die näher an der Dithioleneinheit liegende C-O-Bindung $\mathrm{C}(8)-\mathrm{O}(9)$ und $\mathrm{C}(12)-\mathrm{O}(14)$ mit Werten von durchschnittlich $1.45 \AA$ (Mo u. W) nur etwas länger ist als die vergleichbare 
Bindung in den Pyranylkomplexen, sind die direkt neben dem annellierten Benzolring liegenden C-O-Bindungen $\mathrm{O}(11)-\mathrm{C}(20)$ und $\mathrm{O}(18)-\mathrm{C}(31)$ mit Werten von durchschnittlich 1.36 A stark verkürzt. Im Gegensatz zu den Pyranylkomplexen, deren C-OBindungen mit zunehmender Oxidation einmal zu- (C-O neben der Dithioleneinheit) und einmal abnahmen (C-O im Ring) zeigt sich hier bei beiden eine Abnahme der Bindungslänge. In diesen Systemen macht sich der Einfluss des aromatischen Rings bemerkbar. So beträgt der Wert für die Bindung $\mathrm{C}(8)-\mathrm{O}(11)$, die sich näher an der Dithioleneinheit befindet, $1.4453 \AA$ (OS IV, Mo), sinkt bei der Oxidation auf 1.4301 $\AA$ (OS VI, Mo) ab und liegt damit in einem ähnlichen Bereich wie im Pyranylkomplex. Die bereits an den Aromaten grenzende Bindung $\mathrm{C}(20)-\mathrm{O}(11)$ dagegen beträgt nur noch $1.3651 \AA(\mathrm{OS} \mathrm{IV}, \mathrm{Mo})$ und verkürzt sich auf $1.3634 \AA$ (OS VI, Mo). D.h. dass diese Bindung zwar im Vergleich zu den Pyranylkomplexen deutlich kürzer ist, jedoch im Oxidationsprozess nahezu konstant bleibt. Die entsprechenden Werte für die Wolframverbindungen verhalten sich analog.

Die Halbsessel-Konformation des Pyranrings ist im Gegensatz zu den Pyranylkomplexen hier deutlich stärker ausgeprägt. Das Hervorstehen des Sauerstoff-Atoms aus dem Ring in endo- bzw. exo-Form scheint auch hier keinem Trend zu unterliegen. So liegen die Sauerstoff-Atome des linken Liganden bis auf $\mathbf{3 b}$ komplett in endo-Form vor. In den OS IV und V ist wiederum das wechselseitige Verhalten zu beobachten, dass, wenn in einem Liganden die endo-Form vorlieg, in dem anderen Liganden die exo-Form eingenommen wird und umgekehrt. In OS VI dagegen sind in beiden Liganden des jeweiligen Mo- und W-Komplexes die Sauerstoff-Atome exo-ständig. Die stärker ausgeprägte Konformation wird an den hohen Werten des Diederwinkels C(7)$\mathrm{C}(6)-\mathrm{C}(8)-\mathrm{O}(11)$, der zwischen $28.23^{\circ}$ bzw. $28.02^{\circ}$ (OS IV, Mo bzw. W) und $34.73^{\circ}$ bzw. 34.45 (OS VI Mo bzw. W) liegt, deutlich. Wiederum ist ein gleichmäßiges An- 
Tabelle 2.12: Ausgewählte Geometrieparameter des arom. Ringes

\begin{tabular}{lcccccc}
\hline & $\mathbf{3 a}$ & $\mathbf{3 b}$ & $\mathbf{3 c}$ & $\mathbf{4 a}$ & $\mathbf{4 b}$ & $\mathbf{4 c}$ \\
\hline $\mathrm{C}(20)-\mathrm{C}(22)^{a}$ & 1.3915 & 1.3917 & 1.3932 & 1.3915 & 1.3916 & 1.3923 \\
$\mathrm{C}(19)-\mathrm{C}(21)$ & 1.4046 & 1.4012 & 1.4052 & 1.4044 & 1.4012 & 1.4036 \\
$\mathrm{C}(23)-\mathrm{C}(25)$ & 1.3929 & 1.3935 & 1.3984 & 1.3929 & 1.3933 & 1.3970 \\
$\mathrm{C}(20)-\mathrm{C}(22)-\mathrm{C}(25)$ & 120.33 & 121.11 & 120.92 & 121.38 & 120.01 & 120.92 \\
$\mathrm{C}(19)-\mathrm{C}(21)-\mathrm{C}(23)$ & 121.84 & 117.69 & 118.10 & 116.95 & 121.41 & 121.0 \\
$\mathrm{C}(20)-\mathrm{C}(22)-\mathrm{C}(25)-\mathrm{C}(23)$ & -0.37 & 0.21 & 0.00 & -0.37 & -0.16 & 0.02 \\
${ }^{a}$ nur der Ring eines Liganden ist dargestellt, der des anderen verhält sich analog
\end{tabular}

steigen der Werte mit zunehmender OS zu beobachten 13 .

Wichtige Geometrieparameter des an den Pyranring geknüpften Benzolringes sind in Tabelle 2.12 zusammengestellt.

Die Werte der C-C-Bindungslängen von 1.39 bis $1.40 \AA$, die sie einschließenden Winkel von ca. $120^{\circ}$ und die durch den zu Null tendierenden Diederwinkel beschriebene Planarität des Ringes ist in sehr guter Übereinstimmung mit den Werten der Bindungslängen und -Winkel des freien Benzols mit einer C-C Bindungslänge von $1.39 \AA$ und einem C-C-C-Winkel von $120^{\circ}$ [118]. Auswirkungen des Redoxprozesses werden anhand der Geometrie nicht beobachtet, die Werte bleiben in allen drei OS konstant, abgesehen von einer geringfügigen Schwankung des Winkels C(19)-C(21)$\mathrm{C}(23)$ zwischen $121^{\circ}$ und $117^{\circ}$.

Die strukturellen Parameter der Flavanylkomplexe sind bezüglich der beiden Systemen gemeinsamen Komponenten in Übereinstimmung mit denen der Chromanylkomplexe. Die relevanten Bindungslängen sind nahezu identisch und weichen erst im Bereich der dritten Nachkommastellen geringfügig ab. So sind auch die beobachteten Trends der das Metallzentrum betreffenden Bindungen analog. Geringere Abweichungen von 1-2 ${ }^{\circ}$ sind lediglich bei den Winkeln des an den Pyranring gebundenen Benzols sichtbar, welche sich bei $\mathrm{C}(19)-\mathrm{C}(21)-\mathrm{C}(23)$ knapp unterhalb des Literaturwerts für das Benzol befinden. Bei den Chromanylkomplexen liegt dieser Winkel ohne erkennba-

\footnotetext{
${ }^{13}$ Betrachtet werden die Beträge der Winkel.
} 
ren Trend bei den Verbindungen 2a und 3a bei $118^{\circ}$ und bei $\mathbf{4 a}$ bei $117^{\circ}$, die restlichen Verbindungen 1a, 5a und $\mathbf{6 a}$ liegen mit $121^{\circ}$ etwas über dem Wert vom Benzol. Bei den Flavanylverbindungen dagegen liegen die Winkel für alle Verbindungen unter $120^{\circ}$ und steigen von $117^{\circ}$ auf $118^{\circ}$ mit zunehmender OS an.

Größere Unterschiede zeigen sich in der Konformation des Pyranringes, wobei die Richtung der Konformation identisch ist. In OS IV ist der Diederwinkel C(7)-C(6)$\mathrm{C}(8)-\mathrm{O}(10)$ bei den Flavanyl-Verbindungen mit $22.30^{\circ}$ (OS IV, Mo) um 7-10 geringer als der des vergleichbaren Chromanylkomplexes, während die Abweichungen in OS V und VI mit $33.42^{\circ}$ und $36.30^{\circ}$ kleiner sind (ca. $2^{\circ}$ ). Dies ist dem unmittelbar an $\mathrm{C}(8)$ des Pyranringes geknüften Phenylrest geschuldet, der einen merklichen Einfluss auf die Konformation desselben aufweist.

Der Phenylrest liegt willkürlich innerhalb der Startgeometrie gewählten Richtung unterhalb des Pyranringes und nimmt eine der Konformation des Sauerstoff-Atoms entgegengesetzte Position ein, um die Abstoßungen zu minimieren.

Die Länge der Bindung C(8)-C(48) mit der der Phenylrest an den Pyranring gebunden ist reicht von $1.521 \AA$ in OS IV (Mo) bis zu $1.513 \AA$ in OS VI (Mo) und liegt damit unter dem Wert einer C-C-Einfachbindung von $1.54 \AA$, was auf den aromatischen Einfluss des Phenylrings zurückzuführen ist liegt. Die Verkürzung mit zunehmender Oxidation ist wieder bei beiden Liganden identisch. Der Winkel zum Pyranring, $\mathrm{O}(10)-\mathrm{C}(8)-\mathrm{C}(48)$ liegt mit Werten von $104.05^{\circ}$ (OS IV), $109.40^{\circ}$ (OS V) und $107.22^{\circ}$ (OS VI, Mo) nah am Tetraederwinkel während der Winkel C(6)-C(8)-C(48) mit nahezu konstant bleibenden $115^{\circ}$ deutlich darüber liegt.

Um die Ausrichtung des planar vorliegenden Phenylrings zu beschreiben wurden die Diederwinkel C(6)-C(8)-C(48)-C(49) und S(2)-S(3)-C(8)-C(48) gewählt. Ersterer beschreibt die Ausrichtung gegenüber der planar angenommenen C-C-DoppelbindungsEbene und letzterer die Ausrichtung gegenüber der Dithiolen-Ebene. Dabei schwanken 
Tabelle 2.13: Geometrieparameter der aromatischen Ringe von $\left[\mathrm{MO}(\mathrm{fdt})_{2}\right]^{z-}$

$(\mathrm{M}=\mathrm{Mo}, \mathrm{W}, \mathrm{z}=-2,-1,0]$

\begin{tabular}{lcccccc}
\hline & $\mathbf{5 a}$ & $\mathbf{5 b}$ & $\mathbf{5 c}$ & $\mathbf{6 a}$ & $\mathbf{6 b}$ & $\mathbf{6 c}$ \\
\hline $\mathrm{C}(7)-\mathrm{C}(6)-\mathrm{C}(8)-\mathrm{O}(10)$ & 21.30 & -33.42 & 36.30 & 19.57 & 29.20 & 35.57 \\
$\mathrm{C}(11)-\mathrm{C}(12)-\mathrm{C}(13)-\mathrm{O}(16)$ & -33.69 & 30.51 & 33.36 & -33.38 & -30.40 & 32.96 \\
$\mathrm{C}(18)-\mathrm{C}(20)$ & 1.3921 & 1.3916 & 1.3933 & 1.3923 & 1.3917 & 1.3925 \\
$\mathrm{C}(17)-\mathrm{C}(19)$ & 1.4045 & 1.4013 & 1.4052 & 1.4042 & 1.4017 & 1.4037 \\
$\mathrm{C}(20)-\mathrm{C}(18)-\mathrm{C}(17)$ & 121.58 & 121.11 & 121.01 & 121.51 & 121.33 & 121.03 \\
$\mathrm{C}(18)-\mathrm{C}(17)-\mathrm{C}(19)$ & 116.99 & 117.81 & 118.15 & 117.05 & 117.66 & 118.19 \\
$\mathrm{C}(18)-\mathrm{C}(20)-\mathrm{C}(23)-\mathrm{C}(21)$ & -0.33 & 0.30 & 0.06 & -0.33 & -0.12 & 0.08 \\
$\mathrm{C}(29)-\mathrm{C}(32)$ & 1.3911 & 1.3916 & 1.3928 & 1.3911 & 1.3919 & 1.3921 \\
$\mathrm{C}(27)-\mathrm{C}(28)$ & 1.4044 & 1.4017 & 1.4055 & 1.4042 & 1.4018 & 1.4041 \\
$\mathrm{C}(39)-\mathrm{C}(29)-\mathrm{C}(27)$ & 121.51 & 121.29 & 120.98 & 121.47 & 121.14 & 120.97 \\
$\mathrm{C}(29)-\mathrm{C}(27)-\mathrm{C}(28)$ & 117.03 & 117.72 & 118.18 & 117.09 & 117.76 & 118.28 \\
$\mathrm{C}(29)-\mathrm{C}(32)-\mathrm{C}(33)-\mathrm{C}(30)$ & 0.28 & -0.23 & -0.12 & 0.30 & 0.13 & -0.14 \\
$\mathrm{C}(8)-\mathrm{C}(48)$ & 1.5206 & 1.5319 & 1.5117 & 1.5211 & 1.5161 & 1.5121 \\
$\mathrm{O}(10)-\mathrm{C}(8)-\mathrm{C}(48)$ & 104.05 & 109.40 & 107.22 & 104.03 & 105.47 & 107.10 \\
$\mathrm{C}(6)-\mathrm{C}(8)-\mathrm{C}(48)$ & 115.24 & 115.11 & 115.58 & 115.21 & 115.34 & 115.41 \\
$\mathrm{C}(6)-\mathrm{C}(8)-\mathrm{C}(48)-\mathrm{C}(49)$ & 129.29 & 179.31 & 106.76 & 130.98 & 116.91 & 108.31 \\
$\mathrm{~S}(2)-\mathrm{S}(3)-\mathrm{C}(8)-\mathrm{C}(48)$ & 144.24 & 105.66 & 160.36 & 142.92 & 151.53 & 158.88 \\
$\mathrm{C}(13)-\mathrm{C}(37)$ & 1.5366 & 1.5159 & 1.5129 & 1.5364 & 1.5301 & 1.5132 \\
$\mathrm{O}(16)-\mathrm{C}(13)-\mathrm{C}(37)$ & 108.64 & 105.88 & 106.93 & 108.64 & 109.19 & 106.87 \\
$\mathrm{C}(12)-\mathrm{C}(13)-\mathrm{C}(37)$ & 115.04 & 115.15 & 115.22 & 115.01 & 114.85 & 114.92 \\
$\mathrm{C}(11)-\mathrm{C}(13)-\mathrm{C}(37)-\mathrm{C}(38)$ & -175.43 & 109.30 & 107.19 & -175.02 & 166.72 & 107.09 \\
$\mathrm{~S}(4)-\mathrm{S}(5)-\mathrm{C}(13)-\mathrm{C}(37)$ & 104.53 & 152.99 & 156.72 & 104.74 & -145.52 & 155.71
\end{tabular}

die Werte über große Bereiche, so dass Winkel zwischen ca. 100 und $180^{\circ}$ vertreten sind. Die Werte folgen dabei keinem Trend und weisen auch keine Ähnlichkeiten zwischen den Molybdän- und Wolframverbindungen auf. Dass der Phenylring verschiedenste Positionen einnehmen kann, zeigt deutlich die hohe Flexibilität dieser Position. In Hinblick auf die im Enzym an dieser Position vorliegende Seitenkette ist das eine entscheidende Größe, für die vertretene Meinung eines nicht-starren, flexiblen Systems [115]. 
Tabelle 2.14: NPA-Ladungen von $\left[\mathrm{MO}(\mathrm{cdt})_{2}\right]^{z-}$ und $\left[\mathrm{MO}(\mathrm{fdt})_{2}\right]^{z-}$

\begin{tabular}{|c|c|c|c|c|c|c|}
\hline \multicolumn{7}{|c|}{$\left[\mathrm{MO}(\mathrm{cdt})_{2}\right]^{z-}(\mathrm{M}=\mathrm{Mo}, \mathrm{W} ; \mathrm{z}=-2,-1,0)$} \\
\hline & $3 a$ & $3 b$ & $3 \mathrm{c}$ & $4 \mathbf{a}$ & $4 \mathrm{~b}$ & $4 c$ \\
\hline $\mathrm{M}(1)$ & 0.54 & 0.71 & 0.54 & 0.69 & 0.94 & 0.87 \\
\hline $\mathrm{O}(17)$ & -0.57 & -0.53 & -0.46 & -0.67 & -0.62 & -0.57 \\
\hline $\mathrm{S}(2)$ & -0.16 & -0.06 & 0.11 & -0.18 & -0.10 & 0.06 \\
\hline $\mathrm{S}(3)$ & -0.20 & -0.07 & 0.08 & -0.22 & -0.12 & 0.04 \\
\hline $\mathrm{S}(4)$ & -0.17 & -0.07 & 0.10 & -0.18 & -0.10 & 0.05 \\
\hline $\mathrm{S}(5)$ & -0.20 & -0.08 & 0.07 & -0.21 & -0.11 & 0.04 \\
\hline $\mathrm{C}(6)$ & -0.19 & -0.20 & -0.18 & -0.19 & -0.19 & -0.19 \\
\hline $\mathrm{C}(7)$ & -0.23 & -0.20 & -0.17 & -0.22 & -0.20 & -0.17 \\
\hline $\mathrm{C}(12)$ & -0.23 & -0.20 & -0.16 & -0.22 & -0.20 & -0.16 \\
\hline $\mathrm{C}(13)$ & -0.19 & -0.20 & -0.17 & -0.20 & -0.19 & -0.18 \\
\hline \multicolumn{7}{|c|}{$\left[\mathrm{MO}(\mathrm{fdt})_{2}\right]^{z-}(\mathrm{M}=\mathrm{Mo}, \mathrm{W} ; \mathrm{z}=-2,-1,0)$} \\
\hline & $5 \mathbf{a}$ & $5 \mathrm{~b}$ & 5c & $6 \mathbf{a}$ & $6 \mathrm{~b}$ & 6c \\
\hline $\mathrm{M}(1)$ & 0.55 & 0.68 & 0.56 & 0.69 & 0.95 & 0.88 \\
\hline $\mathrm{O}(15)$ & -0.58 & -0.51 & -0.49 & -0.66 & -0.63 & -0.58 \\
\hline $\mathrm{S}(2)$ & -0.14 & -0.04 & 0.14 & -0.16 & -0.08 & 0.09 \\
\hline $\mathrm{S}(3)$ & -0.19 & -0.07 & 0.08 & -0.20 & -0.11 & 0.03 \\
\hline $\mathrm{S}(4)$ & -0.16 & -0.05 & 0.13 & -0.17 & -0.09 & 0.08 \\
\hline $\mathrm{S}(5)$ & -0.19 & -0.08 & 0.07 & -0.20 & -0.11 & 0.04 \\
\hline $\mathrm{C}(6)$ & -0.20 & -0.20 & -0.18 & -0.21 & -0.19 & -0.19 \\
\hline $\mathrm{C}(7)$ & -0.22 & -0.20 & -0.17 & -0.21 & -0.19 & -0.17 \\
\hline $\mathrm{C}(11)$ & -0.22 & -0.20 & -0.17 & -0.21 & -0.19 & -0.16 \\
\hline $\mathrm{C}(12)$ & -0.21 & -0.19 & -0.18 & -0.22 & -0.20 & -0.18 \\
\hline
\end{tabular}

\subsubsection{Elektronische Struktur und Bindung}

Die NPA-Ladungen der wichtigsten, das Metallzentrum betreffenden Atome sind in Tabelle 2.14 dargestellt. Die Werte sind generell etwas niedriger als bei den Pyranylkomplexen. Die zuvor beobachteten Trends sind jedoch auch bei diesen Systemen deutlich erkennbar.

Die positiviert vorliegenden Metall-Atome unterscheiden sich dabei grundlegend in ihren Beiträgen sowie beim Wechsel der OS. So ist das Wolfram-Atom in OS IV um etwa 0.14 Einheiten stärker positiviert als das Molybdän-Atom, in OS V sogar um 
0.24/0.26 und in OS VI um 0.33/0.32 Ladungseinheiten (Chromanyl-/Flavanylverbindung). Beide Metalle erfahren eine Positivierung beim Wechsel von OS IV zu V von 0.54/0.69 auf 0.71/0.94 Ladungseinheiten (Chromanylverbindung, Mo/W) und einen Rückgang der positiven Ladung beim Schritt zur OS VI zu 0.54/0.87 Ladungseinheiten, die Ladung von Wolfram in OS IV liegt dabei anders als beim Molybdän über dem Wert in OS IV. Die das Metall direkt umgebenden Atome werden ebenso positiviert. Die Nachbaratome des Wolframs sind dabei in allen OS negativer geladen als die des Molybdäns. Die Auswirkungen auf die Ladungsänderung sind beim Schwefel am höchsten. So steigt dessen Ladung von ca. -0.2 bis in den positiven Bereich von etwa +0.1 Ladungseinheiten. Die Sauerstoffatome verändern ihren Ladungsanteil noch um ca. 0.1 Einheiten während die Auswirkungen auf die C-C-Doppelbindung mit Abnahmen um etwa 0.05 Einheiten weitaus geringer ausfallen. Selbst im angrenzenden Pyranring sind, wenn auch von sehr geringem Ausmaß, Änderungen erkennbar, z.B. am Pyran-Sauerstoff mit einer Änderung von 0.03 Einheiten. Eine Ausnahme stellen die beiden den Pyranring mit dem Benzolring verknüpfenden C-Atome C(15) (am S(2)S(3)-Ring) und C(22) (am S(4)-S(5)-Ring) dar, deren Ladung von -0.10 auf -0.15 Ladungseinheiten absinkt. Insgesamt verdeutlichen diese Werte die Einbeziehung des gesamten Ligandensystems, vor allem aber der das Metall direkt umgebenden Atome, in den Redoxvorgang.

Die wichtigsten aus der NBO-Analyse resultierenden Parameter sind in den Tabellen 2.15] und 2.16] dargestellt.

Die Komplexe liegen in der typischen Form vor: das Metall ist mit dem SauerstoffAtom über eine formale Dreifachbindung verknüpft, wobei ein freies Elektronenpaar des Sauerstoffs für die Bindung verwendet wird. Das ist bei beiden Systemen (Chromanyl und Flavanyl) beiden Metallen und in jeder Oxidationsstufe der Fall. Das Vorliegen der Dithioleneinheit, bei der der Schwefel einfach über eine $\sigma$-Bindung an das 
Tabelle 2.15: Bindungsparameter der M-S-Doppelbindungen $\left[\mathrm{M}^{V I} \mathrm{O}(\mathrm{cdt})_{2}\right](\mathrm{M}=\mathrm{Mo}$, W)

\begin{tabular}{|c|c|c|c|c|c|c|c|c|c|c|}
\hline \multirow[t]{2}{*}{ A-B } & & \multirow[t]{2}{*}{$\% \mathrm{~A}$} & \multicolumn{3}{|c|}{ Hybridisierung } & \multirow[t]{2}{*}{$\mathrm{B}^{a}$} & \multirow[t]{2}{*}{$\% \mathrm{~B}$} & \multicolumn{3}{|c|}{ Hybridisierung } \\
\hline & & & $\mathrm{s}$ & $\mathrm{p}$ & $\mathrm{d}$ & & & $\mathrm{s}$ & $\mathrm{p}$ & $\mathrm{d}$ \\
\hline $\mathrm{Mo}(1)-\mathrm{S}(2)$ & $\sigma$ & 23.77 & 11.49 & 0.72 & 87.78 & & 73.10 & 16.44 & 83.42 & 0.15 \\
\hline \multirow[t]{3}{*}{$\mathrm{Mo}(1)-\mathrm{S}(2)$} & $\pi$ & 46.30 & 0.05 & 0.08 & 99.87 & & 47.71 & 0.11 & 99.77 & 0.12 \\
\hline & & & & & & $\mathrm{C}(6)$ & $<2$ & & & \\
\hline & & & & & & $\mathrm{C}(7)$ & $<2$ & & & \\
\hline \multirow[t]{7}{*}{$\mathrm{Mo}(1)-\mathrm{S}(2)$} & $\pi^{*}$ & 25.74 & 0.79 & 0.49 & 98.72 & & 17.92 & 0.82 & 98.94 & 0.24 \\
\hline & & & & & & $\mathrm{S}(4)$ & 7.54 & 0.78 & 97.80 & 1.41 \\
\hline & & & & & & $\mathrm{S}(5)$ & 6.74 & 0.18 & 98.39 & 1.43 \\
\hline & & & & & & $\mathrm{C}(10)$ & 17.71 & 0.12 & 99.79 & 0.09 \\
\hline & & & & & & $\mathrm{C}(11)$ & 17.34 & 0.36 & 99.55 & 0.09 \\
\hline & & & & & & $\mathrm{O}(13)$ & 2.14 & 19.76 & 80.21 & 0.03 \\
\hline & & & & & & $\mathrm{C}(6)$ & $<2$ & & & \\
\hline $\mathrm{W}(1)-\mathrm{S}(3)$ & $\sigma$ & 22.56 & 16.64 & 1.05 & 82.31 & & 74.07 & 18.88 & 80.96 & 0.16 \\
\hline \multirow[t]{3}{*}{$\mathrm{W}(1)-\mathrm{S}(3)$} & $\pi$ & 30.21 & 0.22 & 0.25 & 99.53 & & 63.98 & 0.21 & 99.70 & 0.09 \\
\hline & & & & & & $\mathrm{C}(7)$ & 2.17 & 0.25 & 98.25 & 1.50 \\
\hline & & & & & & $\mathrm{C}(6)$ & $<2 \%$ & & & \\
\hline $\mathrm{S}(2)-\mathrm{C}(6)$ & $\sigma$ & 45.36 & 21.84 & 77.60 & 0.57 & & 53.47 & 27.15 & 72.70 & 0.15 \\
\hline $\mathrm{S}(3)-\mathrm{C}(7)$ & $\sigma$ & 45.10 & 21.02 & 78.41 & 0.57 & & 53.60 & 25.45 & 74.41 & 0.14 \\
\hline $\mathrm{C}(6)-\mathrm{C}(7)$ & $\sigma$ & 49.14 & 35.29 & 64.68 & 0.04 & & 50.07 & 32.96 & 67.00 & 0.04 \\
\hline \multirow[t]{7}{*}{$\mathrm{C}(6)-\mathrm{C}(7)$} & $\pi$ & 42.86 & 0.02 & 99.94 & 0.04 & & 41.39 & 0.02 & 99.94 & 0.04 \\
\hline & & & & & & $\operatorname{Mo}(1)$ & 2.63 & 2.39 & 1.23 & 96.38 \\
\hline & & & & & & $\mathrm{S}(2)$ & 2.54 & 0.13 & 94.28 & 5.60 \\
\hline & & & & & & $\mathrm{S}(3)$ & 2.23 & 0.09 & 94.53 & 5.38 \\
\hline & & & & & & $\mathrm{C}(10)$ & $<2$ & & & \\
\hline & & & & & & $\mathrm{C}(11)$ & $<2$ & & & \\
\hline & & & & & & $\mathrm{C}(15)$ & $<2$ & & & \\
\hline $\mathrm{S}(4)-\mathrm{C}(13)$ & $\sigma$ & 44.43 & 20.27 & 79.13 & 0.60 & & 54.38 & 27.36 & 72.49 & 0.15 \\
\hline \multirow[t]{3}{*}{$\mathrm{S}(4)-\mathrm{C}(13)$} & $\pi$ & 66.00 & 0.00 & 99.82 & 0.18 & & 25.83 & 0.02 & 99.85 & 0.13 \\
\hline & & & & & & $\operatorname{Mo}(1)$ & 2.84 & 1.97 & 0.66 & 97.37 \\
\hline & & & & & & $\mathrm{C}(10)$ & $<2$ & & & \\
\hline $\mathrm{S}(5)-\mathrm{C}(12)$ & $\sigma$ & 44.38 & 19.45 & 79.95 & 0.60 & & 54.34 & 25.65 & 74.21 & 0.14 \\
\hline \multirow[t]{3}{*}{$\mathrm{S}(5)-\mathrm{C}(12)$} & $\pi$ & 67.31 & 0.00 & 99.85 & 0.15 & & 23.61 & 0.10 & 99.79 & 0.11 \\
\hline & & & & & & $\operatorname{Mo}(1)$ & 3.26 & 1.80 & 0.91 & 97.29 \\
\hline & & & & & & $\mathrm{C}(11)$ & $<2$ & & & \\
\hline $\mathrm{C}(12)-\mathrm{C}(13)$ & $\sigma$ & 50.102 & 33.24 & 66.72 & 0.04 & & 49.08 & 35.39 & 64.56 & 0.04 \\
\hline $\mathrm{C}(12)-\mathrm{C}(13)$ & - & . & - & - & - & - & - & - & - & - \\
\hline
\end{tabular}

${ }^{a}$ Die Spalte "B" beinhaltet wechselwirkende Nachbaratome, die nicht direkt zur Bindung gehören. 
Tabelle 2.16: Bindungsparameter der M-S-Doppelbindungen $\left[\mathrm{M}^{V I} \mathrm{O}(\mathrm{fdt})_{2}\right](\mathrm{M}=\mathrm{Mo}$, W)

\begin{tabular}{|c|c|c|c|c|c|c|c|c|c|c|}
\hline \multirow[t]{2}{*}{$\mathrm{A}-\mathrm{B}$} & \multirow[t]{2}{*}{ Тур } & \multirow[t]{2}{*}{$\% \mathrm{~A}$} & \multicolumn{3}{|c|}{ Hybridisierung } & \multirow[t]{2}{*}{$\mathrm{B}^{a}$} & \multirow[t]{2}{*}{$\% \mathrm{~B}$} & \multicolumn{3}{|c|}{ Hybridisierung } \\
\hline & & & $\mathrm{s}$ & $\mathrm{p}$ & $\mathrm{d}$ & & & $\mathrm{s}$ & $\mathrm{p}$ & $\mathrm{d}$ \\
\hline $\mathrm{Mo}(1)-\mathrm{S}(2)$ & $\bar{\sigma}$ & 24.43 & 11.38 & 0.67 & 87.95 & & 72.45 & 15.90 & 83.95 & 0.15 \\
\hline $\mathrm{Mo}(1)-\mathrm{S}(2)$ & $\pi$ & 45.68 & 0.05 & 0.08 & 99.87 & & 48.29 & 0.14 & 99.73 & 0.12 \\
\hline \multirow[t]{6}{*}{$\mathrm{Mo}(1)-\mathrm{S}(2)$} & $\pi^{*}$ & 24.78 & 0.96 & 0.48 & 98.56 & & 16.28 & 0.96 & 98.78 & 0.25 \\
\hline & & & & & & $\mathrm{S}(4)$ & 8.10 & 0.61 & 98.07 & 1.33 \\
\hline & & & & & & $\mathrm{S}(5)$ & 6.90 & 0.19 & 98.40 & 1.40 \\
\hline & & & & & & $\mathrm{C}(11)$ & 18.80 & 0.15 & 99.77 & 0.09 \\
\hline & & & & & & $\mathrm{C}(12)$ & 18.27 & 0.44 & 99.46 & 0.09 \\
\hline & & & & & & $\mathrm{O}(15)$ & $<2$ & & & \\
\hline $\mathrm{W}(1)-\mathrm{S}(2)$ & $\sigma$ & 24.48 & 16.17 & 0.88 & 82.95 & & 72.73 & 18.26 & 81.57 & 0.17 \\
\hline \multirow[t]{3}{*}{$\mathrm{W}(1)-\mathrm{S}(2)$} & $\pi$ & 31.53 & 0.16 & 0.21 & 99.63 & & 61.87 & 0.32 & 99.58 & 0.10 \\
\hline & & & & & & $\mathrm{C}(6)$ & 2.16 & 0.23 & 98.38 & 1.39 \\
\hline & & & & & & $\mathrm{C}(7)$ & 2.02 & 0.96 & 98.94 & 0.09 \\
\hline $\mathrm{S}(2)-\mathrm{C}(6)$ & $\sigma$ & 45.18 & 21.71 & 77.71 & 0.58 & & 53.63 & 26.97 & 72.89 & 0.15 \\
\hline $\mathrm{S}(3)-\mathrm{C}(7)$ & $\sigma$ & 44.94 & 20.92 & 78.51 & 0.57 & & 53.73 & 25.31 & 74.55 & 0.14 \\
\hline $\mathrm{C}(6)-\mathrm{C}(7)$ & $\sigma$ & 49.14 & 35.03 & 64.93 & 0.04 & & 50.02 & 33.15 & 66.81 & 0.04 \\
\hline \multirow[t]{7}{*}{$\mathrm{C}(6)-\mathrm{C}(7)$} & $\pi$ & 43.34 & 0.02 & 99.95 & 0.04 & & 41.23 & 0.15 & 99.82 & 0.04 \\
\hline & & & & & & $\operatorname{Mo}(1)$ & 2.76 & 2.40 & 1.03 & 96.57 \\
\hline & & & & & & $\mathrm{S}(2)$ & 2.60 & 0.13 & 94.36 & 5.51 \\
\hline & & & & & & $\mathrm{S}(3)$ & 2.15 & 0.08 & 94.42 & 5.49 \\
\hline & & & & & & $\mathrm{C}(11)$ & $<2$ & & & \\
\hline & & & & & & $\mathrm{C}(12)$ & $<2$ & & & \\
\hline & & & & & & $\mathrm{C}(17)$ & $<2$ & & & \\
\hline $\mathrm{S}(4)-\mathrm{C}(13)$ & $\sigma$ & 44.11 & 19.98 & 79.41 & 0.62 & & 54.67 & 27.26 & 72.60 & 0.14 \\
\hline \multirow[t]{3}{*}{$\mathrm{S}(4)-\mathrm{C}(13)$} & $\pi$ & 65.27 & 0.00 & 99.81 & 0.18 & & 26.25 & 0.03 & 99.86 & 0.11 \\
\hline & & & & & & $\operatorname{Mo}(1)$ & 2.89 & 1.79 & 0.71 & 97.51 \\
\hline & & & & & & $\mathrm{C}(11)$ & $<2$ & & & \\
\hline $\mathrm{S}(5)-\mathrm{C}(12)$ & $\sigma$ & 44.20 & 19.17 & 80.22 & 0.61 & & 54.45 & 25.46 & 74.41 & 0.14 \\
\hline \multirow[t]{3}{*}{$\mathrm{S}(5)-\mathrm{C}(12)$} & $\pi$ & 67.85 & 0.00 & 99.85 & 0.15 & & 23.10 & 0.14 & 99.75 & 0.11 \\
\hline & & & & & & $\operatorname{Mo}(1)$ & 3.35 & 2.22 & 0.95 & 96.83 \\
\hline & & & & & & $\mathrm{C}(12)$ & $<2$ & & & \\
\hline $\mathrm{C}(12)-\mathrm{C}(13)$ & $\sigma$ & 50.06 & 33.50 & 66.45 & 0.04 & & 49.07 & 35.09 & 64.87 & 0.04 \\
\hline $\mathrm{C}(12)-\mathrm{C}(13)$ & - & - & - & - & - & - & - & - & - & - \\
\hline
\end{tabular}

${ }^{a}$ Die Spalte "B" beinhaltet wechselwirkende Nachbaratome, die nicht direkt zur Bindung gehören. 
Tabelle 2.17: Besetzungen und Bindungsordungen BO der M(1)-S(2)-Bindung

\begin{tabular}{ccccc}
\hline $\mathrm{Mo}(1)-\mathrm{S}(2)$ & $\mathbf{3 c}$ & 4c & 5c & 6c \\
\hline$\sigma$ & 1.798 & 1.814 & 1.794 & 1.813 \\
$\pi$ & 1.869 & 1.806 & 1.866 & 1.846 \\
$\pi^{*}$ & 1.096 & & 1.047 & \\
$\mathrm{BO}$ & 1.3 & 1.8 & 1.3 & 1.8
\end{tabular}

Metall sowie an die angrenzenden C-C-Atome gebunden ist, kommt in der Form nur in den OS V und IV vor. In Oxidationsstufe VI erfolgt eine drastische Änderung im Bindungsverhalten, sowohl bei den Chromanyl- als auch bei den Flavanylverbindungen. Da das freie Elektronenpaar am Metall wegfällt, werden die freien Elektronenpaare des Schwefels herangezogen. So wird bei den Chromanylkomplexen das freie Elektronenpaar von $\mathrm{S}(2)$ in der Molybdänverbindung und von $\mathrm{S}(3)$ in der Wolframverbindung in die sich bildende Schwefel-Metall-Doppelbindung involviert. Bei den Flavanylkomplexen handelt es sich bei beiden Metallen um das freie Elektronenpaar vom Atom S(2). Der $\pi$-Charakter dieser zweiten Bindung beträgt nahezu 100\%. Die Anteile der einzelnen Bindungspartner betragen bei den Wolframverbindungen ähnlich zur normalen Metall-Schwefel- $\sigma$-Bindung von etwa 30\% Metall und 65\% Schwefel, bei den Molybdänverbindungen liegen die Anteile bei etwa $47 \%$ jeweils für Metall und Schwefel. Bei den Molybdänverbindungen der Chromanyl- und Flavanylsysteme sind zusätzlich antibindende Anteile enthalten, deren abstoßende Anteile aus der jeweils gegenüberliegenden Dithioleneinheit stammen.

Tabelle 2.17 verdeutlicht das Ausmaß der bindungsabschwächenden Wirkung anhand der aus den bindenen und antibindenden Elektronen berechneten Bindungsordnungen, wobei hier für die Bindungsordnung gilt

$$
B O=\frac{1}{2}\left(e_{\text {bindend }}^{-}-e_{\text {antibindend }}^{-}\right) .
$$

Die BO von 1.3 für die Mo-Verbindungen ist zwar nach wie vor eine stabile Bindung, jedoch ist die an den W-Verbindungen mit 1.8 fast als Doppelbindung vorlie- 
Abbildung 2.13: Lewisstrukturen der Verbindungen $\left[\mathrm{MO}^{V I}(\mathrm{cdt})_{2}\right]$ und $\left[\mathrm{MO}^{V I}(\mathrm{fdt})_{2}\right]$, $\mathrm{M}=\mathrm{Mo}, \mathrm{W}$
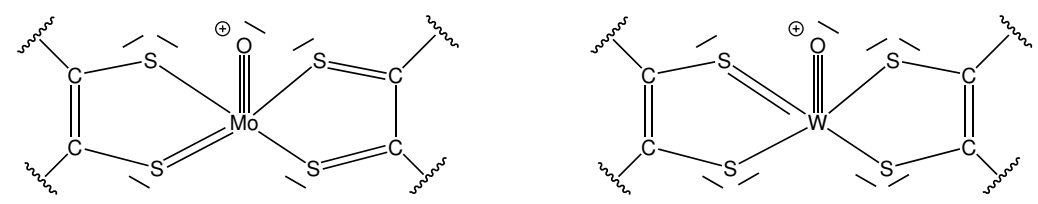

gende Bindung wesentlich stärker.

Die aus der gegenüberliegenden Dithioleneinheit abgegebenen Elektronen werden vom Wolfram scheinbar stärker kompensiert; möglich ist dies aufgrund der höheren Kernladungszahl verbunden mit der Kontraktion der inneren Schale. Wolfram ist damit in der Lage, eine derartige Konfiguration zu stabilisieren, die im Fall des Molybdäns zu repulsiven Effekten einhergehend mit antibindenen Wechselwirkungen führt.

Eine weitere Besonderheit an den Verbindungen in OS VI zeigen die Lewisstrukturen in Bezug auf die Dithioleneinheit, wie in Abbildung 2.13 gezeigt.

Sowohl bei den Chromanyl- als auch den Flavanyl-Verbindungen zeigen die MolybdänKomplexe ein anderes Verhalten als die Wolfram-Komplexe. Eines der freien Elektronenpaare der Schwefel-Atome S(4) und S(5) klappt in die Bindung des sie benachbarten C-Atoms und bildet auf diese Art und Weise eine S-C-Doppelbindung aus während die ursprünglich doppelt gebundenen C-Atome $\mathrm{C}(10)$ und $\mathrm{C}(11)$ jetzt nur noch einfach gebunden vorliegen.

Möglich ist diese Situation durch den mit der C-C-Doppelbindung in Konjugation stehenden Benzolring, welcher als starker $\pi$-Eletrondonator fungiert. Der durch das Metall an Elektronen verarmte Schwefel bildet zur Stabilisierung eine Doppelbindung mit dem angrenzenden Kohlenstoff, welcher den aufgrund dieser Situation hervorgerufenen Elektronenüberschuss an die gegenüberliegende M-S(2)-Doppelbindung abgibt, deren p-Orbitale in umgekehrter Phase stehen, woraus eine antibindende Wechselwirkung resultiert. Die C-S-Doppelbindung ist durch das $\pi$-elektronenreiche Rückgrat 
Abbildung 2.14: Antibindende Wechselwirkung M(1)-S(2)

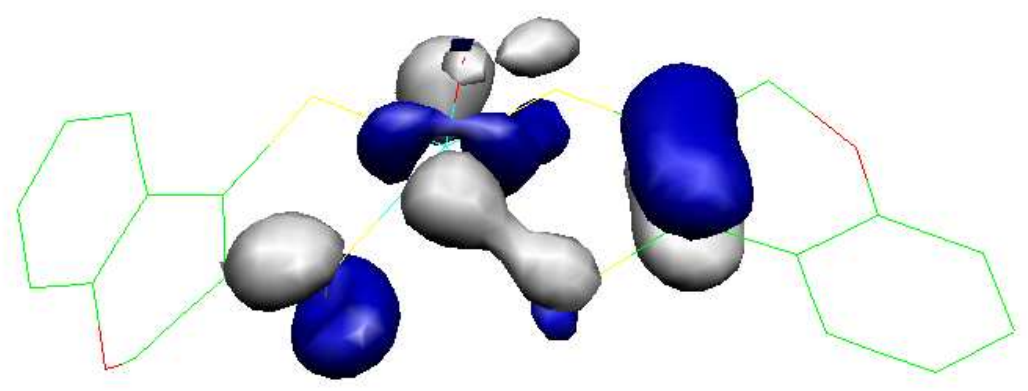

ebenfalls durch Konjugation stabilisiert, was auch die Werte aus der Bindungsanalyse zeigen. Neben recht hohen Anteilen der gegenüberliegenden Schwefel-Atome S(4) und S(5) mit $7 \%$ tragen die Atome $\mathrm{C}(10)$ und $\mathrm{C}(11)$ mit je $17 \%$ stark zu dieser Bindung bei (Flavanylverbindung S(4) und S(5) mit 8 und 7\%, C(11)/C(12) mit 19 und 18\%). Auch in den anderen OS IV und V finden sich Beiträge aus dem aromatischen Ring in der C-C-Doppelbindung mit wechselnden Beiträgen von bis zu $3 \%$.

Die Wolframkomplexe in OS VI dagegen bilden nur eine W-S-Doppelbindung aus, im Fall der Chromanylverbindungen W(1)-S(3) und bei den Flavanylverbindungen W(1)-S(2). Die Anteile der Bindungspartner betragen 30\% Metall und 64\% Schwefel bei der Chromanylverbindung und 31\% Metall vs. $62 \%$ Schwefel bei der Flavanylverbindung. Zusätzlich beteiligen sich die C-Atome $\mathrm{C}(6)$ und $\mathrm{C}(7)$ der benachbarten C-C-Doppelbindung mit ca. $2 \%$ an dieser Bindung. Wolfram kann also die OS VI trotz der ungünstigen elektronischen Situation wesentlich besser stabilisieren, da es aufgrund der höheren KLZ und relativistischer Effekte die durch zu starke Konjugation des Aromaten anfallende elektronische Dichte besser abfangen kann.

Die Orbitalenergien der höchsten besetzten Orbitale liegen in einer den Pyranylkomplexen ähnlichen Größenordnung, wenn auch marginal niedriger. 
Tabelle 2.18: Orbitalenergien $\varepsilon$ der Orbitale 110 - 115 [a.u.] von $\left[\mathrm{MO}(\mathrm{cdt})_{2}\right]^{z-}$ und (150-155) und $\left[\mathrm{MO}(\mathrm{fdt})_{2}\right]^{z-}, \mathrm{M}=\mathrm{Mo}, \mathrm{W} ; \mathrm{z}=-2,-1,0$

\begin{tabular}{cccccccc}
\hline & & $\mathbf{3 a}$ & $\mathbf{3 b}$ & $\mathbf{3 c}$ & $\mathbf{4 a}$ & $\mathbf{4 b}$ & $\mathbf{4 c}$ \\
\hline & 110 & 0.03 & -0.13 & -0.24 & 0.02 & -0.12 & -0.23 \\
& 111 & 0.04 & -0.10 & -0.21 & 0.04 & -0.10 & -0.21 \\
HOMO & 112 & 0.05 & -0.08 & -0.16 & 0.06 & -0.08 & -0.15 \\
LUMO & 113 & 0.16 & 0.04 & -0.09 & 0.16 & 0.05 & -0.07 \\
& 114 & 0.16 & 0.05 & -0.07 & 0.16 & 0.05 & -0.07 \\
& 115 & 0.18 & 0.05 & -0.07 & 0.18 & 0.08 & -0.06 \\
$\Delta \varepsilon(113-112)$ & & 0.11 & 0.12 & 0.08 & 0.10 & 0.13 & 0.08 \\
& & & & & & & \\
\hline & & $\mathbf{5 a}$ & $\mathbf{5 b}$ & $\mathbf{5 c}$ & $\mathbf{6 a}$ & $\mathbf{6 b}$ & $\mathbf{6 c}$ \\
\hline \multirow{4}{*}{ HOMO } & 150 & 0.02 & -0.13 & -0.23 & 0.01 & -0.12 & -0.23 \\
& 151 & 0.04 & -0.10 & -0.21 & 0.04 & -0.11 & -0.21 \\
& 152 & 0.04 & -0.08 & -0.16 & 0.05 & -0.08 & -0.15 \\
& 154 & 0.14 & 0.04 & -0.08 & 0.14 & 0.04 & -0.07 \\
& 155 & 0.14 & 0.04 & -0.07 & 0.14 & 0.05 & -0.06 \\
$\Delta \varepsilon(153-152)$ & & 0.10 & 0.12 & 0.08 & 0.09 & 0.13 & 0.08
\end{tabular}

Auch hier ist der Trend zu beobachten, dass die Energie mit zunehmender OS im Bereich von 0.2 a.u. stark abgesenkt wird. Wie in Tabelle 2.18 gezeigt, erfolgt die Abnahme gleichmäßig über beide Oxidationsschritte. Der Wechsel des Metalls hat wiederum keinen großen Einfluss auf die energetische Lage der Orbitale, ebensowenig wie der Wechsel des Liganden, dessen konstitutionelle Unterschiede die Metallumgebung nicht beeinflussen. Die HOMO-LUMO-Lücke wird mit zunehmender OS kleiner, bei beiden Systemen um ähnliche Werte von 0.03 Hartree.

Deutliche Unterschiede zwischen den beiden Systemen der Chromanyl- und Flavanylverbindungen, sowie den beiden Metallen Mo und W zeigen sich allerdings in der räumlichen Anordung der HOMOs.

Abbildung 2.15 zeigt die höchsten besetzten Orbitale der Verbindungen 3a (das HOMO sowie das HOMO-1). Die entsprechenden Orbitalkoeffizienten aller Verbin- 
Abbildung 2.15: Grenzorbitale der Verbindung 3a

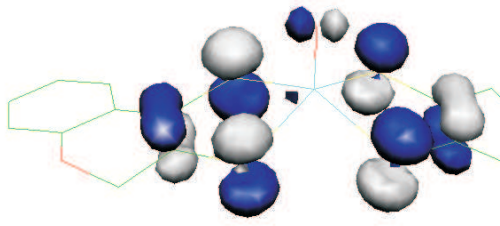

HOMO

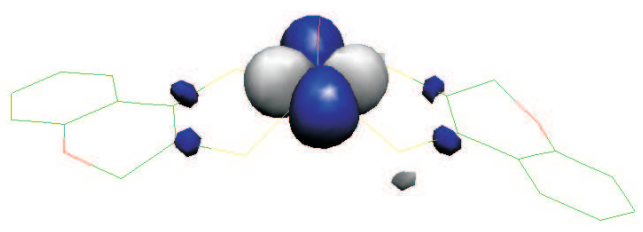

HOMO-1

dungen sind in Tabelle 2.19 dargestellt.

Bei den Chromanylverbindungen zeigt sich bei den Molybdänverbindungen eine Abnormalität in der OS IV, bei der das HOMO überwiegend ligandenzentriert vorliegt. Der Metallanteil beträgt hier nur $9.9 \%$ des $\mathrm{d}_{x^{2} y^{2}}$-Orbitals und zwar in umgekehrter Phase zu den restlichen Komplexen. Der Anteil der Ligandenatome ist nur unwesentlich höher und liegt bei Werten von etwa 10\% pro Atom. Das normalerweise als höchstes besetztes Orbital vorliegende $\mathrm{d}_{x^{2} y^{2}}$-Orbital des Metalls ist hierbei nahezu entartet (Orbitalenergien: 0.03657 HOMO-1 und 0.03763 HOMO) mit dem darunterliegenden HOMO-1, welches ein ligandenzentriertes $\mathrm{MO}$ aus p-Orbitalen der Schwefel-Atome ist. In diesem Fall kommt es zu einer Orbitalinversion, so dass das HOMO-1 und das HOMO vertauscht vorliegen.

In OS V steigt der Metallanteil auf 25.8\% und in OS VI auf 39.9\%. Diese Werte sind wiederum niedriger als bei den vergleichbaren Wolframverbindungen, die mit einem Metallanteil von je $67.1 \%$ in OS IV, $33.4 \%$ in OS V und mit $48.2 \%$ in OS VI deutlich größer sind. Die Verteilung der Elektronendichte bei den restlichen Atomen ist nur von geringen Schwankungen betroffen und zeigt lediglich bei der Wolframverbindung in OS IV eine erwähnenswerte Abweichung. Da sich hier die meiste Elektronendichte am Metall befindet, verbleibt nur sehr wenig bei den Nachbaratomen. So betragen die Orbitalkoeffizienten der Schwefelatome nur noch durchschnittlich 2\%, die der CAtome nur 5 bis 7\%. Auch ist die räumliche Ausrichtung der Schwefel-Atome nicht 
Tabelle 2.19: Zusammensetzung der redoxaktiven Orbitale (112 der Chromanylverbindungen und 152 der Flavanylverbindungen)

$$
\left[\mathrm{MO}(\mathrm{cdt})_{2}\right]^{z-}(\mathrm{M}=\mathrm{Mo}, \mathrm{W} ; \mathrm{z}=-2,-1,0)
$$

\begin{tabular}{cccccc}
\hline & $\mathrm{M}\left(\mathrm{d}_{x^{2} y^{2}}\right)$ & $\mathrm{S}(2)\left(\mathrm{p}_{z}\right)$ & $\mathrm{S}(3)\left(\mathrm{p}_{z}\right)$ & $\mathrm{S}(4)\left(\mathrm{p}_{z}\right)$ & $\mathrm{S}(5)\left(\mathrm{p}_{z}\right)$ \\
\hline & & & & & \\
3a & -0.09977 & -0.09900 & -0.10777 & 0.10259 & 0.11013 \\
$\mathbf{3 b}$ & 0.25779 & -0.10007 & -0.09267 & 0.09389 & 0.08716 \\
$\mathbf{3 c}$ & 0.39926 & -0.09276 & -0.08702 & 0.08361 & 0.07505 \\
4a & 0.67122 & $0.02264\left(\mathrm{p}_{y}\right)$ & -0.02226 & -0.02079 & 0.02428 \\
4b & 0.33420 & -0.09533 & -0.09613 & 0.08485 & 0.08566 \\
4c & 0.48231 & -0.08499 & -0.08002 & 0.07677 & 0.07021 \\
& & & & & \\
\hline & $\mathrm{C}(6)\left(\mathrm{p}_{z}\right)$ & $\mathrm{C}(7)\left(\mathrm{p}_{z}\right)$ & $\mathrm{C}(12)\left(\mathrm{p}_{z}\right)$ & $\mathrm{C}(13)\left(\mathrm{p}_{z}\right)$ & $\mathrm{O}(17)\left(\mathrm{p}_{x}\right)$ \\
\hline & & & & & \\
3a & 0.11271 & 0.11391 & -0.10771 & -0.10751 & 0.05949 \\
3b & 0.10506 & 0.09319 & -0.11893 & -0.12638 & -0.05687 \\
$\mathbf{3 c}$ & 0.09081 & 0.08176 & -0.11102 & -0.11953 & -0.04200 \\
4a & $0.07056\left(\mathrm{p}_{x}\right)$ & 0.05312 & -0.05338 & -0.07186 & $0.01145\left(\mathrm{p}_{z}\right)$ \\
4b & 0.09930 & 0.10054 & -0.11366 & -0.11634 & 0.07237 \\
4c & 0.06880 & 0.06388 & -0.09963 & -0.10603 & -0.04911
\end{tabular}

$$
\left[\mathrm{MO}(\mathrm{fdt})_{2}\right]^{z-}(\mathrm{M}=\mathrm{Mo}, \mathrm{W} ; \mathrm{z}=-2,-1,0)
$$

\begin{tabular}{cccccc}
\hline & $\mathrm{M}\left(\mathrm{d}_{x^{2} y^{2}}\right)$ & $\mathrm{S}(2)\left(\mathrm{p}_{z}\right)$ & $\mathrm{S}(3)\left(\mathrm{p}_{z}\right)$ & $\mathrm{S}(4)\left(\mathrm{p}_{z}\right)$ & $\mathrm{S}(5)\left(\mathrm{p}_{z}\right)$ \\
\hline $\mathbf{5 a}$ & 0.66777 & -0.03869 & -0.04369 & 0.06434 & 0.06211 \\
$\mathbf{5 b}$ & 0.27438 & 0.10731 & 0.10202 & -0.09026 & -0.08440 \\
$\mathbf{5 c}$ & 0.41607 & 0.09565 & 0.08765 & -0.08488 & -0.07294 \\
$\mathbf{6 a}$ & 0.73043 & $0.02248\left(\mathrm{p}_{y}\right)$ & -0.02503 & -0.02024 & 0.02591 \\
$\mathbf{6 b}$ & 0.36466 & 0.10057 & 0.09194 & -0.09056 & -0.08409 \\
$\mathbf{6 c}$ & 0.50052 & 0.08700 & 0.07941 & -0.07785 & -0.06779 \\
& & & & & \\
\hline & $\mathrm{C}(6)\left(\mathrm{p}_{z}\right)$ & $\mathrm{C}(7)\left(\mathrm{p}_{z}\right)$ & $\mathrm{C}(12)\left(\mathrm{p}_{z}\right)$ & $\mathrm{C}(13)\left(\mathrm{p}_{z}\right)$ & $\mathrm{O}(15)\left(\mathrm{p}_{x}\right)$ \\
\hline
\end{tabular}

$\begin{array}{cccccc}\mathbf{5 a} & 0.07074 & 0.06559 & -0.04344 & -0.05928 & -0.01231\left(\mathrm{p}_{z}\right) \\ \mathbf{5 b} & -0.10662 & -0.11538 & 0.11837 & 0.11432 & 0.07841 \\ \mathbf{5 c} & -0.08627 & -0.08356 & 0.11027 & 0.11335 & 0.04137 \\ \mathbf{6 a} & -0.04896 & 0.04067 & -0.04101 & 0.05159 & 0.00221 \\ \mathbf{6 b} & -0.09710 & -0.09261 & 0.10708 & 0.11589 & -0.05547 \\ \mathbf{6 c} & -0.06198 & -0.06449 & 0.10014 & 0.10101 & -0.01978\end{array}$


mehr von $\mathrm{p}_{z^{-}}$sondern von $\mathrm{p}_{y^{-}}$-Gestalt, was für diesen Fall eine bessere Überlappung bietet.

Die Orbitalkoeffizienten der Flavanylverbindungen weisen den gleichen Trend wie die Chromanylverbindungen auf. Die Metallanteile sind jedoch um bis zu 5\% deutlich höher, während es bei den restlichen Schwefel- und Kohlenstoff-Atomen keine wesentlichen Unterschiede gibt. Der Molybdän-Komplex in OS IV weist einen Metall-Anteil von $66.8 \%$ auf und liegt damit im Bereich des analogen Wolframchromanyl-Komplexes mit 67.1\%. Auch ist hier der Anteil der Schwefel-Atome im Mittel geringer wenn auch nicht in dem Maße wie bei den Wolfram-Analoga. Diese Situation wird durch die in diesen Molekülen auftretende Konjugation ermöglicht, durch die ein enorm hohes Maß an elektronischer Dichte an das Metall weitergeleitet wird.

Für eventuell vorliegende Wechselwirkungen der Schwefel-Atome untereinander, wurde eine NBO-Analyse der betreffenden Bindungen (S(2)-S(3) und S(4)-S(5)) durchgeführt. Die Besetzungen der bindenden und antibindenden Orbitale sowie die daraus ermittelten Bindungsordnungen sind in Tabelle 2.20 dargestellt.

Beide Systeme weisen untereinander ähnliche Werte auf, die sich jedoch von den Werten der Pyranylverbindungen unterscheiden. Sowohl für die Molybdän- als auch die Wolfram-Komplexe ergeben sich in OS IV bindende Wechselwirkungen, die für die Molybdänverbindungen marginal größer ausfallen (0.08/0.07). Diese recht schwache Bindung ist in den Liganden auf beiden Seiten vorhanden. Wiederum ist in OS V in keinem Fall eine derartige Wechselwirkung zu finden. In der OS VI findet sich bei den Molybdänverbindungen beider Ligandensysteme eine höhere Wechselwirkung mit BO von 0.16 auf der Seite des nicht gefalteten Liganden und 0.15 auf der Seite des gefalteten Liganden, dagegen zeigen die Wolframverbindungen dieses Phänomen nur auf der Seite des gefalteten Liganden mit einer BO von 0.12. Der Ligand auf der gegenüberliegenden Seite jedoch führt mit einer höheren Besetzung des antibindenden 
Tabelle 2.20: Besetzungen und Bindungsordnungen BO benachbarter S-Atome

\begin{tabular}{|c|c|c|c|c|c|}
\hline & Besetzung & $\mathrm{BO}$ & & Besetzung & $\mathrm{BO}$ \\
\hline $\mathbf{3 a}$ & & & $5 a$ & & \\
\hline $\mathrm{S}(2)-\mathrm{S}(3)$ & 1.80 & 0.08 & $\mathrm{~S}(2)-\mathrm{S}(3)$ & 1.80 & 0.08 \\
\hline $\mathrm{S}(2)-\mathrm{S}(3) *$ & 1.65 & & $\mathrm{~S}(2)-\mathrm{S}(3)^{*}$ & 1.64 & \\
\hline $\mathrm{S}(4)-\mathrm{S}(5)$ & 1.80 & 0.08 & $\mathrm{~S}(4)-\mathrm{S}(5)$ & 1.80 & 0.08 \\
\hline $\mathrm{S}(4)-\mathrm{S}(5)^{*}$ & 1.65 & & $\mathrm{~S}(4)-\mathrm{S}(5)^{*}$ & 1.64 & \\
\hline $3 c$ & & & $5 c$ & & \\
\hline $\mathrm{S}(2)-\mathrm{S}(3)$ & 1.67 & 0.16 & $\mathrm{~S}(2)-\mathrm{S}(3)$ & 1.67 & 0.16 \\
\hline $\mathrm{S}(2)-\mathrm{S}(3)^{*}$ & 1.34 & & $\mathrm{~S}(2)-\mathrm{S}(3)^{*}$ & 1.35 & \\
\hline $\mathrm{S}(4)-\mathrm{S}(5)$ & 1.63 & 0.15 & $\mathrm{~S}(4)-\mathrm{S}(5)$ & 1.64 & 0.15 \\
\hline $\mathrm{S}(4)-\mathrm{S}(5) *$ & 1.33 & & $\mathrm{~S}(4)-\mathrm{S}(5)^{*}$ & 1.34 & \\
\hline $4 a$ & & & $6 a$ & & \\
\hline $\mathrm{S}(2)-\mathrm{S}(3)$ & 1.78 & 0.07 & $\mathrm{~S}(2)-\mathrm{S}(3)$ & 1.78 & 0.07 \\
\hline $\mathrm{S}(2)-\mathrm{S}(3)^{*}$ & 1.65 & & $\mathrm{~S}(2)-\mathrm{S}(3)^{*}$ & 1.65 & \\
\hline $\mathrm{S}(4)-\mathrm{S}(5)$ & 1.79 & 0.07 & $\mathrm{~S}(4)-\mathrm{S}(5)$ & 1.79 & 0.07 \\
\hline $\mathrm{S}(4)-\mathrm{S}(5) *$ & 1.66 & & $\mathrm{~S}(4)-\mathrm{S}(5)^{*}$ & 1.65 & \\
\hline $4 c$ & & & $6 c$ & & \\
\hline $\mathrm{S}(2)-\mathrm{S}(3)$ & 1.68 & $(-0.16)$ & $\mathrm{S}(2)-\mathrm{S}(3)$ & 1.35 & $(-0.16)$ \\
\hline $\mathrm{S}(2)-\mathrm{S}(3)^{*}$ & 1.36 & & $\mathrm{~S}(2)-\mathrm{S}(3)^{*}$ & 1.68 & \\
\hline $\mathrm{S}(4)-\mathrm{S}(5)$ & 1.64 & 0.12 & $\mathrm{~S}(4)-\mathrm{S}(5)$ & 1.63 & 0.12 \\
\hline $\mathrm{S}(4)-\mathrm{S}(5)^{*}$ & 1.40 & & $\mathrm{~S}(4)-\mathrm{S}(5)^{*}$ & 1.40 & \\
\hline
\end{tabular}

Orbitals zu einer Antibindung gleicher Größenordnung.

\subsection{Die Systeme $\left[\mathrm{MO}(\mathrm{prz})_{2}\right]^{z-}$ und $\left[\mathrm{MO}(\mathrm{mpt})_{2}\right]^{z-}$}

\subsubsection{Ansatz zur Synthese eines trizyklischen molybdopterin- artigen Liganden}

Basierend auf der Syntheseroute nach Garner et al. sollte der Zugang zu Liganden, die neben dem Pyranring und der Dithioleneinheit auch über das Pteridingerüst verfügen, mittels geeigneter Ausgangsverbindungen möglich sein.

Ein entscheidender Schritt in der geplanten Synthese ist der von Kabbe [129] entwickelte Zugang zu Chromanonsystemen, die über Ringschlussreaktionen aus 3- 
Abbildung 2.16: Ringschlussreaktion von Chromanonsystemen

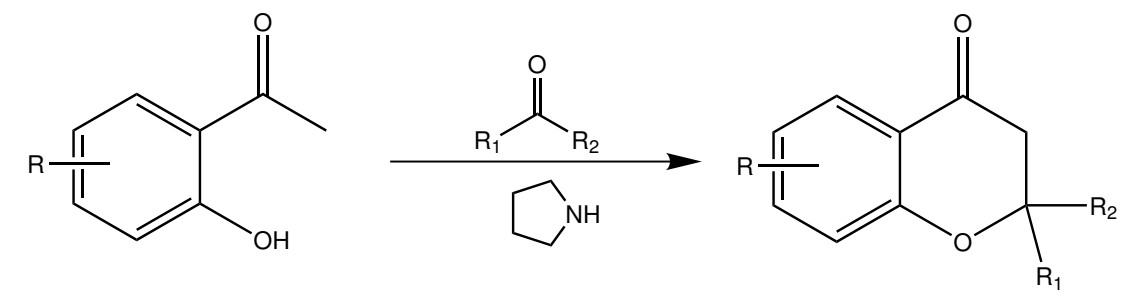

$\mathrm{R}=$ organischer Rest, $\mathrm{R}_{1}, \mathrm{R}_{2}=$ Alkyl-, Aryl-

Hydroxyketonen dargestellt werden (Abbildung 2.16).

Diese Möglichkeit des Aufbaus des Pyranrings innerhalb eines zyklischen Ketons, die auch weitere funktionelle Gruppen toleriert, erlaubt außerdem noch den Einbau einer Seitenkette am $\beta$-Keto-C-Atom. Wird die 3-Hydroxyketo-Funktionalität mit dem Pteridingerüst verknüpft, sollte eine Ringschlussreaktion nach Kabbe möglich sein. Die dafür erforderliche Ausgangsverbindung 22 ist ausgehend vom Aminouracil 20 über einen in der Literatur bekannten Weg [130] (Abbildung 2.17) zugänglich und konnte auf diesem Weg synthetisiert werden. Dafür wurde das Aminouracil mit Triphosgen zu der Zwischenstufe 21 umgesetzt, welche nach Reduktion mit $\mathrm{NaH}$ das gewünschte Pteridin, welches mit der 3-Hydroxyketo-Funktion verknüpft ist, ergibt. Diese Verbindung sollte sich nach der Syntheseroute von Kabbe zu Pteridin 23, welches mit einem Pyranon verbunden ist, umsetzen lassen.

Die folgende Umsetzung mit Aceton erwies sich jedoch als wenig geeignet, da die sich dann in 3-Keto-Stellung befindenden Methylgruppen $R_{1}$ und $R_{2}$, die nach einem $\mathrm{S}_{N} 2$-Mechanismus ablaufende weitere Umsetzung sterisch behindern.

Aufgrund des hohen Anschaffungspreises geeigneter Aldehyde und der geringen Reaktivität von 23 wurden die Arbeiten an dieser Route, trotz bis zu einem bestimmten Punkt vielversprechender Vorversuche mit Aceton, nicht weiter verfolgt.

Sollte eine genügend reaktive Verbindung 23 (mit $R_{1}$ und/oder $R_{2}=H$ ) synthetisiert werden können, würde diese die weitere Umsetzung nach der Syntheseroute von 
Abbildung 2.17: Aufbau eines Pteridin-Dithiocarbonats

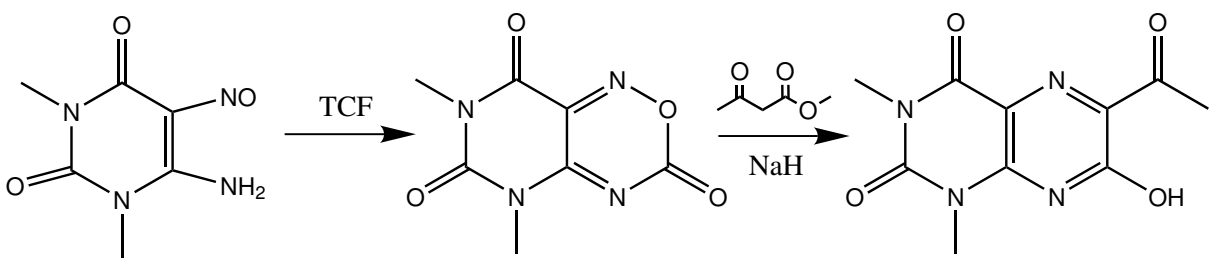

21

22<smiles></smiles>

24

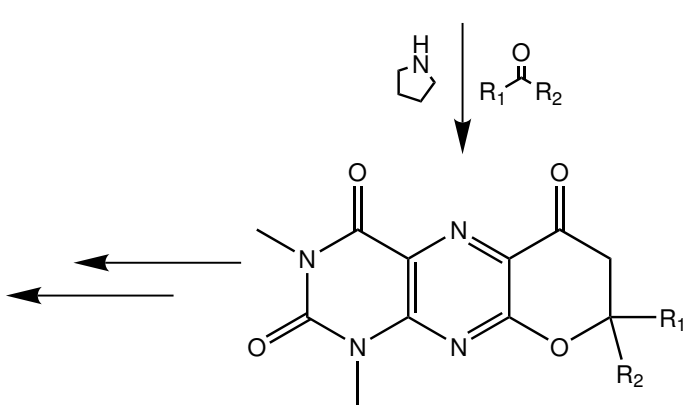

23

Garner zu dem Dithiocarbonat 24 erlauben, welches nun nach Umsetzung mit den entsprechenden Metallen zu Pteridindithiolenkomplexen führen sollte.

\subsubsection{Geometrische Strukturen}

Die Verbindungen des Pyrazins dienen insofern als Modellverbindungen als dass sie über zwei Ringe des Pyranopterin-Kofaktors verfügen: den Tetrahydropyran- und den folgenden Tetrahydropyrazinring. Anstelle des folgenden dritten Ringes erfolgt der Abschluss anhand einer C-C-Doppelbindung, die auch im Pyranopterin an dieser Stelle vorhanden ist. Die Konformation der beiden die Ringe verknüpfenden Stelle ist dem Pyranopterin nachempfunden, jeweils beide C-Atome liegen in der $R$-Konformation vor, so dass die beteiligten H-Atome in cis-Stellung zueinander liegen und der Pyrazinring in abgewinkelter Form vorliegt.

Der Pyranring liegt wiederum in Halbsesselkonformation vor und der abgewinkelte Pyrazinring besitzt eine nur leicht gestörte Planarität.

Analog ist die Geometrie der Modellkomplexe, die den gesamten Pyranopterinli- 
Abbildung 2.18: Strukturen der Verbindungen $\left[\mathrm{MO}(\mathrm{prz})_{2}\right]^{z-}$ und $\left[\mathrm{MO}(\mathrm{mpt})_{2}\right]^{z-}$

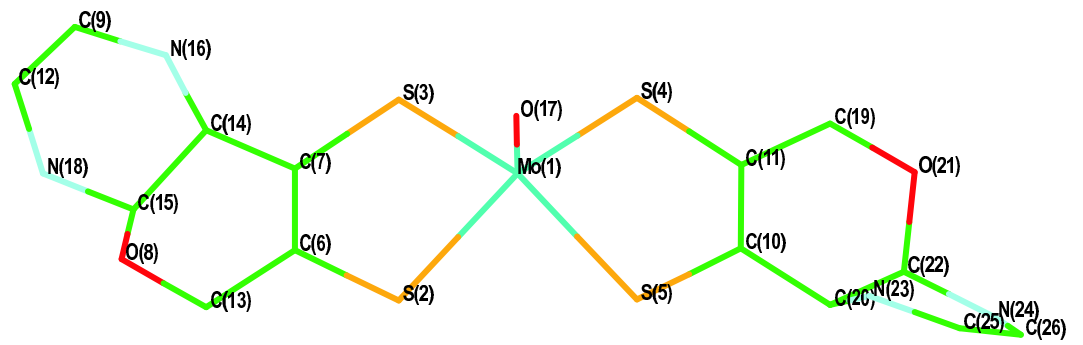

$\left[\mathrm{MO}(\mathrm{prz})_{2}\right]^{\mathrm{z}-}$
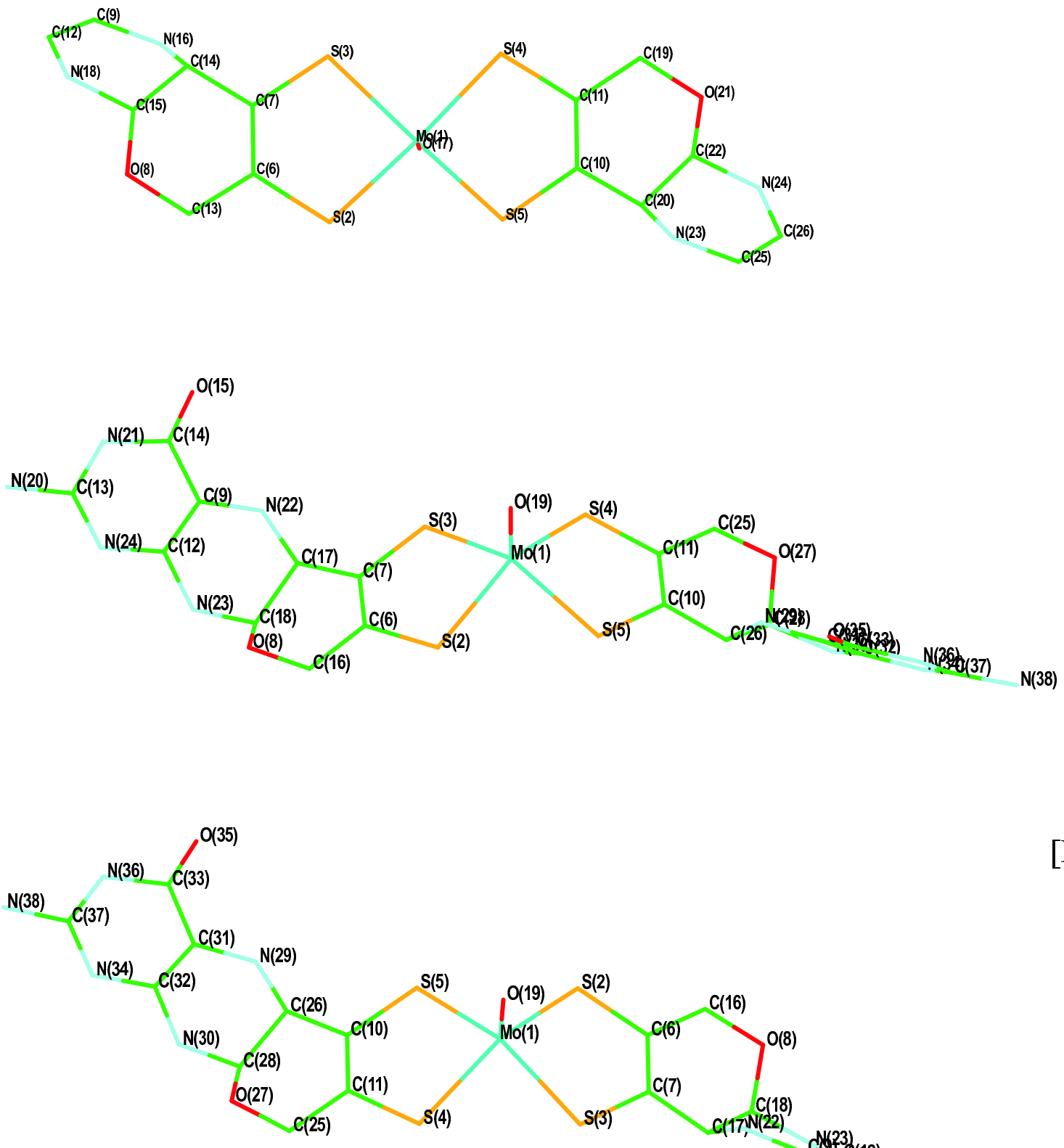
ganden enthalten, gewählt. Am Pyrazinring ist zusätzlich der dritte Aminopyrimidinonring über die C-C-Doppelbindung verknüpft und liegt bezogen auf diese und deren angrenzenden Stickstoff-Atome planar vor. In Anlehnung an die von R. Huber [131] aufgeklärte Struktur liegen die neben dem Keto-Sauerstoff liegenden Stickstoff-Atome $\mathrm{N}(21)$ und $\mathrm{N}(36)$ in protonierter Form vor. Abbildung 2.18 zeigt exemplarisch für die gesamte Elektronentransferserie die Verbindungen $\left[\mathrm{MoO}(\mathrm{prz})_{2}\right]^{2-}$ und $\left[\mathrm{MoO}(\mathrm{mpt})_{2}\right]^{2-}$.

Tabelle 2.21 zeigt die wichtigsten Strukturparameter der Pyrazinverbindungen, Tabelle 2.22 die der Molybdopterinverbindungen. Die Nomenklatur ist analog zu den vorangegangenen Kapiteln, 7a-c bezeichnen die Verbindungen $\left[\mathrm{MoO}(\mathrm{prz})_{2}\right]^{z-}$ mit z $=-2,-1,0 ; \mathbf{8 a}-\mathbf{c}$ die Verbindungen $\left[\mathrm{WO}(\mathrm{prz})_{2}\right]^{z-}$ mit $\mathrm{z}=-2,-1,0 ;$ analog dazu 9ac die Verbindungen $\left[\mathrm{MoO}(\mathrm{mpt})_{2}\right]^{z-}$ mit $\mathrm{z}=-2,-1,0$ und 10a-c die Verbindungen $\left[\mathrm{WO}(\mathrm{mpt})_{2}\right]^{z-}$ mit $\mathrm{z}=-2,-1,0$.

Die wichtigsten das Metall betreffenden Parameter unterliegen den gleichen Trends der bereits in vorangegangenen Kapiteln besprochenen Systeme. Die Metall-Sauerstoff, die Metall-Schwefel- und die Schwefel-Kohlenstoff-Bindungen verkürzen sich beim Oxidieren von IV zu VI, während die C-C-Doppelbindung aufgeweitet wird. Die Metall-Sauerstoff-Bindungen sind bei diesen beiden Liganden bei den Wolframverbindungen signifikant länger als die der Molybdänverbindungen im Vergleich zu den bisher besprochenen Systemen. Sie liegen bei $1.7201 \AA$ (Wolframpyrazin IV als Bsp.) und sinken auf $1.7047 \AA$ in OS VI ab, während sie bei den entsprechenen Molybdänverbindungen $1.7087 \AA$ in OS IV betragen und auf $1.6962 \AA$ in OS VI absinken. Wiederum tritt die Faltung eines Liganden (S(4)-S(5)-Ebene) auf und zwar steigt der Faltungswinkel beim Wechsel in die OS V stark und beim Wechsel in die OS VI etwas schwächer an.

Die Metall-Schwefel-Bindungen sowie die C-C-Doppelbindungen sind beim Wolfram wiederum kürzer als beim Molybdän. Weiterhin ist zu beobachten, dass die vier 
Tabelle 2.21: Ausgewählte Geometrieparameter der Verbindungen $\left[\mathrm{MO}(\mathrm{prz})_{2}\right]^{z-}(\mathrm{M}$ $=$ Mo, $\mathrm{W} ; \mathrm{z}=-2,-1,0)$

\begin{tabular}{lcccccc}
\hline & $\mathbf{7 a}$ & $\mathbf{7 b}$ & $\mathbf{7 c}$ & $\mathbf{8 a}$ & $\mathbf{8 b}$ & $\mathbf{8 c}$ \\
\hline $\mathrm{M}(1)-\mathrm{S} 4$ & $0.759(5)$ & $0.799(9)$ & $0.844(7)$ & $0.724(9)$ & $0.766(5)$ & $0.788(5)$ \\
$\mathrm{M}(1)-\mathrm{O}(17)$ & 1.7108 & 1.7059 & 1.6988 & 1.7220 & 1.7138 & 1.7054 \\
$\mathrm{M}(1)-\mathrm{S}(2)$ & 2.4728 & 2.4495 & 2.4396 & 2.4524 & 2.4408 & 2.4237 \\
$\mathrm{M}(1)-\mathrm{S}(3)$ & 2.4690 & 2.4440 & 2.43191 & 2.4485 & 2.4365 & 2.4143 \\
$\mathrm{M}(1)-\mathrm{S}(4)$ & 2.4728 & 2.4350 & 2.4132 & 2.4524 & 2.4321 & 2.3968 \\
$\mathrm{M}(1)-\mathrm{S}(5)$ & 2.4690 & 2.43432 & 2.4100 & 2.4486 & 2.4316 & 2.4008 \\
$\mathrm{~S}(2)-\mathrm{C}(6)$ & 1.7782 & 1.75823 & 1.7294 & 1.7858 & 1.7671 & 1.7410 \\
$\mathrm{~S}(3)-\mathrm{C}(7)$ & 1.7840 & 1.7632 & 1.73210 & 1.7909 & 1.7722 & 1.7435 \\
$\mathrm{~S}(4)-\mathrm{C}(11)$ & 1.7782 & 1.7695 & 1.7461 & 1.7857 & 1.7746 & 1.7568 \\
$\mathrm{~S}(5)-\mathrm{C}(10)$ & 1.7840 & 1.7723 & 1.7485 & 1.7909 & 1.7775 & 1.7565 \\
$\mathrm{C}(6)-\mathrm{C}(7)$ & 1.3419 & 1.3466 & 1.3671 & 1.3394 & 1.3417 & 1.3588 \\
$\mathrm{C}(10)-\mathrm{C}(11)$ & 1.3419 & 1.3486 & 1.3693 & 1.3394 & 1.3455 & 1.3642 \\
$\mathrm{O}(17)-\mathrm{M}(1)-[\mathrm{S}(2) \mathrm{S}(3)]$ & 114.15 & 117.90 & 123.58 & 113.40 & 116.52 & 121.55 \\
$\mathrm{O}(17)-\mathrm{M}(1)-[\mathrm{S}(4) \mathrm{S}(5)]$ & 114.15 & 114.12 & 111.98 & 113.40 & 113.34 & 110.92 \\
$\mathrm{FW} \mathrm{M}(1)-\mathrm{S}(2)-\mathrm{S}(3)$ & 13.93 & 6.32 & 10.94 & 12.78 & 2.09 & 16.03 \\
$\mathrm{FW} \mathrm{M}(1)-\mathrm{S}(4)-\mathrm{S}(5)$ & 13.93 & 32.52 & 35.39 & 12.79 & 31.96 & 40.48 \\
$\mathrm{C}(13)-\mathrm{O}(8)$ & 1.4361 & 1.4238 & 1.4165 & 1.4368 & 1.4239 & 1.4145 \\
$\mathrm{O}(8)-\mathrm{C}(15)$ & 1.4236 & 1.4289 & 1.4291 & 1.4239 & 1.4291 & 1.4328 \\
$\mathrm{C}(19)-\mathrm{O} 21)$ & 1.4361 & 1.4241 & 1.4153 & 1.4369 & 1.4239 & 1.4135 \\
$\mathrm{O}(21)-\mathrm{C}(22)$ & 1.4236 & 1.4286 & 1.4319 & 1.4240 & 1.4291 & 1.4324 \\
$\mathrm{C}(7)-\mathrm{C}(6)-\mathrm{C}(13)-\mathrm{O}(8)$ & -10.85 & -12.95 & -16.20 & -10.68 & -12.25 & -14.83 \\
$\mathrm{C}(7)-\mathrm{C}(14)-\mathrm{C}(15)-\mathrm{O}(8)$ & 50.99 & 51.74 & 51.28 & 50.96 & 51.78 & 51.92 \\
$\mathrm{C}(10)-\mathrm{C}(11)-\mathrm{C}(19)-\mathrm{O}(21)$ & -10.85 & -10.11 & -14.60 & -10.68 & -9.81 & -12.00 \\
$\mathrm{C}(10)-\mathrm{C}(20)-\mathrm{C}(22)-\mathrm{O}(21)$ & 50.99 & 52.19 & 51.02 & 50.96 & 52.30 & 51.72 \\
& & & & & &
\end{tabular}

Metall-Schwefelbindungen unterschiedliche Längen aufweisen. In Oxidationsstufe IV sind die Abstände $\mathrm{M}(1)-\mathrm{S}(2)$ (2.4710 $\AA$, Mo; $2.4506 \AA$, W) und M(1)-S(4) (2.4710 $\AA, \mathrm{Mo} ; 2.4506 \AA, \mathrm{W})$ sowie $\mathrm{M}(1)-\mathrm{S}(3)(2.4680 \AA, \mathrm{Mo} ; 2.4359 \AA, \mathrm{W})$ und $\mathrm{M}(1)-\mathrm{S}(5)$ $(2.4680 \AA$, Mo; $2.4314 \AA$, W) gleich lang. Dabei sind die Bindungen des ersten Paares etwas länger. In OS V dagegen erfolgt eine Abnahme der Bindungslängen von M(1)$\mathrm{S}(2)$ bis zu M(1)-S(5), wobei sich die Bindungslängen von $M(1)-S(2)$ zu M(1)-S(3) und $\mathrm{M}(1)-\mathrm{S}(4)$ zu M(1)-S(5) nur sehr geringfügig ändern, also insgesamt die beiden zu einem Liganden gehörigen Schwefel-Atome ähnliche Abstände zum Metall aufweisen. 
Tabelle 2.22: Ausgewählte Geometrieparameter der Verbindungen $\left[\mathrm{MO}(\mathrm{mpt})_{2}\right]^{z-}(\mathrm{M}$ $=$ Mo, $\mathrm{W} ; \mathrm{z}=-2,-1,0)$

\begin{tabular}{lcccccc}
\hline & $\mathbf{9 a}$ & $\mathbf{9 b}$ & $\mathbf{9 c}$ & $\mathbf{1 0 a}$ & $\mathbf{1 0 b}$ & $\mathbf{1 0 c}$ \\
\hline $\mathrm{M}(1)-\mathrm{S} 4$ & $0.761(2)$ & $0.805(2)$ & $0.847(2)$ & $0.737(0)$ & $0.755(4)$ & $0.788(3)$ \\
$\mathrm{M}(1)-\mathrm{O}(19)$ & 1.7087 & 1.7038 & 1.6962 & 1.7201 & 1.7119 & 1.7047 \\
$\mathrm{M}(1)-\mathrm{S}(2)$ & 2.4710 & 2.4504 & 2.4378 & 2.4506 & 2.4412 & 2.4211 \\
$\mathrm{M}(1)-\mathrm{S}(3)$ & 2.4680 & 2.4439 & 2.4314 & 2.4474 & 2.4359 & 2.4153 \\
$\mathrm{M}(1)-\mathrm{S}(4)$ & 2.4710 & 2.4358 & 2.4139 & 2.4506 & 2.43310 & 2.3989 \\
$\mathrm{M}(1)-\mathrm{S}(5)$ & 2.4680 & 2.4345 & 2.4170 & 2.4474 & 2.4314 & 2.3987 \\
$\mathrm{~S}(2)-\mathrm{C}(6)$ & 1.7758 & 1.7572 & 1.7298 & 1.7834 & 1.7658 & 1.7413 \\
$\mathrm{~S}(3)-\mathrm{C}(7)$ & 1.7820 & 1.7627 & 1.7312 & 1.7893 & 1.7718 & 1.7433 \\
$\mathrm{~S}(4)-\mathrm{C}(11)$ & 1.7758 & 1.7691 & 1.7466 & 1.7834 & 1.7737 & 1.7567 \\
$\mathrm{~S}(5)-\mathrm{C}(10)$ & 1.7820 & 1.7719 & 1.7491 & 1.7893 & 1.7765 & 1.7580 \\
$\mathrm{C}(6)-\mathrm{C}(7)$ & 1.3435 & 1.3476 & 1.3672 & 1.3410 & 1.3426 & 1.3589 \\
$\mathrm{C}(10)-\mathrm{C}(11)$ & 1.3435 & 1.3481 & 1.3671 & 1.3410 & 1.3457 & 1.3639 \\
$\mathrm{O}(19)-\mathrm{M}(1)-[\mathrm{S}(2) \mathrm{S}(3)]$ & 114.34 & 117.89 & 123.44 & 113.56 & 116.44 & 121.87 \\
$\mathrm{O}(19)-\mathrm{M}(1)-[\mathrm{S}(4) \mathrm{S}(5)]$ & 114.34 & 114.17 & 112.26 & 113.56 & 113.34 & 110.29 \\
$\mathrm{FW} \mathrm{S}(2)-\mathrm{S}(3)$ & 12.56 & 8.23 & 12.39 & 11.51 & 3.45 & 17.69 \\
$\mathrm{FW} \mathrm{S}(4)-\mathrm{S}(5)$ & 12.56 & 31.23 & 34.50 & 11.51 & 30.87 & 40.04 \\
$\mathrm{C}(16)-\mathrm{O}(8)$ & 1.4393 & 1.4256 & 1.4171 & 1.4401 & 1.4259 & 1.4153 \\
$\mathrm{O}(8)-\mathrm{C}(18)$ & 1.4161 & 1.4216 & 1.4251 & 1.4165 & 1.4217 & 1.4279 \\
$\mathrm{C}(25)-\mathrm{O}(27)$ & 1.4393 & 1.4262 & 1.4178 & 1.4401 & 1.4260 & 1.4154 \\
$\mathrm{O}(27)-\mathrm{C}(28)$ & 1.4161 & 1.4212 & 1.4245 & 1.4165 & 1.4215 & 1.4290 \\
$\mathrm{C}(7)-\mathrm{C}(6)-\mathrm{C}(16)-\mathrm{O}(8)$ & -8.06 & -12.29 & -15.15 & -7.79 & -11.61 & -15.18 \\
$\mathrm{C}(7)-\mathrm{C}(17)-\mathrm{C}(18)-\mathrm{O}(8)$ & 51.56 & 50.80 & 51.14 & 51.55 & 50.72 & 50.32 \\
$\mathrm{C}(10)-\mathrm{C}(11)-\mathrm{C}(25)-\mathrm{O}(27)$ & -8.06 & -9.16 & -13.67 & -7.79 & -8.83 & -12.57 \\
$\mathrm{C}(10)-\mathrm{C}(26)-\mathrm{C}(28)-\mathrm{O}(27)$ & 51.56 & 51.14 & 48.90 & 51.51 & 51.18 & 49.84 \\
& & & & & &
\end{tabular}

In OS VI ist dieser Trend noch weiter verstärkt, dabei sind die zum gefalteten Liganden gehörenden Bindungslängen M(1)-S(4) und M(1)-S(5) (2.4139/2.4170 $\AA$ Mo, 2.3989/2.3989 § W) gegeüber den Bindungslängen M(1)-S(2) und M(1)-S(3) mit 2.4378/2.4314 $\AA$ für Mo und 2.4211/2.4153 $\AA$ für W um etwa 0.02 A verkürzt. Dieser bereits bei den Chromanyl- und Flavanylverbindungen beobachtete Trend ist bei den Pyrazin- und Molybdopterin-Systemen weniger ausgeprägt. So betragen bspw. die Unterschiede der Flavanylkomplexe des Molybdän in OS IV zwischen M(1)-S(2) und M(1)-S(3) $0.015 \AA$ die bei den entsprechenden Pyrazinkomplexen nur $0.004 \AA$ und bei den Molybdopterinkomplexen nur noch $0.003 \AA$. 
Analog dazu verhalten sich die angrenzenden Schwefel-Kohlenstoff-Bindungen; die an die längere $\mathrm{M}(1)-\mathrm{S}(2)$-Bindung geknüpfte $\mathrm{S}(2)-\mathrm{C}(6)$-Bindung ist kürzer als die benachbarte $\mathrm{S}(3)-\mathrm{C}(6)$-Bindung welche an die kürzere $\mathrm{M}(1)-\mathrm{S}(3)$-Bindung grenzt. Dies gilt für alle S-C-Bindungen, wobei deren Längenunterschiede größer sind als die der M-S-Bindungen.

Im Vergleich zu den anderen Systemen sind die Bindungen bei den beiden Modellen, sowohl den Pyrazin- als auch den Molybdopterinsystemen, marginal länger, vor allem in Bezug auf die Pyranylsysteme, wobei die Geometrien der aromatischen Systeme den berechneten kompletten Kofaktoren recht nahe kommen. Am deutlichsten ist das an der Anhebung des Metalls aus der $\mathrm{S}_{4}$-Ebene zu sehen, die sich zwischen $0.76 \AA(\mathbf{7 a}$ und $\mathbf{9 a}$ in OS IV ) und $0.84 \AA$ (7c und 9c in OS VI) bewegt. Diese Werte wurden auch für die aromatischen Modellverbindungen erhalten. Wiederum erfolgt ein gleichmäßiger Anstieg von jeweils $0.04 \AA$ (W $0.02 \AA$ ) pro Oxidationsschritt. Die Wolframverbindungen verbleiben flacher und zeigen eine geringere Änderung mit zunehmender OS.

Der auffälligste Unterschied ist an den im Pyranring liegenden C-O-Bindungen zu beobachten. Während bei den aromatischen Systemen ein großer Unterschied zwischen den beiden den Pyransauerstoff einschließenden Bindungen auftritt 14 sind die Unterschiede bei den Pyrazin- und Molybdopterinliganden wesentlich geringer. Wiederum ist die C-O-Bindung, welche näher an der C-C-Doppelbindung liegt, länger als die an den Pyrazinring grenzende. So beträgt die Bindungslänge C(16)-O(8) 1.4393 $\AA$ (9a in OS IV) gegenüber C(8)-O(16) mit nur 1.4161 $\AA$. Insgesamt ist eine leichte Verkürzung mit zunehmender OS zu verzeichnen, so verringern sich die Abstände in OS VI für C(16)-O(8) auf $1.4171 \AA$ (9c) und für $\mathrm{C}(8)-\mathrm{O}(16)$ auf $1.4152 \AA$.

Die Pyransauerstoffe sind wieder aus dem Ring heraus in Richtung Metall in der

\footnotetext{
${ }^{14}$ Mo-Chromanyl in OS IV: C(8)-O(9) $1.445 \AA, \mathrm{C}(9)-\mathrm{O}(16) 1.365 \AA$
} 
endo-Form angehoben. Im Gegensatz zu den aromatischen Systemen ist diese Konformation bei den Liganden auf beiden Seiten und in allen OS gleich. Scheinbar bieten die aromatischen Nachbarn stereochemisch mehr Freiheit, während die angrenzenden Stickstoffatome im Nachbarring die Beweglichkeit des Sauerstoffs stark einschränken und ihn, um die Abstoßung zu minimieren, in die endo-Form zwingen.

Da die Konformation durch den angrenzenden Ring stark beeinflusst ist, liegen die den Pyranring betreffenden Diederwinkel in anderen Größenordnungen als die der aromatischen Systemen und sind in dem Punkt auch nicht mehr vergleichbar.

Bezogen auf die von der C-C-Doppelbindung und den folgenden in Richtung Pyransauerstoff liegenden C-Atomen aufgespannten Ebene erhöht sich der Diederwinkel $\mathrm{C}(7)-\mathrm{C}(6)-\mathrm{C}(16)-\mathrm{O}(8)$ nur leicht von $10.85^{\circ}$ bis $16.20^{\circ}$ (7, OS IV bis OS VI) und von $8.06^{\circ}$ auf $15.15^{\circ}(\mathbf{9}$, OS IV bis OS VI). Die Werte für die Wolframverbindungen liegen etwas darunter, auch ist die Änderung nicht so stark. Dieses Abknicken ist in den Molybdopterinverbindungen in OS IV etwas schwächer, steigt aber auf ähnliche Werte wie die der Pyrazinverbindungen an. Unterschiede tauchen zwischen den beiden Liganden auf. In dem gefalteten Liganden verbleibt der o.g. Winkel erst bei $10.85^{\circ} / 8.06^{\circ}$ (Molybdänpyrazin, Molybdänmolybdopterin) und steigt dann beim Wechsel in die OS VI auf $14.60^{\circ}$ (Molybdänpyrazin) und $13.67^{\circ}$ (Molybdänmolybdopterin) an. Der von der gegenüberliegenden Seite des Pyranringes ausgehende Diederwinkel beträgt ca. $50^{\circ}$ und ändert sich im Laufe der Oxidation nur recht wenig $\left(+-1^{\circ}\right)$.

\subsubsection{Elektronische Struktur und Bindung}

Die NPA-Ladungen beider Systeme sind in Abbildung 2.19] als Diagramme dargestellt. Dabei sind einmal die das Metallzentrum betreffenden Atome für beide Systeme dargestellt sowie die Ladungen an den Verbindungen $\left[\mathrm{MO}(\mathrm{mpt})_{2}\right]^{2-}(\mathrm{M}=\mathrm{Mo}$, W) (Abbildung 2.20). Die Absolutwerte sowie ihre Änderungstendenzen mit zuneh- 
Abbildung 2.19: NPA-Ladungen der Metallzentren $\left[\mathrm{MO}(\mathrm{prz})_{2}\right]^{z-}$ und $\left.\mathrm{MO}(\mathrm{mpt})_{2}\right]^{z-}(\mathrm{M}$ $=$ Mo, $\mathrm{W} ; \mathrm{z}=-2,-1,0)$
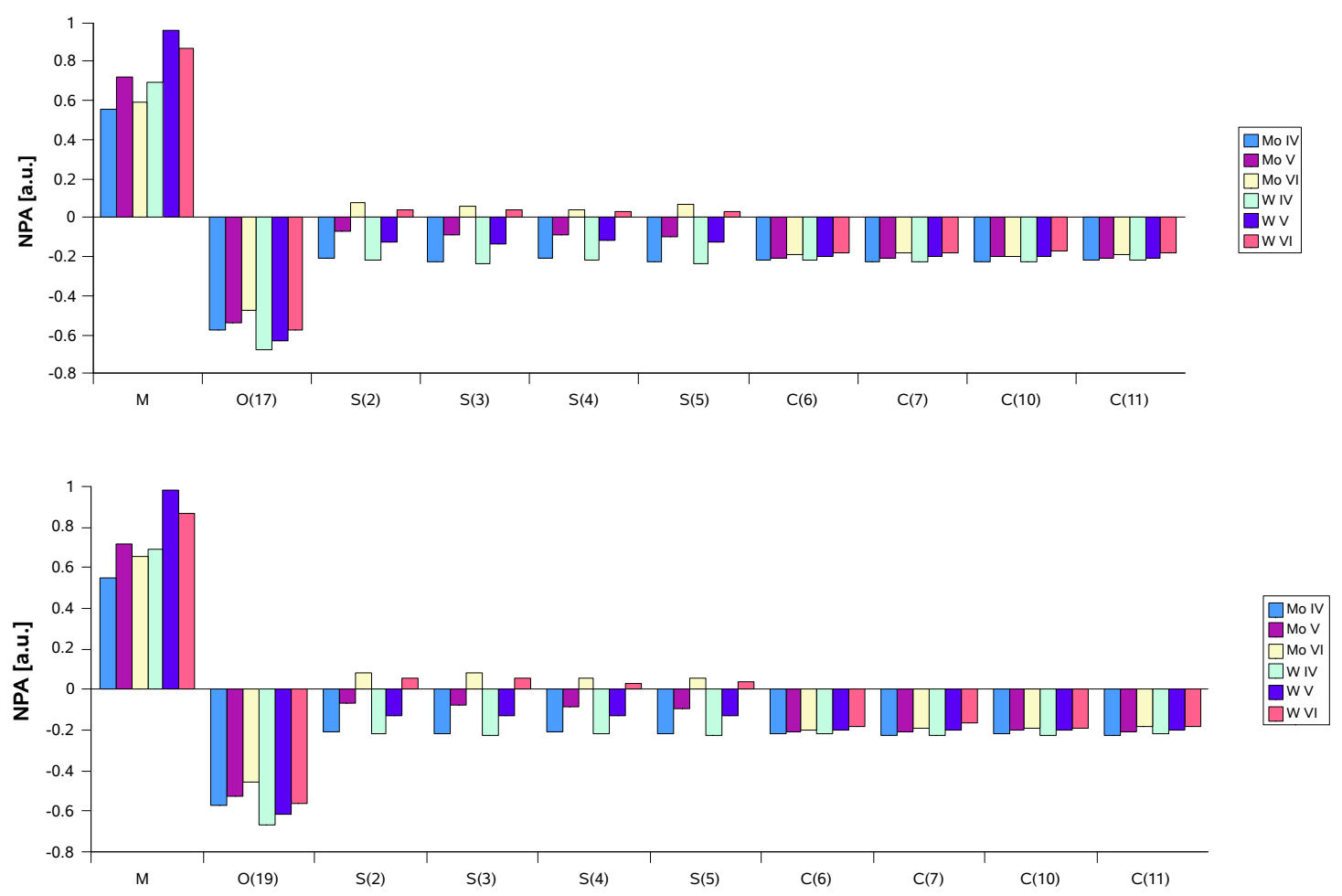

mender Oxidation entsprechen denen der Chromanyl- und Flavanylsysteme. D.h. ein großer Einfluss des Pteridingerüsts ist nicht erkennbar. So sind auch keine großen Unterschiede beim Wechsel vom Modell Pyrazin zum Molybdopterin zu beobachten.

Generelle Trends treten auch hier wieder auf, so das Ansteigen der Ladung am Metall beim Wechsel von OS IV zu OS V, die beim Wolfram höher ausfällt, sowie der Rückgang in OS VI. Demgegenüber sind die Ladungen an den SchwefelAtomen bei den Wolfram-Verbindungen insgesamt niedriger. Die Ladungen an der C-C-Doppelbindung dagegen weisen keinen großen Unterschied mehr auf. Genauso verhält es sich auch beim Wechsel der OS; je weiter weg sich ein Atom vom Metallzentrum befindet, desto geringer wird die Änderung der Ladung.

Die Bindungsanteile sind in den OS IV und V analog zu den bisher besprochenen 
Abbildung 2.20: NPA-Ladungen für $\left.\mathrm{MO}(\mathrm{mpt})_{2}\right]^{z-}(\mathrm{M}=\mathrm{Mo}, \mathrm{W} ; \mathrm{z}=-2)$

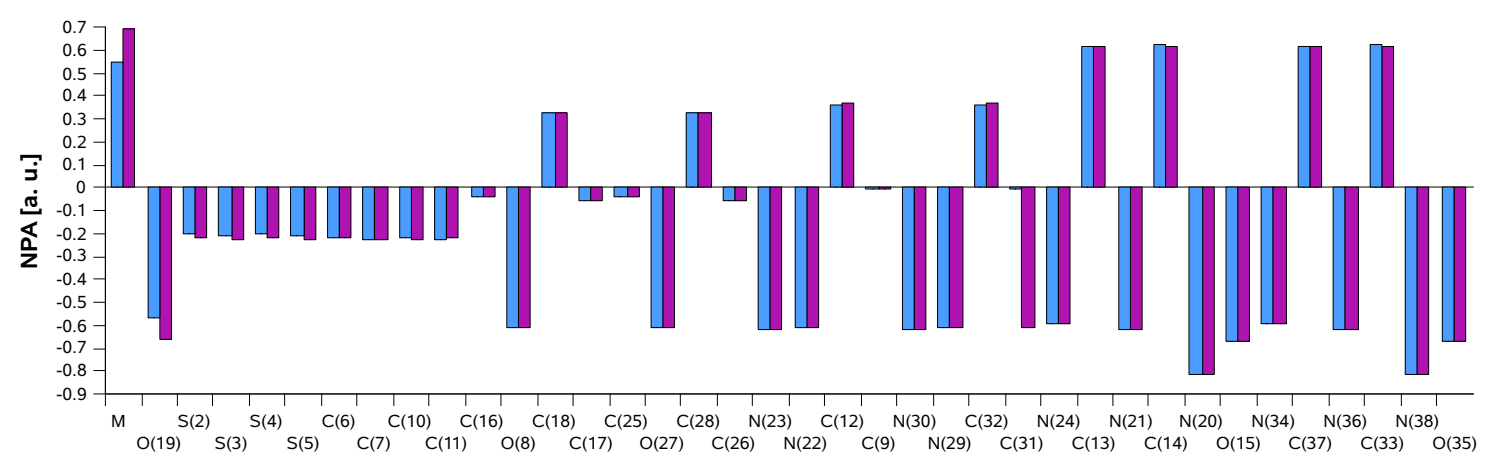

Strukturen. Im Gegensatz zu den aromatischen Systemen ist eine leichte konjugierende Wechselwirkung der freien Elektronenpaare der Stickstoffatome zu beobachten. In den Tabellen 2.24 und 2.25 sind derartige Interaktionen am Beispiel der Pyrazinringe und des Pyrimidinrings dargestellt (in OS IV). So finden sich Anteile der C-CDoppelbindung des Pyrazinringes $(\mathrm{C}(9), \mathrm{C}(12))$ an den freien Elektronenpaaren der Stickstoffatome N(16) und N (18) der Pyrazinverbindung (Mo, OS IV), welche in einer Größenordnung zwischen 2 und 5\% liegen. Bei der entsprechenden Molybdopterinverbindung (Mo, OS IV) sind diese Wechselwirkungen von ähnlicher Größe und betragen $5 \%$ für den Anteil von $\mathrm{C}(9)$ am freien Elektronenpaar von $\mathrm{N}(23)$ und 5 bzw. $3 \%$ für die Anteile von C(9) bzw. C(12) am freien Elektronenpaar vom Atom N(22). Zwischen den Pyrazin- und Molybdopterinsystemen sowie innerhalb der verschiedenen OS besteht dabei kein großer Unterschied. Der im Molybdopterinsystem hinzukommende dritte Ring weist wiederum zahlreiche Beimischungen der freien Elektronenpaare des Stickstoffs mit seinen jeweiligen Nachbaratomen auf. Recht viele Anteile hat dabei das Atom N(21) mit Beimischungen von $\mathrm{C}(13)$ mit fast 8\%, C(14) mit 4\%, N(24) mit $3 \%$ sowie geringeren Anteilen von $\mathrm{C}(12)$ und $\mathrm{O}(15)$.

Die Metallzentren beider Systeme in OS VI weisen wiederum die Besonderheit der Ausbildung von Metall-Schwefel-Doppelbindungen auf und zwar gleichmermaßen bei beiden Metallen. Bei der Pyrazinverbindung handelt es sich bei der Molybdänverbin- 
Tabelle 2.23: NBO-Parameter der Bindungen $\mathrm{M}^{V I}$-S-Doppelbindungen

\begin{tabular}{|c|c|c|c|c|c|c|c|c|c|c|}
\hline \multirow[t]{2}{*}{ A-B } & \multirow{2}{*}{\multicolumn{2}{|c|}{$\% \mathrm{~A}$}} & \multicolumn{3}{|c|}{ Hybridisierung } & \multirow[t]{2}{*}{$\mathrm{B}^{a}$} & \multirow[t]{2}{*}{$\% \mathrm{~B}$} & \multicolumn{3}{|c|}{ Hybridisierung } \\
\hline & & & $\mathrm{s}$ & $\mathrm{p}$ & $\mathrm{d}$ & & & $\mathrm{s}$ & $\mathrm{p}$ & $\mathrm{d}$ \\
\hline \multicolumn{11}{|c|}{$\left[\mathrm{Mo}^{V I} \mathrm{O}(\mathrm{prz})_{2}\right]$} \\
\hline Mo-S(2) & $\sigma$ & 23.77 & 11.60 & 0.74 & 87.66 & & 72.99 & 16.35 & 83.51 & 0.14 \\
\hline \multirow[t]{4}{*}{ Mo-S(2) } & $\pi$ & 41.99 & 0.06 & 0.10 & 99.84 & & 52.04 & 0.05 & 99.84 & 0.10 \\
\hline & & & & & & $\mathrm{C}(6)$ & $<2$ & & & \\
\hline & & & & & & $\mathrm{C}(7)$ & $<2$ & & & \\
\hline & & {$\left[\mathrm{W}^{V I} \mathrm{C}\right.$} & $\left.(\mathrm{prz})_{2}\right]$ & & & & & & & \\
\hline $\mathrm{W}-\mathrm{S}(5)$ & $\sigma$ & 25.58 & 16.87 & 1.17 & 81.96 & & 71.89 & 17.44 & 82.35 & 0.20 \\
\hline \multirow[t]{4}{*}{ W-S(5) } & $\pi$ & 32.46 & 0.16 & 0.20 & 99.64 & & 61.86 & 0.28 & 99.63 & 0.09 \\
\hline & & & & & & $\mathrm{C}(10)$ & $<2$ & & & \\
\hline & & & & & & $\mathrm{C}(11)$ & $<2$ & & & \\
\hline & & {$\left[\mathrm{Mo}^{V I} \mathrm{C}\right.$} & $(\mathrm{mpt})_{2}$ & & & & & & & \\
\hline Mo-S(2) & $\sigma$ & 23.92 & 12.41 & 0.69 & 86.90 & & 73.22 & 16.57 & 83.29 & 0.14 \\
\hline \multirow[t]{4}{*}{ Mo-S(2) } & $\pi$ & 39.79 & 0.05 & 0.10 & 99.85 & & 54.10 & 0.07 & 99.83 & 0.10 \\
\hline & & & & & & $\mathrm{C}(6)$ & $<2$ & & & \\
\hline & & & & & & $\mathrm{C}(7)$ & $<2$ & & & \\
\hline & & {$\left[\mathrm{W}^{V I} \mathrm{O}\right.$} & $\left.(\mathrm{mpt})_{2}\right]$ & & & & & & & \\
\hline $\mathrm{W}-\mathrm{S}(2)$ & $\sigma$ & 22.37 & 17.32 & 1.13 & 81.55 & & 74.16 & 18.68 & 81.15 & 0.17 \\
\hline \multirow[t]{3}{*}{$\mathrm{W}-\mathrm{S}(2)$} & $\pi$ & 26.07 & 0.22 & 0.30 & 99.47 & & 66.89 & 0.16 & 99.75 & 0.09 \\
\hline & & & & & & $\mathrm{C}(10)$ & $<2$ & & & \\
\hline & & & & & & $\mathrm{C}(11)$ & $<2$ & & & \\
\hline
\end{tabular}

${ }^{a}$ Die Spalte "B" beinhaltet wechselwirkende Nachbaratome, die nicht direkt zur Bindung gehören.

dung um die Bindung Mo-S(3), bei der Wolframverbindung um die Bindung W-S(2) und beim Molybdopterinsystem bei beiden Metallen um die Bindung M-S(2). Wiederum sind auch Beimischungen der verbleibenden freien Elektronenpaare der Nachbarschwefelatome sowie der C-C-Doppelbindung zum Metall vorhanden. Veränderungen innerhalb der verschiedenen OS und sind am Beispiel des Molybdänpyrazinkomplexes anhand der freien Elektronenpaare von $\mathrm{S}(2)$ in Tabelle 2.23 gezeigt. Die Anteile des Metalls an den freien Elektronenpaaren des Schwefels steigen von 3.9/4.1\% in den OS IV $/ \mathrm{V}$ auf bis zu $15 \%$ in OS IV. Damit ist eine Tendenz zur Bildung einer weiteren 
M-S-Doppelbindung angedeutet.

Tabelle 2.24: NBO-Parameter ausgewählter freier Elektronenpaare von $\left[\mathrm{MoO}(\mathrm{prz})_{2}\right]^{z-}$

\begin{tabular}{|c|c|c|c|c|c|c|c|c|c|}
\hline \multirow[t]{2}{*}{ A-B } & \multirow[t]{2}{*}{$\% \mathrm{~A}$} & \multicolumn{3}{|c|}{ Hybridisierung } & \multirow[t]{2}{*}{$\mathrm{B}^{a}$} & \multirow[t]{2}{*}{$\% \mathrm{~B}$} & \multicolumn{3}{|c|}{ Hybridisierung } \\
\hline & & $\mathrm{s}$ & $\mathrm{p}$ & $\mathrm{d}$ & & & $\mathrm{s}$ & $\mathrm{p}$ & $\mathrm{d}$ \\
\hline \multicolumn{10}{|c|}{$\left[\mathrm{Mo}^{I V} \mathrm{O}(\mathrm{prz})_{2}\right]^{2-}$} \\
\hline LP Mo(1) & 95.58 & 0.04 & 0.04 & 99.92 & & & & & \\
\hline LP S(2) & 98.75 & 62.89 & 37.10 & 0.02 & & & & & \\
\hline \multirow[t]{4}{*}{ LP S(2) } & 86.90 & 0.00 & 99.96 & 0.04 & $\operatorname{Mo}(1)$ & 3.90 & 0.71 & 0.96 & 98.34 \\
\hline & & & & & $\mathrm{C}(6)$ & 3.84 & 0.01 & 98.78 & 1.22 \\
\hline & & & & & $\mathrm{C}(7)$ & 3.10 & 0.31 & 99.65 & 0.04 \\
\hline & \multicolumn{4}{|c|}{$\left[\mathrm{Mo}^{V} \mathrm{O}(\mathrm{prz})_{2}\right]^{1-}$} & & & & & \\
\hline LP $\mathrm{Mo}(1)$ & 97.36 & 0.02 & 0.02 & 99.96 & & & & & \\
\hline LP S(2) & 98.77 & 63.08 & 36.90 & 0.02 & & & & & \\
\hline \multirow[t]{4}{*}{ LP S(2) } & 87.19 & 1.52 & 98.45 & 0.04 & $\operatorname{Mo}(1)$ & 4.08 & 3.88 & 0.78 & 95.34 \\
\hline & & & & & $\mathrm{C}(6)$ & 3.63 & 0.01 & 98.69 & 1.31 \\
\hline & & & & & $\mathrm{C}(7)$ & 3.04 & 0.01 & 99.97 & 0.02 \\
\hline & \multicolumn{4}{|c|}{$\left[\mathrm{Mo}^{V I} \mathrm{O}(\mathrm{prz})_{2}\right]$} & & & & & \\
\hline \multicolumn{10}{|l|}{ LP Mo(1) } \\
\hline LP S(2) & 98.27 & 63.94 & 36.04 & 0.02 & & & & & \\
\hline LP S(3) & 98.21 & 63.38 & 36.60 & 0.02 & & & & & \\
\hline \multirow[t]{4}{*}{ LP S(3) } & 63.07 & 0.10 & 99.83 & 0.07 & $\operatorname{Mo}(1)$ & 14.79 & 0.05 & 0.13 & 99.82 \\
\hline & & & & & $\mathrm{S}(2)$ & 10.40 & 0.02 & 99.57 & 0.41 \\
\hline & & & & & $\mathrm{C}(6)$ & 5.09 & 0.67 & 99.21 & 0.12 \\
\hline & & & & & $\mathrm{C}(7)$ & 5.09 & 0.37 & 98.96 & 0.67 \\
\hline
\end{tabular}

${ }^{a}$ Die Spalte "B" beinhaltet wechselwirkende Nachbaratome, die nicht direkt zur Bindung gehören.

Die Metall-Schwefel-Doppelbindungen, stammend aus dem freien Elektronenpaar des Schwefels, enthalten bei den Pyrazinverbindungen 42\% Metall- und 52\% Schwefelanteil bei der Molybdänverbindung und 32\% Metall- und $62 \%$ Schwefelanteil bei der Wolframverbindung. 
Tabelle 2.25: Ausgewählte NBO-Parameter der N-haltigen Ringe

\begin{tabular}{|c|c|c|c|c|c|c|c|c|c|}
\hline \multirow{2}{*}{$\begin{array}{l}\text { A-B } \\
7 \mathbf{a}\end{array}$} & \multirow[t]{2}{*}{$\% \mathrm{~A}$} & \multicolumn{3}{|c|}{ Hybridisierung } & \multirow[t]{2}{*}{$\mathrm{B}^{a}$} & \multirow[t]{2}{*}{$\% \mathrm{~B}$} & \multicolumn{3}{|c|}{ Hybridisierung } \\
\hline & & $\mathrm{s}$ & $\mathrm{p}$ & d & & & s & $\mathrm{p}$ & $\mathrm{d}$ \\
\hline \multirow{2}{*}{ LP N(18) } & 92.54 & 12.29 & 87.68 & 0.03 & $\mathrm{C}(9)$ & 2.16 & 0.85 & 99.00 & 0.16 \\
\hline & & & & & $\mathrm{C}(12)$ & 2.05 & 0.19 & 96.84 & 2.97 \\
\hline \multirow[t]{2}{*}{ LP N(16) } & 89.50 & 7.78 & 92.19 & 0.03 & $\mathrm{C}(9)$ & 4.62 & 0.19 & 98.05 & 1.76 \\
\hline & & & & & $\mathrm{C}(12)$ & 4.04 & 0.06 & 99.89 & 0.05 \\
\hline \multicolumn{10}{|l|}{$9 c$} \\
\hline LP N(23) & 87.41 & 4.99 & 94.99 & 0.02 & $\mathrm{C}(12)$ & 5.33 & 0.03 & 98.36 & 1.60 \\
\hline \multirow[t]{2}{*}{ LP N(22) } & 88.00 & 6.59 & 93.38 & 0.03 & $\mathrm{C}(9)$ & 5.20 & 0.12 & 98.11 & 1.76 \\
\hline & & & & & $\mathrm{C}(12)$ & 3.19 & 0.07 & 99.77 & 0.15 \\
\hline $9 a$ & $\% \mathrm{~A}$ & \multicolumn{3}{|c|}{ Hybridisierung } & B & $\% \mathrm{~B}$ & \multicolumn{3}{|c|}{ Hybridisierung } \\
\hline A-B & & $\mathrm{s}$ & $\mathrm{p}$ & $\mathrm{d}$ & & & s & $\mathrm{p}$ & $\mathrm{d}$ \\
\hline \multirow[t]{6}{*}{$\mathrm{C}(9)-\mathrm{C}(12)$} & 49.81 & 30.68 & 69.28 & 0.04 & & 49.09 & 34.81 & 65.15 & 0.04 \\
\hline & 47.04 & 0.02 & 99.90 & 0.08 & & 36.72 & 0.02 & 99.90 & 0.08 \\
\hline & & & & & $\mathrm{C}(14)$ & 8.79 & 0.01 & 99.22 & 0.77 \\
\hline & & & & & $\mathrm{O}(15)$ & 3.54 & 0.00 & 99.96 & 0.04 \\
\hline & & & & & $\mathrm{C}(13)$ & $<2$ & & & \\
\hline & & & & & $\mathrm{N}(24)$ & $<2$ & & & \\
\hline $\mathrm{C}(12)-\mathrm{N}(24)$ & 40.16 & 27.22 & 72.69 & 0.09 & & 58.70 & 34.19 & 65.73 & 0.08 \\
\hline \multirow{4}{*}{$\mathrm{C}(13)-\mathrm{N}(24)$} & 40.93 & 32.76 & 67.18 & 0.06 & & 58.30 & 36.45 & 63.45 & 0.10 \\
\hline & 33.17 & 0.00 & 99.77 & 0.22 & & 60.24 & 0.00 & 99.86 & 0.13 \\
\hline & & & & & $\mathrm{C}(12)$ & 2.77 & 0.01 & 97.97 & 2.02 \\
\hline & & & & & $\mathrm{C}(14)$ & $<2$ & & & \\
\hline $\mathrm{C}(13)-\mathrm{N}(21)$ & 37.83 & 29.61 & 70.29 & 0.09 & & 61.67 & 36.03 & 63.92 & 0.05 \\
\hline $\mathrm{C}(14)-\mathrm{N}(21)$ & 35.18 & 25.87 & 74.01 & 0.12 & & 64.12 & 34.49 & 65.47 & 0.04 \\
\hline $\mathrm{C}(9)-\mathrm{C}(14)$ & 50.80 & 28.78 & 71.17 & 0.05 & & 47.92 & 35.86 & 64.10 & 0.04 \\
\hline $\mathrm{C}(13)-\mathrm{N}(20)$ & 39.53 & 27.75 & 72.16 & 0.09 & & 59.87 & 34.89 & 65.03 & 0.08 \\
\hline \multirow[t]{2}{*}{$\mathrm{C}(14)-\mathrm{O}(15)$} & 35.51 & 29.95 & 69.91 & 0.13 & & 64.16 & 40.43 & 59.45 & 0.11 \\
\hline & 28.41 & 0.04 & 99.49 & 0.47 & & 70.95 & 0.05 & 99.85 & 0.10 \\
\hline \multirow[t]{2}{*}{ LP N(24) } & 94.62 & 27.66 & 72.27 & 0.07 & $\mathrm{C}(12)$ & $<2$ & & & \\
\hline & & & & & $\mathrm{C}(13)$ & $<2$ & & & \\
\hline \multirow[t]{2}{*}{ LP N(20) } & 93.96 & 16.68 & 83.27 & 0.05 & $\mathrm{C}(13)$ & 3.29 & 0.16 & 97.53 & 2.31 \\
\hline & & & & & $\mathrm{N}(24)$ & $<2$ & & & \\
\hline \multirow[t]{5}{*}{ LP N(21) } & 80.69 & 0.10 & 99.89 & 0.01 & $\mathrm{C}(13)$ & 7.92 & 0.02 & 98.94 & 1.04 \\
\hline & & & & & $\mathrm{C}(14)$ & 4.41 & 0.01 & 98.68 & 1.31 \\
\hline & & & & & $\mathrm{N}(24)$ & 3.42 & 0.01 & 99.93 & 0.06 \\
\hline & & & & & $\mathrm{C}(12)$ & $<2$ & & & \\
\hline & & & & & $\mathrm{O}(15)$ & $<2$ & & & \\
\hline \multirow[t]{4}{*}{ LP O(15) } & 98.90 & 59.51 & 40.47 & 0.02 & & & & & \\
\hline & 92.70 & 0.00 & 99.93 & 0.07 & $\mathrm{C}(14)$ & 4.02 & 1.18 & 95.02 & 3.80 \\
\hline & & & & & $\mathrm{C}(9)$ & $<2$ & & & \\
\hline & & & & & $\mathrm{C}(21)$ & $<2$ & & & \\
\hline
\end{tabular}

${ }^{a}$ Die Spalte "B" beinhaltet wechselwirkende Nachbaratome, die nicht direkt zur Bindung gehören. 
Beim Molybdopterinsystem sind die Anteile des Mo mit einer Zusammensetzung von $40 \%$ Metall- und 54\% Schwefelanteil höher und beim Wolfram wiederum niedriger mit 26\% Metall- und 67\% Schwefelanteil. Beide beinhalten jedoch noch Anteile der C-C-Doppelbindung in Höhe von etwa $2 \%$ pro C-Atom. Beim Molybän handelt es sich um die direkt benachbarte Bindung $\mathrm{C}(6)-\mathrm{C}(7)$, beim Wolfram hingegen um die gegenüberliegende Bindung C(10)-C(11). Auffallend am Wolframmolybdopterinkomplex in OS VI ist auch das Fehlen eines weiteren Elektronenpaars an S(5), welches in die umliegenden Bindungen umverteilt worden ist.

Die Orbitalenergien beider Systeme sind in Tabelle 2.26] dargestellt. Die Größe der HOMO-LUMO-Lücke der Pyrazinkomplexe ähnelt den Werten der Pyranylkomplexe mit Beträgen von 0.14 (W 0.13) in OS IV und 0.13 (W 0.14) OS V bis zu einem Absinken in OS VI auf 0.08 a.u.

Tabelle 2.26: Orbitalenergien von $\left[\mathrm{MO}(\mathrm{prz})_{2}\right]^{z-}$ und $\left[\mathrm{MO}(\mathrm{mpt})_{2}\right]^{z-}(\mathrm{M}=\mathrm{Mo}, \mathrm{W} ; \mathrm{z}=$ $-2,-1,0)$

\begin{tabular}{cccccccc}
\hline & Orbital & $\mathbf{7 a}$ & $\mathbf{7 b}$ & $\mathbf{7 c}$ & $\mathbf{8 a}$ & $\mathbf{8 b}$ & $\mathbf{8 c}$ \\
\hline & 114 & 0.03 & -0.09 & -0.19 & 0.03 & -0.09 & -0.18 \\
& 115 & 0.05 & -0.09 & -0.18 & 0.05 & -0.09 & -0.18 \\
HOMO & 116 & 0.05 & -0.08 & -0.16 & 0.07 & -0.09 & -0.15 \\
LUMO & 117 & 0.19 & 0.05 & -0.06 & 0.20 & 0.07 & -0.07 \\
& 118 & 0.12 & 0.06 & -0.07 & 0.20 & 0.08 & -0.06 \\
& 119 & 0.20 & 0.08 & -0.05 & 0.22 & 0.09 & -0.05 \\
$\Delta \varepsilon(117-116)$ & & & & & & & \\
& & 0.14 & 0.13 & 0.08 & 0.13 & 0.15 & 0.09 \\
& & & & & & & \\
& 157 & 0.02 & -0.10 & -0.18 & 0.02 & -0.10 & -0.18 \\
& 158 & 0.05 & -0.10 & -0.18 & 0.05 & -0.10 & -0.18 \\
& 159 & 0.05 & -0.08 & -0.15 & 0.07 & -0.08 & -0.15 \\
& 160 & 0.13 & 0.05 & -0.07 & 0.13 & 0.06 & -0.07 \\
& 161 & 0.13 & 0.05 & -0.06 & 0.13 & 0.06 & -0.06 \\
& 162 & 0.14 & 0.06 & 0.05 & 0.14 & 0.07 & -0.05 \\
& & & & & & & \\
& & 0.08 & 0.13 & 0.09 & 0.06 & 0.14 & 0.09
\end{tabular}


Tabelle 2.27: Orbitalkoeffizienten für $\left[\mathrm{MO}(\mathrm{prz})_{2}\right]^{z-}(\mathrm{M}=\mathrm{Mo}, \mathrm{W} ; \mathrm{z}=-2,-1,0)$

\begin{tabular}{cccccc}
\hline & $\mathrm{M}(1)\left(\mathrm{d}_{x^{2} y^{2}}\right)$ & $\mathrm{S}(2)\left(\mathrm{p}_{z}\right)$ & $\mathrm{S}(3)\left(\mathrm{p}_{z}\right)$ & $\mathrm{S}(4)\left(\mathrm{p}_{z}\right)$ & $\mathrm{S}(5)\left(\mathrm{p}_{z}\right)$ \\
\hline $\mathbf{7 a}$ & 0.73046 & $0.02752\left(\mathrm{p}_{y}\right)$ & -0.02605 & -0.02753 & 0.02594 \\
$\mathbf{7 b}$ & 0.33589 & -0.09999 & -0.10147 & 0.09483 & 0.09763 \\
$\mathbf{7 c}$ & 0.43744 & -0.08990 & -0.09036 & 0.07234 & 0.07673 \\
$\mathbf{8 a}$ & 0.70374 & $0.02318\left(\mathrm{p}_{y}\right)$ & -0.02114 & -0.02324 & 0.02114 \\
$\mathbf{8 b}$ & 0.35431 & -0.09973 & -0.10246 & 0.08801 & 0.09035 \\
$\mathbf{8 c}$ & 0.52271 & 0.08498 & 0.08351 & -0.07040 & -0.07095 \\
& & & & & \\
\hline & $\mathrm{C}(6)\left(\mathrm{p}_{z}\right)$ & $\mathrm{C}(7)\left(\mathrm{p}_{z}\right)$ & $\mathrm{C}(10)\left(\mathrm{p}_{z}\right)$ & $\mathrm{C}(11)\left(\mathrm{p}_{z}\right)$ & $\mathrm{O}(17)\left(\mathrm{p}_{x}\right)$ \\
\hline $\mathbf{7 a}$ & $0.06155\left(\mathrm{p}_{x}\right)$ & 0.04744 & -0.04688 & -0.06099 & $0.01547\left(\mathrm{p}_{z}\right)$ \\
$\mathbf{7 b}$ & 0.09319 & 0.09666 & -0.11943 & -0.12237 & 0.07885 \\
$\mathbf{7 c}$ & 0.07919 & 0.08003 & -0.09988 & -0.09770 & 0.05962 \\
$\mathbf{8 a}$ & $0.07168\left(\mathrm{p}_{x}\right)$ & 0.05619 & -0.07167 & -0.05617 & $0.01021\left(\mathrm{p}_{z}\right)$ \\
$\mathbf{8 b}$ & $0.08055\left(\mathrm{p}_{x}\right)$ & -0.06163 & 0.05811 & -0.04827 & 0.05957 \\
$\mathbf{8 c}$ & -0.06365 & -0.06163 & 0.09835 & 0.09622 & -0.05445
\end{tabular}

Bei den Molybdopterinkomplexen ist die HOMO-LUMO-Lücke in OS IV für Mo und $\mathrm{W}$ ist mit $0.08 / 0.06$ Hartree (W) nur noch fast halb so groß wie die der Pyrazinkomplexe. In OS V und VI wiederum sind die Werte beider Systeme gleich groß.

Die Orbitalkoeffizienten (Tab. 2.27) und 2.28) zeigen dagegen einen höheren MetallAnteil der Mo-Komplexe in OS IV mit $73 \%$ Mo- vs $70 \%$ W-Anteil (Pyrazin) und insgesamt einem geringeren Metallanteil im Molybdopterin mit 63\% Mo- und 61\% W-Anteil. In den OS V und VI ist der Metallanteil beim Wolfram höher, allerdings sind die Unterschiede zwischen Molybdän und Wolfram geringer als bei den anderen Systemen.

Wieder ist ein Absinken des Metallanteils in OS V und ein erneuter Anstieg in OS VI zu beobachten. Der Anteil des Schwefels ist nun auch bei den Molybdänkomplexen in OS IV wesentlich geringer und beträgt nur noch 2-3\%, während der der C-Atome zwischen 4 und 6\% liegt. Die Ausrichtung der bindenden p-Orbitale des Schwefels erfolgt hier auch entlang der $\mathrm{y}$-Achse $\left(\mathrm{p}_{y}\right)$, da so eine bessere Überlappung gewährleistet ist. 
Tabelle 2.28: Orbitalkoeffizienten für $\left[\mathrm{MO}(\mathrm{mpt})_{2}\right]^{z-}(\mathrm{M}=\mathrm{Mo}, \mathrm{W} ; \mathrm{z}=-2,-1,0)$

\begin{tabular}{cccccc}
\hline & $\mathrm{M}(1)\left(\mathrm{d}_{x^{2} y^{2}}\right)$ & $\mathrm{S}(2)\left(\mathrm{p}_{z}\right)$ & $\mathrm{S}(3)\left(\mathrm{p}_{z}\right)$ & $\mathrm{S}(4)\left(\mathrm{p}_{z}\right)$ & $\mathrm{S}(5)\left(\mathrm{p}_{z}\right)$ \\
\hline $\mathbf{9 a}$ & 0.63155 & $-0.02567\left(\mathrm{p}_{y}\right)$ & 0.02445 & 0.02568 & -0.02444 \\
$\mathbf{9 b}$ & 0.25551 & -0.10472 & -0.10443 & 0.09501 & 0.09707 \\
$\mathbf{9 c}$ & 0.38787 & -0.09261 & -0.08196 & 0.07687 & 0.06659 \\
$\mathbf{1 0 a}$ & 0.60981 & $-0.02227\left(\mathrm{p}_{y}\right)$ & 0.01942 & 0.02226 & -0.01942 \\
$\mathbf{1 0 b}$ & 0.29122 & -0.10334 & -0.10422 & 0.09230 & 0.09421 \\
$\mathbf{1 0 c}$ & 0.44459 & -0.08931 & -0.08137 & 0.07010 & 0.06478 \\
& & & & & \\
\hline & $\mathrm{C}(6)\left(\mathrm{p}_{z}\right)$ & $\mathrm{C}(7)\left(\mathrm{p}_{z}\right)$ & $\mathrm{C}(10)\left(\mathrm{p}_{z}\right)$ & $\mathrm{C}(11)\left(\mathrm{p}_{z}\right)$ & $\mathrm{O}(17)\left(\mathrm{p}_{x}\right)$ \\
\hline $\mathbf{9 a}$ & $-0.06554\left(\mathrm{p}_{x}\right)$ & -0.04080 & 0.06551 & 0.04077 & $0.01279\left(\mathrm{p}_{z}\right)$ \\
$\mathbf{9 b}$ & 0.09860 & 0.10007 & -0.11836 & -0.11900 & -0.06087 \\
$\mathbf{9 c}$ & 0.07674 & 0.06586 & -0.10067 & -0.08933 & -0.04431 \\
$\mathbf{1 0 a}$ & $-0.07597\left(\mathrm{p}_{x}\right)$ & -0.04844 & 0.07597 & 0.04844 & $0.00845\left(\mathrm{p}_{z}\right)$ \\
$\mathbf{1 0 b}$ & 0.09793 & 0.09909 & -0.11506 & -0.11559 & -0.06042 \\
$\mathbf{1 0 c}$ & 0.06774 & 0.05906 & -0.09466 & -0.08639 & 0.04006
\end{tabular}

Die Betrachtung der S-S-Bindung ergibt für beide Systeme unterschiedliche Ergebnisse. Bei der Pyrazinverbindung fehlen im Molybdänkomplex in OS IV sämtliche antibindenden Wechselwirkungen, so dass bei beiden Liganden eine hohe Bindungsordnung von 0.88 auftritt. Bei der entsprechenden Wolframverbindung kommt eine Besetzung des antibindenden Orbitals hinzu, die die Bindung auf 0.06 abschwächt. In OS VI zeigt sich bei der Molybdänverbindung nur auf der gefalteten Seite eine Wechselwirkung von 0.14 und bei der analogen Wolframverbindung sind beide Seiten antibindend. Beim Molybdopterinsystem dagegen gibt es in OS IV sowohl bei den Molybdän- als auch bei den Wolframkomplexen nur eine schwache Interaktion mit Werten für die Bindungsordnung von 0.07/0.06, wohingegen in OS VI nur der Molybdänkomplex über eine bindende Wechselwirkung mit einer Bindungsordnung von 0.15 auf der Seite des ungefalteten Liganden verfügt. 
Tabelle 2.29: Wechselwirkungen der Schwefel-Atome

\begin{tabular}{|c|c|c|c|c|c|}
\hline A-B & Besetzung & $\mathrm{BO}^{a}$ & A-B & Besetzung & $\mathrm{BO}$ \\
\hline \multicolumn{3}{|c|}{$\left[\mathrm{Mo}^{I V} \mathrm{O}(\mathrm{prz})_{2}\right]^{2-}$} & \multicolumn{3}{|c|}{$\left[\mathrm{Mo}^{I V} \mathrm{O}(\mathrm{mpt})_{2}\right]^{2-}$} \\
\hline $\mathrm{S}(2)-\mathrm{S}(3)$ & 1.75 & 0.88 & $\mathrm{~S}(2)-\mathrm{S}(3)$ & 1.82 & 0.07 \\
\hline $\mathrm{S}(2)-\mathrm{S}(3) * b$ & & & $\mathrm{~S}(2)-\mathrm{S}(3)^{*}$ & 1.67 & \\
\hline $\mathrm{S}(4)-\mathrm{S}(5)$ & 1.75 & 0.88 & $\mathrm{~S}(4)-\mathrm{S}(5)$ & 1.82 & 0.07 \\
\hline $\mathrm{S}(4)-\mathrm{S}(5)^{*}$ & & & $\mathrm{~S}(4)-\mathrm{S}(5)^{*}$ & 1.67 & \\
\hline \multicolumn{3}{|c|}{$\left[\mathrm{Mo}^{V I} \mathrm{O}(\mathrm{prz})_{2}\right]$} & \multicolumn{3}{|c|}{$\left[\mathrm{Mo}^{V I} \mathrm{O}(\mathrm{mpt})_{2}\right]$} \\
\hline $\mathrm{S}(2)-\mathrm{S}(3)$ & 1.34 & $(-0.17)$ & $\mathrm{S}(2)-\mathrm{S}(3)$ & 1.67 & 0.16 \\
\hline $\mathrm{S}(2)-\mathrm{S}(3)^{*}$ & 1.68 & & $\mathrm{~S}(2)-\mathrm{S}(3)^{*}$ & 1.36 & \\
\hline $\mathrm{S}(4)-\mathrm{S}(5)$ & 1.63 & 0.14 & $\mathrm{~S}(4)-\mathrm{S}(5)$ & 1.45 & $(-0.12)$ \\
\hline $\mathrm{S}(4)-\mathrm{S}(5)^{*}$ & 1.35 & & $\mathrm{~S}(4)-\mathrm{S}(5)^{*}$ & 1.68 & \\
\hline \multicolumn{3}{|c|}{$\left[\mathrm{W}^{I V} \mathrm{O}(\mathrm{prz})_{2}\right]^{2-}$} & \multicolumn{3}{|c|}{$\left[\mathrm{W}^{I V} \mathrm{O}(\mathrm{mpt})_{2}\right]^{2-}$} \\
\hline $\mathrm{S}(2)-\mathrm{S}(3)$ & 1.80 & 0.06 & $\mathrm{~S}(2)-\mathrm{S}(3)$ & 1.80 & 0.06 \\
\hline $\mathrm{S}(2)-\mathrm{S}(3)^{*}$ & 1.69 & & $\mathrm{~S}(2)-\mathrm{S}(3)^{*}$ & 1.68 & \\
\hline $\mathrm{S}(4)-\mathrm{S}(5)$ & 1.80 & 0.06 & $\mathrm{~S}(4)-\mathrm{S}(5)$ & 1.80 & 0.06 \\
\hline $\mathrm{S}(4)-\mathrm{S}(5)^{*}$ & 1.69 & & $\mathrm{~S}(4)-\mathrm{S}(5)^{*}$ & 1.68 & \\
\hline \multicolumn{3}{|c|}{$\left[\mathrm{W}^{V I} \mathrm{O}(\mathrm{prz})_{2}\right]$} & \multicolumn{3}{|c|}{$\left[\mathrm{W}^{V I} \mathrm{O}(\mathrm{mpt})_{2}\right]$} \\
\hline $\mathrm{S}(2)-\mathrm{S}(3)$ & 1.35 & $(-0.17)$ & $\mathrm{S}(2)-\mathrm{S}(3)$ & - & - \\
\hline $\mathrm{S}(2)-\mathrm{S}(3)^{*}$ & 1.69 & & $\mathrm{~S}(2)-\mathrm{S}(3)^{*}$ & - & \\
\hline $\mathrm{S}(4)-\mathrm{S}(5)$ & 1.46 & $(-0.12)$ & $\mathrm{S}(4)-\mathrm{S}(5)$ & 1.47 & $(-0.11)$ \\
\hline $\mathrm{S}(4)-\mathrm{S}(5)^{*}$ & 1.70 & & $\mathrm{~S}(4)-\mathrm{S}(5)^{*}$ & 1.69 & \\
\hline
\end{tabular}

\subsection{Ringöffnung}

Hinsichtlich der diskutierten Möglichkeit des Molybdopterins von der reduzierten trizyklischen Tetrahydropteridin-Form durch Öffnung des Pyranringes in die DihydropteridinForm überzugehen, wurden Modelle dieser Form berechnet. Um der mechanistischen Problematik gerecht zu werden und die den Pyransauerstoff umgebenen Atome analog zum natürlichen Kofaktor zu belassen, wurde als Modell die Pyrazinverbindung gewählt, welche mit ihrem Pyrazinring die protonierten Stickstoffe zur Verfügung stellt aber aufgrund der kleineren Größe gegenüber der Molybdopterinverbindung weniger Rechenaufwand benötigt. 
Tabelle 2.30: Ausgewählte Geometrieparameter $\left[\mathrm{Mo}^{I V} \mathrm{O}(\mathrm{prz})_{2}\right]^{2-}$

\begin{tabular}{lcccccc}
\hline & $\mathbf{7 a}$ & $\mathbf{7 b}$ & $\mathbf{7 c}$ & $\mathbf{8 a}$ & $\mathbf{8 b}$ & $\mathbf{8 c}$ \\
\hline $\mathrm{C}(13)-\mathrm{O}(8)$ & 1.4361 & 1.4238 & 1.4165 & 1.4368 & 1.42389 & 1.4145 \\
$\mathrm{O}(8)-\mathrm{C}(15)$ & 1.4236 & 1.4289 & 1.4291 & 1.4239 & 1.42910 & 1.4328 \\
$\mathrm{C}(19)-\mathrm{O} 21)$ & 1.4361 & 1.4241 & 1.4153 & 1.4369 & 1.4239 & 1.4135 \\
$\mathrm{O}(21)-\mathrm{C}(22)$ & 1.4236 & 1.4286 & 1.4319 & 1.4240 & 1.4291 & 1.4324 \\
$\mathrm{C}(7)-\mathrm{C}(6)-\mathrm{C}(13)-\mathrm{O}(8)$ & -10.85 & -12.95 & -16.20 & -10.68 & -12.25 & -14.83 \\
$\mathrm{C}(7)-\mathrm{C}(14)-\mathrm{C}(15)-\mathrm{O}(8)$ & 50.99 & 51.74 & 51.28 & 50.96 & 51.78 & 51.92 \\
$\mathrm{C}(10)-\mathrm{C}(11)-\mathrm{C}(19)-\mathrm{O}(21)$ & -10.85 & -10.11 & -14.60 & -10.68 & -9.81 & -12.00 \\
$\mathrm{C}(10)-\mathrm{C}(20)-\mathrm{C}(22)-\mathrm{O}(21)$ & 50.99 & 52.19 & 51.02 & 50.96 & 52.30 & 51.72
\end{tabular}

Bisher ist nicht bekannt, unter welchen Bedingungen bzw. bei welchem Schritt der Oxotransferreaktion eine Ringöffnung eintreten könnte. Ebensowenig ist der genaue Mechanismus geklärt.

Um eine möglich Neigung zur Öffnung innerhalb der geschlossen Formen in verschiedenen OS zu erkennen sind die dafür in Betracht kommenden C-O-Bindungslängen des Pyranrings der Pyrazinverbindungen in Tabelle 2.30 dargestellt.

Mit zunehmender OS verkürzen sich wie oben besprochen die C-O-Bindungen neben der C-C-Doppelbindung (C(13)-O(8) und C(19)-O(21)) während sich die C-OBindung auf der anderen Seite nur sehr gering verlängert. Der Sauerstoff wird also in Richtung der Dithioleneinheit gezogen, was auch mit der von Bertero et al. [18] gefundenen geöffneten Struktur im Protein übereinstimmt, bei der der Sauerstoff auf dieser Seite verbleibt.

Weiterhin vergrößert sich der Diederwinkel C(7)-C(6)-C(13)-O(8) um etwa $5^{\circ}$ (Mo) bzw. $4^{\circ}(\mathrm{W})$, so dass das Sauerstoff-Atom weiter aus der Ebene herausgehoben wird. Die Bindungsverkürzung in Richtung der Ditholeneinheit zeigt deutlich die elektronenziehende Wirkung seitens des Metalls, welche sich bis hierher, wenn auch in geringem Ausmaß, auswirkt. Das Herausheben des Sauerstoffatoms ist eine Folge dieser Verkürzung, um so die auftretenden Abstoßungskräfte zu minimieren. Der Pyranring kann 
Abbildung 2.21: Struktur der offenen Pyrazinverbindungen

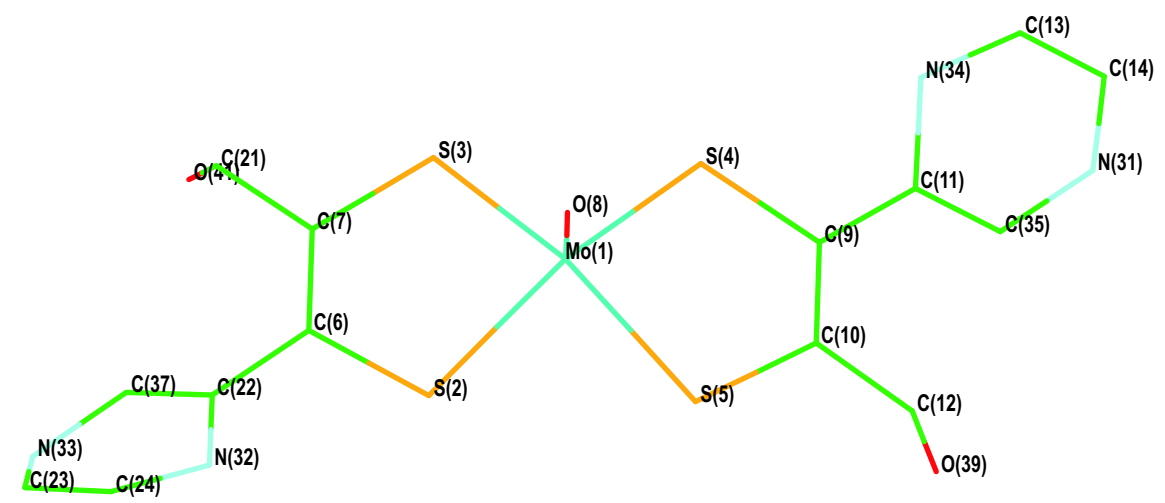

also mit einer gewissen Flexibilität auf die elektronische Situation reagieren.

Die Modellkomplexe der offenen Pyrazinverbindungen wurden daher mit beiden Metallen in allen relevanten OS (IV-VI) berechnet. Abbildung 2.21 zeigt die Struktur einer exemplarischen Verbindung und in Tabelle 2.31 sind die wichtigsten Geometrieparameter zusammengestellt.

Die Nomenklatur ist dabei wie folgt: 7d-f bezeichnet die Verbindungen $\left[\mathrm{MoO}(\mathrm{prz})_{2}\right]^{z-}$ mit $\mathrm{z}=-2,-1,0 ; \mathbf{8 d - f}$ bezeichnet die Verbindungen $\left[\mathrm{WO}(\mathrm{prz})_{2}\right]^{z-}$ mit $\mathrm{z}=-2,-1,0$.

Eine deutliche Übereinstimmung findet sich bei der M-Oxo-Bindungslänge die ihrem geschlossenen Homologen entspricht. Unterschiede fallen jedoch bereits bei Betrachtung der Me-S-Bindungen auf, die in der offenen Form um durchschnittlich 0.02 A deutlich kürzer sind. Die Unterschiede zwischen den vier Schwefel-Atomen innerhalb eines Moleküls folgen dem gleichen Trend wie in der geschlossenen Form, jedoch ist dieser Trend bei den Molybdänverbindungen etwas stärker und bei den Wolframverbindungen etwas schwächer ausgeprägt. So sind wiederum die S-Atome, die zu einem Liganden gehören von unterschiedlicher Länge (Mo-S(2)/Mo-S(4) $2.454 \AA$; MoS(3)/Mo-S(5) 2.449 §), jedoch gleichlang wie die trans-ständigen M-S-Bindungen des Liganden gegenüber. Die M-S-Bindungen nehmen mit zunehmender OS ab, beim Mo von durchschnittlich $2.45 \AA$ auf $2.43 \AA$ und beim W von $2.43 \AA$ auf $2.40 \AA$. 
Tabelle 2.31: Ausgewählte Geometrieparameter der offenen Form $\left[\mathrm{MO}(\mathrm{prz})_{2}\right]^{z-}$

\begin{tabular}{lcccccc}
\hline & $\mathbf{7 d}^{a}$ & $\mathbf{7 e}$ & $\mathbf{7 f}$ & $\mathbf{8 d}^{b}$ & $\mathbf{8 e}$ & $\mathbf{8 f}$ \\
\hline $\mathrm{M}(1)-\mathrm{S}_{4}$ & 0.7743 & 0.8173 & 0.85011 & 0.7452 & 0.7747 & 0.7902 \\
$\mathrm{M}(1)-\mathrm{O}(8)$ & 1.7141 & 1.7080 & 1.69952 & 1.7245 & 1.7157 & 1.7095 \\
$\mathrm{M}(1)-\mathrm{S}(2)$ & 2.4539 & 2.4452 & 2.4256 & 2.4344 & 2.4350 & 2.4139 \\
$\mathrm{M}(1)-\mathrm{S}(3)$ & 2.4490 & 2.4411 & 2.4287 & 2.4323 & 2.4325 & 2.3990 \\
$\mathrm{M}(1)-\mathrm{S}(4)$ & 2.4539 & 2.4203 & 2.4253 & 2.4344 & 2.4176 & 2.4113 \\
$\mathrm{M}(1)-\mathrm{S}(5)$ & 2.4490 & 2.4245 & 2.4324 & 2.4323 & 2.4223 & 2.4070 \\
$\mathrm{~S}(2)-\mathrm{C}(6)$ & 1.8030 & 1.7599 & 1.7777 & 1.8128 & 1.7692 & 1.7828 \\
$\mathrm{~S}(3)-\mathrm{C}(7)$ & 1.7822 & 1.7619 & 1.7320 & 1.7900 & 1.7709 & 1.7356 \\
$\mathrm{~S}(4)-\mathrm{C}(9)$ & 1.8030 & 1.7941 & 1.7819 & 1.8128 & 1.7979 & 1.7843 \\
$\mathrm{~S}(5)-\mathrm{C}(10)$ & 1.7822 & 1.7787 & 1.74263 & 1.7900 & 1.7842 & 1.7543 \\
$\mathrm{C}(6)-\mathrm{C}(7)$ & 1.3591 & 1.3560 & 1.3863 & 1.3559 & 1.3506 & 1.3802 \\
$\mathrm{C}(9)-\mathrm{C}(10)$ & 1.3591 & 1.3609 & 1.3876 & 1.3559 & 1.3587 & 1.3815
\end{tabular}

${ }^{a} \mathbf{7 d - f}$ bezeichnen die offenen Formen der Verbindungen $\left[\mathrm{MoO}(\mathrm{prz})_{2}\right]^{z}, \mathrm{z}=-2,-1,0$;

${ }^{b}$ 8d-f bezeichnen die offenen Formen der Verbindungen $\left[\mathrm{WO}(\mathrm{prz})_{2}\right]^{z}, \mathrm{z}=-2,-1,0$

Die benachbarten S-C-Bindungen schwanken wesentlich stärker beim Vergleich der an einem Liganden liegenden Bindungen sowie beim Wechsel der Oxidationsstufen. So sind sie teils viel länger als im geschlossenen Homologen und teilweise gleichlang. In den OS IV und VI ist wieder der Effekt zu beobachten, dass zwei zu einem Liganden gehörende Bindungen unterschiedlich lang sind, so z.B. S(2)-C(6) und S(4)$\mathrm{C}(7)$ mit $1.803 \AA, \mathrm{S}(3)-\mathrm{C}(7)$ und $\mathrm{S}(5)-\mathrm{C}(10)$ mit $1.782 \AA$ und zwar als Reaktion auf die Bindungslängenänderung der M-S-Bindung (längere M-S-Bindung, längere C-SBindung). In OS V dagegen sind alle Bindungen ohne erkennbaren Trend unterschiedlich lang. Aufgrund dieser Schwankungen kann nicht eindeutig von einem Absinken der Bindungslänge mit zunehmender Oxidation gesprochen werden.

Die anschließende C-C-Doppelbindung ist in den OS IV und V um etwa $0.01 \AA$ und in der OS VI um $0.02 \AA$ länger als in der geschlossenen Form. Die Werte der sich gegenüberliegenden Bindungen unterscheiden sich wenig bis gar nicht. Die Differenz steigt mit zunehmender Oxidation erst in OS VI um fast $0.03 \AA$ an. 
Tabelle 2.32: Weitere Geometrieparameter der offenen Form $\left[\mathrm{MO}(\mathrm{prz})_{2}\right]^{z-}$

\begin{tabular}{lcccccc}
\hline & $\mathbf{7 d}$ & $\mathbf{7 e}$ & $\mathbf{7 f}$ & $\mathbf{8 d}$ & $\mathbf{8 e}$ & $\mathbf{8 f}$ \\
\hline $\mathrm{C}(12)-\mathrm{O}(17)$ & 1.4550 & 1.4383 & 1.4386 & 1.4550 & 1.4381 & 1.4406 \\
$\mathrm{C}(8)-\mathrm{O}(17)$ & 1.4550 & 1.4135 & 1.4391 & 1.4550 & 1.4124 & 1.4413 \\
$\mathrm{~S}(2)-\mathrm{S}(3)$ & 3.2021 & 3.2216 & 3.1756 & 3.1862 & 3.2227 & 3.1578 \\
$\mathrm{~S}(4)-\mathrm{S}(5)$ & 3.2024 & 3.1705 & 3.1922 & 3.1862 & 3.1768 & 3.1881 \\
$\mathrm{C}(6)-\mathrm{C}(22)$ & 1.4738 & 1.5184 & 1.4416 & 1.4716 & 1.5174 & 1.4420 \\
$\mathrm{C}(9)-\mathrm{C}(11)$ & 1.4738 & 1.4737 & 1.4483 & 1.4716 & 1.4750 & 1.4558 \\
$\mathrm{O}(8)-\mathrm{M}(1)-[\mathrm{S}(2) \mathrm{S}(3)]$ & 114.54 & 118.23 & 117.88 & 113.68 & 116.62 & 118.17 \\
$\mathrm{O}(8)-\mathrm{M}(1)-[\mathrm{S}(4) \mathrm{S}(5)]$ & 114.54 & 115.23 & 115.55 & 113.68 & 114.18 & 113.55 \\
$\mathrm{FW} \mathrm{S}(2)-\mathrm{S}(3)$ & 15.92 & 13.39 & 1.51 & 14.54 & 7.89 & 11.9 \\
$\mathrm{FW} \mathrm{S}(4)-\mathrm{S}(5)$ & 15.85 & 29.51 & 23.88 & 14.54 & 29.96 & 34.77 \\
$\mathrm{C}(7)-\mathrm{C}(6)-\mathrm{C}(22)-\mathrm{C}(24)$ & -121.68 & 113.46 & -164.73 & -121.60 & 114.29 & -164.79 \\
$\mathrm{C}(10)(\mathrm{C} 9) \mathrm{C}(11)-\mathrm{C}(13)$ & -121.70 & 100.25 & -167.61 & -121.61 & 101.84 & -171.14
\end{tabular}

Die C-O-Bindung, des jetzt zum endständigen Alkohol gewordenen SauerstoffAtoms ist mit $1.45 \AA$ in OS IV nur unwesentlich länger als im Pyranring mit $1.44 \AA$. Wieder ist im Laufe der Oxidation eine geringe Abnahme der Bindungslänge um 0.01 bis $0.02 \AA \mathrm{zu}$ beobachten.

Ein ganz wesentlicher Unterschied ist im S-S-Abstand, der zu einem Liganden gehörenden Schwefel-Atome zu sehen, der in der offenen Form mit 3.2021/3.1756 ^ (Mo/W OS IV) viel kürzer als in der geschlossenen Form ist $(3.2611 / 3.2387 \AA \mathrm{Mo} / \mathrm{W}$ OS IV). Beim Wechsel der OS verhalten sich beide Liganden unterschiedlich. Der S(2)-S(3) Abstand im nicht gefalteten Liganden erhöht sich in OS V auf $3.2216 \AA$ (Mo, W auf $3.2227 \AA$ ) und verringert sich wieder in OS VI (Mo $3.1756 \AA$, W 3.1578 $\AA$ ). Die Bindungslänge S(4)-S(5) dagegen verringert sich in OS V auf 3.1705/3.1768 A nur wenig und steigt dann in OS VI wiederum nur um einen kleinen Betrag an (3.1922/3.1881 § Mo/W). Durch das Wegfallen des starren Ringsystems bietet sich nun eine etwas größere Flexibilität für die vorher durch den Dithiolenchelatring stark beschränkte Geometrie.

Ein interessantes Verhalten zeigen die C-C-Doppelbindungen am Pyrazinring C(6)- 
$\mathrm{C}(22)$ bzw. C(9)-C(11). Beide Metalle weisen die gleichen Veränderungen und ähnliche Werte auf, wobei die Längen am W marginal kürzer ausfallen. In OS IV betragen die Bindungslängen bei Mo und W auf beiden Seiten des Moleküls je $1.4738 \AA(\mathrm{Mo})$. Die C-C-Bindung im nicht gefalteten Liganden $\mathrm{C}(6)-\mathrm{C}(22)$ steigt beim Wechsel in die OS $\mathrm{V}$ auf $1.5184 \AA$ an und sinkt dann in OS VI wieder ab auf $1.4416 \AA$, etwa $0.03 \AA$ unter ihren Ausganswert in OS IV. Die C-C-Bindung im gefalteten Liganden C(9)$\mathrm{C}(11)$ dagegen bleibt beim Wechsel in die OS IV nahezu konstant (1.4737 $\AA$ ) und sinkt dann auf $1.4483 \AA$ in OS VI ab.

Ein weiterer Unterschied zeigt sich bei den Werten des Faltungswinkels. Beim Wechsel in OS V steigt er bei der Molybdänverbindung auf $29.51^{\circ}$ analog zu der geschlossenen Form woraufhin in der OS VI ein Absinken auf $23.88^{\circ}$ erfolgt, während bei der Wolframverbindung beide Male ein Anheben erfolgt, einmal von $14.54^{\circ}$ auf $29.96^{\circ}$ in OS V und ein weiteres Mal auf $34.77^{\circ}$ in OS VI.

Um die analog zu den in den geschlossenen Homologen auftretende Abwinkelung des Pyrazinringes zu beschreiben wurden die Diederwinkel $\mathrm{C}(7)-\mathrm{C}(6)-\mathrm{C}(22)-\mathrm{C}(24)$ für den Ring am nicht gefalteten Liganden und C(10)-C(9)-C(11)-C(13) für den Ring am gefalteten Liganden gewählt. Bei beiden Metallen verhalten sich die Pyrazinringe sehr ähnlich (Abweichung $<2^{\circ}$ ) jedoch finden sich große Unterschiede beim Wechsel der OS und beim Vergleich beider Liganden.

So ist der Diederwinkel in OS IV auf beiden Seiten je gleich groß (ca. 122 $2^{\circ}$, Mo und $\mathrm{W}$, links und rechts 15 ) in OS V sind bereits Unterschiede von $3^{\circ}$ (Mo: $113.46^{\circ}$ links und $100.25^{\circ}$ rechts, W: $114.29^{\circ}$ links, $101.84^{\circ}$ rechts) und in OS VI von 3 bzw. $7^{\circ}$ (Mo: $164.73^{\circ}$ links und $167.61^{\circ}$ rechts, W: $164.79^{\circ}$ links und $171.14^{\circ}$ rechts) zu sehen. Erstaunlich ist die enorme Änderung dieses Winkels insgesamt beim Wechsel der OS von $122^{\circ}$ in OS IV auf $100 / 113^{\circ}$ in OS V und $164 / 167^{\circ}$ in OS VI (Mo). Die frei

\footnotetext{
${ }^{15}$ Auf der linken Seite befindet sich der nicht gefaltete Ligand, auf der rechten der gefaltete.
} 
rotierbare Einfachbindung über die der Pyrazinring an die Dithioleneinheit gebunden ist, lässt eine sehr große Flexibilität des Ringes zu.

Die am Metallzentrum beobachteten Änderungen der Bindungslängen spiegeln die bereits in den geschlosssenen Formen analysierten Trends mit zunehmender Oxidation deutlich wieder und treten sogar verstärkt auf, was sich an den bereits dargelegten Änderungen der Bindungslängen zeigt. Die gewonnene Flexibilität durch den Wegfall des Pyranrings, dessen Ringstruktur eine sterische Einschränkung für die an die C-C-Doppelbindung geknüpften Atome darstellt, scheint damit die Beweglichkeit von Ladungen zu fördern. Der Wegfall der Verbindung zum Pteridingerüst über das an den Pyransauerstoff gebundene C-Atom hat somit keinen großen Einfluss auf die elektronischen Abläufe während des Redoxprozesses. Wie auch bei den geschlossenen Formen beobachtet, trägt der Pyransauerstoff nicht zur Konjugation, also zur elektronischen Verbindung mit dem Pteridingerüst, bei. Auch damit wäre die in der offenen Form verbleibende einzige Bindung zum Pyrazinring ausreichend, um den Einfluss des Liganden auf die Dithioleneinheit zu ermöglichen.

Für die Abschätzung der energetischen Machbarkeit der Ringöffnung wurden die Energiedifferenzen der offenen gegenüber den geschlossenen Formen berechnet. Die reinen ab initio-Energien sowie deren Differenzbeträge in $\mathrm{kJ} / \mathrm{mol}$ sind in Tabelle 2.33 dargestellt. Weiterhin sind auch die Unterschiede zwischen den einzelnen OS aufgeführt.

Anhand der Energien ist erkennbar, dass alle Modellkomplexe der offenen Form energetisch höher liegen als ihre geschlossenen Homologen. Die Energien unterscheiden sich dabei beim Wechsel der OS sowie beim Wechsel des Metalls. In den OS IV und $\mathrm{V}$ betragen die Differenzen zwischen offener und geschlossener Form für die Molybdän- und Wolframverbindungen 129.98/125.56 kJ/mol (Mo/W, IV) und 267.83/268.01 kJ/mol (Mo/W, V). In Oxidationsstufe VI sind bei beiden Metallen 
Tabelle 2.33: Energien und Energiedifferenzen der offenen und geschlossenen Formen

\begin{tabular}{lccccc}
\hline & geschlossen $^{a}$ & $\Delta \mathrm{E}_{\text {ox-red }}{ }^{b}$ & offen $^{a}$ & $\Delta \mathrm{E}_{x-\text { red }}{ }^{b}$ & $\Delta \mathrm{E}_{\text {offen-geschl. }}{ }^{b}$ \\
\hline $\left.\mathrm{Mo}^{I V} \mathrm{O}(\mathrm{prz})_{2}\right]^{2-}$ & -2648.57037 & & -2648.52086 & & \\
{$\left[\mathrm{Mo}^{V} \mathrm{O}(\mathrm{prz})_{2}\right]^{1-}$} & -2648.58923 & -49.53 & -2648.48722 & 88.32 & 129.98 \\
{$\left[\mathrm{Mo}^{V I} \mathrm{O}(\mathrm{prz})_{2}\right]$} & -2648.46849 & 317.00 & -2648.46329 & 62.84 & 13.66 \\
{$\left[\mathrm{~W}^{I V} \mathrm{O}(\mathrm{prz})_{2}\right]^{2-}$} & -2648.87322 & & -2648.82539 & & 125.56 \\
{$\left[\mathrm{~W}^{V} \mathrm{O}(\text { prz })_{2}\right]^{1-}$} & -2648.90212 & -75.90 & -2648.80004 & 66.56 & 268.01 \\
{$\left[\mathrm{~W}^{V I} \mathrm{O}(\mathrm{prz})_{2}\right]$} & -2648.78350 & 311.44 & -2648.76307 & 97.09 & 53.66 \\
& & & & \\
${ }^{a}$ Energien in Hartree, $1 \mathrm{Hartree}=2625.4999638 \mathrm{~kJ} / \mathrm{mol}$ & & & \\
${ }^{b}$ Energiedifferenzen in kJ/mol &
\end{tabular}

die Energieunterschiede signifikant niedriger, so betragen sie nur noch 13.66/53.66 $\mathrm{kJ} / \mathrm{mol}(\mathrm{Mo} / \mathrm{W})$.

Die Größenordnung dieser Werte zeigt, dass die geschlossenen Formen energetisch deutlich stabiler sind, es jedoch nicht unmöglich ist, dass während des katalytischen Prozesses eine Ringöffnung stattfinden könnte. Vor allem der in OS VI nur noch sehr geringe Energieunterschied vor allem bei der Molybdänverbindung von nur 13.66 $\mathrm{kJ} / \mathrm{mol}$, zeigt, dass die höhere Beweglichkeit des Ligandensystems den Elektronenmangel sehr gut auszugleichen vermag. Hinzu kommt, dass das im Enzym vorliegende Molybdopterin über zahlreiche H-Brücken mit dem Peptid verbunden ist, die in der Lage sind, diesen Energieaufwand abzufangen. Rechnungen zeigten, dass Protonierung eines der geöffneten Tautomere die Energie beim TS deutlich absenkt [132]. Auch ist es für das Pteridingerüst möglich, verschiedenen tautomere Formen anzunehmen, was eine weitere Flexibilität hinsichtlich des Elektronenflusses bedeutet.

Interessanterweise kehren sich die Energieunterschiede zwischen den einzelnen OS im Gegensatz zu den geschlossenen Homologen um. Die Verbindungen in OS IV sind nun um $88.32 / 66.56 \mathrm{~kJ} / \mathrm{mol}(\mathrm{Mo} / \mathrm{W})$ stabiler als die in OS V. Die Energiebarriere zur OS VI ist nun mit 62.84/97/09 kJ/mol deutlich erniedrigt, was bedeutet, dass der 
Oxidationsprozess insgesamt erleichtert wird.

Bei Ringschlussreaktionen zyklischer Ether allgemein stehen sich die Größen der auftretenden Ringspannung und des Entropieterms, der durch die Nähe der Reaktionszentren sowie die relative Starre des Übergangszustands beeinflusst wird, entgegen. Diese Faktoren ändern sich in Abhängigkeit von der Ringgröße [118]. Cyclohexane und ihre Derivate sind praktisch spannungsfrei jedoch überwiegt bei ihnen die große Entfernung der Alkoxidgruppe vom elektrophilen Kohlenstoff bzgl. des Entropieterms. Die Ringschlussreaktion ist daher durch ungünstige Entropieeffekte, verdeckte Konformationen und den damit verbundenen Spannungseffekten erschwert. Durch den Pyransauerstoff, welcher im Gegensatz zu einem aliphatischen C-Atom keinerlei HAtome trägt, wirkt er hinsichtlich der gauche-Wechselwirkungen spannungsmindernd. Diese Aspekte weisen auf die Möglichkeit eines reversiblen Gleichgewichts zwischen der offenen und geschlossenen Form hin.

Unter Berücksichtigung der bisher nicht betrachteten Einflüsse, die zur Stabilisierung des Systems herangezogen werden können, wie H-Brücken, Tautomerie oder Protonierungen, der durch das Wegfallen des Pyranrings erhöhten Freiheit des Liganden sowie der Abläufe am Metallzentrum, kann es in Enzymen während des katalytischen Prozesses durchaus zu einer Öffnung des Ringes kommen. Die Größenordungen der berechneten Energieunterschiede können durch Protonierung sowie durch den Einfluss des Lösungsmittels im Enzym aufgebracht werden, so dass sich ein Gleichgewicht zwischen beiden Formen herausbilden könnte. Einflüsse dieser Art bedürfen aber noch weitergehender Untersuchungen unter Berücksichigung der oben genannten Faktoren. 
Abbildung 2.22: Struktur der Produktverbindungen am Beispiel von $\left[\mathrm{MoO}_{2}(\mathrm{cdt})_{2}\right]^{2-}$

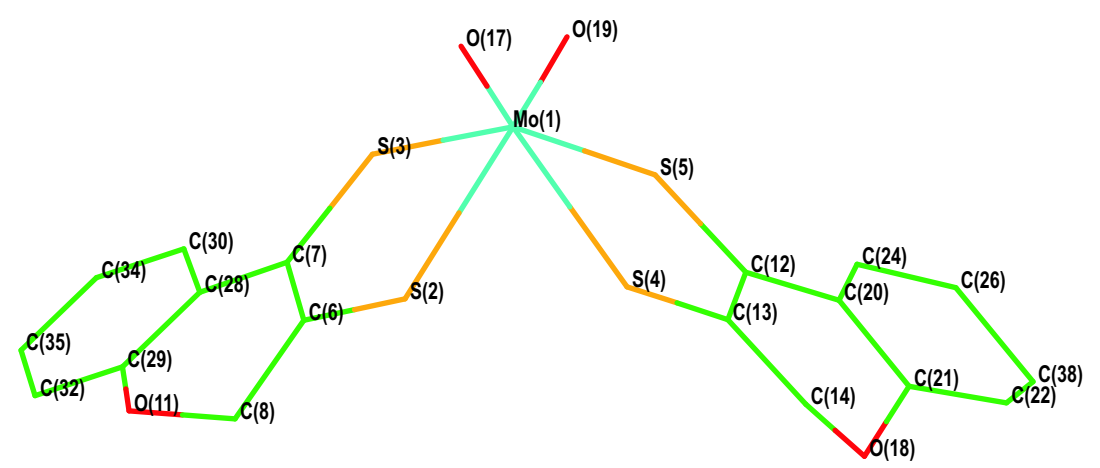

\subsection{Oxoaffinität}

Um einen besseren Einblick in die Energetik des Sauerstofftransfers zu bekommen, wurden für die bisher besprochenen Verbindungen die Energien der Sauerstoffübertragung berechnet. Die Modelle der Produktverbindungen beinhalten im Unterschied zu den Ausgangsverbindungen zwei Sauerstoffe am Metall.

Die Strukturen der Dioxoverbindungen unterscheiden sich recht stark von denen der ursprünglichen Monooxoverbindungen. Das Metall ist nun verzerrt oktaedrisch koordiniert, wobei ein Ligand orthogonal verdreht so verbleibt, dass ein Schwefel-Atom in der äquatorialen Ebenen liegt, während das andere die untere axiale Position einnimmt. Dies ist in Übereinstimmung mit kristallographisch untersuchten $\left[\mathrm{M}(\mathrm{dt})_{2} \mathrm{XY}\right]-$ Modellkomplexen $(\mathrm{dt}=$ dithiolenartiger Ligand, XY einzähnige Coliganden $)$, welche entweder zwei Oxo-Liganden bzw. einen monoanionischen Liganden wie z.B. Alkoxid, Siloxid oder Chlorid enthalten [133]. Alle diese Strukturen weisen eine verzerrt oktaedrische Geometrie auf.

Ein Sauerstoff-Atom steht in der oberen axialen Position, während das zweite mit in die äquatoriale Ebene eingebunden wird. Tabelle 2.34 zeigt die wichtigsten geometrischen Parameter; Abbildung 2.22 am Beispiel des Molybdänchromanylkomplexes die Struktur dieser Verbindungen. 
Tabelle 2.34: Ausgewählte Geometrieparameter der Produktverbindungen ${ }^{a}$

\begin{tabular}{|c|c|c|c|c|c|c|c|c|}
\hline & $1 \mathrm{~g}$ & $2 g$ & $3 g$ & $4 g$ & $7 g$ & $8 g$ & $9 g$ & $10 \mathrm{~g}$ \\
\hline $\mathrm{M}(1)-\mathrm{O}(17)$ & 1.7435 & 1.7457 & 1.7392 & 1.7419 & 1.7417 & 1.7443 & 1.7398 & 1.7426 \\
\hline $\mathrm{M}(1)-\mathrm{O}(19)$ & 1.7431 & 1.7456 & 1.7386 & 1.7414 & 1.7417 & 1.7443 & 1.7398 & 1.7426 \\
\hline $\mathrm{O}(17)-\mathrm{O}(19)$ & 2.7236 & 2.7127 & 2.7342 & 2.7244 & 2.7236 & 2.7138 & 2.7280 & 2.7149 \\
\hline $\mathrm{O}(17)-\mathrm{M}(1)-\mathrm{O}(19)$ & 102.73 & 101.97 & 103.66 & 102.91 & 102.86 & 102.14 & 103.26 & 102.34 \\
\hline $\mathrm{M}(1)-\mathrm{S}(2)$ & 2.7505 & 2.7355 & 2.7527 & 2.7405 & 2.7495 & 2.7366 & 2.7533 & 2.7399 \\
\hline $\mathrm{M}(1)-\mathrm{S}(3)$ & 2.5050 & 2.4965 & 2.4948 & 2.4890 & 2.5023 & 2.4944 & 2.4998 & 2.4920 \\
\hline $\mathrm{M}(1)-\mathrm{S}(4)$ & 2.7437 & 2.7344 & 2.7451 & 2.7317 & 2.7493 & 2.7365 & 2.7521 & 2.7391 \\
\hline $\mathrm{M}(1)-\mathrm{S}(5)$ & 2.5053 & 2.4968 & 2.4931 & 2.4865 & 2.5023 & 2.4944 & 2.4998 & 2.4922 \\
\hline $\mathrm{C}(6)-\mathrm{C}(7)$ & 1.3502 & 1.3482 & 1.3651 & 1.3634 & 1.3502 & 1.3484 & 1.3517 & 1.3498 \\
\hline $\mathrm{C}(12)-\mathrm{C}(13)$ & 1.3495 & 1.3479 & 1.3637 & 1.3621 & 1.3502 & 1.3484 & 1.3516 & 1.3498 \\
\hline $\mathrm{S}(2)-\mathrm{C}(6)$ & 1.7487 & 1.7513 & 1.7247 & 1.7267 & 1.7445 & 1.7466 & 1.7427 & 1.7450 \\
\hline $\mathrm{S}(3)-\mathrm{C}(7)$ & 1.7688 & 1.7731 & 1.7744 & 1.7784 & 1.7700 & 1.7745 & 1.7692 & 1.7736 \\
\hline $\mathrm{S}(4)-\mathrm{C}(13)$ & 1.7510 & 1.7528 & 1.7271 & 1.7292 & 1.7445 & 1.7467 & 1.7428 & 1.7450 \\
\hline $\mathrm{S}(5)-\mathrm{C}(12)$ & 1.7682 & 1.7727 & 1.7748 & 1.7786 & 1.7700 & 1.7745 & 1.7692 & 1.7736 \\
\hline $\mathrm{S}(2)-\mathrm{S}(3)$ & 3.3033 & 3.3112 & 3.3042 & 3.3119 & 3.3070 & 3.3138 & 3.3090 & 3.3171 \\
\hline $\mathrm{S}(4)-\mathrm{S}(5)$ & 3.3047 & 3.3124 & 3.3039 & 3.3082 & 3.3070 & 3.3138 & 3.3087 & 3.3170 \\
\hline $\mathrm{S}(2)-\mathrm{M}-\mathrm{S}(3)$ & 77.73 & 78.38 & 77.88 & 78.43 & 77.90 & 78.47 & 77.92 & 78.54 \\
\hline $\mathrm{S}(4)-\mathrm{M}-\mathrm{S}(5)$ & 77.89 & 78.43 & 78.05 & 78.53 & 77.90 & 78.45 & 77.94 & 78.55 \\
\hline $\mathrm{S}(2)-\mathrm{S}(3)-\mathrm{O}(19)-\mathrm{S}(5)$ & 7.99 & 6.99 & 6.52 & 6.50 & 6.60 & 6.23 & 6.67 & 6.21 \\
\hline $\mathrm{S}(2)-\mathrm{S}(3)-\mathrm{S}(4)-\mathrm{S}(5)$ & -53.72 & -53.81 & -54.59 & -54.98 & -53.51 & -53.52 & -53.28 & -53.43 \\
\hline $\mathrm{O}(17)-\mathrm{M}(1)-\mathrm{S}(4)$ & 160.26 & 161.98 & 160.52 & 161.78 & 160.2571 & 162.18 & 160.93 & 162.48 \\
\hline $\mathrm{O}(19)-\mathrm{M}(1)-\mathrm{S}(2)$ & 160.64 & 162.25 & 161.17 & 162.74 & 160.6423 & 162.19 & 160.89 & 162.51 \\
\hline
\end{tabular}

Zwischen den Systemen sowie den Molybdän- und Wolframverbindungen sind nur marginale Unterschiede zu verzeichnen. So sind z.B. die Bindungen in den Wolframkomplexen gegenüber den Molybdänkomplexen wie auch in den Mono-Oxo-M(IV)Verbindungen beobachtet, verkürzt, allerdings nur geringfügig (etwa $0.02 \AA$ ).

Die Bindungen der direkt das Metall umgebenden Atome sind deutlich länger als in den Monooxoverbindungen, vor allem die Metall-Schwefel-Bindungen. Die MetallSauerstoffbindungen sind bei beiden Sauerstoffatomen gleichlang und mit durchschnittlich $1.74 \AA$ nur wenig länger als im Edukt. Der von den beiden Sauerstoffatomen 
aufgespannte Winkel liegt etwas über $100^{\circ}$ und ist deutlich größer als er in einem regulären Oktaeder betragen sollte.

Die Metall-Schwefel-Bindungen unterscheiden sich derart, dass sie an dem zu einem Liganden gehörenden Schwefel-Atomen unterschiedlich lang sind $(2.7505 \AA$ und $2.5053 \AA \mathrm{im}\left[\mathrm{Mo}^{V I} \mathrm{O}_{2}(\mathrm{pdt})_{2}\right]^{2-}$ und zwar bei beiden Liganden gleichermaßen. So betragen die Abstände im Durchschnitt für die beiden in der äquatorialen Ebene liegenden Schwefel-Atome eines Liganden M(1)-S(2) und M(1)-S(3) $2.75 \AA$ und $2.50 \AA$. Die Abstände M(1)-S(4) und M(1)-S(5) betragen wiederum im Mittel $2.75 \AA$ und $2.5 \AA$ obwohl das Atom S(4) eine axiale Position einnimmt, während das Atom S(5) in der äquatorialen Ebene liegt. Die C-C-Doppelbindungen weisen mit durchschnittlich 1.34 A keine großen Veränderungen zur Ausgangsverbindung auf. Auch die S-CBindungslängen liegen mit $1.74 \AA$ bis $1.76 \AA$ im dem Bereich der Monooxoverbindungen.

Der Diederwinkel S(2)-S(3)-O(eq)-S(5) zeigt mit einer Größe von 6-7 eine geringe Verzerrung innerhalb der äquatorialen Ebene. Die Verzerrung des Oktaeders wird auch deutlich am Winkel der sich gegenüberstehenden axialen Liganden, welcher durchschnittlich $160^{\circ}$ beträgt und um $20^{\circ}$ von der idealen Oktaederform $\left(180^{\circ}\right)$ abweicht.

Die ab initio-Energie der Ausgangsverbindungen und Produkte sowie die berechneten Energien für den Sauerstofftransfer von DMSO zu DMS sind in Tabelle 2.35 dargestellt.

Es zeigt sich deutlich, dass die Größenordnungen selbst bei einem Wechsel des Liganden gleich bleiben, dass jedoch bei einem Wechsel des Metalls enorme Unterschiede auftreten. So liegen die Energiengewinne bei den Molybdänverbindungen zwischen 30 und $40 \mathrm{~kJ} / \mathrm{mol}$ während sich bei den Wolframverbindungen Werte von etwa 130 $\mathrm{kJ} / \mathrm{mol}$ ergeben. Dieser enorme Unterschied zwischen den beiden Metallen wurden 
Tabelle 2.35: Energien Oxoaffinität der verschiedenen Systeme

\begin{tabular}{lcllc}
\hline & $\mathrm{E}_{\text {Ausgangsverbindung }}[\mathrm{H}]^{a}$ & $\mathrm{E}_{\text {Produkt }}[\mathrm{H}]$ & Exergonie $[\mathrm{kJ} / \mathrm{mol}]$ \\
\hline$\left[\mathrm{Mo}^{I V} \mathrm{O}(\mathrm{pyr})_{2}\right]^{2-}$ & -2274.67629 & {$\left[\mathrm{Mo}^{V I} \mathrm{O}_{2}(\mathrm{pyr})_{2}\right]^{2-}$} & -2349.88724 & -35.87 \\
{$\left[\mathrm{~W}^{I V} \mathrm{O}(\mathrm{pyr})_{2}\right]^{2-}$} & -2274.97945 & {$\left[\mathrm{~W}^{V I} \mathrm{O}_{2}(\mathrm{pyr})_{2}\right]^{2-}$} & -2350.22664 & -131.00 \\
{$\left[\mathrm{Mo}^{I V} \mathrm{O}(\mathrm{chr})_{2}\right]^{2-}$} & -2579.64139 & {$\left[\mathrm{Mo}^{V I} \mathrm{O}_{2}(\mathrm{chr})_{2}\right]^{2-}$} & -2654.85391 & -39.97 \\
{$\left[\mathrm{~W}^{I V} \mathrm{O}(\mathrm{chr})_{2}\right]^{2-}$} & -2579.94432 & {$\left[\mathrm{~W}^{V I} \mathrm{O}_{2}(\mathrm{chr})_{2}\right]^{2-}$} & -2655.19327 & -135.64 \\
{$\left[\mathrm{Mo}^{I V} \mathrm{O}(\mathrm{prz})_{2}\right]^{2-}$} & -2648.57037 & {$\left[\mathrm{Mo}^{V I} \mathrm{O}_{2}(\mathrm{prz})_{2}\right]^{2-}$} & -2723.78010 & -32.64 \\
{$\left[\mathrm{~W}^{I V} \mathrm{O}(\mathrm{prz})_{2}\right]^{2-}$} & -2648.87322 & {$\left[\mathrm{~W}^{V I} \mathrm{O}_{2}(\mathrm{prz})_{2}\right]^{2-}$} & -2724.11923 & -127.92 \\
{$\left[\mathrm{Mo}^{I V} \mathrm{O}(\mathrm{mpt})_{2}\right]^{2-}$} & -3281.44964 & {$\left[\mathrm{Mo}^{V I} \mathrm{O}_{2}(\mathrm{mpt})_{2}\right]^{2-}$} & -3356.66089 & -36.66 \\
{$\left[\mathrm{~W}^{I V} \mathrm{O}(\mathrm{mpt})_{2}\right]^{2-}$} & -3281.75244 & {$\left[\mathrm{~W}^{V I} \mathrm{O}_{2}(\mathrm{mpt})_{2}\right]^{2-}$} & -3356.99971 & -131.22 \\
DMSO & -553.26018 & & & \\
DMS & -478.06289 & & &
\end{tabular}

${ }^{a} 1$ Hartree $=2625.4999638 \mathrm{~kJ} / \mathrm{mol}$

auch von Garner et al. beobachtet [132]. An deren Modellen wurden für die Molybdänverbindungen Werte zwischen 80 und $90 \mathrm{~kJ} / \mathrm{mol}$ erhalten während die Wolframverbindungen mit Werten von 160 bis $180 \mathrm{~kJ} / \mathrm{mol}$ deutlich darüber liegen 16 .

Das Profil der Oxo-Transfer-Reaktion war Gegenstand zahlreicher theoretischer Untersuchungen [133, 134]. Vor allem die Arbeiten von Webster und Hall trugen sehr zum Verständnis bei und führten zu einem vorgeschlagenen zweistufigen Mechanismus der Sauerstoffübertragung.

Garner et al. veröffentlichten im Jahr 2005 eine umfangreiche Studie, deren Gegenstand der Vergleich beider Metalle koordiniert durch eines dem Molybdopterin bis auf den fehlenden Pyranring ähnlichen Ligandensystems war 132]. Diese Untersuchung zeigte, dass sich die Reaktionsprofile deutlich unterscheiden. Im Falle der Molybdänverbindung liegt der erste Überganszustand energetisch tiefer als der zweite, wohingegen bei der Wolframverbindung beide Sattelpunkte nahezu gleich hoch liegen. Ein signifikanter Unterschied besteht hinsichtlich der Aktivierungsbarrierenhöhe, welche bei den Wolframverbindungen gegenüber den Molybdänverbindungen um $23 \mathrm{~kJ} / \mathrm{mol}$

\footnotetext{
${ }^{16}$ Unterschiede begründet die Natur des anderen Liganden sowie die verwendeten Methoden und Basissätze
} 
Tabelle 2.36: Energien des Sauerstofftransfers

\begin{tabular}{lc}
\hline Verbindung & $\mathrm{E}_{\text {abinitio }}[\mathrm{H}]$ \\
\hline$\left[\mathrm{Mo}^{I V} \mathrm{OCH}_{3}(\mathrm{edt})\left(\mathrm{mpt}_{\text {offen }}\right)\right]^{1-}$ & -2625.95158 \\
{$\left[\mathrm{Mo}^{V I} \mathrm{O}_{2} \mathrm{CH}_{3}(\mathrm{edt})\left(\mathrm{mpt}_{\text {offen }}\right)\right]^{1-}$} & -2701.17308 \\
{$\left[\mathrm{Mo}^{I V} \mathrm{OCH}_{3}(\mathrm{edt})\left(\mathrm{mpt}_{\text {geschl. }}\right)\right]^{1-}$} & -2625.97187 \\
{$\left[\mathrm{Mo}^{V I} \mathrm{O}_{2} \mathrm{CH}_{3}(\mathrm{edt})\left(\mathrm{mpt}_{\text {geschl. }}\right)\right]^{1-}$} & -2701.19304 \\
{$\left[\mathrm{Mo}^{V} \mathrm{OCH}_{3}(\mathrm{DMSO})(\mathrm{edt})\left(\mathrm{mpt}_{\text {offen }}\right)\right]^{1-}$} & -3179.05476 \\
{$\left[\mathrm{Mo}^{V} \mathrm{OCH}_{3}(\mathrm{DMSO})(\text { edt })\left(\mathrm{mpt}_{\text {geschl. }}\right)\right]^{1-}$} & -3179.07410 \\
& \\
DMSO & -553.13127 \\
DMS & -477.94367
\end{tabular}

\begin{tabular}{lcccc}
\hline & $\mathrm{E}_{\text {Ausgangsverb. }}[\mathrm{kJ} / \mathrm{mol}]$ & $\mathrm{E}_{T S}[\mathrm{~kJ} / \mathrm{mol}]$ & $\mathrm{E}_{\text {Produkt }}[\mathrm{kJ} / \mathrm{mol}]$ & $\nu\left[\mathrm{cm}^{-1}\right]^{a}$ \\
\hline geschlossene Form & 0 & 76.24 & -88.13 & -295 \\
offene Form & 0 & 73.75 & -88.99 & -289 \\
${ }^{a}$ imaginäre Frequenz der Reaktionskoordinate & & &
\end{tabular}

tiefer liegt.

Gänzlich unbeachtet blieb jedoch bisher die Betrachtung des Einflusses einer Ringöffnung bei Systemen welche über die üblichen Modellverbindungen hinausgehen.

Wie bereits in Kap. 2.4 ausführlich dargelegt, bieten die hier untersuchten Modellsysteme Gelegenheit, die Reaktion der Ringöffnung näher zu untersuchen. Als Erweiterung der energetischen Betrachtung der offenen und geschlossenen Form wurden erste explorative Untersuchungen 17 der Sauerstoffübertragungsreaktion bezüglich einer möglichen Ringöffnung während des Reaktionsverlaufs durchgeführt. Der hier verwendete Modellkomplex verfügt aufgrund des geringeren Rechenaufwandes im Gegensatz zu den bisher behandelten Modellen über nur einen Molybdopterinliganden, wobei der zweite Ligand lediglich die Dithioleneinheit $\left(\mathrm{edt}^{2-}\right)$ beinhaltet. Des weiteren ist am axialen Sauerstoff-Atom eine Methylgruppe gebunden, die die hohe Ladung abschirmt und so die Bestimmung des Übergangszustandes erleichtert.

Die erhaltenen Energien sowie die Energiedifferenzen der Ausgangsprodukte, des

\footnotetext{
${ }^{17}$ Für die Untersuchung der Sattelpunkte fand das Programmpaket Turbomole Anwendung.
} 
Abbildung 2.23: Reaktionsprofile der Sauerstoffübertragung
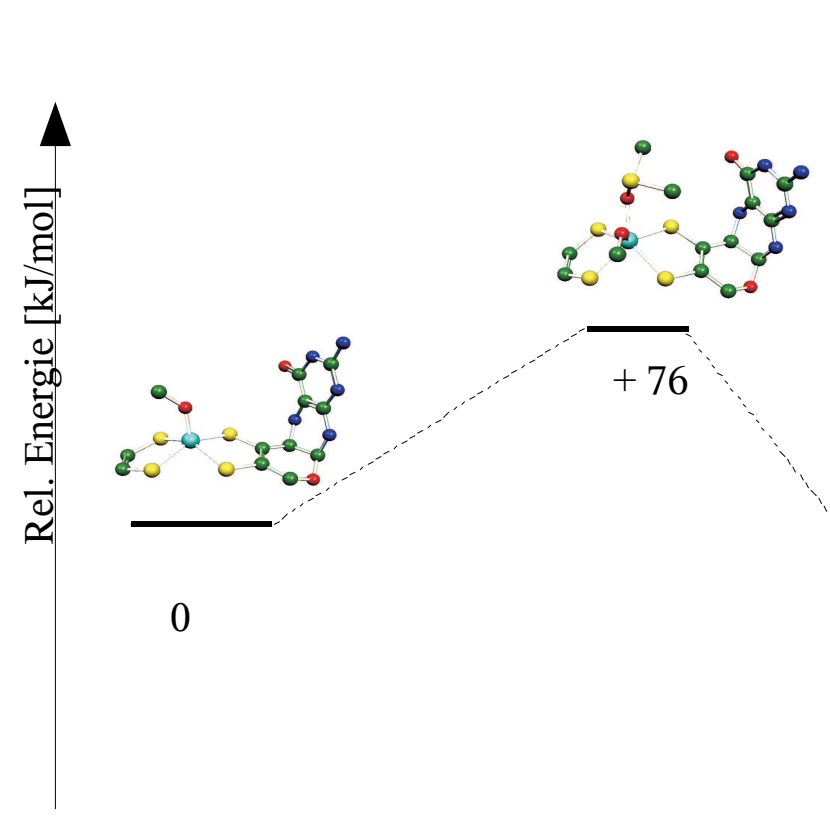

$+76$
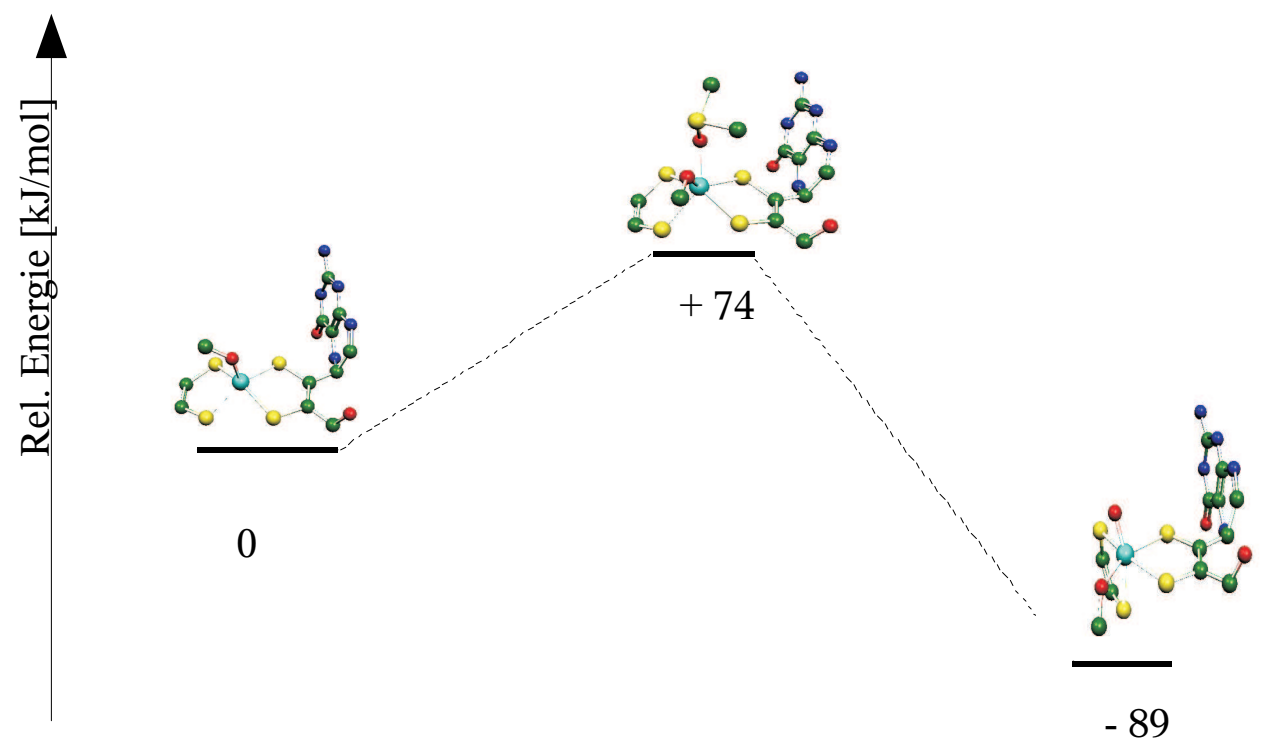
Übergangszustands und der Produkte sind in Tabelle 2.36 dargestellt. Auffallend hierbei ist die hohe Übereinstimmung der Aktivierungsenergie sowie der Exergonie bei beiden Tautomeren. Die Werte liegen bei 76.24 bzw. $73.25 \mathrm{~kJ} / \mathrm{mol}$ (geschlossene Form bzw. offene Form) für den TS und bei -88.13 bzw. $-88.99 \mathrm{~kJ} / \mathrm{mol}$ (geschlossene Form bzw. offene Form) für die Exergonie. Diese Werte des Übergangszustands zeigen eine gute quantitative Übereinstimmung mit den von Garner et al. berechneten Werten für die Modelle der offenen Form.

Besonders hervorzuheben ist hierbei jedoch, dass das Reaktionsprofil der offenen Formen insgesamt um $50 \mathrm{~kJ} / \mathrm{mol}$ höher liegt als das der geschlossenen Homologen 18 . Die Extrempunkte der Reaktionskurve (Abbildung 2.23) beider Tautomeren weisen dieselben Energiedifferenzen auf und damit ergibt sich in direkter Folge, dass die Reaktion über beide Formen gleichermaßen möglich ist.

Der Energieunterschied zu Gunsten der geschlossenen Form ist in Übereinstimmung mit den in Kapitel 2.4 dargelegten Werten. Wie dort besprochen, kann auch hier die nötige Stabilisierung durch die Umgebung des Proteins z.B. über H-Brücken aus der Enzymumgebung durchaus aufgebracht werden, so dass eine Ringöffnung grundsätzlich möglich ist.

\subsection{Diskussion aller Systeme im Vergleich}

Um einen allgemeinen Überblick zu bekommen, sind hier die wichtigsten Eigenschaften der verschiedenen Systeme gegenübergestellt.

In jedem Fall konnte die Tendenz erkannt werden, dass bei Zunahme der OS, also dem Entzug zweier Elektronen, die das Metall umgebenen Atome eine Bindungsverkürzung (M-O, M-S und C-S) erfuhren. Bis auf die C-C-Doppelbindung, die leicht länger wird, setzt sich diese Tendenz im gesamten Molekül fort, wobei die Auswirkungen

\footnotetext{
${ }^{18}+53.26 \mathrm{~kJ} / \mathrm{mol}$ Ausgangsverbindung $,+50.77 \mathrm{~kJ} / \mathrm{mol} \mathrm{TS},+52.4 \mathrm{~kJ} / \mathrm{mol}$ Produkt
} 
mit zunehmendem Abstand zum Metall schwächer werden. Die Zunahme des kovalenten Charakters, welcher sich in der Bindungsverkürzung äußert, resultiert aus der Umverteilung der beweglichen $\pi$-Elektronendichte der benachbarten C-C-Doppelbindung. Als direkte Folge der abnehmenden $\pi$-Elektronendichte dort verlängern sich die C-CAbstände signifikant.

Die Metall-Schwefel-Bindungen sind in den Wolframkomplexen immer kürzer als bei den Molybdänkomplexen, wenn auch ihre jeweiligen Tendenzen hinsichtlich der Veränderung bei steigender Oxidationszahl, ähnliche Größenordnungen aufweisen. Die Metall-Sauerstoff-Bindung ist beim Wolfram nur leicht kürzer als beim Molybdän. Hier zeigt sich deutlich die stärkere KLZ sowie die Auswirkung der relativistischen Effekte, wodurch eine stärkere Kontraktion der Elektronenhülle, resultierend in der stärkeren Anziehung der das Wolfram umgebenden Atome zu sehen ist. Das partielle Ausbilden von Metall-Schwefel-Doppelbindungen konnte bei allen Systemen in der OS VI beobachtet werden.

Dieses Phänomen wird durch Interaktion des Metalls mit den redoxaktiven Dithioleneinheiten möglich, so dass die Schwefelatome in der Situation des Elektronenmangels am Metall ein freies Elektronenpaar zur Ausbildung der zweiten Bindung zur Verfügung stellen können. Möglich ist das durch das elektronenreiche Rückgrat, vor allem die Verfügung über die locker gebundenen $\pi$-Elektronen der C-C-Doppelbindung die wiederum durch Konjugation mit dem angrenzenden Ring leicht Elektronen weitergeben kann. Am Beispiel der Pyranylkomplexe ist hier wieder die stärkere Neigung Wolframs zu Doppelbindungen mit Schwefel zu erkennen, dessen Komplex in OS VI zwei solcher Bindungen ausbildet. Auch der Sonderfall bei den aromatischen Verbindungen, bei denen es zu einer destabilisierenden Wechselwirkung aufgrund eines zu starken Elektronenflusses aus dem aromatischen Rückgrat kommt, wird durch die hohe Eletronendichte-Anziehungskraft des Wolframs abgefangen. 
Die Wechselwirkung mit den an den Pyranring grenzenden Systemen (Benzolring bzw. Pyrazinring) ist mit der Verteilung der einzelnen freien Elektronenpaare bzw. ihrer Beimischungen anhand der Bindungsanalyse nachgewiesen worden. Ein auffälliges Verhalten zeigen die aromatischen Systeme, bei denen es durch zu starke Konjugation mit dem aromatischen Rückgrat sogar zu einer antibindenen Wechselwirkung kommt.

Die bei den Pyrazin- und Molybdopterinverbindungen abgeschwächte Konjugation ist dennoch anhand der unterschiedlichen M-S-Bindungslängen festzustellen.

Die p-Orbitale der Schwefelatome haben in den sich gegenüberliegenden Liganden unterschiedliche Phasen. Die durch Konjugation verteilte Elektronendichte stößt bei dem sich auf der jeweils anderen Seite befindenden Liganden auf ein antibindendes p-Orbital, so dass die jeweils betroffene Bindung leicht verlängert wird. Das zeigt sich auch an den Größenordnungen der in trans-Form unterschiedlich langen Bindungen. Während die Pyranylverbindungen, welche keinerlei Möglichkeit haben, Elektronen aus dem Rückgrat zu involvieren, gleichmäßige M-S-Abstände aufweisen, sind diese Unterschiede nur in den größeren Systemen vorhanden. Die aromatischen Systeme, welche die stärkste Konjugation aufweisen, zeigen auch die größten Diefferenzen der M-S-Bindung zwischen den benachbarten Schwefel-Atomen, während die Unterschiede sowohl bei den Pyrazin- als auch bei den Molybdopterinverbindungen eher moderat ausfallen.

Die NPA-Ladungen der Schwefel-Atome sind an den länger gebundenen SchwefelAtomen weniger stark als an den kürzer gebundenen, während im Pyranylsystem alle Schwefel-Atome über den gleichen Ladungsanteil verfügen.

In den Systemen Pyrazin und Molybdopterin ist die $\pi$-Elektronendichte aus dem Pteridingerüst zwar vorhanden und aufgrund ihrer Konformation zur Konjugation befähigt, aber aufgrund der hohen Elektronegativität und dem damit verbunden -IEffekt stärker an den Stickstoff-Atomen lokalisiert. Das Pteridin agiert in bezug auf 
das elektronische Verhalten ähnlich einem stark desaktivierten Aromaten, der nur über eine abgeschwächte Mesomerie verfügt.

Die sich in umgekehrter Phase gegenüberstehenden p-Orbitale des Schwefels sind auch mit für das Phänomen des Faltungswinkels verantwortlich. Wie oben diskutiert, vergrößert das Metall durch die Faltung die Überlappung mit dem Schwefel auf einer Seite, während es bei dem Liganden auf der anderen Seite zu einer Abstoßung führt, hervorgerufen durch die in unterschiedlicher Phase zueinander stehenden p-Orbitale.

Innerhalb der verschiedenen Ligandensysteme zeigen sich weitere Trends. Abbildung 2.24 zeigt die Mo-S-Bindungen (Trend bei Wolfram analog) sowie die C-CDoppelbindungen aller Systeme im Vergleich bei Durchlaufen der Oxidationsschritte sowie den Vergleich der C-C-Doppelbindung mit der analogen Bindung in den unsubstituerten Dithiolenkomplexen $\left[\mathrm{MO}(\text { edt })_{2}\right]^{z-}(\mathrm{M}=\mathrm{Mo}, \mathrm{W} ; \mathrm{z}=-2,-1,0)$.

Auffallend bei den Veränderungen der Mo-S-Bindungslängen ist das Abflachen der Abnahme mit zunehmender Annäherung des Liganden an das Molybdopterin und bei den Molybdopterinverbindungen selbst. Während die Abnahme der Bindungslänge bei den Pyranylkomplexen nahezu monoton verläuft, ist die Verkürzung bei den Chromanyl- und Flavanylverbindungen schon weniger stark und bei den Pyrazin- und Molybdopterinverbindungen nochmals geringer. Eine Ausnahme bilden die Flavanylverbindungen, welche von vornherein kürzere Abstände als die Pyranylverbindungen aufweisen. Die unsubsituierten Dithiolenkomplexe zeigen jedoch die größte Abweichung mit einer viel längeren Mo-S-Bindung in den OS IV und V, während sie sich in OS VI den anderen Systemen annähern.

Die Änderungen in der C-C-Doppelbindungslänge weisen ähnliche Besonderheiten auf. Die Abstände vergrößern sich und zwar ist die Änderung bei den Pyrazin- und Molybdopterinsystemen wieder am geringsten. Diesmal sind die Pyranylkomplexe den beiden Systemen näher und die größten Änderungen sind in den Systemen Chromanyl 
Abbildung 2.24: Tendenzen der Bindungen Me-S und C-C

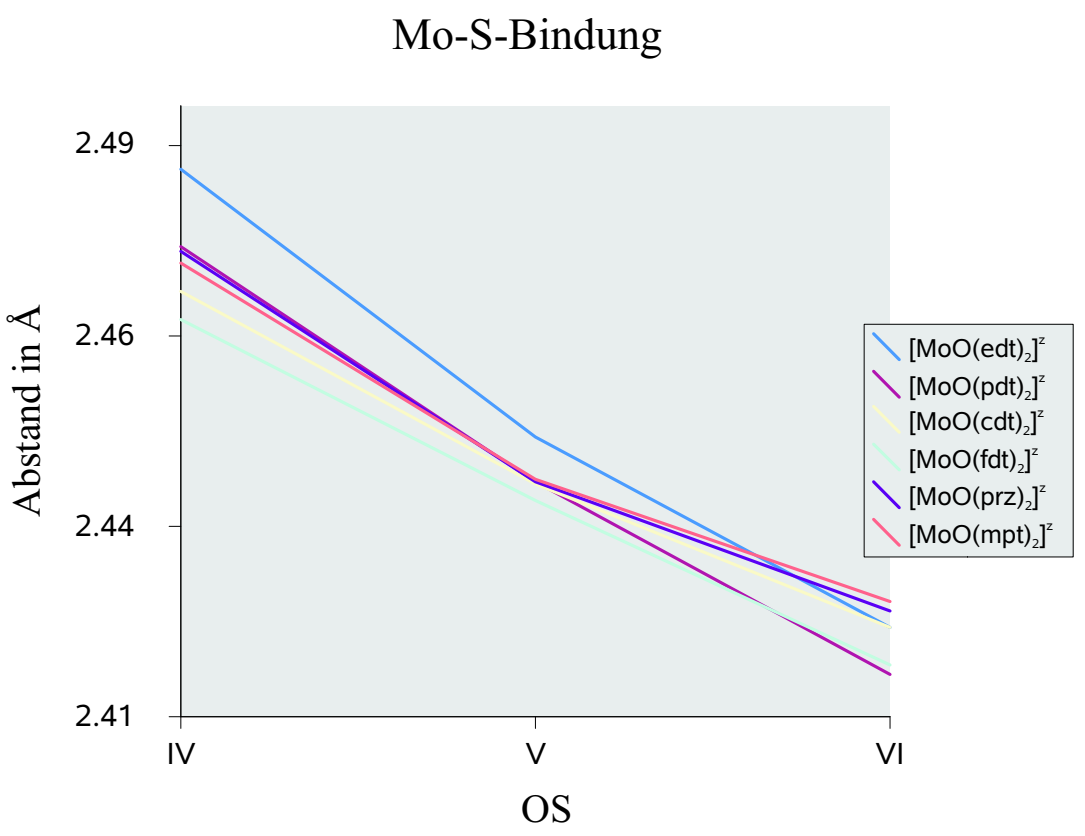

C-C-Doppelbindung

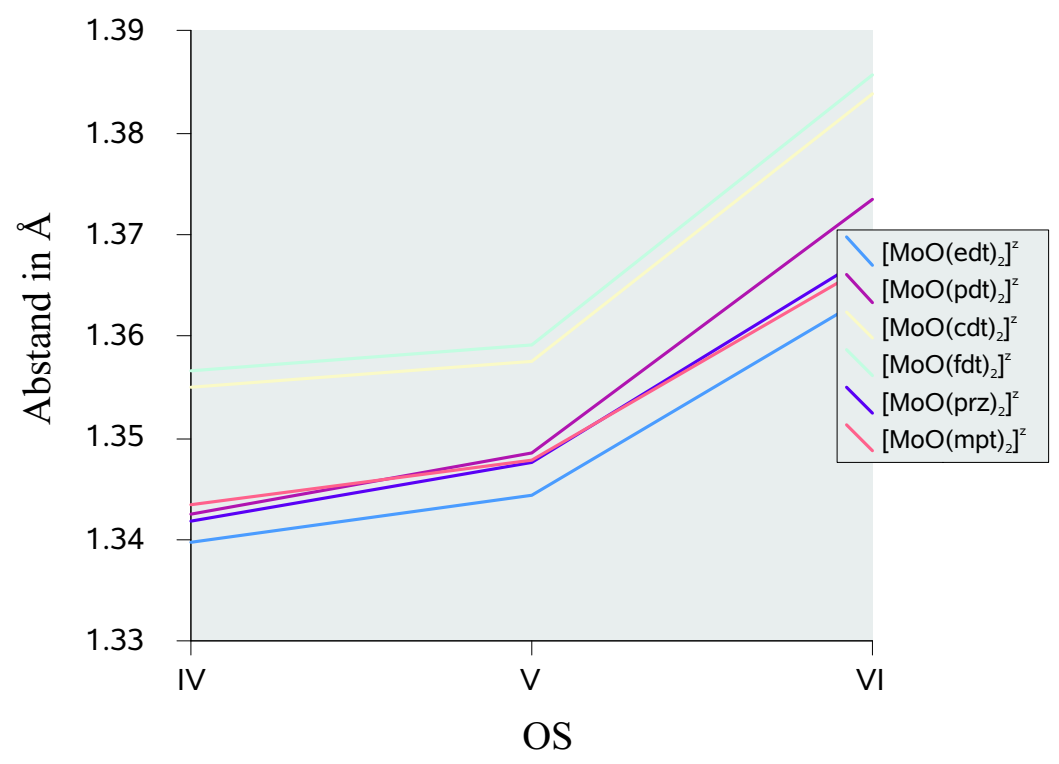


und Flavanyl zu beobachten. Da die Bindungslänge im Vergleich zu einer reinen CC-Doppelbindung Aufschluss über das Maß an Delokalisierung bietet, zeigt sich hier, dass die Delokalisierung im Vergleich dazu weitaus geringer ist, bei den aromatischen Systemen aber bedingt durch das höhere Ausmaß an Konjugations noch am höchsten ist. Die im Vergleich dazu recht schwachen Veränderungen bei den Pyranyl-, Pyrazinund Molybdopterinsystemen beruhen auf der beim Pyrazin und Molybdopterin eingeschränkten Möglichkeit der Weitergabe von Elektronen und im Pyranyl auf dem Fehlen eines Rückgrates überhaupt. Die wesentlich kürzeren Bindungslängen in den unsubstituierten Dithiolenkomplexen zeigen deutlich, dass das bei den anderen Systemen zusätzlich vorhandene Ligandengerüst einen großen Einfluss auf das Verhalten der Dithioleneinheit hat.

Eine weitere geometrische Besonderheit stellt das Auftreten der unterschiedlichen Faltung beider Liganden dar. Wie oben erläutert, knickt je ein Ligand bei Erreichen der OS V und VI nach oben ab um den Elektronenmangel am Metall durch die so mögliche bessere Überlappung mit dem Schwefel zu erreichen. Der Trend ist bei allen Systemen ähnlich mit einem starken Anstieg beim Übergang von OS IV zu V und einem etwas flacheren Anstieg beim Übergang von OS V zu VI.

Wieder ist zu beobachten, dass die Änderungen bei den Pyrazin- und Molybdopterinverbindungen am geringsten, bei den Flavanyl- und Pyranylverbindungen am stärksten sind und bei den Chromanylverbindungen zwischen den beiden Extremen liegt. Unterschiede zwischen den beiden Metallen sind bis auf Abweichungen von wenigen Grad zugunsten des Wolframs nicht zu beobachten. Das unsubstituierte Dithiolen dagegen wird beim Wechsel von OS IV zu OS V weniger stark und beim Wechsel von OS V zu VI mehr gefaltet als die anderen Systeme.

Aber auch der flach verbleibende gegenüberliegende Ligand unterliegt einigen Veränderungen, dargestellt in Diagramm 2.26 . 
Abbildung 2.25: Faltungswinkel aller Systeme

Faltungswinkel Mo-[S(4)-S(5)]

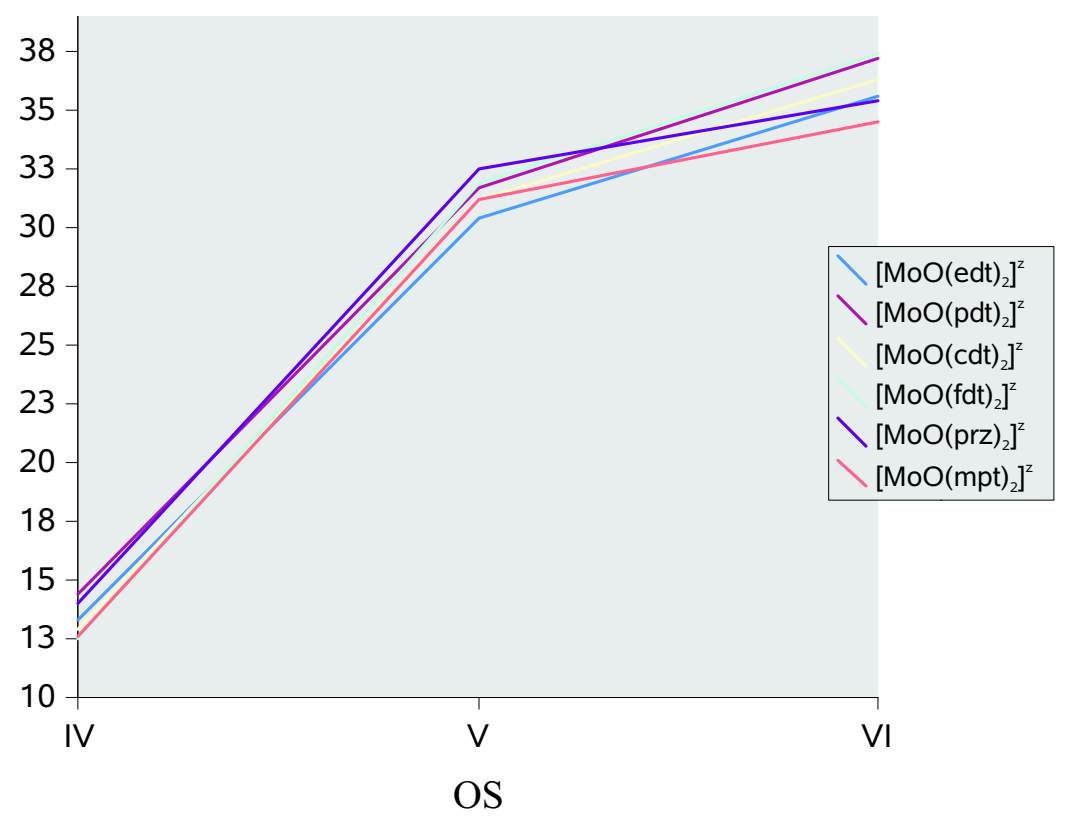

Abbildung 2.26: Faltungswinkel aller Systeme

Faltungswinkel M-[S(2)-S(3)], M = Mo, W

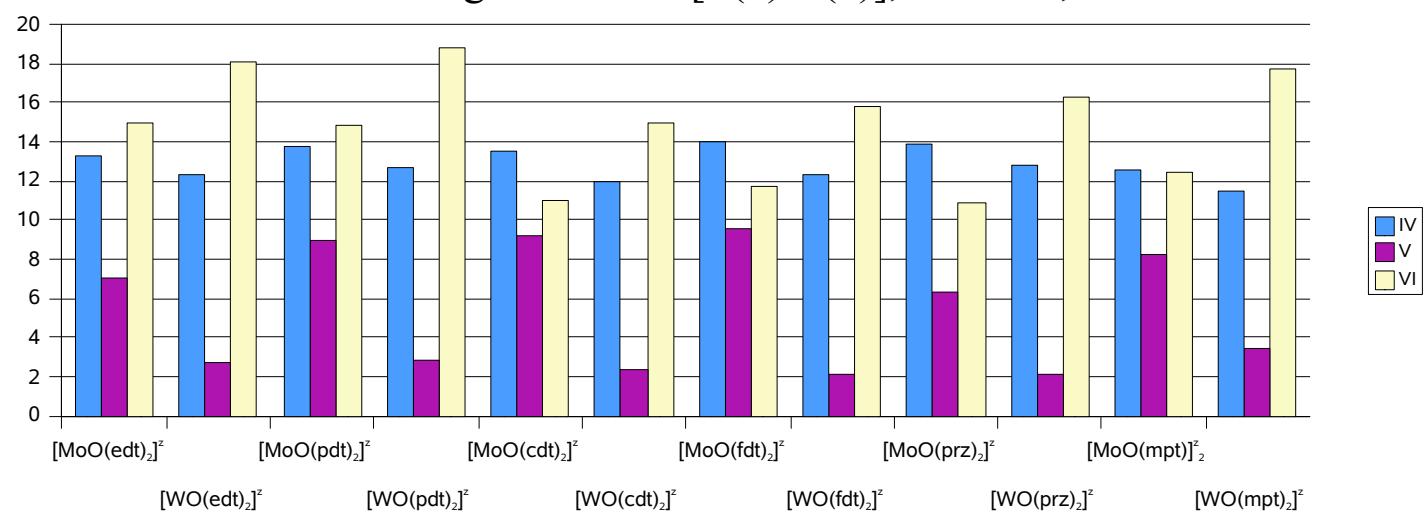


In der symmetrisch 19 vorliegenden Form in OS IV betragen die Faltungswinkel auf beiden Seiten etwa $12-14^{\circ}$. In Oxidationstufe V sinkt der nicht von der Faltung betroffene Ligand beim Molybdän auf 7-8 ab, beim Wolfram sogar bis auf $2-3^{\circ}$. In OS VI dagegen steigt dieser Winkel wieder an, beim Molybdän nahe an den ursprünglichen Wert mit Beiträgen zwischen 11 und $14^{\circ}$ und beim Wolfram sogar bis auf $18^{\circ}$. Scheinbar ist es dank der starken Kernladungszahl des Wolfram hier möglich zusätzlich die Überlappung durch Faltung zu stärken ohne dass abstoßende Wechselwirkungen mit dem gegenüberliegenden Liganden eintreten, da Wolfram die Elektronendichte stark an sich zieht.

Eine weitere Gemeinsamkeit die in allen Systemen beobachtet wird, ist die Verkürzung der Abstände S(2)-S(3) bzw. S(4)-S(5). Die in OS IV auf beiden Seiten gleichlangen Abstände verkürzen sich bei Oxidation auf unterschiedliche Weise. Bei den Molybdänkomplexen ist eine stärkere Verkürzung der Bindungslänge S(2)-S(3) (im Mittel (Molybdopterinverb.) von 3.26 auf $3.20 \AA$ ) zu sehen als bei der Bindungslänge S(4)-S(5) (von 3.26 auf $3.24 \AA$ ) während sich bei den Wolframkomplexen nur die Länge S(2)-S(3) verkürzt (von 3.23 auf $3.19 \AA$ ). In Hinblick auf die von Inscore und Naughton [125, 126] postulierte oxo-gate-Hypothese scheinen die Schwefel-Atome in der Elektronenmangelsituation in OS VI zumindest auf der nicht gefalteten Seite einander anzunähern um weitere Elektronendichte übertragen zu können.

Beobachtet werden konnte das Verkürzen dieser Bindung während der Oxidation auch in der Aldehyoxidoreduktase von D. gigas [135]. Hier vergrößerte sich der Abstand der beiden Schwefel-Atome von 3.0 auf 3.5 $\AA$ wobei der Winkel S-Mo-S ebenfalls zunahm. Die dadurch erhöhte Kovalenz zwischen den beiden Atomen erhöht so die verfügbare Elektronendichte und kann dadurch auch das Redoxpotential beeinflussen bzw. hohe OS am Molybdän stabilisieren.

\footnotetext{
${ }^{19}$ abgesehen von der trans-Stellung der Liganden nahezu $c_{2} v$-Symmetrie
} 
Tabelle 2.37: HOMO-LUMO-Gap [a.u.]

\begin{tabular}{lccc}
\hline & IV & V & VI \\
\hline$\left[\mathrm{MoO}(\mathrm{pdt})_{2}\right]^{z-}$ & 0.15 & 0.13 & 0.08 \\
{$\left[\mathrm{WO}(\mathrm{pdt})_{2}\right]^{z-}$} & 0.14 & 0.15 & 0.08 \\
{$\left[\mathrm{MoO}(\mathrm{cdt})_{2}\right]^{z-}$} & 0.11 & 0.12 & 0.08 \\
{$\left[\mathrm{WO}(\mathrm{cdt})_{2}\right]^{z-}$} & 0.10 & 0.13 & 0.08 \\
{$\left[\mathrm{MoO}(\mathrm{fdt})_{2}\right]^{z-}$} & 0.10 & 0.12 & 0.08 \\
{$\left[\mathrm{WO}(\mathrm{fdt})_{2}\right]^{z-}$} & 0.09 & 0.13 & 0.08 \\
{$\left[\mathrm{MoO}(\mathrm{prz})_{2}\right]^{z-}$} & 0.14 & 0.13 & 0.08 \\
{$\left[\mathrm{WO}(\mathrm{prz})_{2}\right]^{z-}$} & 0.13 & 0.15 & 0.09 \\
{$\left[\mathrm{MoO}(\mathrm{mpt})_{2}\right]^{z-}$} & 0.08 & 0.13 & 0.09 \\
{$\left[\mathrm{WO}(\mathrm{mpt})_{2}\right]^{z-}$} & 0.06 & 0.14 & 0.09
\end{tabular}

Damit im Einklang ist auch der Gang des Winkels des axialen Sauerstoffs bezüglich der S-S-Ebene. Während er in OS IV auf beiden Seiten $114^{\circ}$ (Bsp. Molybdopterinverb.) beträgt, nimmt er auf der gefalteten Seite mit zunehmender Oxidation leicht ab $\left(112 / 110^{\circ}, \mathrm{Mo} / \mathrm{W}\right)$ und neigt sich in Richtung des gefalteten Liganden. Nach Inscore und Naughton [125, 126] ist die Wechselwirkung der in-plane-p-Orbitale der SchwefelAtome mit den $\mathrm{d}_{x^{2} y^{2}}$-Orbitalen des Metalls umso größer, je orthogonaler der Sauerstoff gegenüber dem Liganden steht. So vergrößert sich die Überlappung auf der Seite des gefalteten Liganden während sich dieser Winkel auf der anderen Seite noch vergrößert $\left(123^{\circ} \mathrm{Mo}, 122^{\circ} \mathrm{W}\right)$ und hier die Überlappung verringert wird. Die Verkürzung des $\mathrm{S}(2)-\mathrm{S}(3)$-Abstands geht also mit der wachsenden Entfernung des axialen Sauerstoffs einher.

Die HOMO-LUMO-Abstände der Komplexe in den OS IV, V und VI sind in Tabelle 2.37 dargestellt.

Typisch für geladene Komplexe sind die Orbitalenergien in den positiven Bereich angehoben. Der Abstand des höchsten besetzten zum ersten virtuellen Orbital liegt im Bereich zwischen 0.06 a.u. $\left(\left[\mathrm{WO}(\mathrm{mpt})_{2}\right]^{2-}\right)$ bis 0.15 a.u. $\left(\left[\mathrm{MoO}(\mathrm{pdt})_{2}\right]^{2-}\right)$. Bei allen Systemen ist die Lücke für die Wolframkomplexe um 0.01 a.u. bis 0.02 a.u. kleiner. Die 
Werte sind für den Pyranylkomplex deutlich am höchsten. Die aromatischen Systeme schneiden beide recht ähnlich ab und liegen deutlich unter den Pyranylverbindungen. Die Pyrazinverbindungen wiederum liegen interessanterweise recht nah an den Werten für die Pyranylkomplexe wogegen bei den Molybdopterinverbindungen die Werte mit 0.08 a.u. für Molybdän und 0.06 a.u. für Wolfram am tiefsten liegen.

Das Verkleinern der Lücke bei den Molybdopterinverbindungen zeigt die deutliche Zunahme der Reaktivität bzw. der Oxidierbarkeit, in dem Sinne, dass die unbesetzten Orbitale während einer möglichen Reaktion für die Ausbildung eines aktivierten Komplexes leichter zugänglich sind. Demnach sollte ein Angriff auf einen der Molybdopterinkomplexe, verglichen mit den anderen Systemen, am leichtesten möglich sein.

Die Werte der Orbitalkoeffizienten zeigen eine deutliche Tendenz sowohl bei Molybdän als auch bei Wolfram und zwar bei allen Systemen. Beim Wechsel von OS IV zu V ist ein Absinken des metallischen Charakters unter Zunahme des p-Anteils der Schwefel-Atome zu beobachten, während in OS VI wiederum ein Anstieg des metallischen Charakters erfolgt, welcher aber unter dem Wert der elektronischen Dichte in OS IV bleibt. Eine Ausnahme bildet der Molybdän-Chromanylkomplex in OS IV, dessen HOMO aufgrund einer Orbitalinversion mit dem normalerweise tiefer liegenden Schwefel-p-Orbital vertauscht ist und daher nicht mit den anderen Systemen vergleichbar ist.

Die Abnahme des Metallcharakters in OS V wird durch das Entfernen eines Elektrons bewirkt, so dass die elektronische Dichte am Metall vorerst absinkt. In OS VI kann die elektronische Dichte am Metall wieder ansteigen, da sich das Molekül über den Faltungswinkel stabilisiert. Aufgrund der zusätzlichen Überlappung durch den Faltungswinkel ist es nun möglich Elektronendichte aus dem Dithiolen-Rückgrat über die Schwefel-Atome an das Metall zurückzugeben. 
Bei den Pyranyl- und Flavanylsystemen ist die elektronische Dichte am Wolfram höher. Die Unterschiede sind bei den Pyranylkomplexen mit 40\% Anteil für das Molybdän und $74 \%$ Anteil für das Wolfram viel größer als bei den Flavanylanaloga mit 67\% bzw. 73 \%. Bei den Systemen Pyrazin und Molybdopterin kommt es zu einer weiteren Annäherung der Werte, so dass für beide Metalle nahezu gleiche Verhältnisse beobachtet werden können. Das Molybdän hat mit $73 \%$ gegenüber $70 \%$ (Pyrazinverb., 63/61\% in den Molybdopterinverbindungen) für das Wolfram dabei einen etwas höheren Orbitalkoeffizienten wobei diese Unterschiede nur marginal sind.

Die Analyse der Orbitalkoeffizienten gibt einen ersten Hinweis auf das beobachtete situationsabhängige Einbauverhalten von Molybdän und Wolfram in die MolybdopterinEnzyme. Wie der Vergleich mit dem Pyrazinmodellen zeigt, gelingt es dem Molybdopterin nahezu perfekt, den Metallcharakter von Molybdän und Wolfram anzugleichen. Das hat Einfluss auf den Bindungscharakter beider Metalle, welche sich somit bezüglich ihrer Koordination gleich verhalten. Auch die Ähnlichkeit der Orbitalkoeffizienten in allen OS zeigt, das beide Metalle sich während des katalytischen Prozesses vermutlich identisch verhalten.

Der Vergleich der NPA-Ladungen für alle Systeme zeigt nur geringe Abweichungen beim Verhalten der Schwefel-Atome. Wie oben besprochen, zeigt sich hier der Einfluss der konjugierenden $\pi$-Elektronen aus dem Rückgrat des Liganden. Die Ladungen am Metall und am axialen Sauerstoff sind jedoch bei allen Systemen konstant und folgen einheitlich ihren Trends in den verschiedenen OS. So erfolgt am Metall eine Zunahme der positiven Ladung beim Wechsel in die OS V und eine Abnahme in OS VI, wobei dieser Wert über dem ursprünglichen Wert liegt. Die Positivierung ist beim Wolfram dabei noch stärker. Die Ladung des axialen Sauerstoffs verringert sich mit etwa 0.1 Einheiten wesentlich weniger als die Ladung an den Schwefel-Atomen mit 0.3. Noch geringer fallen die Änderungen mit nur 0.05 Einheiten an der C-C-Doppelbindung 
aus.

Die Verteilung der Ladungen bei dem größten Vertreter, den Molybdopterinverbindungen, zeigt deutlich das sich für beide Metalle in großer Entfernung (ab der C-C-Doppelbindung) vom Metallzentrum die Verhälntnisse fast vollständig entsprechen und nur noch eine marginale Abhängigkeit von der OS besteht. Die Daten aus Diagramm 2.20 für direkt an das Metall gebundendene Atome weisen sowohl für den Sauerstoff als auch den Schwefel eine höhere Partialladung für die Wolframverbindungen auf.

Dieses Ergebnis ist im Einklang mit dem oben beschriebenen Mechanismus des Ladungstransfers welcher die stärksten Änderungen an den redoxaktiven SchwefelAtomen vorhersagt, und so aufzeigt inwieweit sie an dem Redoxprozess beteiligt sind.

Eine entscheidende Größe beim Vergleich der verschiedenen OS ist ihre Energie. Die ab initio-Werte sowie die daraus berechneten Energieunterschiede in $\mathrm{kJ} / \mathrm{mol}$ sind im Diagramm 2.27 dargestellt.

Die Energieunterschiede sind zwischen den OS IV und V geringer als zwischen OS V und VI. OS V ist dabei die stabilste Form. Daher wurden die Unterschiede IV-V und VI -V für die Darstellung verwendet.

Aus Diagramm 2.33 ergibt sich für den Oxidationsprozess von OS V zu OS VI, dass der Ligand nahezu beliebig gewählt werden kann, da die Balkenhöhen in allen Fällen fast deckungsgleich sind.

Ein vollkommen anderes Bild ergibt sich jedoch für den Oxidationsprozess von OS IV zu OS V, da in diesem Fall die Unterschiede zu den Molybdopterinkomplexen augenfällig sind. Während der Energieunterschied bei den Pyranylkomplexen mit 82 bzw. 108 kJ/mol (Mo bzw. W) recht hoch ist, beträgt der Unterschied bei den Pyrazinund Molybdopterinkomplexen nur noch 50/76 kJ/mol (Pyrazinverbindung, Mo/W) bzw. 38/65 kJ/mol (Molybdopterinverbindung, Mo/W). Die aromatischen Systeme 
Abbildung 2.27: Energiedifferenzen

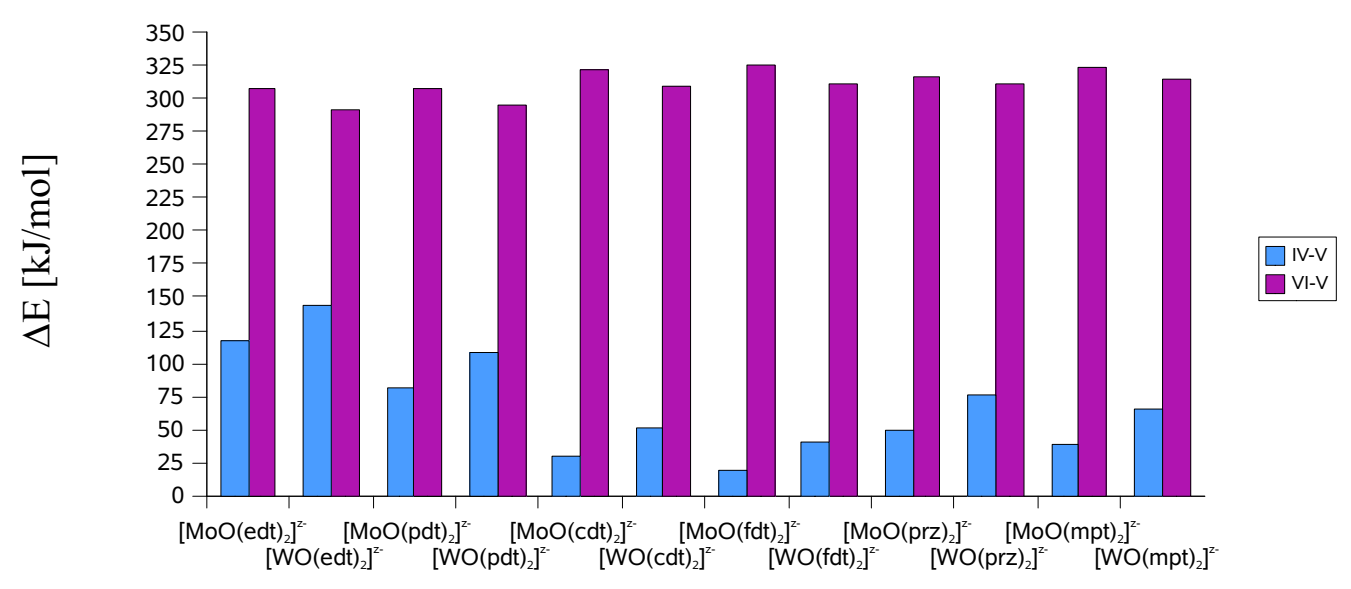

liegen mit 30/52 kJ/mol für die Chromanylverbindung (Mo/W) und $18 / 40 \mathrm{~kJ} / \mathrm{mol}$ für die Flavanylverbindung (Mo/W) sogar unter den Werten für das Molybdopterin, reproduzieren die Situation jedoch besser als die Pyranylverbindungen. Dagegen liegen die Energieunterschiede bei den unsubstituerten Dithiolenkomplexen mit 116/143 $\mathrm{kJ} / \mathrm{mol}(\mathrm{Mo} / \mathrm{W})$ weitaus höher als bei den anderen Systemen. Das zeigt deutlich, dass das Ligandensystem einen entscheidenden Einfluss auf das energetische Verhalten der Verbindungen ausübt, was sich auch auf den katalytischen Prozess auswirkt.

Insgesamt lassen sich folgende allgemeinen Tendenzen ableiten:

Anhand der großen Ähnlichkeiten zwischen den molybdopterinartigen Pyrazinverbindungen, denen lediglich der dritte Ring fehlt und dem Molybdopterinsystem selbst zeigt sich, dass das an den Pyranring geknüpfte Pyrazingerüst das Verhalten am Metallzentrum mit beeinflusst, unabhängig davon ob ein weiterer Ring folgt. Zwar sind noch feinere Unterschiede zu beobachten, aber die Reichweite des Einflusses des Liganden nimmt mit zunehmendem Abstand vom Metallzentrum ab. Die Unterschiede zu den anderen Systemen zeigen deutlich, dass der chemische Charakter des Pyrazinrings eine entscheidende Rolle spielt. Seine Funktion, in abgeschwächter Form Elektronen 
zur Verfügung zu stellen, konnte eindeutig mittels der Bindungsanalyse belegt werden und zeigt sich auch anhand der Änderungen der Größen wie Bindungslängen, Ladungen oder Orbitalcharakter. Deutlich wird dies auch im Vergleich mit den anderen Systemen. Die aromatischen Verbindungen besitzen auch die Fähigkeit Elektronen weiterzuleiten, jedoch tun sie das in zu hohem Maße. Das Pyranylsystem, das über kein bzw. nur den aliphatischen Ring selbst als Rückgrat verfügt, ist bei einigen Eigenschaften den Pyrazin- und Molybdopterinverbindungen wiederum ähnlicher, da es, wenn auch in sehr geringem Ausmaß doch noch mittels des aliphatischen Ringes unterstützend wirken kann und scheinbar eine zu große Konjugation eher störend auf das System wirkt als eine zu geringe.

Bezugnehmend auf den Redoxvorgang ist damit ganz eindeutig die Rolle des Liganden sowie der non-innocence Charakter der Dithioleneinheit hervorzuheben. Die Auswirkungen der Veränderungen im Molekül bei Wechsel der Oxidationsstufe sind an Atomen die das Metall umgeben, deutlich erkennbar, selbst weiter entfernte Atome sind, wenn auch nur geringfügig, mit in den Vorgang involviert.

\subsection{Zusammenfassung und Ausblick}

Im Rahmen dieser Arbeit wurden Untersuchungen an verschiedenen Modellsystemen für das aktive Zentrum der Mo- und W-Kofaktoren durchgeführt. Dazu gehört die Synthese von Liganden, welche neben der Dithioleneinheit auch den Pyranring enthalten sowie theoretische Studien die Aufschluss über den Charakter des molybdopterinhaltigen Liganden geben.

Modellkomplexe für das Molybdopterin im allgemeinen beinhalten bis auf wenige Ausnahmen lediglich die Dithioleneinheit aber nicht den Pyranring, da vorwiegend katalytische Funktionen untersucht wurden. Die Arbeiten von Garner et al. die den 
Zugang zu einem molybdopterinhaltigen Liganden beinhalten, führten bisher nicht zum vollständigen Molybdän- bzw. Wolframkomplex.

In theoretischen Studien liegt das Hauptaugenmerk ebenfalls auf dem katalytischen Prozess und umfasst daher meist sehr kleine Modellkomplexe, die nur das Metallzentrum und die Dithioleneinheit beinhalten. Die genauere Funktion des Molybdpterinliganden bzw. seiner einzelnen Betandteile, ist bisher nicht untersucht worden. Diese Arbeit schließt diese Lücke.

Es konnte gezeigt werden, dass es möglich ist, Modellkomplexe, die neben der Dithioleneinheit auch über den Pyranring verfügen, zu synthetisieren. Dabei konnte der Zugang zu dem dafür benötigten Dithiocarbonat verbessert werden. Bezüglich der Untersuchung dieser Komplexe sind jedoch weitergehende Forschungsarbeiten nötig, vor allem in Bezug auf ihre strukturelle Charakterisierung sowie ihrer katalytischen Funktionen.

Die theoretische Behandlung von Modellsystemen, die den gesamten Molybdopterinliganden enthalten, konnten anhand ausgewählter Eigenschaften zur Aufklärung seiner Natur beitragen. Vergleiche der geometrischen Daten mit Kristallstrukturen zeigen eine gute Übereinstimmung mit den aus den DFT-Rechnungen erhaltenen Parametern.

Die vorliegenden Ergebnisse liefern klare Hinweise auf die Funktionen der verschiedenen Bestandteile des Molybdopterins. Deutlich sichtbar wird dies vor allem an den geometrischen Parametern, die im Zuge der Oxidation charakteristischen Trends unterliegen. So zeigen sich die stärksten Änderungen an den Metall-Schwefel- und Schwefel-Kohlenstoff-Bindungen, was auf den non-innocent-Charakter der Dithioleneinheit zurückzuführen ist. Die Veränderungen im Pyranring geben einen klaren Hinweis auf seine Funktion als Modulator der Konformation. Die in diesem Ring vorliegende C-C-Doppelbindung führt zu einer zusätzlichen Ringspannung, für die der 
Sauerstoff durch seine Position einen Ausgleich bietet. Die sich im Laufe der Oxidation verkürzende Bindung würde zu Abstoßungen führen, die sich aber durch die sich verstärkende endo-Position des Sauerstoffs verringern. Durch diesen Vorgang können sich die benachbarten C-Atome tetraedrisch und die C-C-Doppelbindung in den für sie günstigen Winkel von $120^{\circ}$ ausrichten und so die Ringspannung minimieren. Des weiteren bietet der Pyransauerstoff die Möglichkeit einer Ringöffnung, die eine enorme Flexibilität des gesamten Gerüsts zur Folge haben. Die durch elektronische Einflüsse auftretenden Geometrieänderungen werden somit durch den Pyranring abgepuffert. Ein elektronischer Einfluss des Pyransauerstoffs bzw. seiner freien Elektronenpaare wurde nicht beobachtet. Lediglich in den elektronenreichen aromatischen Systemen findet eine schwache Wechselwirkung des Aromaten mit einem freien Elektronenpaar des Sauerstoffs statt.

Bezüglich der von Inscore et al. aufgestellten oxo-gate-Hypothese finden sich Hinweise auf eine bindungsartige Wechselwirkung zwischen den zu einem Liganden gehörenden Schwefel-Atomen. Einerseits kommt es zu einer Veränderung in ihrer Bindungslänge, was auf eine zunehmende Wechselwirkung hindeutet, andererseits konnte anhand der NBO-Daten gezeigt werden, dass es definitiv Wechselwirkungen zwischen den Atomen gibt. Damit ergibt sich ein zusätzlicher Aspekt in den Eigenschaften des Dithiolens hinsichtlich zukünftiger Untersuchungen.

Neben elektronischen Eigenschaften wurden auch die Energiebarrieren des Oxidationsprozesses untersucht. Die beim Wechsel der Oxidationsstufen zu überwindende Energiebarriere gibt einen Einblick in das Verhalten beider Metalle und der unterschiedlichen Liganden im Vergleich. So ist bei allen Modellsystemen die OS VI die am schwersten erreichbare Stufe, wobei die Energiebarriere für Wolfram etwas tiefer liegt. Der Wechsel von OS IV zu OS V dagegen ist bei den Molybdänverbindungen einfacher und liegt energetisch insgesamt wesentlich niedriger. Die Oxoaffinitäten der 
Modellsysteme zeigen eine vielfach höhere Exergonität zugunsten des Wolframs. Diese Fakten spiegeln die Fähigkeit Wolframs, hohe Oxidationsstufen besser stabilisieren zu können, wider. In Bezug auf das natürliche Vorkommen der molybdän- und wolframhaltigen Enzyme stellt der gefundene Energieunterschied einen möglichen Grund dar.

Die unter hohen Temperaturen lebenden thermophilen Organismen, die über wolframhaltige Enzyme verfügen, können die höhere Aktivierungsenergie aufbringen. Während die mesophilen Organismen, die Molybdän in ihren aktiven Zentren eingebaut haben, bei hohen Temperaturen zu schnell reagieren würden bzw. nicht stabil wären, würden die Wolframzentren wiederum bei niedrigeren Temperaturen zu langsam bzw. überhaupt nicht reagieren.

Die erhaltenen Energieparameter der die Ringöffnung modellierenden Systeme geben einen ersten Einblick in den energetischen Ablauf dieses Prozesses. Die Größenordnung der Energieunterschiede zwischen offener und geschlossener Form bei den Modellsystemen des Pyrazins sowie der Übergangszustände zeigen, dass die Energiebarriere unter Berücksichtigung der im Enzym vorliegenden Umgebung durchaus überwindbar und damit eine Ringöffnung grundsätzlich möglich ist. Analog zu den Studien von Garner et al. in denen die verschiedenen Tautomere sowie die Protonierung des Pteridingerüsts mit einbezogen wurde, sind hier weitergehende Untersuchungen nötig. Vor allem die Berücksichtigung verschiedener zusätzlicher Faktoren wie Lösungsmitteleffekte, Protonierung des Systems sowie die Einflüsse der Seitenkette sollten dann eine Rolle spielen.

Das Pteridingerüst, das als stark desaktivierter Aromat mit schwachem $+\mathrm{M}$ und starkem -I-Effekt fungiert, ist in der Lage, dezidiert Elektronendichte an das Zentrum abzugeben bzw. aufzunehmen. Die Stellung der Stickstoffatome ermöglicht eine weitreichende Konjugation der $\pi$-Elektronen, die bis in das Metallzentrum hinein führt. 
Die als Modelle dienenden Chromanyl- und Flavanylverbindungen sind mit ihrem aromatischen Gerüst ebenfalls zu Konjugation befähigt, geben aber zuviel Elektronendichte weiter, so dass wie an den Fällen der Molybdänverbindungen in OS IV beobachtet, eine destabilisierende Wirkung auf das Metallzentrum zur Folge haben. Hier verdeutlicht sich die vom Pteridin ausgehende Feinabstimmung der elektronischen Interaktion.

Auch bei der Betrachtung weiterer Eigenschaften zeigt sich deutlich, dass der Molybdopterinligand im Vergleich zu den übrigen Modellverbindungen ein anderes Verhalten aufweist. Die Änderungen wichtiger Geometrieparameter während der Oxidation, wie z.B. der M-S-, C-C-Doppelbindungs-, S-C-Abstände sowie des Faltungswinkels sind beim Molybdopterinkomplex am geringsten.

Die Energieunterschiede bei dem Übergang von OS IV zu V liegen hier zwischen denen der Pyranylverbindungen, welche wesentlich höher sind und denen der aromatischen Systeme, welche dagegen niedriger sind. Die Orbitalkoeffizienten der Metallatome weisen bei den Molybdopterinverbindungen nahezu gleiche Werte für Molybdän und Wolfram auf, wobei bei allen anderen Systemen ein Unterschied in den Werten der Orbitalkoeffizienten zugunsten Wolframs vorliegt.

Die Synthese von Modellverbindungen mit dem vollständigen Molybdopterin-Liganden muss also einer der vordringlichen Ziele auf diesem Gebiet sein, auch wenn es eine enorme Herausforderung darstellt.

\subsection{Summary}

This work is dedicated to the investigation of model systems mimiking the active site of Mo and W cofactors. This study addresses two aspects of this topic which are the synthesis of ligands containing the dithiolene unit as well as the pyrane ring and 
theoretical investigations aimed towards the understandings of them.

Most model complexes known to date were prepared with the catalytic activity in mind and therefore nearly all of them contain the dithiolene unit but are lacking the pyrane ring. The first synthetic approach of molybdopterine containing ligands made by Garner did not lead to complete molybdenum and tungsten compounds.

Earlier theoretical studies due to their focus on catalysis were also mainly about small complexes composed of a metal centre and the dithiolene unit. The function of the molypdopterine ligand and its functional groups was neglected. The results of this work are a step towards closing this gap.

It was shown that the preparation of model substances containing the dithiolenene unit and the pyrane ring is possible. In the course of this work the route to the crucial dithio carbonates was optimized. The complete experimental examination of this complexes especially their structural characterisation and catalytic activity was beyond the scope of this work and leaves a field wide open to further research.

The ab initio treatment of model systems containing the whole molybdopterine ligand made his nature visible. The quality of this calculations was shown by direct comparison of the data obtained via X-ray cristallography and calculated values.

The following results clearly demonstrate the individual functions of the different moieties within the molybtopterine. The most pronounced effect is visible looking at the geometrical parameters which follow characteristic trends during the oxidative circle. The metal-sulfur and carbon-sulfur bons undergo the most pronounced changes. This behaviour can be linked to the non-innocent character of the dithiolene unit. The change within the pyrane ring point at its role as a conformational modulator. The additional strain, caused by its $\mathrm{C}-\mathrm{C}$ double bond, can be compensated by the oxygen changing its position. This becomes obvious when one considers the oxidation process leading to a bond shortening which should cause an increased repulsion. This 
repulsive effect is moderated due to the fact that the oxygen's endo position becomes more pronounced too. The ring strain is thus minimized because now it is possible for the neighbouring carbon atoms to obtain a tetraedric geometry and for the C-C double bonds to reach the favoured $120^{\circ}$ angle. Another probable advantage provided by the oxygen within the pyrane is the possible ring opening causing an enormous flexibility of the backbone. The influence of electronic effects on the overall geometry is thus counterbalanced through the pyrane ring. With the exception of the electron rich aromatic systems where the free electron pair of the oxygen shows slight interactions neither the oxygen nor its free electron pair causes any electronic effects.

Inscore et al. proposed the so called oxo-gate-hypothesis for which we found some reasoning based on a bonding like interaction between the sulfur atoms belonging to one dithiolene ligand. There is a change in the bond length pointing towards an increase in interaction. This was proven by the NBO analysis which definetely showed that there are interactions between the atoms. This additional feature of the dithiolene should be researched in more detail in following investigations.

In addition to the studies on the electronic properties the energy barriers of the oxidation process were also examined. By looking at the barrier height between the different oxidation states it is possible to compare both metals and the ligands directly. It can be stated that for all model-systems it is most difficult to reach OS VI and that this is easier for tungsten. The change from OS IV to OS V is easier in the case of the molybdenum compounds and the barrier height is here significantly lower. The oxoaffinities of all model systems show a much higher exergony in favour of tungsten.

All those facts demonstrate the better ability of tungsten to stabilize higher oxidation states. This energetic differences may be the reason for the observed distribution of molybdenum and tungsten containing enzymes in nature. The thermophilic organism are able to employ tungsten containing enzymes because the high temperature 
provides enough energy for the higher amount of activation energy needed. The mesophilic organisms utilize molybdenum in their active centres which would be to reactive or even become instable at higher temperature compared to tungsten centres which loose their reactivity at lower temperatures.

The parameters obtained for the energetics of those systems which model the ring opening make it possible to obtain a first picture of the whole process. The differences in energy between the closed and the open form of the pyrazine model system and the transition states indicate that within the environment of the enzyme the barrier height does not prevent ring opening. In accordance with the studies performed by Garner et al. which investigated different tautomers and the protonation of the pteridine-backbone additional studies should be conducted. For are real extension of the results already presented here factors like solvent, protonation and the influence of the side chain must be considered.

The pteridine backbone acting as a strongly deactivated aromatic system showing a weak $+\mathrm{M}$ und a strong -I effect is able to transfer electronic density to and from the centre. The position of the nitrogen atoms makes a conjugation of the $\pi$-electrons possible which reaches deep into the metal centre. This effect is also possible in the case of the chromanyl and flavanyl compounds but their aromatic system, as can be seen for the molybdenum compounds in oxidation state IV, transfers so much electronic density that a destabilisation of the metal centre occurs. This clearly shows how balanced and delicate the electronic interaction achieved by the pteridine actually is.

The consideration of further characteristics of the molybdopterine ligand in comparison to the other model systems clearly shows its different behaviour. The changes of the most important geometrical parameters as the M-S, the C-C, the S-C distances and the folding angle during the oxidation process are smallest for the molybdopterine 
complex.

Going from OS IV to V its energetical differences are between those of the pyranyl compounds and the aromatic systems. The orbital coefficients of the metal atoms show nearly no difference between molybdenum and tungsten in the case of the molybdopterine compound while in all other systems those values show a difference favouring tungsten.

Despite being highly ambitious the most crucial objective in this research field is therefore the synthesis of the complete molybdopterine model system. 


\section{Kapitel 3}

\section{Experimenteller Teil}

\subsection{DFT-Rechnungen}

Für die behandelten Modellverbindungen wurden DFT-Rechnungen ausgeführt. Für alle Rechnungen wurde das Drei-Parameter-Hybridfunktional von Becke (B3) [136] und das Korrelationsfunktional von Lee, Yang und Parr (LYP) [137] angwendet. Für die Metallatome Molybdän und Wolfram wurde der Basissatz LANL2DZ [138] und für die übrigen Atome 6-311G(d,p) [138] verwendet. Die Basis LANL2DZ beinhaltet die effektiven Kernpotentiale (Los Alamos) mit DZ-Funktionen für Metalle ab der zweiten Übergangsmetallreihe. Die Basis 6-311G(d,p) besteht aus der 6-311G-Basis erweitert um zusätzliche Polarisationsfunktionen.

Für die natürliche Populationsanalyse wurden die Ladungen nach dem NBOFormalismus 1 analysiert. Die Strukturen wurden ohne Symmetrieeinschränkungen volloptimiert, als Konvergenzkriterium gilt dabei eine Änderung von unter $1 \cdot 10^{-8}$.

Eine allein auf den ab inito Wellenfunktionen basierende Beschreibung der Orbitale gibt die Bindungsverhältnisse im Rahmen des gewählten Ansatzes zwar quantitativ korrekt wieder, entspricht jedoch nicht dem in der Chemie üblichen qualitativen Bild

\footnotetext{
${ }^{1}$ natural bond orbital formalism
} 
einer chemischen Bindung. Für die Beschreibung der Bindung wurde daher eine weiterführende Analyse durchgeführt. Die NBO-Analyse [139, 140, 141, 142] ermöglicht es, die aus der ab initio Rechnung erhaltenen delokalisierten Molekülorbitale in die zugrundeliegenden Lewis-Strukturen zu zerlegen und somit auch die Beschreibung der Bindungsverhältnisse.

Die Zerlegung der Gesamtwellenfunktion in die optimalen atomaren Beiträge (natural atomic orbitals NAO) [143, 144] 2] ermöglicht es, jedem Atom seinen Anteil an der Gesamtelektronenzahl zuzuweisen (Natural Population Analysis, NPA) und somit unter Berücksichtigung der Kernladung (KLZ) eine direkte Angabe der Partialladung. Die Summe aller Partialladungen muss der Gesamtladung entsprechen.

Der von K. B. Wiberg eingeführte Bond-Index (WBI) [145, 146] 3 weist den großen Vorteil auf, dass er weitgehend unabhängig von dem verwandten Basissatz ist und sein aus der Populationsanalyse erhaltener Wert ungefähr der dem Chemiker geläufigen Bindungsordnung entspricht.

Der Betrag des Orbitalkoeffizienten $c_{i j}$ stellt ein Maß für die Beteiligung eines atomaren Orbitals an der Gesamtwellenfunktion und sein Vorzeichen die Art der Wechselwirkung (bindend oder antibindend) dar [147]. Für die Darstellung eines Molekülorbitals als Linearkombination von $\mathrm{n}$ Atomorbitalen gilt:

$$
\Psi_{i}=\sum_{j=1}^{n} c_{i j} \cdot \psi_{j} \text { mit } \sum_{j=1}^{n} c_{i j}^{2}=1
$$

Für alle Rechnungen wurde das Programmpaket Gaussian03 [148] sowie die darin enthaltenen Prozeduren verwendet. Die Übergangszustände wurden mit dem Programmpaket Turbomole V 5.9 [149] berechnet. Die Visualisierung der Molekülstrukturen sowie der Orbitale erfolgte mit den Programmen Molden [150] und Molekel [151].

\footnotetext{
${ }^{2}$ Genau gesagt ergeben sich die NAOs als lokale Eigenvektoren der reduzierten EinteilchenDichtefunktion und sind somit eine intrinsische Eigenschaft der Wellenfunktion selbst

${ }^{3}$ Der WBI wird als Summe der Quadrate der Dichtematrixelemente der an der Bindung beteiligten Atome berechnet und ist grundstzlich positiv definiert.
} 


\section{$3.2 \quad$ Experimentelles}

Alle Reaktionen wurden in ausgeheizten und im Vakuum abgekühlten Glasgeräten unter einer sauerstofffreien Stickstoffatmosphäre durchgeführt. Die verwendeten Lösungsmittel wurden nach Literaturvorschriften getrocknet und mit Stickstoff gesättigt[organikum].

Schmelzpunkte wurden in abgeschlossenen Glaskapillaren mit dem Gerät Büchi Melting Point B-540 bestimmt.

NMR-Spektren wurden auf den Geräten Bruker und Avance 200 und 500, Bruker AM 250 und Bruker AS 400 bestimmt. Als externer Standard wurde TMS $(H)$ verwendet. Heterokern-NMR-Spektren wurden ${ }^{1} \mathrm{H}$ - breitbandentkoppelt aufgenommen. Verwendete Lösungsmittel und Betriebsfrequenzen sind bei den Versuchsbeschreibungen aufgeführt. Die Multiplizitäten sind wie folgt abgekürzt: $s=$ Singulett, $d=D u-$ blett, $\mathrm{t}=$ Triplett, $\mathrm{m}=$ Multiplett. Die chemischen Verschiebungen $\delta$ sind in ppm angegeben, wobei negative Vorzeichen eine Hochfeldverschiebung und positive Vorzeichen eine Tieffeldverschiebung bezogen auf den jeweiligen Standard bedeuten. Die Messungen erfolgten bei Raumtemperatur.

Massenspektren wurden mit den Geräten Varian MAT CH5, Finnigan MAT 95 und Finnigan MAT System 8230 aufgenommen. In allen Fällen wurde ElektronenstoßIonisation (EI) bei $70 \mathrm{eV}$ als Ionisationsmethode verwendet. Es wird der stärkste zu einer Isotopenverteilung gehörende Peak mit seiner relativen prozentualen Intensität angegeben.

IR-Spektren wurden auf einem BIO-RAD Digilab FTS 7 Spektrometer aufgenommen. Alle Substanzen wurden als KBr-Pressling gemessen. Die Bandenintensitäten sind wie folgt abgekürzt: $\mathrm{st}=$ stark, $\mathrm{m}=$ mittel, $\mathrm{s}=$ schwach.

Elementaranalysen wurden vom Analytischen Labor des Instituts für Anorganische Chemie der Universität Göttingen auf dem Gerät C,H,N-Rapid der Firma 
Heraeus durchgeführt.

\subsection{Darstellung der Ausgansverbindungen}

Die Ausgangsverbindungen waren im Handel erhältlich oder wurden nach Literaturvorschriften hergestellt:

Tetrahydro-4H-pyran-4-on[[152]], $\mathrm{K}_{3} \mathrm{Na}\left[\mathrm{MoO}_{2}(\mathrm{CN})_{4}\right]^{*} 6 \mathrm{H}_{2} \mathrm{O}[[153]], \mathrm{K}_{3} \mathrm{Na}\left[\mathrm{WO}_{2}(\mathrm{CN})_{4}\right]-$ $* 6 \mathrm{H}_{2} \mathrm{O}[[154]]$.

\subsubsection{Darstellung von 3-Bromo-2,3-dihydro-4 $\boldsymbol{H}$-chroman-4-on}

$\mathrm{Zu}$ einer Lösung von Chromanon (5.35 g, $36.11 \mathrm{mmol}, 1.00$ Äq.) und $\mathrm{K}_{2} \mathrm{CO}_{3}$ (5.14 g, 37.19 mmol, 1.03 Äq.) in $500 \mathrm{~mL} \mathrm{CCl}$ gab man $1.66 \mathrm{~mL} \mathrm{Br}_{2}(5.19 \mathrm{~g}, 32.50 \mathrm{mmol}$, 0.90 Äq.) und rührte bis zur vollständigen Entfärbung. Das Gemisch wurde filtriert und das Filtrat i.V. (im Vakuum) eingeengt. Der verbleibende Rückstand wurde mit $\mathrm{CH}_{2} \mathrm{Cl}_{2}$ ( 3 x $50 \mathrm{~mL}$ ) extrahiert und mit je $3 \times 20 \mathrm{~mL}$ Wasser, ges. $\mathrm{NaHCO}_{3}$ und NaCl-Lösung gewaschen. Die Phasen wurden getrennt und die organische Phase über $\mathrm{MgSO}_{4}$ getrocknet. Das Lösungsmittel wurde i.V. entfernt und das Rohprodukt durch Umkristallisation in Ethanol gereinigt. Die erhaltene Zielverbindung kristallisiert bei Raumtemperatur mit einer Ausbeute von $72 \%$ (5.88 g, $25.90 \mathrm{mmol}$ ).

Schmelzpunkt: $88^{\circ} \mathrm{C}$.

${ }^{1} \mathrm{H}-\mathrm{NMR}\left(300.13 \mathrm{MHz}, \mathrm{CDCl}_{3}\right): \delta=4.55-4.72(\mathrm{~m}, 3 \mathrm{H}, 1-\mathrm{H}, 2-\mathrm{H}), 7.00-7.10(\mathrm{dt}$, $2 \mathrm{H}$, arom. 4-H, 5-H, J = 8.4, $14.2 \mathrm{~Hz}), 7.50-7.55(\mathrm{dt}, 1 \mathrm{H}$, arom 6-H, 7.25, $1.8 \mathrm{~Hz})$, 7.91 - $7.94(\mathrm{dd}, 1 \mathrm{H}$, arom 3-H, 0.4, 7.9 Hz).

${ }^{13} \mathrm{C}-\mathrm{NMR}\left(75.5 \mathrm{MHz}, \mathrm{CDCl}_{3}\right): \delta=45.35\left(1 \mathrm{C}, C \mathrm{H}_{2}\right), 71.26(1 \mathrm{C}, C \mathrm{HBr}), 117.92$, 122.32, 128.25, 136.71 (4 C, arom. C), 160.64 (1 C, arom. C-O), 185.19 (1 C, CO). 
MS: m/e $(\%)=226\left[\mathrm{M}^{+}, 20\right], 120[\mathrm{M}-\mathrm{Br}, \mathrm{CH} 2, \mathrm{O}, 100]$.

Elementaranalyse für $\mathrm{C}_{9} \mathrm{H}_{7} \mathrm{BrO}_{2}(227.05 \mathrm{~g} / \mathrm{mol})$

ber. C 47.61, H 3.11

gef. C 47.60, H 3.14

\subsubsection{O-Ethyl-S-(4-oxo-3,4-dihydro-2 H-chromanon-3-yl)dithio- carbonat}

Zu einer Lösung von 3-Bromo-2,3-dihydro-4 $H$-chroman-4-on (3.00 g, 13.21 mmol, 1.00 Äq.) in $25 \mathrm{~mL}$ Methanol wurde Kalium-O-ethylxanthogenat (1.96 g, $13.21 \mathrm{mmol}, 1.00$ Äq.) in $60 \mathrm{ml}$ Methanol zugegeben. Das Gemisch wurde $30 \mathrm{~min}$ bei $50{ }^{\circ} \mathrm{C}$ gerührt und das Lösungsmittel nach Abkühlen auf RT i.V. entfernt. Nach Versetzen des gelben Rückstands mit $10 \mathrm{~mL} \mathrm{H}_{2} \mathrm{O}$ wurden $10 \mathrm{~mL} 10 \% \mathrm{HCl}$ zugegeben und die Lösung 10 min gerührt. Die Suspension wurde mit 3 x $30 \mathrm{~mL}$ Diethylether extrahiert. Die organische Phase wurde mit Wasser $(2 \times 50 \mathrm{~mL})$ und gesättigter NaCl-Lösung $(2 \times 50$ $\mathrm{mL}$ ) gewaschen und über $\mathrm{MgSO}_{4}$ getrocknet. Das Lösungsmittel wurde i.V. entfernt und das Rohprodukt aus Ethanol umkristallisiert. Die Verbindung wurde als gelber kristalliner Feststoff in 59\% Ausbeute erhalten (2.09 g, $7.79 \mathrm{mmol})$.

Schmelzpunkt: $78{ }^{\circ} \mathrm{C}$

${ }^{1} \mathrm{H}-\mathrm{NMR}\left(300.13 \mathrm{MHz}, \mathrm{CDCl}_{3}\right): \delta=1.19\left(\mathrm{t}, 3 \mathrm{H}, \mathrm{CH}_{3}\right), 2.80\left(\mathrm{q}, 2 \mathrm{H}, \mathrm{CH}_{2}\right), 4.48$ $(\mathrm{m}, 1 \mathrm{H},-\mathrm{SCH}), 4.58-4.75\left(\mathrm{~m}, 1 \mathrm{H}, \mathrm{OCH}_{2}\right), 4.90-5.08\left(\mathrm{~m}, 1 \mathrm{H}, \mathrm{OCH}_{2}\right), 6.90-7.04(\mathrm{~m}$, $2 \mathrm{H}$, arom. H), 7.38-7.57 (m, $1 \mathrm{H}$, arom. H), 7.90-8.07 (m, $1 \mathrm{H}$, arom. H).

Elementaranalyse für $\mathrm{C}_{12} \mathrm{H}_{12} \mathrm{O}_{3} \mathrm{~S}_{2}(268.35 \mathrm{~g} / \mathrm{mol})$

ber. C 53.71, H 4.51

gef. C 53.55, H 4.71

MS: m/e (\%) $=268$ [M, 36], 239 [M -Ethyl, 52], 208 [M -Ethyl, -S, 100]. 


\subsection{3 $4 H$-[1,3]Dithiolo[4,5-c]chroman-2-on}

Innerhalb von $10 \mathrm{~min}$ wurden unter Rühren $30 \mathrm{~mL}$ trockene $45 \%$ ige $\mathrm{HBr} / \mathrm{AcOH}$ zu 2.00 g Chromanonxanthogenat $(7.45 \mathrm{mmol})$ gegeben. Nach $12 \mathrm{~h}$ weiterem Rühren wurde die Lösung mit Eiswasser versetzt. Nach Erwärmen auf RT wurde der ausgefallenen Feststoff abfiltriert, mit Wasser gewaschen und i.V. getrocknet. Umkristallisation in Ethanol ergab $0.53 \mathrm{~g}(2.38 \mathrm{mmol}, 32 \%)$ der Verbindung in Form eines weißen bei RT kristallisierenden Feststoffes.

Schmelzpunkt: $82^{\circ} \mathrm{C}$

${ }^{1} \mathrm{H}-\mathrm{NMR}\left(300.13 \mathrm{MHz}, \mathrm{CDCl}_{3}\right): \delta=5.02\left(\mathrm{~s}, 2 \mathrm{H}, \mathrm{CH}_{2}\right), 6.88-6.98(\mathrm{~m}, 2 \mathrm{H}$, arom. H), 7.15- 7.22 (m, $2 \mathrm{H}$, arom. H).

${ }^{13} \mathrm{C}-\mathrm{NMR}\left(75.5 \mathrm{MHz}, \mathrm{CDCl}_{3}\right): \delta=64.01(1 \mathrm{C}, \mathrm{C}=C), 64.03(1 \mathrm{C}, C=\mathrm{C}), 116.79$, 122.55, 124.51, 129.94 (4 C, arom. C), $152.33\left(1 \mathrm{C}, C \mathrm{H}_{2}\right), 190.01(1 \mathrm{C}, C=\mathrm{O})$.

Elementaranalyse für $\mathrm{C}_{10} \mathrm{H}_{6} \mathrm{O}_{2} \mathrm{~S}_{2}(222.28 \mathrm{~g} / \mathrm{mol})$

ber. C 54.03, H 2.72, S 28.85

gef. C 53.78, H 2.29, S 28.44

MS: $\mathrm{m} / \mathrm{e}(\%)=222[\mathrm{M}, 100]$.

IR (KBr, $\left.\mathrm{cm}^{-1}\right) \nu=3070(\mathrm{~s}), 3023(\mathrm{~s}), 2963(\mathrm{~s}), 2926(\mathrm{~s}), 2872(\mathrm{~s}), 1718(\mathrm{~m}), 1667(\mathrm{st})$, 1655(st), 1619(st), 1560(s), 1488(m), 1454(m), 1261(m), 1232(m), 1154(s), 1101(m), 1041(m), 1010(m), 928(s), 899(s), 855(s), 818(m), 791(m), 746(st), 651(m), 622(s), 543(s), 509(s), 441(s), 433(s), 375(s), 327(s).

\subsection{4 $\left[\mathrm{PPh}_{4}\right]_{2}[\mathrm{MoO}(\mathrm{cdt})]_{2}$}

0.20 g $4 H$-[1,3]Dithiolo[4,5-c]chroman-2-on (0.90 mmol, 2.00 Äq.) wurden in $15 \mathrm{~mL}$ Ethanol mit $0.05 \mathrm{~g} \mathrm{KOH} \mathrm{(0.90} \mathrm{mmol,} 2.00$ Äq.) versetzt und $30 \mathrm{~min}$ bei $30{ }^{\circ} \mathrm{C}$ gerührt. Das Gemisch wurde zu einer Lösung von $\mathrm{K}_{3} \mathrm{Na}\left[\mathrm{MoO}_{2}(\mathrm{CN})_{4}\right]^{*} 6 \mathrm{H}_{2} \mathrm{O}(0.15 \mathrm{~g}, 0.45$ mmol, 1.00 Äq.) und $\mathrm{KOH}\left(0.10 \mathrm{~g}, 1.80 \mathrm{mmol}, 4.00\right.$ Äq.) in 15 mL $\mathrm{H}_{2} \mathrm{O}$ gegeben und $3 \mathrm{~h}$ 
auf $70{ }^{\circ} \mathrm{C}$ erhitzt. Nach Abkühlen der Reaktionslösung auf RT wurde eine Lösung aus $\mathrm{PPh}_{4} \mathrm{Cl}$ (0.35 g, $0.90 \mathrm{mmol}, 2.00$ Äq.) in $15 \mathrm{~mL}$ Ethanol zugegeben. Das ausgefallene Produkt $(0.15 \mathrm{~g}, 28 \%)$ wurde abfiltriert, mit Wasser $(3 \mathrm{x} 10 \mathrm{~mL})$ gewaschen und i.V. getrocknet.

Schmelzpunkt: $175^{\circ} \mathrm{C}$ Zersetzung

Elementaranalyse für $\mathrm{C}_{66} \mathrm{H}_{52} \mathrm{MoO}_{3} \mathrm{P}_{2} \mathrm{~S}_{4}(1179.26 \mathrm{~g} / \mathrm{mol})$

ber. C $67.22, \mathrm{H} 4.44, \mathrm{~S} 10.88$

gef. C $63.34, \mathrm{H} 4.19, \mathrm{~S} 10.60$

$\operatorname{IR}\left(\mathrm{KBr}, \mathrm{cm}^{-1}\right) \nu=3055(\mathrm{~s}), 2964(\mathrm{~s}), 2851$ (s), 2816 (s), 2053 (s), 1827 (s), 1685 (s), $1648(\mathrm{~s}), 1585(\mathrm{~m}), 1550(\mathrm{~m}), 1483(\mathrm{~m}), 1437(\mathrm{~m}), 1361$ (s), $1314(\mathrm{~m}), 1299(\mathrm{~m})$, 1187 (s), 1160 (s), 1108 (st), 1022 (m), 996 (m), 918 (s), 852 (s), 802 (s), $756(\mathrm{~m}), 722$ (st), $690(\mathrm{~m}), 607(\mathrm{~s}), 527$ (st).

\subsection{5 $\left[\mathrm{Ph}_{6} \mathrm{NP}_{2}\right]_{2}[\mathrm{WO}(\mathrm{cdt})]_{2}$}

0.30 g $4 H$-[1,3]Dithiolo[4,5-c]chroman-2-on (1.35 mmol, 2.00 Äq.) wurden in $15 \mathrm{~mL}$ Ethanol mit $0.08 \mathrm{~g} \mathrm{KOH} \mathrm{(mmol,} 2.00$ Äq.) versetzt und $30 \mathrm{~min}$ bei $30{ }^{\circ} \mathrm{C}$ gerührt. Das Gemisch wurde zu einer Lösung von $\mathrm{K}_{3} \mathrm{Na}\left[\mathrm{WO}_{2}(\mathrm{CN})_{4}\right]^{*} 6 \mathrm{H}_{2} \mathrm{O}(0.29 \mathrm{~g}, 0.68 \mathrm{mmol}$, 1.00 Äq.) und $\mathrm{KOH}\left(0.15 \mathrm{~g}, 2.70 \mathrm{mmol}, 4.00\right.$ Äq.) in $15 \mathrm{~mL} \mathrm{H}_{2} \mathrm{O}$ gegeben und $3 \mathrm{~h}$ auf $70{ }^{\circ} \mathrm{C}$ erhitzt. Nach Abkühlen der Reaktionslösung auf RT wurde eine Lösung aus $\mathrm{Ph}_{6} \mathrm{NP}_{2} \mathrm{Cl}$ (0.77 g, $1.35 \mathrm{mmol}, 2.00$ Äq.) in $15 \mathrm{~mL}$ Ethanol zugegeben. Das ausgefallene Produkt $(0.26 \mathrm{~g}, 23 \%)$ wurde abfiltriert, mit Wasser $(3 \times 10 \mathrm{~mL})$ gewaschen und i.V. getrocknet.

Schmelzpunkt: $188^{\circ} \mathrm{C}$ Zersetzung

Elementaranalyse für $\mathrm{C}_{90} \mathrm{H}_{72} \mathrm{~N}_{2} \mathrm{WO}_{3} \mathrm{P}_{4} \mathrm{~S}_{4}(1665.54 \mathrm{~g} / \mathrm{mol})$

ber. C $64.90, \mathrm{H} 4.36,7.70$

gef. C $66.92, \mathrm{H} 5.78, \mathrm{~S} 6.52$ 
$\operatorname{IR}\left(\mathrm{KBr}, \mathrm{cm}^{-1}\right) \nu=3413(\mathrm{~s}), 3364$ (s), 3056 (s), 2963 (s), 2204 (s), 1918 (s), 1831 (s), $1685(\mathrm{~s}), 1604(\mathrm{~s}), 1585(\mathrm{~m}), 1540(\mathrm{~s}), 1483(\mathrm{~m}), 1437(\mathrm{~m}), 1314(\mathrm{~s}), 1298(\mathrm{~s})$, $1238(\mathrm{~m}), 1187$ (s), 1161 (s), 1107 (st), 1022 (m), 996 (m), 948 (s), 904 (s), 853 (s), $815(\mathrm{~s}), 754(\mathrm{~m}), 722(\mathrm{st}), 689$ (st), $615(\mathrm{~s}), 605(\mathrm{~s}), 526(\mathrm{st}), 447(\mathrm{~s})$.

\subsubsection{8-Oxa-1,4-dithiaspiro[4.5]dekan}

Zu 2.00 g Tetrahydropyranon (2.2 mL, $20 \mathrm{mmol}, 1.00$ Äq.) wurden 1,2-Ethanthiol (3.36 mL, $40 \mathrm{mmol}, 2.00$ Äq.), $\mathrm{BF}_{3}{ }^{*} \mathrm{OEt}_{2}$ (2.45 mL, $20 \mathrm{mmol}, 1.00$ Äq.) sowie Essigsäure (1.14 mL, $20 \mathrm{mmol}, 1,00$ Äq.) gegeben. Die hellrote Lösung wurde $1 \mathrm{~h}$ bei Raumtemperatur gerührt. Das Gemisch wurde mit $30 \mathrm{~mL}$ Hexan überschichtet und mit je 3 x $20 \mathrm{~mL}$ gesättigter $\mathrm{NaHCO}_{3}$-Lösung, $10 \% \mathrm{NaOH}$-Lösung und gesättigter NaCl-Lösung gewaschen. Die organische Phase wurde i.V. getrocknet. Das erhaltene farblose Öl (3.17 g, 18 mmol, 89\%) wurde aufgrund seiner Empfindlichkeit ohne weitere Reinigung als Rohprodukt weiterverwendet.

${ }^{1} \mathrm{H}-\mathrm{NMR}\left(300.13 \mathrm{MHz}, \mathrm{CDCl}_{3}\right): \delta=2.03\left(\mathrm{t}, 4 \mathrm{H}, \mathrm{CH}_{2}\right), 3.23\left(\mathrm{~s}, 4 \mathrm{H}, \mathrm{S}-\mathrm{CH}_{2}\right), 3.68$ $\left(\mathrm{t}, 4 \mathrm{H}, \mathrm{O}-\mathrm{CH}_{2}\right)$.

${ }^{13} \mathrm{C}-\mathrm{NMR}\left(75.5 \mathrm{MHz}, \mathrm{CDCl}_{3}\right): \delta=38.32\left(2 \mathrm{C}, C \mathrm{H}_{2}\right), 42.69\left(2 \mathrm{C}, \mathrm{S}-C \mathrm{H}_{2}\right), 65.20$

$\left(1 \mathrm{C}, \mathrm{C}_{\text {quart }}\right), 67.27\left(2 \mathrm{C}, \mathrm{O}-\mathrm{CH}_{2}\right)$.

MS: $\mathrm{m} / \mathrm{e}(\%)=176[\mathrm{M}, 100], 118$ [M -Ethyl, -S, 53].

\subsubsection{2,3,7,8-Tetrahydo-5H-[1,4]dithiino[2,3-c]pyran}

$\mathrm{Zu}$ 8-Oxa-1,4-dithiaspiro[4.5]dekan (3.17 g, $18 \mathrm{mmol}, 1.00$ Äq.) in $25 \mathrm{~mL} \mathrm{CH}_{2} \mathrm{Cl}_{2}$ wurde unter Eiskühlung innerhalb von 5 min NBS (3.20 g, $18 \mathrm{mmol}, 1.00$ Äq.) in $25 \mathrm{~mL} \mathrm{CH}_{2} \mathrm{Cl}_{2}$ gegeben. Nach 30 min Rühren bei RT wurde die Lösung mit $50 \mathrm{~mL}$ Hexan verdünnt und mit 3 x 30 mL ges. $\mathrm{NaHCO}_{3}$-Lösung, $\mathrm{H}_{2} \mathrm{O}$ und gesättigter $\mathrm{NaCl}$ Lösung gewaschen. Die organische Phase wurde abgetrennt und das Lösungsmittel 
i.V. entfernt. Das Rohprodukt (1.94 g, 62\%) wurde als rotes Öl erhalten und aufgrund seiner Empfindlichkeit ohne Reinigung weiter umgesetzt.

${ }^{1} \mathrm{H}-\mathrm{NMR}\left(300.13 \mathrm{MHz}, \mathrm{CDCl}_{3}\right): \delta=2.08\left(\mathrm{t}, 2 \mathrm{H}, \mathrm{CH}_{2}\right), 3.29\left(\mathrm{~s}, 4 \mathrm{H}, \mathrm{S}-\mathrm{CH}{ }_{2}\right), 3.73$ $\left(\mathrm{t}, 2 \mathrm{H}, \mathrm{O}-\mathrm{CH}_{2}\right), 3.95\left(\mathrm{~s}, 2 \mathrm{H}, \mathrm{O}-\mathrm{CH}_{2}\right)$.

\subsection{8 $\left[\mathrm{PPh}_{4}\right]_{2}[\mathrm{MoO}(\mathrm{pdt})]_{2}$}

Zu Dithiin (1.94 g, 11 mmol, 2.00 Äq.) in $20 \mathrm{~mL}$ Ethanol wurde $0.88 \mathrm{~g} \mathrm{NaOH}(22$ mmol, 2.00 Äq.) gegeben und 30 min bei $40{ }^{\circ} \mathrm{C}$ gerührt, wobei sich die Lösung braun färbte. Das Reaktionsgemisch wurde zu einer Lösung aus 1.87 g, (6 mmol 1.00 Äq.) $\mathrm{K}_{3} \mathrm{Na}\left[\mathrm{MoO}_{2}(\mathrm{CN})_{4}\right]^{*} 6 \mathrm{H}_{2} \mathrm{O}$ und $1.76 \mathrm{~g} \mathrm{NaOH}$ (44 mmol, 4.00 Äq.) in $20 \mathrm{~mL} \mathrm{H} \mathrm{H}_{2} \mathrm{O}$ gegeben und $2 \mathrm{~h}$ auf $65{ }^{\circ} \mathrm{C}$ erhitzt. Nach Abkühlen auf RT wurden $8.25 \mathrm{~g} \mathrm{PPh}_{4} \mathrm{Cl}(22$ mmol, 2.00 Äq.) in 20 mL Ethanol zugegeben. Das ausfallende Produkt (3.87 g, 65\%) wurde abfiltriert, mit $3 \times 10 \mathrm{~mL} \mathrm{H}_{2} \mathrm{O}$ gewaschen und i.V. getrocknet.

Schmelzpunkt: $188-190{ }^{\circ} \mathrm{C}$ Zersetzung

Elementaranalyse für $\mathrm{C}_{58} \mathrm{H}_{52} \mathrm{MoO}_{3} \mathrm{P}_{2} \mathrm{~S}_{4}(1083.18 \mathrm{~g} / \mathrm{mol})$

ber. C $64.31, \mathrm{H} 4.84$, S 11.84

gef. C $65.52, \mathrm{H} 5.11, \mathrm{~S} 9.49$

IR $\left(\mathrm{KBr}, \mathrm{cm}^{-1}\right) \nu=3055(\mathrm{~m}), 2951(\mathrm{~m}), 2926(\mathrm{~m}), 2847(\mathrm{~m}), 2707(\mathrm{~s}), 2612(\mathrm{~s})$, $2214(\mathrm{~s}), 2116$ (s), 2055 (s), 1974 (s), 1904 (s), 1836 (s), 1814 (s), 1776 (s), 1680 (m), 1590 (m), 1484 (m), 1463 (m), 1438 (st), 1382 (m), 1332 (m), 1311 (m), 1277 (m), 1235 (m), 1193 (st), 1120 (st), 1099 (st), 1071 (st), 1027 (m), 996 (m), 924 (m), 858 (m), $836(\mathrm{~m}), 751(\mathrm{~m}), 721$ (st), 695 (st), 617 (s), 543 (m), 504 (st), 457 (m), 443 (m), $396(\mathrm{~s}), 300(\mathrm{~m}), 290(\mathrm{~m})$. 


\subsection{Behandlung und Entsorgung der Abfälle}

Die eingesetzten Lösungsmittel wurden abdestilliert oder im Vakkum einkondensiert und in die dafür vorgesehenen Behälter gegeben.

Natriumrückstände wurden in Ethylalkohol aufgelöst und danach dem KOH-Reinigungsbad zugeführt. Auch die beim Vortrocknen der Lösungsmittel angefallenen festen $\mathrm{KOH}$ Rückstände wurden dem KOH-Reinigungsbad zugeführt.

Trockenmittelreste wie $\mathrm{P}_{4} \mathrm{O}_{10}$ wurden nach vorsichtiger Hydrolyse mit Wasser in die Basen- bzw. Säureabfälle überführt.

Konzentrierte Schwefelsäure/ $\mathrm{H}_{2} \mathrm{O}_{2}$-Lösung zur Reinigung von Glasfiltern wurde neutralisiert und in Schwermetallabfallbehältern gesammelt.

Schwermetallhaltige Abfälle wurden in den dafür vorgesehenen Behälter entsorgt. Alle anderen Chemikalienrückstände wurden vorsichtig mit wässrigem Aceton hydrolysiert und in die Gefäße für organische Abfälle gegeben.

Die bei dieser Arbeit angefallenen Mengen an Sonderabfällen können wie folgt abgeschätzt werden:

Halogenfreie Lösungsmittel: 15 L

Saure Chemikalienabfälle: $10 \mathrm{~L}$

Basische Chemikalienabfälle: $10 \mathrm{~L}$

Schwermetallhaltige Chemikalienabfälle: 2 L 


\section{Literaturverzeichnis}

[1] H. Bortels, Zentralbl. Bakteriol. Parasitenkd. Infektionskrankh. 95, 193 (1936).

[2] R. R. EAdy and B. E. Smith, A Treatise on Nitrogen Fixation, 399 (1979).

[3] J. Kim, D. Woo, and D. C. Rees, Biochemistry, 7104 (1993).

[4] R. Hille, Portland Press 34 (1999).

[5] J. B. Howard and D. C. Rees, Chem. Rev. 96, 2965 (1996).

[6] J. A. Patemann, D. J. Cove, B. M. Rever, and D. B. Roberts, Nature (London) 201, 58 (1964).

[7] P. A. Ketchum, H. Y. Cambrier, W. A. Frazier, C. H. Madansky, and A. Nason, Proc. Natl. Acad. Sci. USA 66, 1016 (1970).

[8] A. Nason, K. Y. Lee, S. S. Pan, P. A. Ketchum, A. Lamberti, and J. Devries, Proc. Natl. Acad. Sci. USA 68, 3242 (1971).

[9] J. L. Johnson, B. E. Hainline, and K. V. Rajagopalan, J. Biol. Chem. 255, $1783(1980)$.

[10] J. L. Johnson, B. E. Hainline, K. V. Rajagopalan, and B. H. Arison, J. Biol. Chem. 259, 5414 (1984). 
[11] E. C. TAylor, P. S. Ray, I. S. Darwish, J. L. Johnson, and K. V. Rajagopalan, J. Am. Chem. Soc. 111, 7664 (1989).

[12] M. J. Romao, M. Archer, I. Moura, J. J. G. Moura, J. LeGall, R. Engh, M. Schneider, P. Hof, and R. Huber, Science 270, 1170 (1995).

[13] H. Schindelin, C. Kisker, J. Hilton, K. V. Rajagopalan, and D. C. REES, Science 272, 1615 (1996).

[14] F. Schneider, J. Löwe, R. Huber, H. Schindelin, C. Kisker, and J. Knäblein, J. Mol. Biol. 263, 53 (1996).

[15] S. Bailey, A. S. McAlpine, E. M. H. Duke, N. Benson, and A. G. McEWAN, Acra Crystallogr., Sect. D. 52, 194 (1996).

[16] V. C. Boyington, V. N. Gladyshev, S. V. Khangulov, T. C. Stadtman, and P. D. Sun, Science 275, 1305 (1997).

[17] D. C. Rees, Y. Hu, C. Kisker, and H. Schindelin, Dalton Trans., 3909 (1997).

[18] M. G. Bertero, M. P. R. A. Rothery, C. Hou, D. Lim, F. Blasto, J. H. Weiner, and N. C. J. StrydnadkA, Nat. Struct. Biol. 10, 681 (2003).

[19] A. S. McAlpine, A. G. McEwan, A. L. Shaw, and S. Bailey, Inorg. Chem. 2, 690 (1997).

[20] M. K. Chan, S. Mukund, A. Kletzin, M. W. W. Adams, and D. C. Rees, Science 267, 1463 (1995).

[21] R. Huber, P. Hof, O. R. Duarte, J. J. G. Moura, I. Moura, M. Liu, J. LeGall, R. Hille, M. Archer, and M. J. Romao, Proc. Natl. Acad. Sci. USA 93, 8846 (1996). 
[22] M. R. Jones, F. E. Inscore, R. Hille, and M. L. Kirk, Inorg. Chem. 38, 4963 (1999).

[23] F. E. Inscore, R. McNaughton, B. L. Westscott, M. E. Helton, R. Jones, I. K. Dhawan, J. H. Enemark, and M. L. Kirk, Inorg. Chem. 38, 1401 (1999).

[24] B. L. Westscott, N. E. Gruhn, and J. H. Enemark, J. Am. Chem. Soc. 120, 3382 (1998).

[25] A. Kletzin and M. W. W. Adams, FEMS Microbiol. Rev. 18, 5 (1996).

[26] M. K. Johnson, D. C. Rees, and M. W. W. Adams, Chem. Revs. 96, 2817 (1996).

[27] L. G. LuUngdahl, Trends Biochem. Sci. 1, 63 (1976).

[28] I.Yamamoto, T. Saiki, S.-M. Liu, and L. G. Ljungdahl, J. Biol. Chem. 258, 1826 (1983).

[29] R. A. Schmitz, S. P. Albrecht, and R. K. Thauer, Eur. J. Biochem. 209, 1013 (1992).

[30] P. A. Bertram, R. A. Schmitz, D. Linder, and R. K. Thauer, Arch. Microbiol. 161, 220 (1994).

[31] P. A. Bertram, M. Karrasch, R. A. Schmitz, R. Bocher, S. P. AlBrecht, and R. K. Thauer, Eur. J. Biochem. 220, 477 (1994).

[32] A. Hochheimer, R. Hedderich, and R. K. Thauer, Arch. Microbiol. 170, 389 (1998).

[33] A. Sigel and H. Sigel, Metal Ions in Biological Systems 39, 187 (2002). 
[34] R. H. Holm, Chem. Rev. 87, 1401 (1987).

[35] R. Hille, Chem. Rev. 96, 2757 (1996).

[36] R. Hille, Trends Biochem. Sci. 27, 360 (2002).

[37] R. HilLe, Met. Ions Biol. Syst. 39, 187 (2002).

[38] M. J. Romao, M. Archer, I. Moura, J. J. G. Moura, J. LeGall, P. H. R. Engh, M. Schneider, and R. Huber, Science 270, 1170 (1995).

[39] H. Dobbek, L. Gremer, O. Meyer, and R. Huber, Proc. Natl. Acad. Sci. USA 96, 8884 (1999).

[40] R. M. Garrett and K. V. Rajagopalan, J. Biol. Chem. 271, 7387 (1996).

[41] C. C. Page, C. C. Moser, X. Chen, and P. L. Dutton, Nature 402, 47 (1999).

[42] Y. Kubo, N. Ogura, and H. Nakagawa, J. Biol. Chem. 263, 1968 (1988).

[43] G. N. George, J. A. Mertens, and W. H. Campbell, J. Am. Chem. Soc. 121, 9730 (1999).

[44] J. M. Dias, M. E. Than, A. Humm, R. Huber, G. P. Bourenkov, H. D. Bartunik, S. Bursakov, J. Calvete, J. Caldiera, C. Carniero, J. J. G. Moura, I. Moura, and M. J. RomaO, Structure Fold Res. 7, 65 (1999).

[45] J. C. Boyington, V. N. Gladyshev, S. V. Khangulov, T. C. Stadtman, and P. D. Sun, Science 275, 1305 (1997).

[46] P. Ellis, T. Conrads, R. Hille, and P. Kuhn, Structure 9, 125 (2001).

[47] M. K. Johnson, D. C. Rees, and M. W. W. Adams, Chem. Rev. 96, 2817 (1996). 
[48] B. G. Seiffert, G. M. Ullmann, A. Messerschmidt, B. Schink, P. M. H. Kroneck, and O. Einsle, Proc. Natl. Acad. Sci. USA 104, 3073 (2007).

[49] M. K. Chan, S. Mukund, A. Kletzin, M. W. Adams, and D. C. Rees, Science 267, 1463 (1995).

[50] R. H. Holm, Chem. Rev. 87, 1401 (1987).

[51] E. S. Davies, G. M. Aston, R. L. Beddoes, D. Collison, A. Dinsmore, A. Docrat, J. A. Joule, C. R. Wilson, and C. D. Garner, Dalton Trans. , 3647 (1998).

[52] B. S. Lim, K.-M. Sung, and R. H. Holm, J. Am. Chem. Soc. 122, 7410 (2000).

[53] K.-M. Sung and R. H. Holm, Inorg. Chem. 40, 4518 (2001).

[54] N. Ueyama, H. Oku, and A. Nakamura, J. Am. Chem. Soc. 114, 7310 (1992).

[55] S. K. Das, D. Biswas, R. Maiti, and S. Sarkar, J. Am. Chem. Soc. 118, 1387 (1996).

[56] G. C. Tucci, J. P. Donahue, and R. H. Holm, Inorg. Chem. 37, 1602 (1998).

[57] K. V. Rajagopalan, Adv. Enzymol. Relat. Areas Mol. Biol. 64, 215 (1991).

[58] K. V. Rajagopalan and J. L. Johnson, J. Biol. Chem. 267, 10199 (1992).

[59] C. Kisker, H. Schindelin, A. Pachero, W. A. Wehbi, J. H. E. R. M. Garrett, K.V. Rajagopalan, and D. C. Rees, Cell 91, 973 (1997). 
[60] G. N. George, I. J. Pickering, and C. Kisker, Inorg. Chem. 38, 2539 (1999).

[61] J. M. Berg and R. H. Holm, J. Am. Chem. Soc. 197, 925 (1985).

[62] J. M. Berg and R. H. Holm, J. Am. Chem. Soc. 107, 917 (1985).

[63] S. F. Geller, B. E. Schultz, M. J. Scott, and R. H. Holm, J. Am. Chem. Soc. 114, 6934 (1992).

[64] B. E. Schultz, S. F. Geller, M. C. Muetterties, M. J. Scott, and H. R. Holm, J. Am. Chem. Soc. 115, 2714 (1993).

[65] M. A. Pietsch and M. B. Hall, Inorg. Chem. 35, 1273 (1996).

[66] L. M. Thomson and M. B. Hall, Inorg. Chem. 123, 3995 (2001).

[67] Z. Xiao, C. G. Young, J. H. Enemark, and A. G. Wedd, J. Am. Chem. Soc. 114, 9191 (1992).

[68] Z. Xiao, M. A. Bruck, J. H. Enemark, C. G. Young, and A. Wedd, Inorg. Chem. 35, 7508 (1996).

[69] J. L. Johnson, B. E. Hainline, and K. V. Rajagopalan, J. Biol. Chem. 259, 5414 (1984).

[70] E. C. Taylor, P. S. Ray, I. S. Darwish, J. L. Johnson, and K. V. Rajagopalan, J. Am. Chem. Soc. 111, 7664 (1989).

[71] R. Eisenberg, Prog. Inorg. Chem. 12, 295 (1970).

[72] J. A. McCleverty, Prog. Inorg. Chem. 10, 49 (1968).

[73] U. T. Müller-Westerhoff and B. VAnce, Comprehensive Coordination Chemistry 2, 595 (1987). 
[74] G. N. Schrauzer and V. P. Mayweg, J. Am. Chem. Soc. 84, 3221 (1962).

[75] P. Cassoux and L. Valade, Inorganic Materials 2nd ed., 1996.

[76] N. Svenstrup and J. Becher, Synthesis, 215 (1995).

[77] R. Eisenberg and J. A. Ibers, Inorg. Chem. 4, 605 (1965).

[78] R. Eisenberg, J. A. Ibers, R. J. H. Clark, and H. B. Gray, J. Am. Chem. Soc. 86, 113 (1964).

[79] E. I. Stiefel, Progress in Inorganic Chemistry 52, 59 (2004).

[80] Autorenkollektiv, Organikum, VeB Deutscher Verlag der Wissenschaften, Berlin 1993.

[81] D. M. Giolando and K. Kirschbaum, Synthesis , 451 (1992).

[82] G. D. Figuly, C. K. Loop, and J. C. Martin, J. Am. Chem. Soc. 111, 654 (1989).

[83] N. D. Lowe and C. D. Garner, Dalton Trans., 2197 (1993).

[84] C. Lorber, J. P. Donahue, C. A. Goddard, E. Nordlander, and R. H. Holm, J. Am. Chem. Soc. 120, 8102 (1998).

[85] A. Maercker, Angew. Chem. 99, 1002 (1987).

[86] E. S. Davies, R. L. Beddoes, D. Collison, A. Dinsmore, A. Docrat, J. A. Joule, C. R. Wilson, and C. D. Garner, Dalton Trans., 3985 (1997).

[87] Y. Gareau, J. Chem. Soc., Chem. Commun., 1429 (1995).

[88] C. L. Socricelli, V. A. Szalai, and S. J. N. Burgmayer, J. Am. Chem. Soc. 113, 9877 (1991). 
[89] R. S. Pilato, K. A. Eriksen, M. A. Greaney, E. I. Stiefel, S. Goswami, L. Kilpatrick, T. G. Spiro, E. C. Taylor, and A. L. Rheingold, J. Am. Chem. Soc. 113, 9372 (1991).

[90] S. P. Kaiwar, J. K. Hsu, L. M. Liable-Sands, A. L. Rheingold, and R. S. Pilato, Inorg. Chem. 36, 4234 (1997).

[91] M. Peruzzini, I. Der Los Rios, and A. Romerosa, Prog. Inorg. Chem. 49, 169 (2001).

[92] S. Kuwata and M. Hidai, Coord. Chem. Rev. 213, 211 (2001).

[93] S. P. Kaiwar, A. Vodacek, N. V. Blough, and R. S. Pilato, J. Am. Chem. Soc. 119, 3311 (1997).

[94] G. P. Tucci, J. P. Donahue, and R. H. Holm, Inorg. Chem. 37, 1602 (1998).

[95] C. Lorber, J. P. Donahue, C. A. Goddard, E. Nordlander, and R. H. Holm, J. Am. Chem. Soc. 120, 8102 (1998).

[96] J. P. Donahue, C. R. Goldsmith, U. Nadiminti, and R. H. Holm, J. Am. Chem. Soc. 120, 12896 (1998).

[97] A. Thapper, J. P. Donahue, K. B. Musgrave, M. W. Willer, K. O. H. E. Nordlander, B. Hedman, and R. H. Holm, Inorg. Chem. 38, 4104 (1999).

[98] K. B. Musgrave, J. P. Donahue, C. Lorber, R. H. Holm, B. Hedman, and K. O. Hodgson, J. Am. Chem. Soc. 121, 10297 (1999).

[99] B. S. Lim, J. P. Donahue, and R. H. Holm, Inorg. Chem. 39, 263 (2000). 
[100] K.-M. Sung and R. H. Holm, Inorg. Chem. 39, 1275 (2000).

[101] K. B. Musgrave, B. S. Lim, K.-M. Sung, R. H. Holm, B. Hedman, and K. O. Hodgson, Inorg. Chem. 39, 5238 (2000).

[102] B. S. Lim, K.-M. Sung, and R. H. Holm, J. Am. Chem. Soc. 122, 7410 (2000).

[103] M. Miao, M. W. Willer, and R. H. Holm, Inorg. Chem. 39, 2843 (2000).

[104] B. S. Lim and R. H. Holm, J. Am. Chem. Soc. 123, 1920 (2001).

[105] K.-M. Sung and R. H. Holm, J. Am. Chem. Soc. 123, 1931 (2001).

[106] B. S. Lim and R. H. Holm, Inorg. Chem. 40, 645 (2001).

[107] R. Soyka, W. Pfleiderer, and R. Prewo, Helv. Chim. Acta 73, 808 (1990).

[108] R. Soyka and W. Pfleiderer, Pteridines 2, 63 (1990).

[109] B. Fischer and S. J. N. Burgmayer, Metal Ions in Biological Systems 39, $265(2002)$.

[110] B. Bradshaw, A. Dinsmore, C. D. Garner, and J. A. Joule, Chem. Comm. , 417 (1998).

[111] H. Sugimoto, M. Harihara, M. Shiro, K. Sugimoto, K. Tanaka, H. Miyake, and H. Tsukube, Inorg. Chem. 44, 6386 (2005).

[112] C. Schulzke, Dalton Trans., 713 (2005).

[113] R. H. Holm, Chem. Rev. 87, 1401 (1987).

[114] J. Jekö, T. Timar, and J. C. Jaszberenyi, J. Org. Chem. 56, 6748 (1991).

[115] H. K. Joshi and J. H. Enemark, J. Am. Chem. Soc. 126, 11784 (2004). 
[116] P. J. Ellis, T. Conrads, R. Hille, and P. Kuhn, Structure 9, 125 (2001).

[117] B. Ma, J.-H. LiI, H. F. S. III, and N. L. Allinger, J. Phys. Chem. 100, 8763 (1996).

[118] K. P. C. Vollhardt, Organische Chemie, VCH Verlagsgesellschaft mbH D-6940 Weinheim, 1990.

[119] G. Frenking and N. Fröhlich, Chem. Rev. 100, 717 u. Referenzen darin (2000).

[120] K. B. Wiberg, Tetrahedron 24, 1083 (1968).

[121] K. Ray, T. Petrenko, K. Wieghardt, and F. Neese, Dalton Trans. , 1552 (2007).

[122] B. S.Lim, D. V. Formitchev, and R. H. Holm, Inorg. Chem. 40, 4257 (2001).

[123] M. Fourmigue, Coord. Chem. Rev. 823, 178 (1998).

[124] S. I. Gorelsky, L. Basumallick, J. Vura-Weis, R. Sarangi, K. O. Hodgson, B. Hedman, K. Fujisawa, and E. I. Solomon, Inorg. Chem. 44, 4947 (2005).

[125] F. E. Inscore, R. McNaughton, B. L. Westscott, M. E. Helton, R. Jones, I. K. Dhawan, J. H. Enemark, and M. L. Kirk, Inorg. Chem. 38, 1401 (1999).

[126] R. L. McNaughton, M. E. Helton, N. D. Rubie, and M. L. Kirk, Inorg. Chem. 39, 4386 (2000).

[127] E. W. Garbisch and JR., J. Org. Chem 30, 2109 (1965). 
[128] K. Tanemura, T. Suzuki, K. Satsumabayashi, and T. Horaguchi, Chem. Commun., 470 (2004).

[129] H. J. KABBE, Synthesis, 886 (1978).

[130] P. Giori, T. Poli, A. C. Veronese, C. B. Vicentini, M. Manfrini, and M. Guarneri, J. Heterocyclic Chem. 23, 1661 (1986).

[131] M. J. Romao, M. Archer, I. Moura, J. J. Moura, J. LeGall, R. Engh, M. Schneider, P. Hof, and R. Huber, Science 270, 1170 (1995).

[132] J. P. McNamara, I. H. Hiller, T. S. Bhachu, and C. D. Garner, Dalton Trans., 3572 (2005).

[133] M. Kaupp, Angew. Chem. 116, 554 (2004).

[134] C. E. Webster and M. B. Hall, J. Am. Chem. Soc. 123, 5820 (2001).

[135] R. Huber, P. Hof, R. O. Duarter, J. J. G. Moura, I. Moura, M.-Y. Liu, J. Legall, R. Hille, M. Archer, and M. Romao, Proc. Natl. Acad. Sci. U.S.A. 93, 8846 (1996).

[136] A. D. Becke, J. Chem. Phys. 98, 5648 (1993).

[137] C. Lee, W. Yang, and R. G. Parr, Phys. Rev. B 37, 785 (1988).

[138] P. J. HaY and W. R. Wadt, J. Chem. Phys. 82, 270 (1985).

[139] F. Weinhold and C. R. Landis, Valency and Bonding: A Natural Bond Orbital Donor-Acceptor Perspective, Cambridge University Press, 2005.

[140] J. P. Foster and F. Weinhold, J. Am. Chem. Soc. 102, 7211 (1980).

[141] A. E. Reed and F. Weinhold, J. Chem. Phys. 83, 1736 (1985). 
[142] A. E. Reed, L. A. Curtiss, , and F. Weinhold, Chem. Rev. 88, 899 (1988).

[143] P.-O. Loewdin, Phys. Rev. 97, 1474 (1955).

[144] E. R. DAVIDSon, Reduced Density Matrices in Quantum Chemistry, Academic Press, New York, 1976.

[145] I. Meyer, Theor. Chim. Acta 67 67, 315 (1985).

[146] E. D. Glendening and F. Weinhold, J. Comput. Chem. 19, 610 (1998).

[147] C. J. Cramer, Essentials of Computational Chemistry: Theories and Models, 2nd Ed., Wiley, 2004.

[148] M. J. Frisch, G. W. Trucks, H. B. Schlegel, G. E. Scuseria, M. A. Robb, J. R. Cheeseman, J. A. Montgomery, Jr., T. Vreven, K. N. Kudin, J. C. Burant, J. M. Millam, S. S. Iyengar, J. Tomasi, V. Barone, B. Mennucci, M. Cossi, G. Scalmani, N. Rega, G. A. Petersson, H. Nakatsuji, M. Hada, M. Ehara, K. Toyota, R. Fukuda, J. Hasegawa, M. Ishida, T. Nakajima, Y. Honda, O. Kitao, H. NaKai, M. Klene, X. Li, J. E. Knox, H. P. Hratchian, J. B. Cross, V. Bakken, C. Adamo, J. Jaramillo, R. Gomperts, R. E. Stratmann, O. Yazyev, A. J. Austin, R. Cammi, C. Pomelli, J. W. Ochterski, P. Y. Ayala, K. Morokuma, G. A. Voth, P. Salvador, J. J. Dannenberg, V. G. Zakrzewski, S. DapPrich, A. D. Daniels, M. C. Strain, O. Farkas, D. K. Malick, A. D. Rabuck, K. Raghavachari, J. B. Foresman, J. V. Ortiz, Q. Cui, A. G. Baboul, S. Clifford, J. Cioslowski, B. B. Stefanov, G. Liu, A. Liashenko, P. Piskorz, I. Komaromi, R. L. Martin, D. J. Fox, T. Keith, M. A. Al-Laham, C. Y. Peng, A. Nanayakkara, M. Challacombe, P. M. W. Gill, B. Johnson, W. Chen, M. W. Wong, C. Gonzalez, 
and J. A. Pople, Gaussian 03, Revision C.02, Gaussian, Inc., Wallingford, CT, 2004 .

[149] R. Ahlrichs, M. BÄr, M. HÄser, H. Horn, and C. Kölmel, Chem. Phys. Lett. 162, 165 (1989).

[150] G. SchaftenaAR, Molden Version 3.2, CAOS/CAMM Center, University of Nifmegen, The Nederlands.

[151] Flükiger, H. LÜthi, S. Portmann, and J. Weber, Swiss National Supercomputing Centre CSCS, Manno (Switzerland), 2000.

[152] A. B. Smith, III, R. A. Rivero, K. J. Hale, and H. A. Vaccaro, J. Am. Chem. Soc. 113, 2092 (1991).

[153] J. P. Smit, W. Purcell, A. Roodt, and J. G. Leopldt, Polyhedron 12, 2271 (1993).

[154] A. Roodt, S. S. Bassoon, and J. G. Leopoldt, Polyhedron 13, 599 (1994). 


\section{Veröffentlichungen}

(Stand September 2007)

[1] Z. Yang, X. Ma, R. B. Oswald, H. W. Roesky, H. Zhu, C.

Schulzke, K. Starke, M. Baldus, H.-G. Schmidt and M.

Noltemeyer, Angew. Chem. 44, 7072 (2005).

[2] L. W. Pineda, V. Jancik, K. Starke, R. B. Oswald and H. W. Roesky, Angew. Chem. 45, 2602 (2006).

[3] H. Zhu, R. B. Oswald, H. Fan, H. W. Roesky, Q. Ma, Z. Yang, H.-G. Schmidt, M. Noltemeyer, K. Starke and N. S. Hosmane, J. Am. Chem. Soc. 128, 5100 (2006).

[4] X. Ma, K. Starke, C. Schulzke, H.-G. Schmidt and M. Noltemeyer, Eur. J. Inorg. Chem., 628 (2006).

[5] Q. Zhang, K. Starke, C. Schulzke, A. Hofmeister and J. Magull, Inorganica Chimica Acta 360, 3400 (2007). 